\title{
Bott's Periodicity Theorem from the Algebraic Topology Viewpoint
}

\author{
Luciana Basualdo Bonatto
}

Dissertation SubmitTed

TO THE

Institute of MATHEMATICS AND Statistics

OF THE

University of SÃo PAUlo

FOR

THE DEGREE

OF

MASTER OF SCIENCE

Program: Mathematics

Supervisor: Prof. Dr. Ivan Struchiner

During the development of this dissertation the author was financially supported by the CNPq 


\section{Bott's Periodicity Theorem from the Algebraic Topology Viewpoint}

This is the original version of the dissertation developed by the candidate Luciana Basualdo Bonatto, as it was submitted to the Judging Committee.

Judging Committee:

- Prof. Dr. Ivan Struchiner - IME-USP

- Prof. Dr. Mariana Rodrigues da Silveira - UFABC

- Prof. Dr. Carlos Henrique Grossi Ferreira - ICMC-USP 
To my dearest friend Patrícia. 


\section{Acknowledgement}

I would first like to thank my supervisor Professor Dr. Ivan Struchiner for all the support and friendship during the process of developing this dissertation. I would also like to thank my former supervisors Professor Dr. Hugo Luiz Mariano and, one of my heroines, Professor Dr. Zara Issa Abud. I will always be grateful for your kindness, support and for being able to share with all of you the love for Mathematics.

I would also like to thank my family and all my dear friends, who kept me standing during this journey and who always help me rediscover love and beauty in life.

Finally, I would like to thank the members of the Judging Committee for the consideration and helpful comments, and the CNPq for the support which made it possible for me to develop this dissertation. 


\begin{abstract}
In 1970, Raoul Bott published The Periodicity Theorem for the Classical Groups and Some of Its Applications, in which he uses this famous result as a guideline to present some important areas and tools of Algebraic Topology. This dissertation aims to use the path Bott presented in his article as a guideline to address certain topics on Algebraic Topology.

We start this incursion developing important tools used in Homotopy Theory such as spectral sequences and Eilenberg-MacLane spaces, exploring how they can be combined to aid in computation of homotopy groups. We then study important results of Morse Theory, a tool which was in the centre of Bott's proof of the Periodicity Theorem. We also develop two extensions: Morse-Bott Theory, and the applications of such results to the loopspace of a manifold. We end by giving an introduction to generalised cohomology theories and K-Theory.
\end{abstract}

Keywords: Bott's Periodicity Theorem, Homotopy Theory, Spectral Sequences, Eilenberg-MacLane spaces, Morse Theory, Morse-Bott Theory, Generalised Cohomology, K-Theory. 



\section{Resumo}

Em 1970, Raoul Bott publicou o artigo The Periodicity Theorem for the Classical Groups and Some of Its Applications no qual usava esse famoso resultado como um guia para apresentar importantes áreas e ferramentas da Topologia Algébrica. O presente trabalho usa o mesmo caminho traçado por Bott em seu artigo como roteiro para explorar tópicos importantes da Topologia Algébrica.

Começamos esta incursão desenvolvendo ferramentas importantes da Teoria de Homotopia como sequências espectrais e espaços de Eilenberg-MacLane, explorando como estes podem ser combinados para auxiliar em cálculos de grupos de homotopia. Passamos então a estudar resultados importantes de Teoria de Morse, uma ferramenta que estava no centro da demonstração de Bott do Teorema da Periodicidade. Desenvolvemos ainda, duas extensões: Teoria de Morse-Bott e aplicações destes resultados ao espaço de laços de uma variedade. Terminamos com uma introdução a teorias de cohomologia generalizadas e K-Teoria.

Palavras-chave: Teorema da Periodicidade de Bott, Teoria de Homotopia, Sequências Espectrais, Espaços de Eilenberg-MacLane, Teoria de Morse, Teoria de Morse-Bott, Cohomologia Generalizada, K-Teoria. 



\section{Contents}

\begin{tabular}{ll}
\hline Introduction & 1
\end{tabular}

1 Tools and Computations of Homotopy Groups 5

1.1 Computation of Stable Homotopy of the Classical Groups . . . . . . . . . . 6

1.2 Spectral Sequences $\ldots \ldots \ldots \ldots \ldots \ldots$

$1.2 .1 \quad$ Spectral sequence of a Filtered Complex . . . . . . . . . . . . . . . . 10

1.2 .2 Spectral Sequence of a Fibration . . . . . . . . . . . . . . . . . 20

1.3 Eilenberg-MacLane Spaces . . . . . . . . . . . . . . . . . . . . . . . . . . . . 32

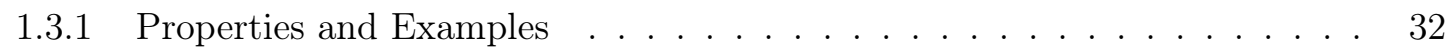

1.3 .2 Postnikov Towers . . . . . . . . . . . . . . . . . . . . . . . . . 40

2 Bott's Approach to the Periodicity Theorem 46

2.1 Morse Theory . . . . . . . . . . . . . . . . . . . . . . . . . 47

$2.2 \quad$ Morse-Bott Theory . . . . . . . . . . . . . . . . . . . . . . . . . . 65

2.3 Morse Theory on the Loop Space $\ldots \ldots \ldots$. . . . . . . . . . . . . . 71

2.4 The Periodicity Theorem for the Classical Groups . . . . . . . . . . . . . . . . 88

\begin{tabular}{|ll}
\hline 3 An Introduction to K-Theory & 91
\end{tabular}

3.1 Cohomology Theories . . . . . . . . . . . . . . . . . . . . . . . . 9 91

3.2 The Geometric Approach to K-Theory . . . . . . . . . . . . . . . . . . . . . . . . 109

\begin{tabular}{lr}
\hline Bibliography & 116
\end{tabular} 


\section{Introduction}

Algebraic Topology aims to answer topological questions by means of transforming them into well-posed algebraic problems. This can be done by using functors from the category of topological spaces and continuous maps with values on groups, rings, modules and many other algebraic structures. There are many functors which make such connections and some of the most wellknown are the ones defined by homology or cohomology theories. In this work, we will focus on one of these bridges between topology and algebra: Homotopy Theory.

Homotopy Theory is one of the many important tools and theories which compose Algebraic Topology. It is based on a very intuitive idea of identifying geometric objects that can be continuously deformed into each other. The origins of homotopy concept for paths can be traced back to calculus of variations, in which it was used as a visual tool to decide whether two paths lead to the same result for integration. The fundamental group was the first of the homotopy functors to be properly defined by Poincaré in 1895 , as the set $\left[S^{1}, s_{0} ; X, x_{0}\right]$ of homotopy classes from maps $\left(S^{1}, s_{0}\right) \rightarrow\left(X, x_{0}\right)$, equipped with an intuitive group structure and denoted $\pi_{1}\left(X, x_{0}\right)$. Intuitively, we can think of it measuring the "1-dimensional" holes on the space $X$ on the arcconnected component of $x_{0}$. It was then natural to consider the high-dimensional analogues, looking now at the classes $\left[S^{n}, s_{0} ; X, x_{0}\right]$ which could also be equipped with a group structure, and originated the higher homotopy groups $\pi_{n}\left(X, x_{0}\right)$.

One of the beautiful things about homotopy groups is that their definition is extremely intuitive, in opposition, for instance, to homology and cohomology groups. And, since this intuition made them seem too simple, for many years high homotopy groups did not generate much interest. This changed in 1931, when Hopf showed that $\pi_{3}\left(S^{2}\right)$ was nontrivial, precisely, by defining an equivalence relation in $S^{3}$, the unit sphere in $\mathbb{C}^{2}$, by

$$
\left(z_{0}, z_{1}\right) \sim\left(w_{0}, w_{1}\right) \text { if and only if }\left(z_{0}, z_{1}\right)=\left(\lambda w_{0}, \lambda w_{1}\right)
$$

for some complex number $\lambda$ of absolute value 1 . The quotient of this equivalence relation $S^{3} / \sim$ is, by definition, the complex projective space $C P^{1}$, which is actually homeomorphic to $S^{2}$. This map defined what was called the Hopf fibration. Hopf has shown that such map $S^{3} \rightarrow S^{2}$ is not homotopic to the constant map on $S^{2}$ and that implies that the third homotopy group of $S^{2}$ is not trivial.

This result came as a great surprise since it was expected that such an intuitive object, one that carried the visual interpretation of counting holes, would have a simple structure when evaluated on basic objects such as spheres, as was the case for homology and cohomology groups. This 
led to new questions on high homotopy groups and how to compute them, which turned outto be an extremely difficult task. For instance, determining the groups $\pi_{m}\left(S^{n}\right)$ for $m>n$ is still a question not completely answered.

Many new techniques were then developed to aid in such investigations, as for instance, the definition of Eilenberg-MacLane spaces. These are constructed to have well-behaved homotopy groups and to be used as the homotopic analogues for spheres in homology theory. It was then interesting to see that, parallel to the way CW-complexes can be built through spheres, a $\mathrm{CW}$ complex can also be "constructed" from Eilenberg-MacLane spaces using fibrations, and that this structure helps one determine its homotopy groups.

One of the strategies which was being used on such computations was to look for relations between homotopy and the (much easier to calculate) homology groups, among which the wellknown Hurewicz Theorem stands out. Therefore, when studying homotopy, many tools related to such other functor become very important, among which we highlight Serre's Spectral Sequence, which relates homology groups of a fibration.

The combination of Eilenberg-MacLane spaces to such Spectral Sequences yield very important results on high homotopy groups. For instance, in Singular Homology of Fiber Spaces ([Ser12]), Serre was able to show that if $i>n$, then the groups $\pi_{i}\left(S^{n}\right)$ are finite, except $\pi_{2 n-1}\left(S^{n}\right)$, for an even $n$, which is a direct sum of $\mathbb{Z}$ and a finite group. However, even with such equipment, this computation remained an extremely difficult task.

However, not only homotopy groups of spheres were investigated. For instance, we can see in Serre's article Homotopy Groups and Classes of Abelian Groups $\left(\left[\mathrm{SBM}^{+} 12\right]\right)$ that there was a lot of work being done to determine homotopy groups of compact Lie groups, and specifically of the simple Lie groups. These, which are classified into nine different types, can be thought of as atomic "blocks" that make up all (finite-dimensional) connected Lie groups. Four such groups are called classical and the others, exceptional. The classical simple Lie groups have very well known simply connected representatives: $S U(n), S O(2 n+1), S p(n), S O(2 n)$. It is in this context that the homotopy groups of such spaces were studied, and computing them was shown to be extremely difficult. After all, it wasn't simple even for the "basic spaces" of algebraic topology, the spheres, let alone to such spaces.

Therefore, it came as a great surprise when, in 1959, Raoul Bott demonstrated ([Bot59]) that the stable homotopy of the classical groups is periodic. The precise meaning of such a statement will be discussed next, but the wonder it provoked can be already comprehended.

These groups play important roles in Algebraic and Differential Topology and Geometry, and their topological properties were thoroughly discussed in the 20th century. For instance, every real (resp. complex) vector bundle of rank $m$ is associated with a principal fiber space with structural group $O(m)$ (resp. $U(m)$ ). One interesting property already known about the homotopy groups of such spaces is that they eventually become stable, that is, if $n$ is large enough, then the $q$-th homotopy groups of $O(n), U(n)$ and $S p(n)$ are independent of $n$. We are then able to 
define the stable homotopy groups $\pi_{q}(O), \pi_{q}(U)$ and $\pi_{q}(S p)$ of such spaces:

$$
\begin{aligned}
\pi_{q}(O) & =\lim _{i \rightarrow \infty} \pi_{q}(O(i)) \\
\pi_{q}(U) & =\lim _{i \rightarrow \infty} \pi_{q}(U(i)) \\
\pi_{q}(S P) & =\lim _{i \rightarrow \infty} \pi_{q}(S p(i))
\end{aligned}
$$

With this in hand, we are able to state the Periodicity Theorem for the Classical Groups:

Theorem 0.0.1 (Bott's Periodicity Theorem): The homotopy groups of $O, U$ and Sp satisfy the recursion relation:

$$
\begin{aligned}
\pi_{k}(U) & =\pi_{k+2}(U) \\
\pi_{k}(O) & =\pi_{k+4}(S p) \\
\pi_{k}(S p) & =\pi_{k+4}(O)
\end{aligned}
$$

This shows us that $\pi_{*}(U)$ has period two, and both $\pi_{*}(O)$ and $\pi_{*}(S p)$ have period eight, but also, since the first few homotopy groups of these spaces can be computed without much difficulty, this result gives us a complete account of the stable homotopy groups of such spaces.

It is very interesting that such an impressive and surprising result on homotopy groups was actually obtained using very different techniques than the ones which were used to compute them at that time. In the article The stable homotopy of the classical groups, Bott establishes such relations using an alternative famous definition for the homotopy groups: let $\Omega\left(X, x_{0}\right)$ be the set of maps $\left(S^{1}, s_{0}\right) \rightarrow\left(X, x_{0}\right)$ with the compact-open topology and basepoint $\omega_{0}$ the constant map equals to $x_{0}$, then it is simple to see that there is an equivalence:

$$
\pi_{n}\left(X, x_{0}\right) \simeq \pi_{n-1}\left(\Omega\left(X, x_{0}\right), \omega_{0}\right)
$$

Bott's idea was to then establish further relations between the homotopy groups of such spaces by constructing a CW-structure to $\Omega\left(X, x_{0}\right)$. This was carried out using an extension of Morse Theory, a branch from differential topology which provided tools to create a "cellular structure" on a manifold $M$ from a well-behaved function $M \rightarrow \mathbb{R}$. The Periodicity Theorems are then obtained by applying those techniques to the classical groups.

More than an impressive result, Bott's Periodicity Theorem became very important for the creation of K-Theory, a generalised cohomology theory which was at the centre of famous results such as on division algebras over the real numbers, a solution on the Hopf conjecture and the Atiyah-Singer index theorem, among many other important results. The importance of such a theory may be described by the fact that it has a very intuitive and important geometric interpretation looking at vector bundles, which endows it with many operations from linear algebra, and on the other hand, it can be seen to be a representable functor with periodic values (a result which follows directly from the periodicity theorems).

In 1970, Bott wrote The periodicity theorem for the classical groups and some of its applications 
([Bot70]), in which he uses the Periodicity Theorem as a background to explore interesting topics of algebraic and differential topology. In this dissertation, we do the same: we explore famous and important topics using the path which Bott created (and which is organised around the Periodicity Theorem) as a guide. We assume some knowledge on High Homotopy Groups, CW complexes, Fibrations, Homological Algebra and Riemmanian Geometry.

This exploration can be divided into three parts, which correspond to the chapters of this dissertation: the tools and theories which were used to study and compute homotopy; theories of algebraic and differential topology which were used on the original proof of Bott's Periodicity Theorem; and finally an introduction to K-Theory.

Chapter 1, Tools and Computations of Homotopy, will discuss Eilenberg-MacLane spaces and Serre's Spectral Sequence, exploring the ideas involved in their definitions and how such tools were combined to discover homotopy groups.

In Chapter 2, Bott's Approach to the Periodicity Theorem, we use tools from Differential Topology, Riemannian Geometry and Calculus of Variations, to explore Morse Theory and its extensions which were used in the original proof of Bott's Theorem.

In the final chapter, Chapter 3, An Introduction to K-Theory, we explore the matters of generalised cohomology through two different approaches to K-Theory: one via representable cohomologies, and the other through its geometric interpretation. We explore those natural definitions, how they are related to the Periodicity Theorem, and how they combine to yield this powerful and useful theory. 


\section{Chapter 1}

\section{Tools and Computations of Homotopy Groups}

This chapter will discuss some methods and computations of Homotopy Groups.

In Section 1.1 we recall the precise definitions of $O(n), U(n)$ and $S p(n)$, and show how Bott's Periodicity Theorem allows us to compute the stable homotopy of the Classical Groups using simple tools from Homotopy Theory.

The rest of this chapter will address two important tools used on computations of Homotopy Groups, which were singled out in Bott's article The Periodicity Theorem for The Classical Groups and Some of its Applications: Spectral Sequences and Eilenberg-MacLane spaces.

The main goal of Section 1.2 is to develop the Spectral Sequence for Fibrations and show how it has very useful properties which can be useful for computations of homology and homotopy groups. We give the general definition of this tool and then, while constructing the Spectral Sequence of a Filtration, give an intuitive idea of how it works and how it can be seen to approximate homology. We close with some famous computations which can be done with this tool. The main references used in this section are [Cho06], [Hat04], [McC01], [Ser12] and [BT82].

In Section 1.3 we give the definition of Eilenberg-MacLane spaces, and also provide examples and results on existence and uniqueness. We then detail how every CW-complex can be thought of as built from such spaces through the construction of Postnikov Towers. We end by showing how such tools can be combined with spectral sequences to compute homotopy groups. The main references used in this section are [DK01], Hat02], BT82] and Ser53]. 


\subsection{Computation of Stable Homotopy of the Classical Groups}

The Periodicity Theorem for the Classical Groups, which was proven by Bott in 1959, states that the stable homotopy groups of $O(n), U(n)$ and $S p(n)$ satisfy the recursion relation:

$$
\begin{aligned}
\pi_{k}(U) & =\pi_{k+2}(U) \\
\pi_{k}(O) & =\pi_{k+4}(S p) \\
\pi_{k}(S p) & =\pi_{k+4}(O)
\end{aligned}
$$

We start by recalling the definitions of the Lie groups which are studied and discussing in which sense their homotopy groups are stable.

$O(n)$ : The $n$-th Orthogonal Group consists of all linear maps $\mathbb{R}^{n} \rightarrow \mathbb{R}^{n}$ which preserve the inner product on $\mathbb{R}^{n}$. Alternatively, $O(n)$ is the group of all real $n \times n$ matrices $A$ such that $A A^{t}=I$.

$U(n)$ : The $n$-th Unitary Group consists by all linear transformations $\mathbb{C}^{n} \rightarrow \mathbb{C}^{n}$ which preserve the hermitian product on $\mathbb{C}^{n}$. Equivalently, $U(n)$ is the group of all $n \times n$ complex matrices $A$ such that $A A^{*}=I$, where $A^{*}$ is the conjugate transpose of $A$.

$S p(n)$ : The $n$-th Symplectic Group consists by all linear transformations $\mathbb{H}^{n} \rightarrow \mathbb{H}^{n}$ which preserve the hermitian form on $\mathbb{H}^{n}$. In other words, $S p(n)$ is the group of all $n \times n$ quaternionic matrices $A$ such that $A A^{*}=I$, where $A^{*}$ is the conjugate transpose of $A$.

We can see that there are natural inclusions $O(n) \hookrightarrow O(n+1), U(n) \hookrightarrow U(n+1)$ and $S p(n) \hookrightarrow$ $S p(n+1)$.

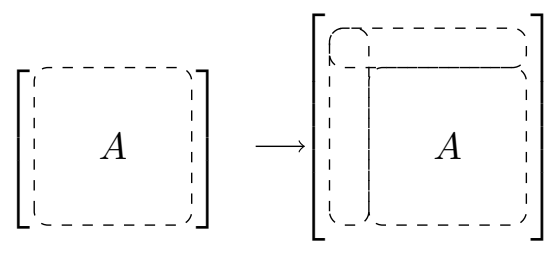

It is simple to show that such inclusions are in fact fibrations, which are described below:

$$
\begin{aligned}
& O(n) \rightarrow O(n+1) \quad \rightarrow \quad S^{n} \\
& U(n) \rightarrow U(n+1) \rightarrow S^{2 n+1} \\
& S p(n) \rightarrow S p(n+1) \rightarrow S^{4 n+3}
\end{aligned}
$$

These maps give us much information regarding the homotopy groups of such spaces, due to the following famous result of Algebraic Topology:

Theorem 1.1.1: (Long Exact Sequence of a Fibration) Given a fibration $F \hookrightarrow E \rightarrow B$ with $B$ path-connected, the following sequence is exact.

$$
\begin{aligned}
\cdots \rightarrow \pi_{n}(F) & \rightarrow \pi_{n}(E) \rightarrow \pi_{n}(B) \rightarrow \pi_{n-1}(F) \rightarrow \pi_{n-1}(E) \rightarrow \cdots \\
& \rightarrow \pi_{1}(E) \rightarrow \pi_{1}(B) \rightarrow \pi_{0}(F) \rightarrow \pi_{0}(E) \rightarrow \pi_{0}(B)
\end{aligned}
$$


Note that, in general, $\pi_{0}(F), \pi_{0}(E), \pi_{0}(B)$ are thought of simply as sets with no structure of groups.

A proof of such a result is seen in Hatcher's Algebraic Topology [Hat02] (Theorem 4.41).

Therefore, the fibrations above give us long exact sequences of homotopy groups. For instance, for $O(n)$, we get:

$$
\cdots \longrightarrow \pi_{q+1}\left(S^{n}\right) \longrightarrow \pi_{q}(O(n+1)) \longrightarrow \pi_{q}(O(n)) \longrightarrow \pi_{q}\left(S^{n}\right) \longrightarrow \cdots
$$

Since the first homotopy groups of spheres are known to be trivial, this shows us that $\pi_{q}(O(n+1))$ is isomorphic to $\pi_{q}(O(n))$, for all $q<n-1$.

This means that the homotopy groups of such spaces become stable, in the sense that, for all positive integer $q$, there will be an $n>0$, such that $\pi_{q}(O(n)) \simeq \pi_{q}(O(n+i))$ for all $i>0$. Such $\pi_{q}(O(n))=\lim _{i \rightarrow \infty} \pi_{q}(O(i))$ is called the $q$-th stable homotopy group of the orthogonal group, and is denoted as $\pi_{q}(O)$.

From the other fibrations shown above, we can see that the same property holds for the homotopy of both the unitary and symplectic groups. With this, we define, as we did above, their $q$-th stable homotopy groups, denoted as $\pi_{q}(U)$ and $\pi_{q}(S p)$ respectively. These groups are the ones to which Bott's Periodicity Theorem refers and actually, using this result, the task of determining the stable homotopy of $U(n), O(n)$ and $S p(n)$ becomes straightforward. The rest of this section will be dedicated to such computations.

We start by calculating the homotopy groups of $U$, through the exact sequence for the fibration $U(n) \rightarrow U(n+1) \rightarrow S^{2 n+1}:$

$$
\cdots \longrightarrow \pi_{q+1}\left(S^{2 n+1}\right) \longrightarrow \pi_{q}(U(n+1)) \longrightarrow \pi_{q}(U(n)) \longrightarrow \pi_{q}\left(S^{2 n+1}\right) \longrightarrow \cdots
$$

We see that the groups $\pi_{q}(U(n+1))$ and $\pi_{q}(U(n))$ are isomorphic, for all $q<2 n$. Therefore, to understand $\pi_{0}(U)$ and $\pi_{1}(U)$, which determine all homotopy groups of $U$, it is enough to study $\pi_{0}(U(1))$ and $\pi_{1}(U(1))$. Since $U(1)$ is actually $S^{1}$, we get:

$$
\begin{aligned}
\pi_{2 k}(U) & =\pi_{0}(U)=\pi_{0}\left(S^{1}\right)=0 \\
\pi_{2 k+1}(U) & =\pi_{1}(U)=\pi_{1}\left(S^{1}\right)=\mathbb{Z}
\end{aligned}
$$

Regarding $O$ and $S p$, we can also compute such groups by looking at fibrations on such spaces. For instance, from the homotopy exact sequence for $S p(n) \rightarrow S p(n+1) \rightarrow S^{4 n+3}$,

$$
\cdots \longrightarrow \pi_{q+1}\left(S^{4 n+3}\right) \longrightarrow \pi_{q}(S p(n+1)) \longrightarrow \pi_{q}(S p(n)) \longrightarrow \pi_{q}\left(S^{4 n+3}\right) \longrightarrow \cdots
$$

we see that the groups $\pi_{q}(S p(n+1))$ and $\pi_{q}(S p(n))$ are isomorphic, for all $q<4 n+2$. Therefore, the homotopy groups of $S p(1)$ already determine all stable homotopy groups of $S p$, except in dimensions six and seven. Since $S p(1)$ is actually $S^{3}$, we know that

$$
\pi_{k+8 i}(S p)=\pi_{k}(S p)=\pi_{k}\left(S^{3}\right)
$$


for all $k=0,1, \ldots, 5$. This show us that

$$
\pi_{8 i}(S p)=0, \quad \pi_{1+8 i}(S p)=0, \quad \pi_{2+8 i}(S p)=0, \quad \pi_{3+8 i}(S p)=\mathbb{Z}
$$

To determine $\pi_{6}(S p)=\pi_{2}(O)$ and $\pi_{7}(S p)=\pi_{3}(O)$ it is enough to observe that $S O(3)$ (one of the two homeomorphic connected components of $O(3))$, is the real projective space $\mathbb{R} P^{3}$. Then, from the double covering $S^{3} \rightarrow \mathbb{R} P^{3}$, we obtain a long exact sequence in homotopy

$$
\cdots \longrightarrow \pi_{q+1}\left(\mathbb{Z}_{2}\right) \longrightarrow \pi_{q}\left(S^{3}\right) \longrightarrow \pi_{q}\left(\mathbb{R} P^{3}\right) \longrightarrow \pi_{q}\left(\mathbb{Z}_{2}\right) \longrightarrow \cdots
$$

since $\pi_{q}\left(\mathbb{Z}_{2}\right)=0$, for all $q \geq 1$, we can see that

$$
0=\pi_{2}(S O(3))=\pi_{2}(O(3)) \quad \mathbb{Z} \simeq \pi_{3}(S O(3))=\pi_{3}(O(3))
$$

From the relation above, we also conclude that $\pi_{4}\left(S^{3}\right)=\pi_{0}(O(3)) \approx \mathbb{Z}_{2}$ and $\pi_{5}\left(S^{3}\right)=\pi_{1}(O(3))=$

\begin{tabular}{|c|c|c|c|c|c|c|c|c|c|c|c|}
\hline$i(\bmod 8)$ & 0 & 1 & 2 & 3 & 4 & 5 & 6 & 7 & $i(\bmod 2)$ & 0 & 1 \\
\hline$\pi_{i}(O)$ & $\mathbb{Z}_{2}$ & $\mathbb{Z}_{2}$ & 0 & $\mathbb{Z}$ & 0 & 0 & 0 & $\mathbb{Z}$ & $\pi_{i}(U)$ & 0 & $\mathbb{Z}$ \\
\hline$\pi_{i}(S p)$ & 0 & 0 & 0 & $\mathbb{Z}$ & $\mathbb{Z}_{2}$ & $\mathbb{Z}_{2}$ & 0 & $\mathbb{Z}$ & & & \\
\hline
\end{tabular}
$\pi_{1}\left(\mathbb{R} P^{3}\right) \approx \mathbb{Z}_{2}$.

Therefore, we have a general account of the stable homotopy of the Classical Groups:

Although this is a very important result about classical groups, there were still many open questions related to them, for instance, about how to determine the non-stable homotopy groups of such spaces. We will see in the next section some techniques used in such computations.

\subsection{Spectral Sequences}

In this section, we introduce Spectral Sequences, an apparatus which was described in Swi75] as one of the "topologist's most effective tools". It was first developed between 1940-45 by Jean Leray, a French applied mathematician, when he was a prisoner in a World War II concentration camp. Apparently Leray was afraid that the Nazis would exploit his applied knowledge to their benefit, and this motivated him to do research in algebraic topology, an area which he thought of as "safe" from such usage. His work resulted in the creation of spectral sequences.

Pictorially, we begin with some initial data (like a sequence of subspaces) and produce an infinite book whose pages are related and which, under favourable conditions, will eventually stabilise. This book is our spectral sequence and, under suitable conditions, we may use such stable pages to regain information on our original data. It may seem as a tricky procedure depending a lot on "good conditions" and indeed, in [DK01], Davis compares spectral sequences to techniques for computing integrals: "it is helpful to have ingenuity and a supply of tricks, and even so, you may not arrive at the final solution to your problem".

However complicated, this machinery has applications beyond algebraic topology. Even within this area, there are many constructions of spectral sequences which produce very important 
results. We will explore two well-known examples, the ones which can be obtained from a filtration, and the ones which can be obtained from a fibration. Specifically, we will work with homology spectral sequences, although most of the procedure can be easily dualized to yield a cohomology version.

In a simple manner of speaking, one can say that a spectral sequence is a sequence of differential groups $\left\{E^{r}, d^{r}\right\}$ with $d^{r} \circ d^{r}=0$ and such that $E^{r}$ is isomorphic to $\frac{\operatorname{ker} d^{r-1}}{\operatorname{Im} d^{r-1}}$, where the isomorphisms are fixed as part of the structure of the spectral sequence. This is a very powerful algebraic tool that, in Topology, is much used to calculate homology and cohomology groups. In fact, each $\left\{E^{r}, d^{r}\right\}$ can be thought of as a page of a book in which each $E^{r}$ is an approximation of the desired homology (resp. cohomology) group.

Since the concept of a spectral sequence is extremely abstract and vague, there are many types of spectral sequences and many ways to construct one, that is, ways to define each $E^{r}$ and $d^{r}$. Notice, for instance, that although $E^{r+1}$ is determined by $E^{r}$ and $d^{r}$, the differential $d^{r+1}$ is not, there is still a choice to be made.

A common method for constructing a spectral sequence is from an exact couple.

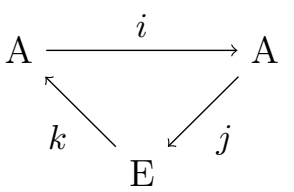

Definition 1.2.1: An exact couple is an exact sequence of abelian groups, such as the one on the left, where $i, j$ and $k$ are group homomorphisms.

Example 1.2.1: $\quad$ From a short exact sequence of differential complex like the one below

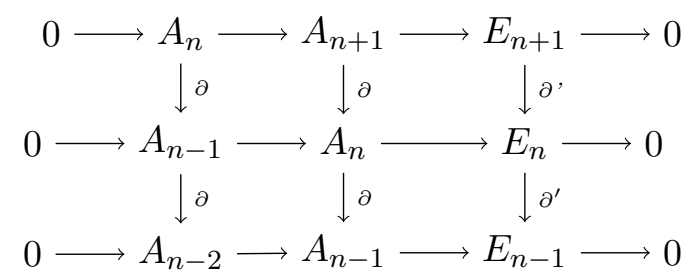

we get a long exact sequence of homology groups:

$$
\cdots \rightarrow H\left(E_{n+1}\right) \stackrel{k}{\rightarrow} H\left(A_{n-1}\right) \stackrel{i}{\rightarrow} H\left(A_{n}\right) \stackrel{j}{\rightarrow} H\left(E_{n}\right) \stackrel{k}{\rightarrow} H\left(A_{n-2}\right) \rightarrow \ldots
$$

Then, setting $A=\bigoplus_{n} H\left(A_{n}\right)$ and $E=\bigoplus_{n} H\left(E_{n}\right)$, and using as $i, j$ and $k$ the operators in Sequence 1.1, we get an exact couple.

The term $E$ in our exact couple, will be the group in one of the pages of our spectral sequence. The idea is that, from an exact couple, we can obtain a differential $d: E \rightarrow E$ and another exact couple with $E$-term as the homology group of $d$. Iterating this process we obtain a spectral sequence. 
Definition 1.2.2: From a given exact couple, we can construct what we call the derived couple by defining a differential operator. Let $d: E \rightarrow E$ be $d=j \circ k$, so the group $H(E)=\frac{K e r d}{I m d}$ is defined. Then the derived couple is a triple:

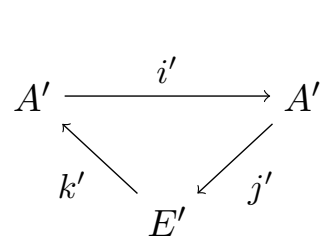

- $A^{\prime}=i(A)$

- $E^{\prime}=H(E)$

- $i^{\prime}(i(a))=i \circ i(a)$

- if $a^{\prime}=i(a)$, then $j^{\prime}(i(a))=[j(a)]_{H(E)}$

- $k^{\prime}[e]=k(e)$

Lemma 1.2.1: $\quad$ The derived couple is itself an exact couple.

Iterating the process of creating derived couples we obtain a sequence of exact couples. Then, calling $E^{r}$ the $E$-term of the $r$-th derived couple, and $d^{r}=j_{r} \circ k_{r}$ the derivative obtained in it, we obtain a spectral sequence $\left\{E^{r}, d^{r}\right\}$.

We will work in this dissertation with very specific types of spectral sequences which were useful tools for calculating homotopy groups with the aid of Eilenberg MacLane spaces. As a starting point, we begin with an intuitive spectral sequence to understand more of how it works and why it can be seen as a tool for approximating homology.

\subsubsection{Spectral sequence of a Filtered Complex}

Our first exploration of spectral sequences will be through the spectral sequence of a filtration. Besides being a good starting point to better understand what is a spectral sequence, it is also an important tool for the construction of the Spectral Sequence of a Fibration, which will be useful for calculating homotopy groups. In this section, we look closely at the spectral sequence for homology with coefficients in an arbitrary group (which will be omitted to facilitate notation), although, with very little adaptations, we can extend the ideas and results for calculating cohomology.

The idea of the spectral sequence of a filtered complex generalises many natural situations in algebraic topology. For instance, if instead of a pair $(X, A)$ we have a sequence $X_{0} \subset X_{1} \subset$ $\cdots \subset X$, then instead of the homology sequence of the pair $(X, A)$, which relates the groups $H_{i}(A), H_{i}(X)$ and $H_{i}(X, A)$, this spectral sequence will give us an algebraic relation between the homology groups of all the $X_{i}$. Analogously, this can be seen as a generalisation of the Meyer-Vietoris sequence applied to a covering by any number of sets.

We start our exploration through the study of a specific problem and a strategy to solve it. Suppose we wish to calculate the homology groups of a given topological space $X$. That is, the homology of the chain complex

$$
\cdots \stackrel{\partial}{\longrightarrow} C_{n+1}(X) \stackrel{\partial}{\longrightarrow} C_{n}(X) \stackrel{\partial}{\longrightarrow} C_{n-1}(X) \stackrel{\partial}{\longrightarrow} \cdots
$$

What we would like to do is to calculate such homology groups "by pieces". For instance, if each $C_{n}$ came with a grading $C_{n}=\bigoplus_{p=1}^{k} C_{n, p}$ with $\partial C_{n, p} \subset C_{n-1, p}$, we could split the chain complex 
above as

$$
\begin{array}{ccc}
\vdots & \vdots \\
\cdots \stackrel{\partial}{\longrightarrow} C_{n+1, p+1}(X) \stackrel{\partial}{\longrightarrow} C_{n, p+1}(X) \stackrel{\partial}{\longrightarrow} C_{n-1, p+1}(X) \stackrel{\partial}{\longrightarrow} \cdots \\
\cdots \stackrel{\partial}{\longrightarrow} C_{n+1, p}(X) \stackrel{\partial}{\longrightarrow} C_{n, p}(X) \stackrel{\partial}{\longrightarrow} C_{n-1, p}(X) \stackrel{\partial}{\longrightarrow} \cdots \\
\cdots \stackrel{\partial}{\longrightarrow} C_{n+1, p-1}(X) \stackrel{\partial}{\longrightarrow} C_{n, p-1}(X) \stackrel{\partial}{\longrightarrow} C_{n-1, p-1}(X) \stackrel{\partial}{\longrightarrow} \cdots
\end{array}
$$

Indeed, the homology groups of the original chain complex would be the direct sums of the homology groups of the new chains above: $H_{d}=\bigoplus_{p=1}^{k} H_{d, p}$.

In this case, one could easily attack the problem of understanding homology by solving the problem in the smaller pieces and putting it together. For instance, if $X$ is a disjoint union of spaces $X_{i}$, we can then see we have one such situation. However, in many natural cases, the structure we want to study in $C_{n}$ is not so well-behaved. More usually, the $C_{n}$ 's are equipped with a filtration:

Definition 1.2.3: A chain complex $\cdots \rightarrow C_{n+1} \rightarrow C_{n} \rightarrow C_{n-1} \rightarrow \ldots$ is endowed with a filtration if, for each $n$, there is a sequence

$$
\begin{gathered}
C_{n,-p}=0 \subset C_{n, 0} \subset C_{n, 1} \subset C_{n, 2} \subset C_{n, 3} \subset \ldots \quad \text { with } \bigcup_{p} C_{n, p}=C_{n} \\
\text { and } \partial C_{n, p} \subset C_{n-1, p}
\end{gathered}
$$

This makes the chain complex above into a filtered complex, and we say it is equipped with the filtration $\left\{C_{n, p}\right\}_{p}$.

Example 1.2.2: $\quad$ A very important example of when a chain complex is endowed with a filtration is obtained when we have a filtration on the space $X$.

$$
0 \subset X_{0} \subset X_{1} \subset X_{2} \subset X_{3} \subset \cdots \subset X \quad \text { with } \bigcup_{p} X_{p}=X
$$

Then setting $C_{n, p}=C_{n}\left(X_{p}\right)$, we obtain a filtration on the chain complex of $X$.

There are many natural filtrations on a space $X$. For instance, if $X$ is a $C W$-complex, we can look at the filtration setting $X_{p}$ equals to the p-skeleton of $X$. However, this is not a very interesting filtration in terms of spectral sequences.

An important example of a filtration, which will be important in the next section, is obtained when we have a fibration $\pi: X \rightarrow B$ over a path connected $C W$-space $B$. Then, if $B^{p}$ is the p-skeleton of $B$, by setting $X_{p}=\pi^{-1}\left(B^{p}\right)$, we obtain a filtration $\left\{X_{p}\right\}$ on $X$, and consequently, also a filtration on the chain complex of $X$. This is a very important example discussed further in the next section.

What we will do is try to adapt the strategy used on the first case above to a filtered complex. 
To do that we will, instead of looking to each $C_{n}$, regard a graded complex which is "similar" to it.

Definition 1.2.4: Given a filtration on a group that is a sequence $0 \subset K_{0} \subset K_{1} \subset \ldots$, with $\bigcup_{p} K_{p}=G$, we define its associated graded group as

$$
G K=\bigoplus_{p} K_{p} / K_{p-1}
$$

The associated graded group approximates the original group using the filtration, by "gluing together" the pieces which are augmented in each step of the filtration. If, for instance, $K$ is a finite dimensional vector space, $G K$ is isomorphic to $K$. However, in the general case, this might not hold. If we consider, for instance, the following filtration of the group $\mathbb{Z}$ :

$$
0 \subset 2 \mathbb{Z} \subset \mathbb{Z}
$$

then the associated graded group is given by

$$
G \mathbb{Z}=\frac{\mathbb{Z}}{2 \mathbb{Z}} \oplus \frac{2 \mathbb{Z}}{0}=\mathbb{Z}_{2} \oplus 2 \mathbb{Z}
$$

which is clearly not isomorphic to $\mathbb{Z}$.

Consequently, we can see that the associated graded group is an approximation which, in the case of our filtered complex, will be well-behaved. Then, our strategy will be to look at the associated graded groups of $C_{n}$ with filtration $\left\{C_{n, p}\right\}_{p}$ :

$$
E_{n}^{0}=\bigoplus_{p} E_{n, p}^{0}, \quad \text { where } E_{n, p}^{0}=\frac{C_{n, p}}{C_{n, p-1}}
$$

Using $\partial$, we can equip $E_{n}^{0}$ with a differential operator $\partial^{0}: E_{n}^{0} \rightarrow E_{n-1}^{0}$, setting

$$
\partial^{0}(\underbrace{c}_{\in C_{n}, p}+C_{n, p-1})=\underbrace{\partial(c)}_{\in C_{n-1, p-1}}+C_{n-1, p-1}
$$

From this we can see that $\partial^{0}\left(E_{n, p}^{0}\right) \subset E_{n-1, p}^{0}$, and we have now a new chain complex which fits in the first case we have studied.

As we have seen, the homology of this associated graded complex can be calculated by pieces, by the direct sum of the homology groups of the complexes 


$$
\begin{aligned}
& \cdots \stackrel{\partial^{0}}{\longrightarrow} E_{n+1, p+1}^{0}(X) \stackrel{\partial^{0}}{\longrightarrow} E_{n, p+1}^{0}(X) \stackrel{\partial^{0}}{\longrightarrow} E_{n-1, p+1}^{0}(X) \stackrel{\partial^{0}}{\longrightarrow} \cdots \\
& \cdots \stackrel{\partial^{0}}{\longrightarrow} E_{n+1, p}^{0}(X) \stackrel{\partial^{0}}{\longrightarrow} E_{n, p}^{0}(X) \stackrel{\partial^{0}}{\longrightarrow} E_{n-1, p}^{0}(X) \stackrel{\partial^{0}}{\longrightarrow} \cdots \\
& \cdots \stackrel{\partial^{0}}{\longrightarrow} E_{n+1, p-1}^{0}(X) \stackrel{\partial^{0}}{\longrightarrow} E_{n, p-1}^{0}(X) \stackrel{\partial^{0}}{\longrightarrow} E_{n-1, p-1}^{0}(X) \stackrel{\partial^{0}}{\longrightarrow} \cdots
\end{aligned}
$$

This can be considered as the page 0 of our spectral sequence, if we think of $E^{0}$ as the direct sum of all $E_{n, p}^{0}$ and $d^{0}: E^{0} \rightarrow E^{0}$ as $\partial^{0}$.

If we set $E_{n, p}^{1}=H_{d}\left(E_{n, p}^{0}\right)=\frac{\operatorname{ker} \partial^{0}: E_{n, p}^{0} \rightarrow E_{n-1, p}^{0}}{\operatorname{Im} \partial^{0}: E_{n+1, p}^{0} \rightarrow E_{n, p}^{0}}$, then we know that $H_{n}\left(E^{0}\right)=\bigoplus_{p} E_{n, p}^{1}$.

We can ask ourselves then if such $H_{d}\left(E^{0}\right)=\bigoplus_{p} E_{n, p}^{1}$ is also the $n$-th homology group of our original complex. But unfortunately, that is not the case. Even if $E_{n}^{0}$ is isomorphic to $C_{n}$, the two are not necessarily isomorphic as chain complexes.

Example 1.2.3: Let us consider the case when each $C_{n}$ is a finite dimensional vector space and is equipped with a filtration $0=C_{n, 0} \subset C_{n, 1} \subset C_{n, 2}=C_{n}$. For notational reasons, we set $C_{n, p}=0$, for $p<0$, and $C_{n, p}=C_{n, 2}$, for $p>2$. So our 0-page has only two non-zero rows and is of the following form:

$$
\begin{aligned}
& \cdots \stackrel{\partial^{0}}{\longrightarrow} E_{n+1,2}^{0}(X) \stackrel{\partial^{0}}{\longrightarrow} E_{n, 2}^{0}(X) \stackrel{\partial^{0}}{\longrightarrow} E_{n-1,2}^{0}(X) \stackrel{\partial^{0}}{\longrightarrow} \cdots \\
& \cdots \stackrel{\partial^{0}}{\longrightarrow} E_{n+1,1}^{0}(X) \stackrel{\partial^{0}}{\longrightarrow} E_{n, 1}^{0}(X) \stackrel{\partial^{0}}{\longrightarrow} E_{n-1,1}^{0}(X) \stackrel{\partial^{0}}{\longrightarrow} \cdots
\end{aligned}
$$

The homology group we want to calculate is $\frac{Z_{n}}{B_{n}}$, where $Z_{n}$ is the set of $\partial$-cycles in $C_{n}$, and $B_{n}$ are the d-boundaries in $C_{n}$.

From the filtration on $C_{n}$ we also obtain the following filtrations

$$
0=Z_{n, 0} \subset Z_{n, 1} \subset Z_{n, 2}=Z_{n} \quad \text { and } \quad 0=B_{n, 0} \subset B_{n, 1} \subset B_{n, 2}=B_{n}
$$

where each $Z_{n, i}=Z_{n} \cap C_{n, i}$ and $B_{n, i}=B_{n} \cap C_{n, i}$.

Thus, we can verify that $\frac{Z_{n}}{B_{n}}=\frac{Z_{n}+C_{n, 1}}{B_{n}+C_{n, 1}} \oplus \frac{Z_{n} \cap C_{n, 1}}{B_{n} \cap C_{n, 1}}=\frac{Z_{n, 2}+C_{n, 1}}{B_{n, 2}+C_{n, 1}} \oplus \frac{Z_{n, 1}}{B_{n, 1}}$.

It would be great if $E_{n, 1}^{1} \cong \frac{Z_{n, 1}}{B_{n, 1}}$ and $E_{n, 2}^{1} \cong \frac{Z_{n, 2}+C_{n, 1}}{B_{n, 2}+C_{n, 1}}$. But that is not the case.

Let us more closely consider those groups: the elements of $E_{n, 1}^{1}$ are of the form $z+I$, where $z$ is a $\partial^{0}$-cycle in $E_{n, 1}^{0}$ (and consequently, in this case, also a $\partial$-cycle in $C_{n, 1}$ ), and $I$ is the image of the map $\partial^{0}: E_{n+1,1}^{0} \rightarrow E_{n, 1}^{0}$. We see that the elements of $E_{n, 1}^{1}$ are precisely $z+I$, where $z \in Z_{n, 1}$. However, $I$ is not equal to $B_{n, 1}$. The problem here is that $B_{n, 1}$ is composed of all the elements of 
$B_{n}$ in $C_{n, 1}$, and this might include the image of elements in $C_{n+1,2}$ which are not being counted in $I$ (since it only considers elements which are images of some element of $C_{n+1,1}$ ). Therefore, $\frac{Z_{n, 1}}{B_{n, 1}}$ is a quotient of $E_{n, 1}^{1}$.

On the other hand, the elements of $E_{n, 2}^{1}$ are of the form $z+\left(B_{n, 2}+C_{n, 1}\right)$, with $z$ on the kernel of $\partial_{0}: E_{n, 2}^{0} \rightarrow E_{n-1,2}^{0}$. This kernel contains not only $\partial$-cycles, but also elements which are taken by $\partial$ to $C_{n-1,1}$. Hence, $\frac{Z_{n, 2}+C_{n, 1}}{B_{n, 2}+C_{n, 1}}$ is a subspace of $E_{n, 2}^{1}$.

Therefore, $E^{1}$ is bigger than the homology of our initial complex. We will need a better refinement.

Intuitively, the problem with our approximation of the homology group is that the associated graded complex only detects activities which occur on the same "horizontal level". However, on our original complex, $\partial$ could carry elements from $C_{n, p}$ to $C_{n-1, q}, q<p$, which are not considered here.

We consider those elements which are being moved to another level on the next pages of our spectral sequence. To construct page $E^{1}$, we consider the elements which are carried down precisely one level.

Notice that $\partial$ induces a natural map $\partial^{1}: E_{n, p}^{1} \rightarrow E_{n-1, p-1}^{1}$, since, for every element $[e] \in E_{n, p}^{1}$, the boundary $\partial(e)$ is a cycle in $C_{n-1, p-1}$, and therefore, defines an element in $E_{n-1, p-1}^{1}$. Thus, our 1-page looks like

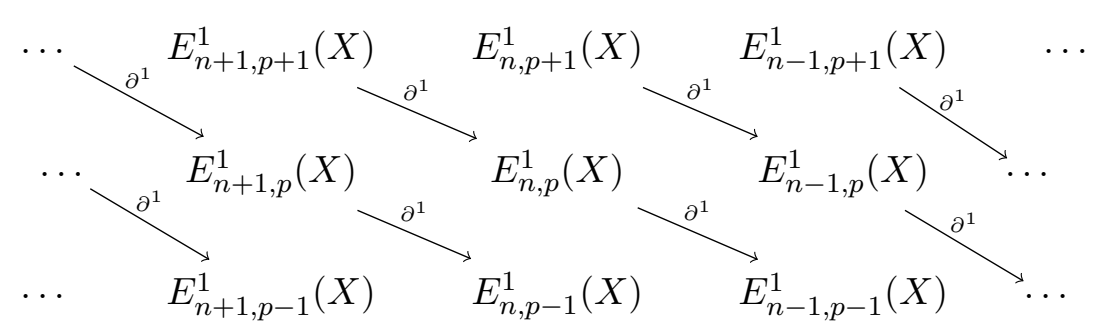

We then define $E_{n, p}^{2}=H_{n}\left(E_{n, p}^{1}\right)=\frac{\operatorname{ker} \partial^{1}: E_{n, p}^{1} \rightarrow E_{n-1, p-1}^{1}}{\operatorname{Im} \partial^{1}: E_{n+1, p+1}^{1} \rightarrow E_{n, p}^{1}}$.

From the discussion above, we see that $E^{2}$ is a better approximation of the homology group of the original chain complex.

We return to the example to see what happens in the second page of our spectral sequence.

Example 1.2.4: The page 1 of the example 1.2.3, is of the form 


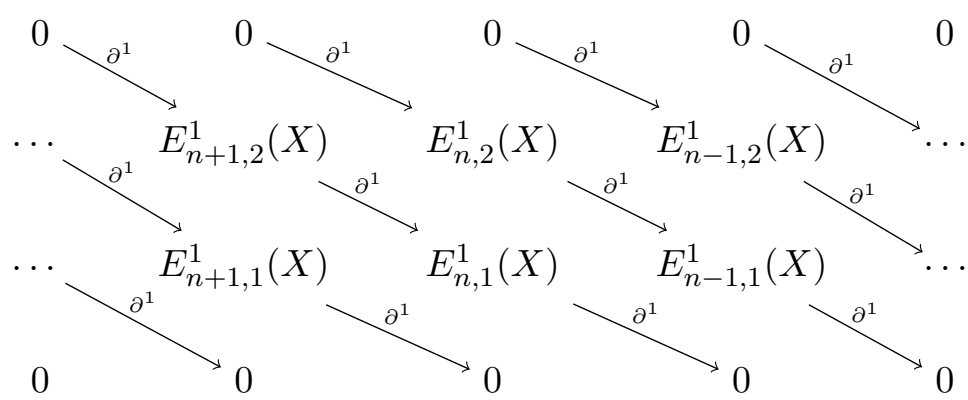

$E_{n, 1}^{2}$ is precisely the quotient of $E_{n, 1}^{1}$ by the image, $I$, of $\partial^{1}: E_{n+1,2}^{1} \rightarrow E_{n, 1}^{1}$. Since I gives all the boundaries that lie in $C_{n, 1}$ we take off exactly the spare elements in $E_{n, 1}^{1}$. Therefore, $E_{n, 1}^{2}$ is precisely $\frac{Z_{n, 1}}{B_{n, 1}}$.

On the other hand, $E_{n, 2}^{2}$ is the kernel of the map $\partial^{1}: E_{n, 2}^{1} \rightarrow E_{n-1,1}^{1}$, which consists of all the elements whose boundary equals a boundary of some element of $C_{n, 1}$. Then $E_{n, 2}^{2}$ is isomorphic to $\frac{Z_{n, 2}+C_{n, 1}}{B_{n, 2}+C_{n, 1}}$.

Therefore, $E_{n, 1}^{2} \oplus E_{n, 2}^{2}$ is precisely the $n$-th homology group of our original complex.

In the general case, $E^{2}$ will still be an approximation of the desired homology group, because we have not yet considered elements which were carried more than one level down. We refine such approximation on the next pages of our spectral sequence: on page $E^{r}$ we use $\partial$ to define a differential map $\partial^{r}: E_{n, p}^{r} \rightarrow E_{n-1, p-r}^{r}$ which will look at the elements carried out $r$ rows. Page 2 of a spectral sequence is then

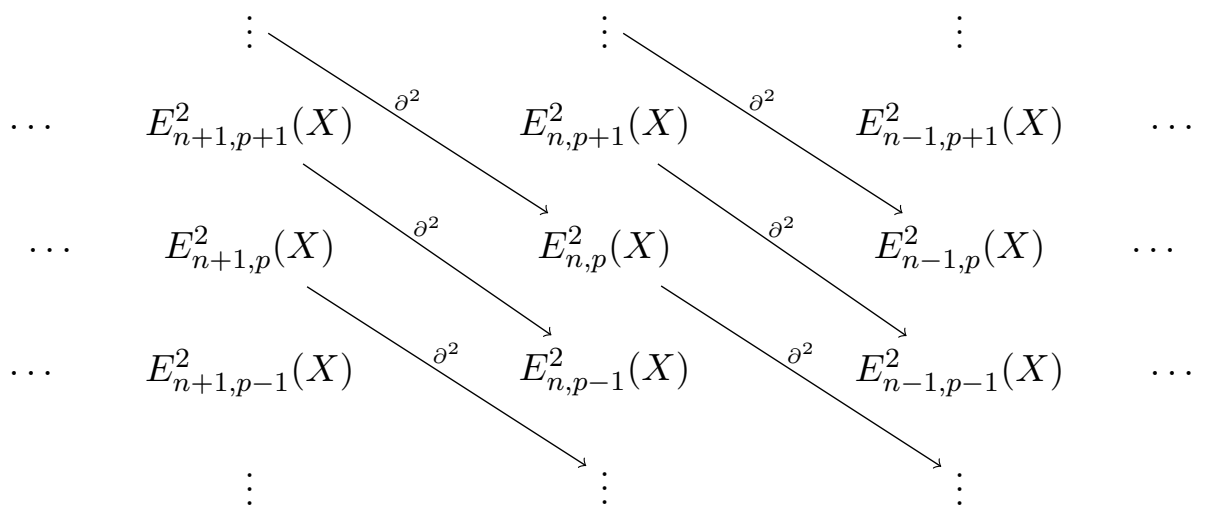

Example 1.2.5: Let us take a final look at example 1.2.3 to see how the spectral sequence evolves. From the discussion above, we know that page 2 will be of the form 


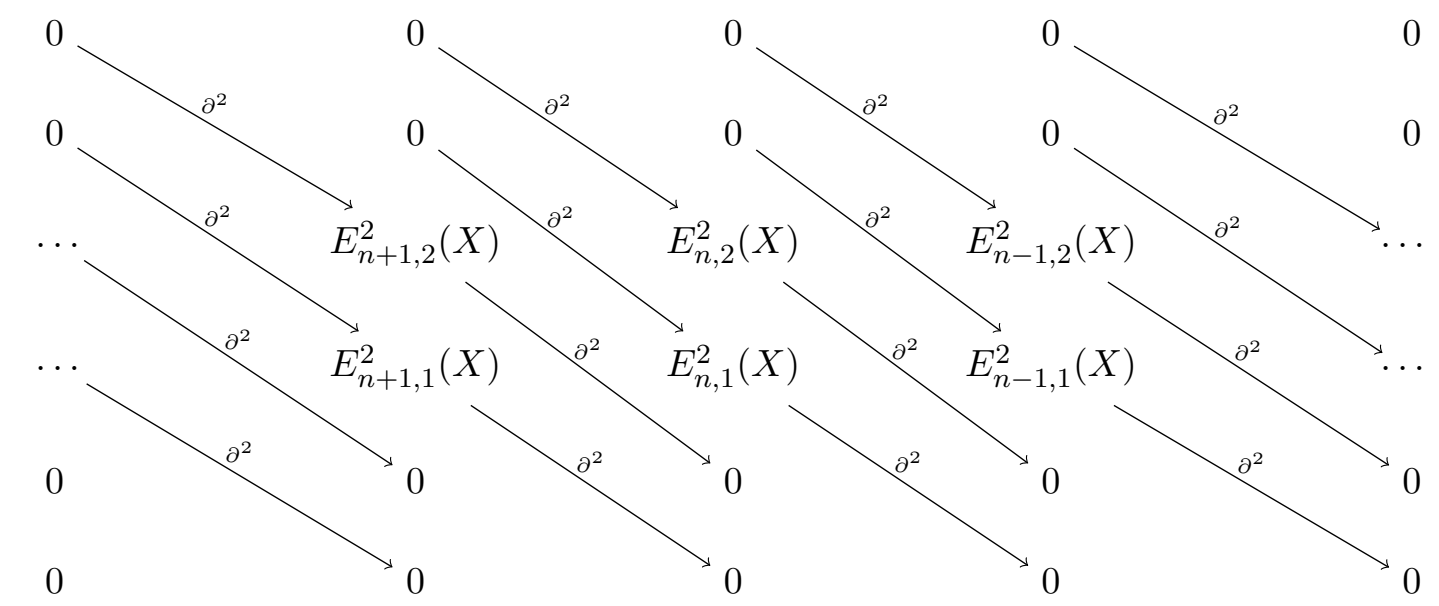

Therefore the terms $E_{n, p}^{3}$ will be precisely $E_{n, p}^{2}$. The differential $\partial^{3}$ will go down 3 rows and all arrows will again either arrive or go to zeroes, which implies that the $E^{4}$ terms are the same ones of $E^{2}$. This episode is repeated in all the other pages and therefore, the sequence stabilises after the second page.

Now that we have exposed the idea behind the spectral sequence of a filtered complex, we will move to the formal definitions making use of exact couples. We will then prove that, in good cases like the one in the example above, the spectral sequence will indeed recover the homology of our original complex.

From a chain complex equipped with a filtration $\left\{C_{n, p}\right\}$, we get, for each $p$, an exact sequence of chain complexes:

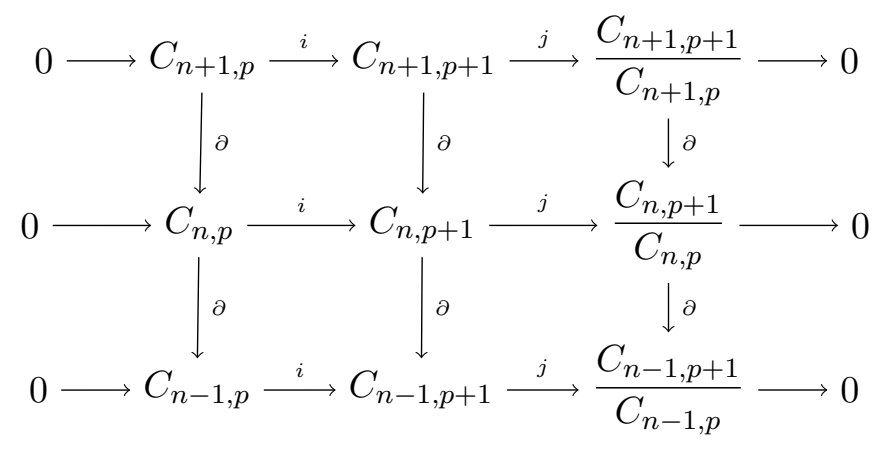

and then, for each $p$, a long exact sequence in homology

$$
\cdots \rightarrow H\left(\frac{C_{n+1, p+1}}{C_{n+1, p}}\right) \stackrel{k_{1}}{\rightarrow} H\left(C_{n, p}\right) \stackrel{i_{1}}{\rightarrow} H\left(C_{n, p+1}\right) \stackrel{j_{1}}{\rightarrow} H\left(\frac{C_{n, p+1}}{C_{n, p}}\right) \stackrel{k_{1}}{\rightarrow} H\left(C_{n-1, p}\right) \rightarrow \ldots
$$

Because of the discussion above, we want the term $E$ of our exact couple to be related to the successive quotients of our filtration. It is natural, then, to use the following notations 
$A_{n, p}^{1} \doteq H\left(C_{n, p}\right), E_{n, p}^{1} \doteq H\left(\frac{C_{n+1, p+1}}{C_{n+1, p}}\right)$ and define

$$
A^{1}=\bigoplus_{n, p} A_{n, p}^{1} \quad E^{1}=\bigoplus_{n, p} E_{n, p}^{1}
$$

What we have is an exact couple depicted on the right. It might be difficult to visualise such an exact couple because the arrows are not all defined in only one long exact sequence, as in the first example (Example 1.2 .1 . For instance, in sequence 1.2 , we have information of $i_{1}$ on $A_{n, p}^{1}$

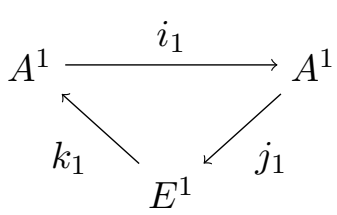
but we cannot know how $j_{1}$ is defined on $A_{n, p}^{1}$. This information will be obtained on the long exact sequence related to $p-1$. To see how they interact, we arrange all exact homology sequences the following way, known as "staircase diagram":

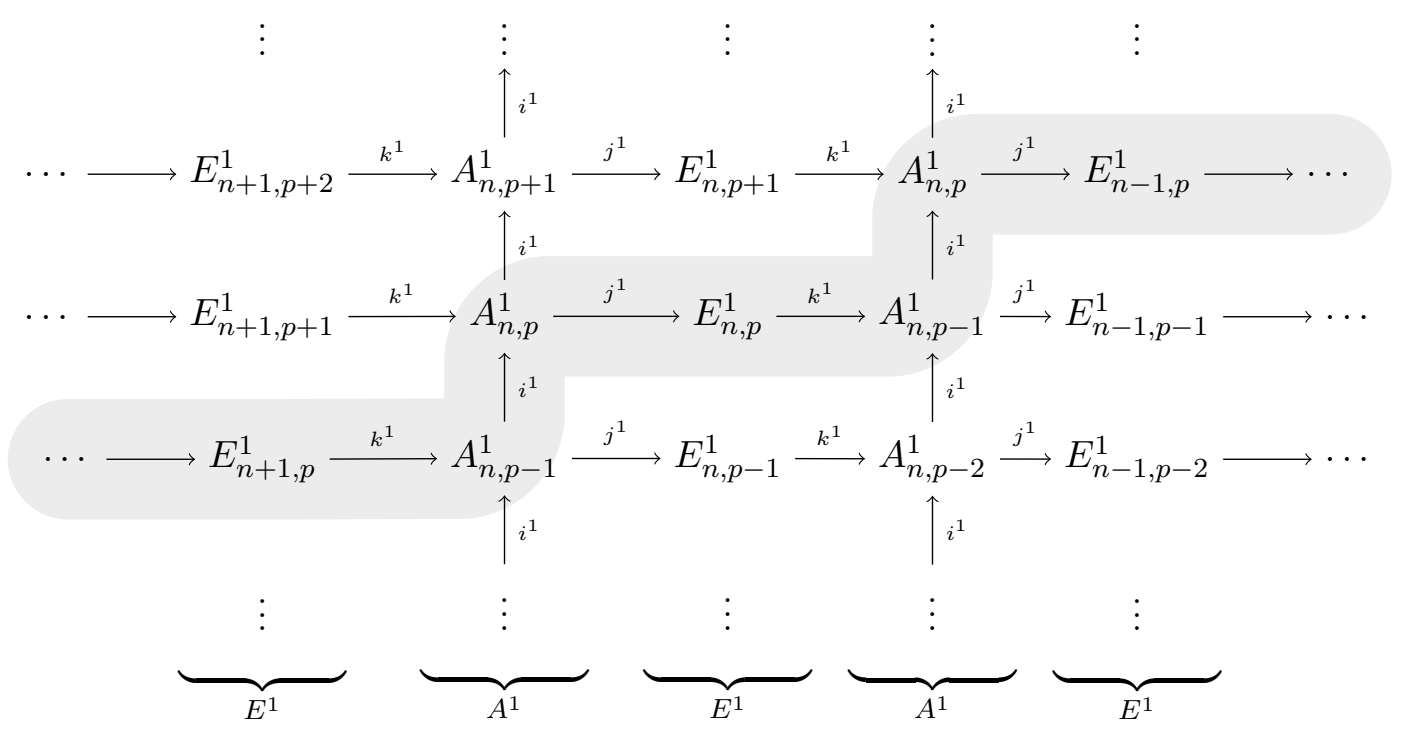

The dashed "staircase" clarifies how each long exact sequence is arranged in such a diagram. This is our first exact couple and, equipped with the differential $d^{1}=j_{1} \circ k_{1}$, this defines the first page of our spectral sequence. Notice that, from the definition of the boundary operator (which is denoted $k_{1}$ ) in the long exact sequence of homology, the differential $d^{1}$ is precisely the one we defined in the discussion above. Notice also that, in the staircase diagram, the arrows $d^{1}$ are horizontal because of the way we organised the terms in the diagram, which is canonical in the literature. However, we see from the definition that $d^{1}$ is precisely the map we defined previously.

When we pass to the derived couple, we obtain exactly the relation we desired before: each group $A_{n, p}^{1}$ is replaced by $A_{n, p}^{2}$, which is the image of $i_{1}: A_{n, p-1}^{1} \rightarrow A_{n, p}^{1}$ (the image of the term directly below $A_{n, p}^{1}$ under the vertical map $i_{1}$ ). Then, setting $d^{1}=j_{1} \circ k_{1}$, we have $E_{n, p}^{2}=\frac{\operatorname{ker} d^{1}: E_{n, p}^{1} \rightarrow E_{n-1, p}^{1}}{\operatorname{Im} d^{1}: E_{n+1, p}^{1} \rightarrow E_{n, p}^{1}}$. Considering the relation $j_{2}\left(i_{1}(a)\right)=\left[j_{1}(a)\right]$, from the definition of the derived couple, we see that page 2 of our spectral sequence is of the following form: 


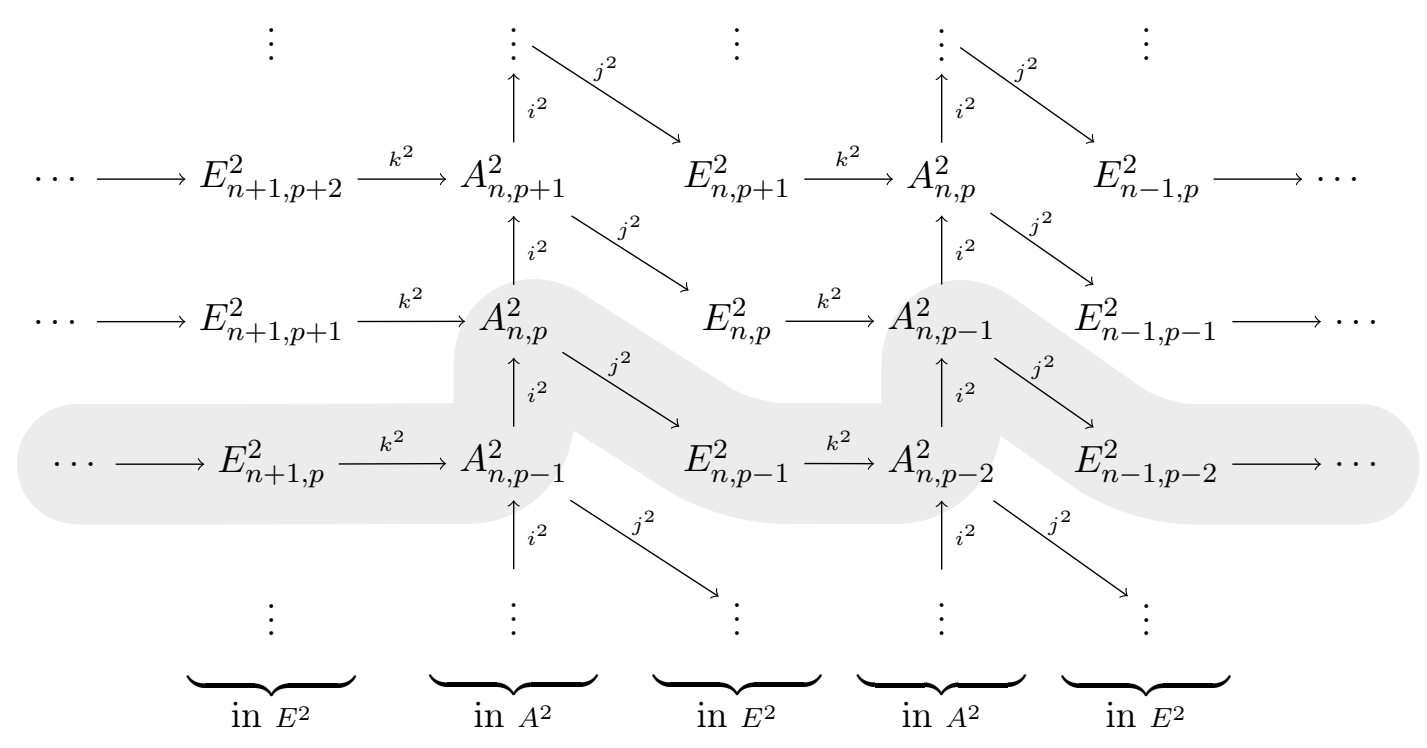

Again, the dashed part indicates how the exact couple is organised in such a diagram. We see that, as expected from the previous discussion, the differentials $d^{2}=j_{2} \circ k_{2}$ go one row down. We also see that, by repeating the process of taking the derived couple, the arrows $j_{n}$ will go $n-1$ rows down, and therefore, so will $d^{n}$.

Example 1.2.6: It is important for us to consider the case when the filtration on the chain complex comes from a filtration $\emptyset \subset X_{0} \subset X_{1} \subset \ldots$ on the space $X$, as described in Example 1.2.2. Then each $C_{n, p}$ is actually $C_{n}\left(X_{p}\right)$, and therefore

$$
A_{n, p}^{1}=H_{n}\left(X_{p}\right) \quad E_{n, p}^{1}=H_{n}\left(X_{p}, X_{p-1}\right)
$$

We also see that the staircase diagram is composed of all exact sequences of the pairs $\left(X_{p}, X_{p-1}\right)$.

Having formalised the definition of spectral sequence of a filtration, we now seek to understand precisely how and under what circumstances the original homology is recovered. We observed in Example 1.2.4 that there are some cases in which the pages stabilise and the homology of our original complex $H_{n}$ can be somehow recovered from such pages. In such example, we had a very "short" filtration with $C_{n, 2}=C_{n}$ for all $n$. This condition, that the filtration terminates in a finite number of steps, is what helps us "recover" the original homology, although we will need less than what was asked for in Example 1.2.4. What we ask is that: for all $n$, there exists $l>0$ such that $C_{n, p}=C_{n}$, for all $p \geq l$.

What the condition above means is that all but finitely many of the maps in the A-column are isomorphisms. At the "top" of each such columns there is a stable value, which we denote as $A_{n, \infty}^{1}$, and is precisely the $n$-th homology group of our original complex. By exactness of the exact couple, we see that this is equivalent to saying that only finitely many terms in the E-column are non-zero.

Example 1.2.7: If we consider when the filtration on the chain complex is induced by a filtration on the space $X$, the condition above is asking that, for all $n$, the inclusion $X_{p} \hookrightarrow X$ induces an isomorphism on $H_{n}$, for a sufficiently large $p$. In this case, we see that $A_{n, \infty}^{1}=H_{n}(X)$. 
Since the differentials $d^{r}: E_{n, p}^{r} \rightarrow E_{n-1, p-r}^{r}$ go $r-1$ rows down on the diagram, under the condition above, we see that all the differentials into and out of a given E-column must be zero for a sufficiently large $r$. This means that, for all $n$ and $p$, the terms $E_{n, p}^{r}$ become stationary for sufficiently large $r$.

Definition 1.2.5: If the terms $E_{n, p}^{r}$ eventually became stationary, we denote its value by $E_{n, p}^{\infty}$. If each $E_{n}^{\infty}$ is equal to the associated graded group of some filtered group $H$, then the spectral sequence converges to $H$.

Finally, we establish a result on the convergence of our spectral sequence:

Theorem 1.2.2: If all but finitely of the maps in the A-column are isomorphisms, the stable group $E_{n, p}^{\infty}$ is isomorphic to the quotient $F_{n}^{p} / F_{n}^{p-1}$ for the filtration $\cdots \subset F_{n}^{p-1} \subset F_{n}^{p} \subset \ldots$ of $A_{n, \infty}^{1}$ by the subgroups $F_{n}^{p}=\operatorname{Im}\left(A_{n, p}^{1} \rightarrow A_{n, \infty}^{1}\right)$. In other words, the spectral sequence converges to $A^{\infty}$, the homology of our original complex.

Proof: If we look at the $r$-th page of our spectral sequence, we see the term $E_{n, p}^{r}$ embedded in an exact sequence:

$$
E_{n+1, p+r-1}^{r} \stackrel{k_{r}}{\longrightarrow} A_{n, p+r-2}^{r} \stackrel{i_{r}}{\longrightarrow} A_{n, p+r-1}^{r} \stackrel{j_{r}}{\longrightarrow} E_{n, p}^{r} \stackrel{k_{r}}{\longrightarrow} A_{n-1, p-1}^{r} \stackrel{i_{r}}{\longrightarrow} A_{n-1, p}^{r} \stackrel{j_{r}}{\longrightarrow} E_{n-1, p-r+1}^{r}
$$

Since all but finitely many terms in the E-column are non-zero, as $r$ gets bigger, eventually the first and last terms in the sequence above will be zero.

Alternatively, since $C_{n, p}=0$ for all negative integers $p$, we know that $A_{n, p}^{1}=0$ for all $p<0$. By definition, $A_{n, p}^{r}$ is seen as the image of $A_{n, p-r}^{1}$ under the inclusion into $A_{n, p}^{1}$ and therefore, for a sufficiently large $r$, it will also be trivial. This implies that the last two $A$ terms in the sequence above will also become zero for a sufficiently large $r$.

We see, from the sequence above, that $E_{n, p}^{r}$ is isomorphic to the quotient $A_{n, p+r-1}^{r} / i\left(A_{n, p+r-2}^{r}\right)$, or, in other words, $i^{r-1}\left(A_{n, p}^{1}\right) / i^{r}\left(A_{n, p-1}^{1}\right)$, a quotient of subgroups of $A_{n, p+r-1}^{1}=A_{n, \infty}^{1}$.

If $F_{n}^{p}$ is the image of the map $A_{n, p}^{1} \rightarrow A_{n, \infty}^{1}$, we have shown that $E_{n, p}^{\infty}$ is isomorphic to $F_{n}^{p} / F_{n}^{p-1}$.

The result above gives us a condition in which our spectral sequence converges to the homology of our original complex. However, what we obtain are graded groups $G H_{n}$ associated to each homology group $H_{n}$. We know that $G H_{n}$ is not necessarily isomorphic to $H_{n}$, but one could hope that it would be possible to recover the original group from its associated graded group. However, it is not the case. For example, the two groups $\mathbb{Z}_{2} \oplus \mathbb{Z}_{2}$ and $\mathbb{Z}_{4}$ filtered by

$$
\mathbb{Z}_{2} \subset \mathbb{Z}_{2} \oplus \mathbb{Z}_{2} \quad \text { and } \quad \mathbb{Z}_{2} \subset \mathbb{Z}_{4}
$$

have isomorphic associated graded groups, though they are not themselves isomorphic. This shows that, even with such equipment, in some cases, it is still not sufficient to give a precise account of the homology of our original complex. 
This intricate tool, however confusing, is very important for the construction of the Spectral Sequence of a Fibration, which yields many important results and which is covered in next section.

\subsubsection{Spectral Sequence of a Fibration}

The spectral sequence addressed in this section is one of the most important tools in the computation of homotopy groups. We can trace the motivation for this construction to Eilenberg's article On The Problems of Topology [Eil49], which provided a list of questions, including:

Problem 17. What are the relations connecting the homology structure of the bundle, base space, fiber and group?

It was Serre, in Singular Homology of Fiber Spaces [Ser12], who provided the first answer to this question, by constructing what became known as Serre's Spectral Sequence, and which is the topic of this section.

We proceed with this construction by looking at the spectral sequence of a filtered complex, obtained when we have a well-behaved fibration and when we endow the total space with a filtration which is nicely inherited from the base space. This filtration, which we have already defined in Example 1.2.2, is very useful because its second page is of a very specific form which depends only on the homology groups of the fiber and the base space. This makes it much more computable and a very useful tool.

Let us recall the filtration we use. Let $\pi: X \rightarrow B$ be a fibration with $B$ a path-connected CW-complex. We define a filtration on $X$, from the filtration of $B$ given by its skeletons, setting $X_{p}=\pi^{-1}\left(B^{p}\right)$. Since $\left(B, B^{p}\right)$ is $p$-connected, the homotopy lifting property implies that $\left(X, X_{p}\right)$ is also $p$-connected. Then, from the long exact sequence of the pair

$$
\cdots \rightarrow H_{n+1}\left(X, X_{p} ; G\right) \rightarrow H_{n}\left(X_{p} ; G\right) \rightarrow H_{n}(X ; G) \rightarrow H_{n}\left(X, X_{p} ; G\right) \rightarrow \ldots
$$

the inclusion $X_{p} \hookrightarrow X$ induces isomorphism on $H_{n}(\cdot ; G)$, if $n<p$, for any group $G$. So, together with the fact that $X_{p}=\emptyset$ for $p<0$ it is enough by Theorem 1.2 .2 , to guarantee that the spectral sequence with coefficients in $G$ associated with this filtration converges to $H_{*}(X ; G)$.

What we now do is a change in notation which simplifies this special type of spectral sequence. We know that the terms of the first page are of the form $E_{n, p}^{1}=H_{n}\left(X_{p}, X_{p-1} ; G\right)$ and that these are non-zero only if $n \geq p$, since $\left(X_{p}, X_{p-1}\right)$ inherits $(p-1)$-connectedness from $\left(B^{p}, B^{p-1}\right)$. In this case it is useful to change notation setting $n=p+q$ and use the parameters $p$ and $q$. Now our first page is composed of terms $E_{p, q}^{1}=H_{p+q}\left(X_{p}, X_{p-1} ; G\right)$, which are non-zero only if $p, q \geq 0$, and the terms corresponding to $E_{n}^{r}$ are the direct sum of the elements $E_{p, q}^{r}$ with $p+q=n$. In the old notation, if the differentials were of the form $d^{r}: E_{n, p}^{r} \rightarrow E_{n-1, p-r}^{r}$; in the changed notation, we get $d^{r}: E_{p, q}^{r} \rightarrow E_{p-r, q+r-1}^{r}$.

Now we can think of the pages organised as follows: 

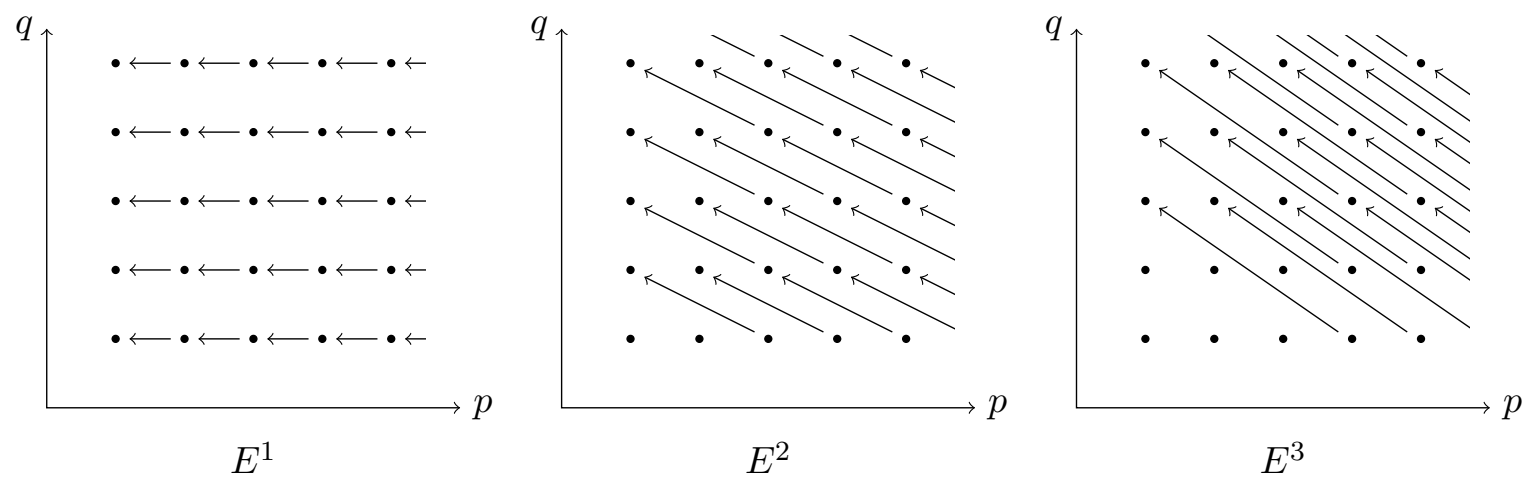

The special fact about this spectral sequence is that, under a good condition on the fibration, the terms of the second page have an explicit formulation in terms of the homology groups of the base space and the fiber. This good condition of the fibration is that $\pi_{1}(B)$ acts trivially on $H_{*}(F ; G)$. We now explain this action in detail.

Recall that, under the hypothesis that $B$ is path-connected, we know that all fibers $F_{b}=\pi^{-1}(b)$ are homotopy equivalent to a fixed fiber $F$, since each path $\gamma: I \rightarrow B$ lifts to a homotopy equivalence $L_{\gamma}: F_{\gamma(0)} \rightarrow F_{\gamma(1)}$ between the fibers over the endpoints of $\gamma$. If we restrict $\gamma$ to the loops at the basepoint of $B$, then we obtain homotopy equivalences $L_{\gamma}: F \rightarrow F$ for the fiber over the basepoint. This association $\gamma \rightarrow L_{\gamma}$ actually respect the equivalences and operations of $\pi_{1}(B)$, and therefore when we take the induced homomorphism on the homology group of $F$, the association $\gamma \rightarrow L_{\gamma *}$ defines an action of $\pi_{1}(B)$ on $H_{*}(F ; G)$. The condition we impose on the fibration is that this action is trivial, that is, $L_{\gamma^{*}}$ is the identity in $H_{*}(F ; G)$, for all loops $\gamma$.

Theorem 1.2.3: $\quad$ Let $F \rightarrow X \rightarrow B$ be a fibration with $B$ path-connected. If $\pi_{1}(B)$ acts trivially on $H_{*}(F ; G)$, then there is a spectral sequence $\left\{E_{p, q}^{r}, d^{r}\right\}$ with

(a) $d^{r}: E_{p, q}^{r} \rightarrow E_{p-r, q+r-1}^{r}$ and $E_{p, q}^{r+1}=\frac{\operatorname{ker} d^{r}}{\operatorname{Im} d^{r}}$ at $E_{p, q}^{r}$.

(b) stable terms $E_{p, n-p}^{\infty}$ isomorphic to the successive quotients $\frac{F_{n}^{p}}{F_{n}^{p-1}}$ in a filtration

$$
0 \subset F_{n}^{0} \subset \cdots \subset F_{n}^{n}=H_{n}(X ; G) \quad \text { of } H_{n}(X ; G) .
$$

(c) $E_{p, q}^{2} \approx H_{p}\left(B ; H_{q}(F: G)\right)$.

If the hypothesis about the triviality of the action of $\pi_{1}(B)$ on $H_{*}(F ; G)$ fails, we still have an analogous and useful result. The only difference is that now $E_{p, q}^{2}$ is isomorphic to the homology group $H_{p}\left(B ; H_{q}(F ; G)\right)$ with twisted coefficients, which are not discussed in this project.

An extreme case is seen when $X$ is contractible, for instance if we have the path space fibration $\Omega X \rightarrow P X \rightarrow X$. We discuss one such example and see how this spectral sequence gives us information.

Example 1.2.8: Using the spectral sequence of the path fibration over spheres $S^{n}$, we can compute the homology groups of the loopspaces $\Omega S^{n}$.

The case $n=1$ is the only one which is not clearly in the hypothesis of Theorem 1.2.3, since $S^{1}$ has a non-trivial fundamental group. However, this case is fairly simple, since $\Omega S^{1}$ has 
contractible components, as one can see by lifting loops to the universal cover of $S^{1}$.

For $n \geq 2$, the pathspace fibration $\Omega S^{n} \rightarrow$ $P\left(S^{n}\right) \rightarrow S^{n}$ satisfies the hypothesis of the theorem above, thus we have a Serre spectral sequence for homology. The terms on its second page are of the form $E_{p, q}^{2}=H_{p}\left(S^{n} ; H_{q}\left(\Omega S^{n}\right)\right)$, therefore, we have only two non-trivial columns, $p=0$ and $p=n$, and the elements of such columns are precisely the homology groups of $\Omega S^{n}$.

Since the pathspace is contractible, we know that the $E^{\infty}$ must be trivial, with just $\mathbb{Z}$ in the $(0,0)$

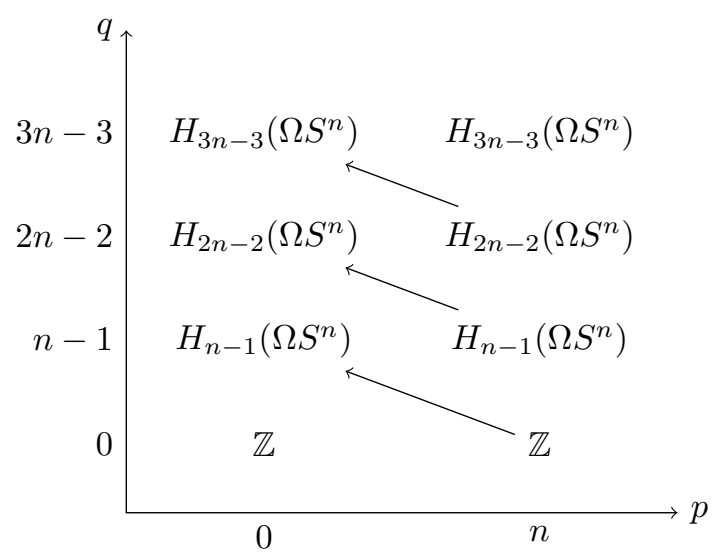
position. The only differential which can be nonzero is $d^{n}$, which relates the elements in columns 0 and $n$. Since all these groups must vanish on $E^{\infty}$, the differentials $d^{n}$ must be isomorphisms (except the ones related to the $(0,0)$ position), and therefore, proceeding by induction, we see that $H_{i}(\Omega ; \mathbb{Z})$ is $\mathbb{Z}$ if $(n-1) \mid i$ and zero otherwise.

As another application of the spectral sequence of a path-fibration, we deduce one of the great results connecting homotopy and homology groups of spaces:

Example 1.2.9: $\quad$ The Hurewicz Isomorphism Theorem is a very important result in Algebraic Topology. In dimension 1 it states that, if $X$ is a path-connected space, then $H_{1}(X)$ is the abelianization of $\pi_{1}(X)$. We use the spectral sequence of the path space fibration to prove Hurewicz Isomorphism Theorem for dimensions greater than 1 :

Theorem 1.2.4: $\quad$ Let $X$ be a simply connected path-connected $C W$-complex. Then the first nontrivial homotopy and homology groups occur in the same dimension and are equal, i.e., given a positive integer $n \geq 2$, if $\pi_{q}(X)=0$ for $1 \leq q<n$, then $H_{q}(X)=0$ for $1 \leq q<n$ and $H_{n}(X)=\pi_{n}(X)$.

We prove this result by induction using the path space fibration. For the case $n=2$, the second page of this spectral sequence is

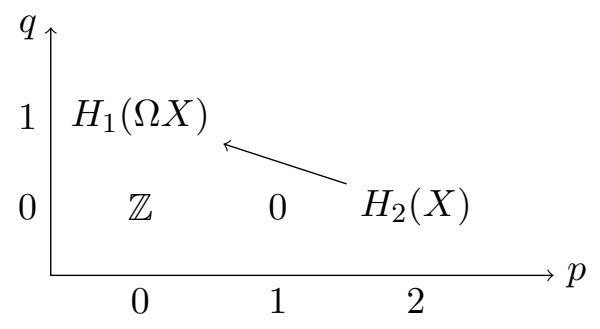

Since $P X$ is contractible, we know that

$$
0=G H_{1}(P X)=E_{1,0}^{\infty} \oplus E_{0,1}^{\infty} \text { and } 0=G H_{2}(P X)=E_{2,0}^{\infty} \oplus E_{1,1}^{\infty} \oplus E_{0,2}^{\infty}
$$

In order for that to happen, the terms $E_{0,1}^{2}$ and $E_{2,0}^{2}$ must vanish at some point of this spectral sequence and thus the correspondent terms on page $E^{3}$ must already be zero because all the $d^{r}$ entering and leaving such terms will be trivial after the second page. In order for that to occur, 
we need the arrow in the diagram above to be an isomorphism. Therefore

$$
H_{2}(X)=H_{1}(\Omega X)=\pi_{1}(\Omega X)=\pi_{2}(X)
$$

If $n$ is any positive integer greater than 2 , we can apply the induction hypothesis to the space $\Omega X$ and we see that

$$
H_{q}(\Omega X)=0, \quad \text { for } q<n-1
$$

and

$$
H_{n-1}(\Omega X)=\pi_{n-1}(\Omega X)=\pi_{n}(X)
$$

Then, the $E^{2}$ page of the spectral sequence of the path fibration is of the form:

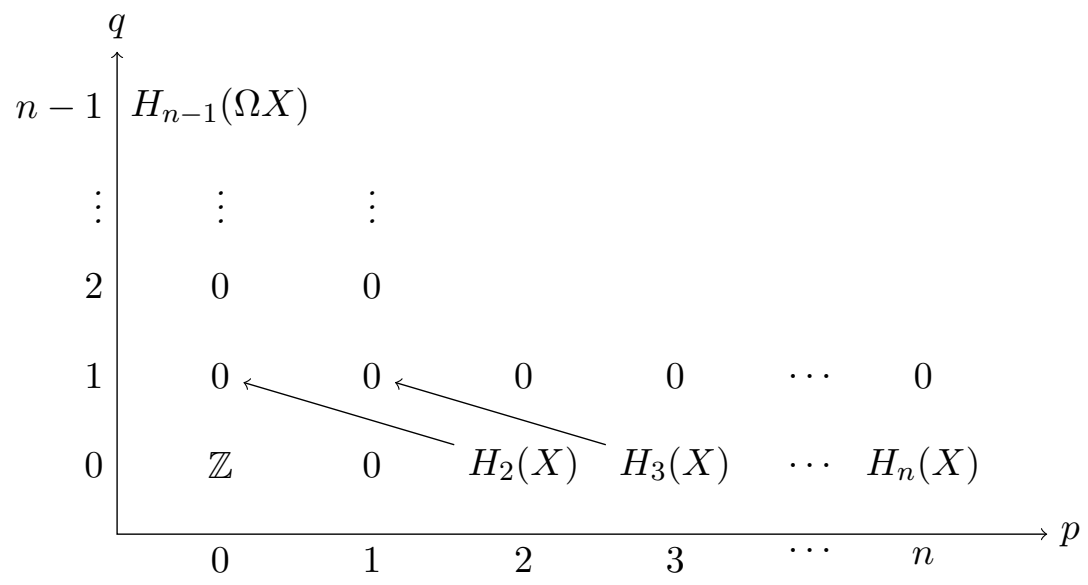

Again, since PX has trivial homology, we conclude that the only group which will not vanish in this spectral sequence is $\mathbb{Z}$ in position $(0,0)$. We can see that the term $E_{2,0}^{2}=H_{2}(X)$ must vanish after the second page, and therefore, the diagram above shows us it must be the trivial group. Analogously, we see that the term $E_{3,0}^{2}=H_{3}(X)$ must vanish after the third page and therefore, since both $d^{2}$ and $d^{3}$ to and from this term are the zero map, we conclude that $H_{3}(X)$ must already be 0. By this same argument, we get

$$
H_{q}(X)=0, \quad \text { for } 1 \leq q<n
$$

Both the terms $E_{0, n-1}^{2}=H_{n-1}(\Omega X)$ and $E_{n, 0}^{2}=H_{n}(X)$ will also vanish, and this only happens through the differential $d^{n}$ which relates to both of them

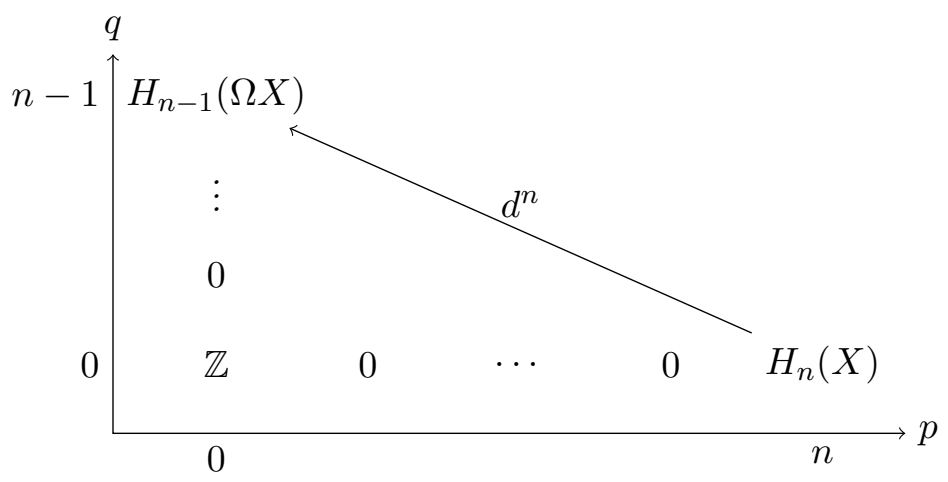

Then such arrow must be an isomorphism, and therefore,

$$
H_{n}(X)=H_{n-1}(\Omega X)=\pi_{n}(X)
$$


As an immediate consequence of the theorem above, we have a proof that the first $n$ homotopy groups of the sphere $S^{n}$ are trivial and $\pi_{n}\left(S^{n}\right)=\mathbb{Z}$.

Example 1.2.10: Let $k$ be a field and $F \rightarrow X \rightarrow B$ a fibration satisfying the hypothesis of Theorem 1.2.3. We see that if both $H_{*}(B ; k)$ and $H_{*}(F ; k)$ are finite dimensional vector spaces, then $H_{*}(X ; k)$ is also finite dimensional and the Euler characteristics of such spaces over $k$ are related by $\chi(X)=\chi(B) \cdot \chi(F)$. We construct the result using the partial sums

$$
\chi_{l}\left(E_{*, *}^{i}\right)=\sum_{n=0}^{l}(-1)^{n} \operatorname{dim}\left(\bigoplus_{p+q=n} E_{p, q}^{i}\right)
$$

Notice that $\lim _{l \rightarrow \infty} \chi_{l}\left(E_{*, *}^{i}\right)=\chi\left(E_{*, *}^{i}\right)$ and $\chi\left(E_{*, *}^{\infty}\right)=\chi(X)$.

Since we are considering coefficients in a field, the Kunneth formula gives us that $H_{p}(B ; k) \otimes$ $H_{q}(F ; k) \approx H_{p}\left(B ; H_{q}(F ; k)\right) \approx E_{p, q}^{2}$ and therefore,

$$
\begin{aligned}
\chi\left(E_{p, q}^{2}\right) & =\lim _{l \rightarrow \infty} \sum_{n=0}^{l}(-1)^{n} \operatorname{dim}\left(\bigoplus_{p+q=n} H_{p}(B ; k) \otimes H_{q}(F ; k)\right) \\
& =\lim _{l \rightarrow \infty} \sum_{n=0}^{l}\left(\sum_{p+q=n}(-1)^{p+q} \operatorname{dim}\left(H_{p}(B ; k)\right) \cdot \operatorname{dim}\left(H_{q}(F ; k)\right)\right) \\
& =\chi(B) \cdot \chi(F)
\end{aligned}
$$

What we do now is show that $\chi\left(E_{*, *}^{i}\right)=\chi\left(E_{*, *}^{i+1}\right)$ for an arbitrary $i$, which will give us the result. Recall from linear algebra that:

$$
\begin{aligned}
\operatorname{dim}\left(E_{p, q}^{i}\right) & =\operatorname{dim}\left(\operatorname{ker} d_{p, q}^{i}\right)+\operatorname{dim}\left(\operatorname{Im} d_{p, q}^{i}\right) \\
\operatorname{dim}\left(E_{p, q}^{i+1}\right) & =\operatorname{dim}\left(\operatorname{ker} d_{p, q}^{i}\right)-\operatorname{dim}\left(\operatorname{Im} d_{p+i, q-i+1}^{i}\right)
\end{aligned}
$$

Thus, we can see that

$$
\begin{aligned}
\chi_{l}\left(E_{*, *}^{i}\right)= & \sum_{n=0}^{l} \sum_{p+q=n}\left[(-1)^{n} \operatorname{dim}\left(\operatorname{ker} d_{p, q}^{i}\right)+(-1)^{n} \operatorname{dim}\left(\operatorname{Im} d_{p, q}^{i}\right)\right] \\
= & \sum_{n=0}^{l}\left[\sum_{p+q=n}(-1)^{n} \operatorname{dim}\left(\operatorname{ker} d_{p, q}^{i}\right)+\sum_{r+s=n}(-1)^{n} \operatorname{dim}\left(\operatorname{Im} d_{r, s}^{i}\right)\right] \\
= & \operatorname{dim}\left(\operatorname{ker} d_{0,0}^{i}\right)+\sum_{n=1}^{l}\left[\sum_{p+q=n}(-1)^{n} \operatorname{dim}\left(\operatorname{ker} d_{p, q}^{i}\right)+\sum_{r+s=n-1}(-1)^{n} \operatorname{dim}\left(\operatorname{Im} d_{r, s}^{i}\right)\right]+ \\
& \quad+\sum_{r+s=n}(-1)^{l} \operatorname{dim}\left(\operatorname{Im} d_{r, s}^{i}\right) \\
= & \sum_{n=0}^{l}(-1)^{n} \operatorname{dim}\left(\bigoplus_{p+q=n} E_{p, q}^{i+1}\right)+\sum_{r+s=n}(-1)^{l} \operatorname{dim}\left(\operatorname{Im} d_{r, s}^{i}\right) \\
= & \chi_{l}\left(E_{*, *}^{i+1}\right)+\sum_{r+s=n}(-1)^{l} \operatorname{dim}\left(\operatorname{Im} d_{r, s}^{i}\right)
\end{aligned}
$$


As $l$ goes to infinity, this incorporates the extra term into the limit and so $\chi\left(E_{*, *}^{i}\right)=\chi\left(E_{*, *}^{i+1}\right)$. This proves the relation between the Euler characteristics.

As a final application, we see the spectral sequence of a fibration related to the classical groups:

Example 1.2.11: We have a natural fibration $S U(2) \hookrightarrow S U(3) \rightarrow S U(3) / S U(2)$ with natural identifications $S U(2) \approx S^{3}$ and $S U(3) / S U(2) \approx S^{5}$. This fibration satisfies the hypothesis of Theorem 1.2 .3 and, therefore, we obtain a spectral sequence with $E^{2}$ page of the following form:

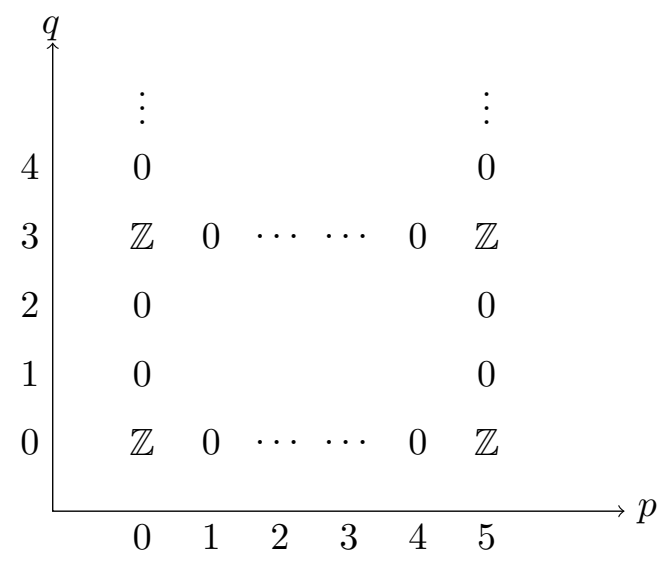

We see that, by the definition of the differentials, all of $d^{r}$ will be zero (no arrows connect nontrivial groups). Therefore, all groups on the second page remain until $E^{\infty}$, which implies

$$
H_{k}(S U(3))=\left\{\begin{array}{lc}
\mathbb{Z}, & k=0,3,5,8 \\
0, & \text { otherwise }
\end{array}\right.
$$

Using Hurewicz Theorem, we see that $\pi_{0}(S U(3))=\pi_{1}(S U(3))=\pi_{2}(S U(3))=0$ and $\pi_{3}(S U(3))=$ $\mathbb{Z}$.

Now that we have developed some examples, we dedicate the rest of this section to prove Theorem 1.2 .3 .

When $B$ is a CW-complex, we have already discussed which filtration to use on $X$ and why this sequence satisfies the hypothesis of Theorem 1.2 .2 . Therefore, we need to prove that the second page of the spectral sequence satisfies $E_{p, q}^{2}=H_{p}\left(B ; H_{q}(F ; G)\right)$.

We know that each term $E_{p, q}^{2}$ is the homology of the respective chain complex of $E_{p, q}^{1}=$ $H_{p+q}\left(X_{p}, X_{p-1} ; G\right)$ with differential $d^{1}$. What we will do is show that such a complex isomorphic to the cellular chain complex of $B$ with coefficients in $H_{q}(F ; G)$. Precisely, we will create an isomorphism $\psi$ of chain complexes:

$$
\begin{gathered}
\cdots \longrightarrow H_{p+q}\left(X_{p}, X_{p-1} ; G\right) \longrightarrow{ }^{d^{1}} \longrightarrow \\
\psi \downarrow \\
\downarrow \\
\cdots \longrightarrow H_{p+q-1}\left(X_{p-1}, X_{p-2} ; G\right) \longrightarrow \\
\psi \downarrow \\
\cdots H_{p}\left(B^{p}, B^{p-1} ; \mathbb{Z}\right) \otimes H_{q}(F ; G) \stackrel{\partial \otimes I d}{\longrightarrow} H_{p-1}\left(B^{p-1}, B^{p-2} ; \mathbb{Z}\right) \otimes H_{q}(F ; G) \longrightarrow \cdots
\end{gathered}
$$

We can think intuitively of this isomorphism the following way: similar to seeing the group $H_{p}\left(B^{p}, B^{p-1} ; \mathbb{Z}\right)$ as generated by the $p$-cells of $B$, we can think of $H_{p+q}\left(X_{p}, X_{p-1} ; G\right)$ as being 
somehow generated by the preimages of such $p$-cells on the fibration. One of the ways to see this is using an argument similar to the one used to prove that $H_{p}\left(B^{p}, B^{p-1} ; \mathbb{Z}\right)$ is generated by the $p$-cells of $B$ : we know there is a map

$$
\phi: \coprod_{\alpha}\left(D_{\alpha}^{p}, S_{\alpha}^{p-1}\right) \rightarrow\left(B^{p}, B^{p-1}\right)
$$

which, in each $D_{\alpha}$, is the attaching map of the cell $e_{\alpha}^{p}$. Notice that, through this definition, $\phi$ is a homeomorphism on the interior of $D_{\alpha}^{p}$.

We know that $B^{p-1}$ is a deformation retract of a neighbourhood $N$ in $B^{p}$, so we have (through the long exact sequence of the triple $\left.\left(B^{p-1}, N, B^{p}\right)\right)$ that $H_{p}\left(B^{p}, B^{p-1}\right) \approx H_{p}\left(B^{p}, N\right)$, which is, by excision, isomorphic to $H_{p}\left(B^{p}-B^{p-1}, N-B^{p-1}\right)$. Since the restriction of $\phi$ is a homeomorphism onto $B^{p}-B^{p-1}$, we get

$$
\begin{aligned}
H_{p}\left(B^{p}, B^{p-1}\right) & \approx H_{p}\left(B^{p}-B^{p-1}, N-B^{p-1}\right) \\
& \approx H_{p}\left(\coprod^{\alpha} \stackrel{\circ}{\alpha}_{\alpha}^{p}, \coprod^{\alpha}\left(\stackrel{\circ}{D}_{\alpha}^{p} \cap \phi^{-1}(N)\right)\right) \\
& \approx \bigoplus_{\alpha} H_{p}\left(D_{\alpha}^{p}, S_{\alpha}^{p-1}\right)
\end{aligned}
$$

where the last isomorphism can be obtained using excision and the exact sequence of the pair, just as before.

Since $H_{p}\left(D_{\alpha}^{p}, S_{\alpha}^{p-1}\right)$ is the $p$-th homology group of a sphere $S^{p}$, we get that $H_{p}\left(B^{p}, B^{p-1}\right)$ has indeed one generator for each $p$-cell.

The reasoning above can be almost completely repeated when we look at the preimages of the $p$-cells in $X^{p}$. The main difference is that, now, we can't say that the $p$-th relative homology group of the preimage is somehow related to the $p$ sphere, but we can see that $H_{p+q}\left(X_{p}, X_{p-1}\right)$ can be written as a direct sum of the $(p+q)$-relative homology groups of the preimages of the cells.

Intuitively, the preimage of a disk $D^{p}$ can be seen as a family $\left\{D_{f}^{p}\right\}_{f}$ indexed by the elements of $F$. With this intuitive idea, what we show is that the relative $(p+q)$ homology group of such preimages with coefficients in $G$ is isomorphic to $H_{q}(F ; G)$, and this gives the desired result.

Lemma 1.2.5: If $F \rightarrow X \rightarrow B$ is a filtration satisfying the hypothesis of Theorem 1.2.3 and $B$ is a $C W$-complex, there exists an isomorphism $\psi$ between the groups $E_{p, q}^{1}$ and $H_{p}\left(B^{p}, B^{p-1} ; \mathbb{Z}\right) \otimes$ $H_{q}(F ; G)$.

Proof of Lemma 1.2.5: We know that $H_{p}\left(B^{p}, B^{p-1} ; \mathbb{Z}\right)$ is the free abelian group generated by the $p$-cells of $B$. Therefore, $H_{p}\left(B^{p}, B^{p-1} ; \mathbb{Z}\right) \otimes H_{q}(F ; G)$ is isomorphic to the direct sum of $H_{q}(F ; G)$, one for each $p$-cell, $e_{\alpha}^{p}$, of $B: H_{p}\left(B^{p}, B^{p-1} ; \mathbb{Z}\right) \otimes H_{q}(F ; G) \simeq \bigoplus_{\alpha} H_{q}(F ; G)$.

Now we write $H_{p+q}\left(X_{p}, X_{p-1} ; G\right)$ in terms of the relative homology groups of preimages of $p$ -cells: for each $p$-cell $e_{\alpha}^{p}$ in $B$, let $\phi_{\alpha}: D_{\alpha}^{p} \rightarrow B^{p}$ be its characteristic map. Let $\tilde{D}_{\alpha}^{p}=\phi_{\alpha}^{*}\left(X_{p}\right)$, be the pullback fibration over $D_{\alpha}^{p}$, that is:

$$
\tilde{D}_{\alpha}^{p}=\left\{(x, d): x \in X^{p} \text { and } d \in D_{\alpha}^{p} \text {, such that } \pi(x)=\phi_{\alpha}(d)\right\}
$$


and let $\tilde{S}_{\alpha}^{p-1}$ be the part of $\tilde{D}_{\alpha}^{p}$ over $S_{\alpha}^{p-1}$. Doing this for each $p$-cell of $B$, we get a map

$$
\begin{aligned}
\tilde{\phi}: \coprod_{\alpha}\left(\tilde{D}_{\alpha}^{p}, \tilde{S}_{\alpha}^{p-1}\right) & \rightarrow\left(X_{p}, X_{p-1}\right) \\
(x, d) & \rightarrow x
\end{aligned}
$$

which sends the component $\tilde{D}_{\alpha}^{p}$ precisely to the preimage of the cell $e_{\alpha}^{p}$.

Since $B^{p-1}$ is a deformation retract of a neighbourhood $N$ in $B^{p}$, the homotopy lifting property shows that $X_{p-1}$ is a weak deformation retract of $\pi^{-1}(N)$ in $X_{p}$; weak because it cannot be said that the points in $X_{p-1}$ are fixed for all $t \in[0,1]$. However, the image of $X_{p-1}$ is in $X_{p-1}$ during all the homotopy, and therefore, we see that it establishes a homotopy equivalence between $\pi^{-1}(N)$ and $X_{p-1}$. Then, for all $k>0, H_{k}\left(X_{p}, X_{p-1} ; G\right) \approx H_{k}\left(X_{p}, \pi^{-1}(N) ; G\right)$ which is, by excision, isomorphic to

$$
\begin{aligned}
H_{k}\left(X_{p}-X_{p-1}, \pi^{-1}(N)-X_{p-1} ; G\right) & \approx H_{k}\left(\coprod_{\alpha} \pi^{-1}\left(e_{\alpha}^{p}\right), \coprod_{\alpha} \pi^{-1}\left(N \cap e_{\alpha}^{p}\right) ; G\right) \\
& \approx \bigoplus_{\alpha} H_{k}\left(\pi^{-1}\left(e_{\alpha}^{p}\right), \pi^{-1}\left(N \cap e_{\alpha}^{p}\right) ; G\right)
\end{aligned}
$$

Since the restriction of each $\phi_{\alpha}$ to $D_{\alpha}^{p}-S_{\alpha}^{p-1}$ is a homeomorphism onto its image, we see that $\tilde{\phi}$ is a homeomorphism from $\coprod_{\alpha} \tilde{D}_{\alpha}^{p}-\tilde{S}_{\alpha}^{p-1}$ onto $X^{p}-X^{p-1}$. Then, $H_{k}\left(\pi^{-1}\left(e_{\alpha}^{p}\right), \pi^{-1}\left(N \cap e_{\alpha}^{p}\right) ; G\right)$ is isomorphic to $k$-th relative homology group of $\left(\tilde{D}_{\alpha}^{p}-\tilde{S}_{\alpha}^{p-1}, V-\tilde{S}_{\alpha}^{p-1}\right)$, where $V$ is a neighbourhood of $\tilde{S}_{\alpha}^{p-1}$ which is homotopy equivalent to it. By the same reasoning above, we see that $\tilde{\phi}$ induces the isomorphism $\tilde{\phi}_{*}$ in the diagram below:

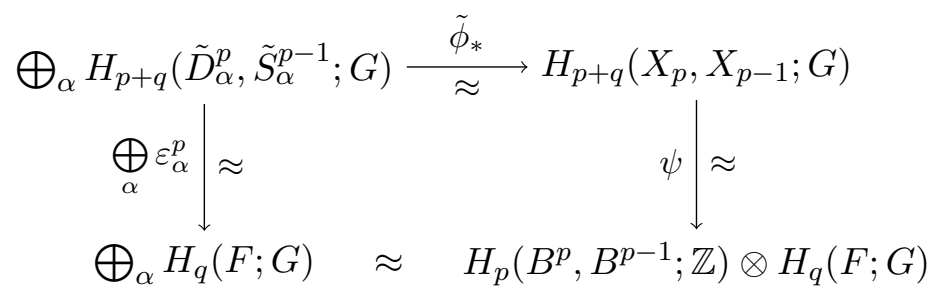

We now construct the left-hand vertical isomorphism by showing that $H_{p+q}\left(\tilde{D}_{\alpha}^{p}, \tilde{S}_{\alpha}^{p-1} ; G\right)$ is isomorphic to $q$-th homology group of a fiber $F_{\alpha}$ of $\pi$. Since $\pi_{1}(B)$ acts trivially on $H_{*}(F ; G)$, we know that all curves $\gamma$ joining the basepoint of $B$ and $\pi\left(F_{\alpha}\right)$ induce the same isomorphism in homology group of the fibers. Therefore, we have a natural isomorphism $H_{q}\left(F_{\alpha} ; G\right) \approx H_{q}(F ; G)$.

To define such $F_{\alpha}$, we make a digression and consider an arbitrary fibration $\tilde{D}^{p} \rightarrow D^{p}$. We partition the boundary sphere $S^{p-1}$ of $D^{p}$ into hemispheres $D_{ \pm}^{p-1}$ intersecting in an equatorial $S^{p-2}$. Iterating this decomposition and letting tildes denote the subspaces of $\tilde{D}^{p}$ lying over these subspaces of $D^{p}$, we look at the following diagram (where we omit the coefficients in $G$ ):

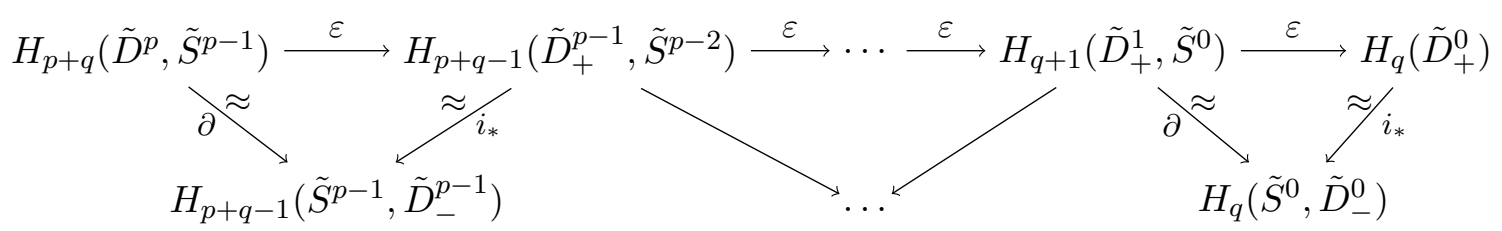


The first boundary map $\partial$ is the one obtained from the long exact sequence of the triple $\left(\tilde{D}^{p}, \tilde{S}^{p-1}, \tilde{D}_{-}^{p-1}\right)$ :

$$
\cdots \rightarrow H_{p+q}\left(\tilde{D}^{p}, \tilde{D}_{-}^{p-1}\right) \rightarrow H_{p+q}\left(\tilde{D}^{p}, \tilde{S}^{p-1}\right) \stackrel{\partial}{\rightarrow} H_{p+q-1}\left(\tilde{S}^{p-1}, \tilde{D}_{-}^{p-1}\right) \rightarrow H_{p+q-1}\left(\tilde{D}^{p}, \tilde{D}_{-}^{p-1}\right) \rightarrow \ldots
$$

using the fact that $\tilde{D}^{p}$ deformation retracts to $\tilde{D}_{-}^{p-1}$, lifting the corresponding deformation retraction of $D^{p}$ to $D_{-}^{p-1}$. We see that both first and last terms on the sequence above are trivial, and therefore $\partial$ is indeed an isomorphism.

The isomorphism $i_{*}$ is the one obtained from excision, considering the spaces

$$
\left(\tilde{D}_{-}^{p-1}-\tilde{S}^{p-2}\right) \subset \tilde{D}_{-}^{p-1} \subset \tilde{S}^{p-1}
$$

Then, using excision, we get an isomorphism $H_{k}\left(\tilde{D}_{+}^{p-1}, \tilde{S}^{p-2}\right) \stackrel{i_{*}}{\rightarrow} H_{k}\left(\tilde{S}^{p-1}, \tilde{D}_{-}^{p-1}\right)$. Combining these isomorphisms $\partial$ and $i_{*}$, we construct $\varepsilon$, which produces an isomorphism $H_{p+q}\left(\tilde{D}^{p}, \tilde{S}^{p-1}\right) \rightarrow$ $H_{q}\left(\tilde{D_{+}^{0}}\right)$.

For each $\alpha$, if we take $\tilde{D}^{p}$ to be $\tilde{D}_{\alpha}^{p}$, we can see that $\tilde{D}_{\alpha+}^{0}$ is precisely the fiber $F_{\alpha}$ of $\pi$ over the point $\phi_{\alpha}\left(D_{\alpha+}^{0}\right)$. Hence, we obtain a natural isomorphism $H_{p+q}\left(\tilde{D}_{\alpha}^{p}, \tilde{S}_{\alpha}^{p-1}\right) \rightarrow H_{q}\left(F_{\alpha} ; G\right) \approx H_{q}(F ; G)$, as required.

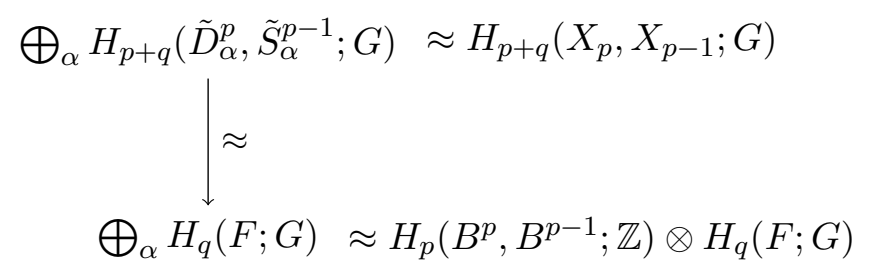

Now we have exhibited all the isomorphisms in the diagram above, we have defined $\psi$ and know that it is a group isomorphism between $E_{p, q}^{1}$ and $H_{p}\left(B^{p}, B^{p-1} ; \mathbb{Z}\right) \otimes H_{q}(F: G)$.

What remains is to show that such map $\psi$ is in fact an isomorphisms of chain complexes. Since we are strongly using the cellular structure of $B$ and its cellular chain complex, it will also be useful to look at the differential map $\partial$ through the degree.

We know that, for each $p$-cell, $e_{\alpha}^{p}$, we have $\partial\left(e_{\alpha}^{p}\right)=\sum \lambda_{\alpha \beta} e_{\beta}^{p-1}$ where each $\lambda_{\alpha \beta}$ is the degree of the maps $S_{\alpha}^{p-1} \rightarrow S_{\beta}^{p-1}$. This is obtained by composing the attaching map $\phi_{\alpha}$ of the cell $e_{\alpha}^{p}$ with the quotient maps $B^{p-1} \rightarrow B^{p-1} / B^{p-2} \rightarrow S_{\beta}^{p-1}$, where the latter is the map that collapses all $(p-1)$-cells except $e_{\beta}^{p-1}$ to a point, and the resulting sphere is identified with $S_{\beta}^{p-1}$ using the characteristic map for $e_{\beta}^{p-1}$.

Recall that given a map $f: S^{n} \rightarrow S^{n}$ such that, for some point $y \in S^{n}$, the preimage $f^{-1}(y)$ consists of only finitely many points, say $x_{1}, \ldots, x_{m}$, then the degree of $f$ is the sum of its local degrees, denoted $\left.\operatorname{deg} f\right|_{x_{1}}$. These local values are obtained by looking at disjoint neighbourhoods $U_{1}, \ldots, U_{m}$ of the points $x_{1}, \ldots, x_{m}$ which are mapped into a neighbourhood $V$ of $y$. We see there are isomorphisms $H_{n}\left(U_{i}, U_{i}-x_{i}\right) \approx H_{n}\left(S^{n}\right)$ and $H_{n}(V, V-y) \approx H_{n}\left(S^{n}\right)$ and therefore, the map $f_{*}: H_{n}\left(U_{i}, U_{i}-x_{i}\right) \rightarrow H_{n}(V, V-y)$ is seen as the multiplication by an integer called local degree. 
Since $S^{n}$ is a compact connected manifold, Proposition $\mathbf{1 7 . 8}$ on BT82 tells us that any continuous map $f: S^{n} \rightarrow S^{n}$ is homotopic to a smooth map, $g$, which implies that $\operatorname{deg} f=$ deg g. From Sard's Theorem (Theorem 4.11 on [BT82]), we know $g$ has a regular value $y$, and therefore, $g$ is a local diffeomorphism around $y$. Since $S^{n}$ is compact, we know that the preimage of $y$ consists of finitely many points, and therefore, we can compute its degree of $g$, and consequently $\operatorname{deg} f$, through the local degrees.

We know that homotopic maps induce the same applications in homology. Therefore, we use convenient homotopies of the attaching map of cells to show that $\psi$ commutes with the differentials of the chain complexes.

Lemma 1.2.6: If $F \rightarrow X \rightarrow B$ is a filtration satisfying the hypothesis of Theorem 1.2.3 and $B$ is a $C W$-complex, then the map $\psi$ constructed above is an isomorphism of chain complexes.

Proof of Lemma 1.2.6: What is left to prove is that $(\partial \otimes I d) \psi=\psi d^{1}$.

We know that $E_{p, q}^{1}$ is isomorphic to the direct sum $\bigoplus_{\alpha} H_{p+q}\left(\tilde{D}_{\alpha}^{p}, \tilde{S}_{\alpha}^{p-1} ; G\right)$ with indices on the $p$-cells, $e_{\alpha}^{p}$, of $B$. To understand the compositions above is to understand how they behave on each component of $H_{p+q}\left(\tilde{D}_{\alpha}^{p}, \tilde{S}_{\alpha}^{p-1} ; G\right)$. We also know how $(\partial \otimes I d) \psi$ works in each of them, from the definition:

$$
\begin{aligned}
H_{p+q}\left(\tilde{D}_{\alpha}^{p}, \tilde{S}_{\alpha}^{p-1} ; G\right) & \\
\psi \circ \tilde{\phi}_{\alpha *} \mid & \\
{\left[e_{\alpha}^{p}\right] \otimes H_{q}(F ; G) \longrightarrow } & \left.\partial \sum \lambda_{\alpha \beta} e_{\beta}^{p-1}\right] \otimes H_{q}(F ; G) \\
\text { in } H_{p}\left(B^{p}, B^{p-1} ; \mathbb{Z}\right) \otimes H_{q}(F ; G) & \text { in } H_{p-1}\left(B^{p-1}, B^{p-2} ; \mathbb{Z}\right) \otimes H_{q}(F ; G)
\end{aligned}
$$

Where each $\lambda_{\alpha \beta}$ is the degree of the maps $S_{\alpha}^{p-1} \rightarrow S_{\beta}^{p-1}$ obtained by composing the attaching map $\phi_{\alpha}$ of the cell $e_{\alpha}^{p}$ with the quotient maps $B^{p-1} \rightarrow B^{p-1} / B^{p-2} \rightarrow S_{\beta}^{p-1}$.

We show that, if instead of $(\partial \otimes I d) \psi$ we do $\psi d^{1}$, we obtain the same sum, with coefficients being the degrees $\lambda_{\alpha \beta}$. To do that, we look at $d^{1} \tilde{\phi}_{\alpha *}$ in $H_{p+q-1}\left(X_{p-1}, X_{p-2} ; G\right) \approx$ $\bigoplus_{\beta} H_{p+q-1}\left(\tilde{D}_{\beta}^{p-1}, \tilde{S}_{\beta}^{p-2} ; G\right)$, the latter sum with indices on the $(p-1)$-cells, $e_{\beta}^{p-1}$.

We know that, on the summand $H_{p+q}\left(\tilde{D}_{\alpha}^{p}, \tilde{S}_{\alpha}^{p-1} ; G\right)$ the differential $d^{1}$ is the composition through the lower left corner in the following commutative diagram with coefficients in $G$ ommited:

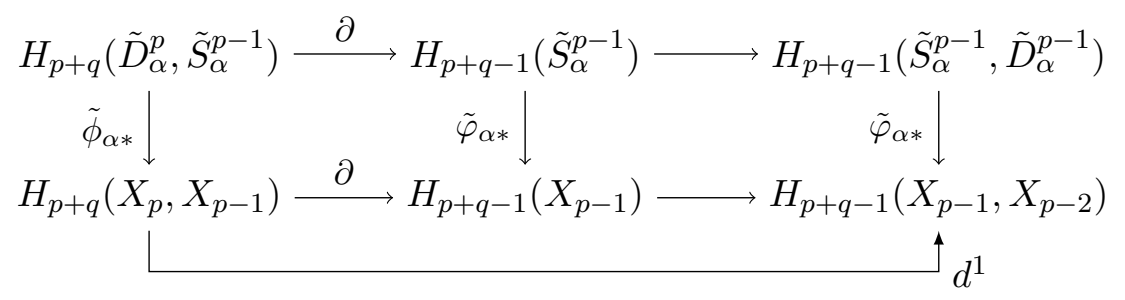

Recall that $\tilde{\phi}_{\alpha *}$ and $\tilde{\varphi}_{\alpha *}$ are induced by the characteristic and attaching map of $e_{\alpha}^{p}$, respectively. We know that the left-hand square commutes and we can look at the composition by using the 
middle vertical map. However, it is more convenient if we define the right-hand vertical map in a way that makes the diagram commute. It is simple to see that $\varphi_{\alpha}$ can be homotoped to a map that sends a hemisphere $D_{\alpha}^{p-1}$ to $X_{p-2}$. Through the homotopy lifting property, we can lift this to a homototpy of $\tilde{\varphi}_{\alpha}$ and so the right-hand map is defined.

We show what this map is by understanding how it behaves on each $H_{p+q-1}\left(\tilde{D}_{\beta}^{p-1}, \tilde{S}_{\beta}^{p-2}\right)$. For the diagram to commute, what we want is that, when we apply $\psi$ on the image of $H_{p+q}\left(\tilde{D}_{\alpha}^{p}, \tilde{S}_{\alpha}^{p-1}\right)$ in $H_{p+q-1}\left(\tilde{D}_{\beta}^{p-1}, \tilde{S}_{\beta}^{p-2}\right)$ we obtain precisely $\lambda_{\alpha \beta} e_{\beta}^{p-1}$. In order to show that this indeed happens, we use the idea of computing the degree of a map through the sum of the local degrees.

Using the ideas discussed before, we know that we can homotope $\varphi_{\alpha}$ to a smooth function and that the degree $\lambda_{\alpha \beta}$ can be computed through the local degree by looking at a regular value in $e_{\beta}^{p-1}$. Using Theorem 4.10 on [Hat02], we can homotope $\varphi_{\alpha}$ once again so that it restricts to homeomorphisms from the interiors of a finite collection of disjoint disks $D_{i}^{p-1}$ (neighbourhoods of the preimages of the regular value) in the hemisphere $D_{\alpha}^{p-1}$ onto $e_{\beta}^{p-1}$ and sends the rest of $D_{\alpha}^{p-1}$ to the complement of $e_{\beta}^{p-1}$ in $B^{p-1}$.

We now use another commutative diagram whose left-hand map is equivalent to the right-hand map on the previous diagram:

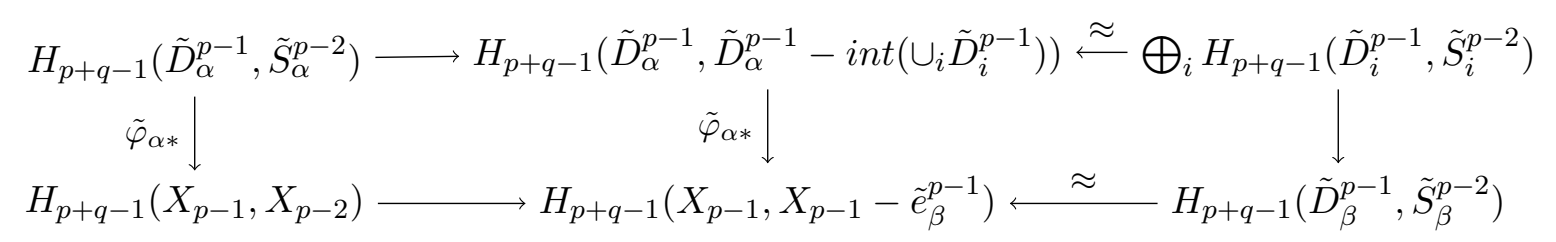

In the process of constructing $\psi$, we constructed isomorphisms between some of the groups in the diagram above and $H_{q}(F, G)$. Precisely, we saw that all $H_{p+q-1}\left(\tilde{D}_{\alpha}^{p-1}, \tilde{S}_{\alpha}^{p-2}\right), H_{p+q-1}\left(\tilde{D}_{i}^{p-1}, \tilde{S}_{i}^{p-2}\right)$ and $H_{p+q-1}\left(\tilde{D}_{\beta}^{p-1}, \tilde{S}_{\beta}^{p-2}\right)$ are isomorphic to $H_{q}(F ; G)$. Through this identification, we see that the map across the top of the diagram becomes the diagonal map $(x) \rightarrow(x, \ldots, x)$. It therefore suffices to show that the right-hand vertical map, when restricted to the $H_{q}(F ; G)$ summand corresponding to $d^{i}$, is $I d$ or $-I d$ according to whether the degree of $\varphi_{\alpha}$ on $d^{i}$ is 1 or -1 .

We see that, for each $i$, we have two fibrations $\tilde{D}_{i}^{p-1} \rightarrow D_{i}^{p-1}$ and $\tilde{D}_{\beta}^{p-1} \rightarrow D_{\beta}^{p-1}$ and a map $\tilde{\varphi}_{\alpha}$ lifting the homeomorphism $\varphi_{i}: D_{i}^{p-1} \rightarrow D_{\beta}^{p-1}$, which is the restriction of $\varphi_{\alpha}$. If the degree of $\varphi_{i}$ is 1 , we may homotope it as a map of pairs $\left(D_{i}^{p-1}, S_{i}^{p-2}\right) \rightarrow\left(D_{\beta}^{p-1}, S_{\beta}^{p-2}\right)$ to be the identity map, and lift this to a homotopy of $\tilde{\varphi}$. Thus, the naturality of the maps we constructed on the previous lemma gives the desired result.

If the degree of $\varphi_{i}$ is -1 , we may assume it is a reflection. Namely, the reflection interchanging $D_{+}^{0}$ and $D_{-}^{0}$ and taking every other $D_{ \pm}^{i}$ to itself. Naturality gives a reduction to the case $k=1$, with $\phi_{i}$ a reflection of $D^{1}$. Using naturality, we can restate what we want by reparametrizing $D^{1}$ by the reflection interchanging its two ends. Now, the short exact sequence of the pair $\left(\tilde{D}^{1}, \tilde{S}^{0}\right)$ breaks into short exact sequences

$$
\left.0 \rightarrow H_{q+1}\left(\tilde{D}^{1}, \tilde{S}^{0}\right) ; G\right) \stackrel{\partial}{\rightarrow} H_{q}\left(\tilde{S}^{0} ; G\right) \stackrel{i_{*}}{\rightarrow} H_{q}\left(\tilde{D}^{1} ; G\right) \rightarrow 0
$$

Since the inclusions $\tilde{D}_{ \pm}^{0} \hookrightarrow D^{1}$ are homotopy equivalences, inducing isomorphisms on homology, 
we think of $H_{q}\left(\tilde{S}^{0} ; G\right)$ as the direct sum of two copies of the same group. Hence the kernel of $i_{*}$ will consist of the pairs $(x,-x)$ in this direct sum. Switching the roles of $D_{+}^{0}$ and $D_{-}^{0}$ in the definition of $\varepsilon$, changes the sign of $\varepsilon$, as required.

This proves our result, because the image of $H_{p+q}\left(\tilde{D}_{\alpha}^{p}, \tilde{S}_{\alpha}^{p-1}\right)$ on the summand $H_{q}(F ; G)$ of $H_{p+q-1}\left(X_{p-1}, X_{p-2}\right)$ corresponding to each cell $e_{\beta}^{p-1}$ is precisely the sum of such local degrees which is $\lambda_{\alpha \beta}$. Therefore $\psi d^{1}\left(H_{p+q-1}\left(\tilde{D}_{\alpha}^{p}, \tilde{S}_{\alpha}^{p-1}\right)\right)=\left(\sum \lambda_{\alpha \beta} e_{\beta}^{p-1}\right)$, as required.

The lemmas we have just proven are enough to conclude the result of Theorem 1.2.3 for when $B$ is a $C W$-complex. We now deduce the results for all $B$, by reducing it to the previous case. We do so by using a CW-approximation.

Definition 1.2.6: Given a space $X$, a weak homotopy equivalence $f: Z \rightarrow X$, where $Z$ is a $C W$-complex, is called a $C W$ approximation to $X$.

Proposition 1.2.7: $\quad$ Every space $X$ has a $C W$ approximation $f: Z \rightarrow X$.

The proof of the result above is found in [Hat02], Proposition 4.13.

If $f: Z \rightarrow X$ is a $\mathrm{CW}$ approximation, we know that $Z$ and $X$ have the same homotopy groups, but we have even more: Proposition 4.1 on Hat02 tells us that if $f: Z \rightarrow X$ is a CW approximation, then $Z$ and $X$ have the same homology groups. We use this relation to finish the proof for when $B$ is not a CW-complex.

Proof of Theorem 1.2.3: The case when $B$ is a CW-complex is completely treated in Lemmas 1.2 .5 and 1.2 .6 .

When $B$ is not a CW-complex, let $f: B^{\prime} \rightarrow B$ be a CW-approximation to $B$, with $\pi^{\prime}: X^{\prime} \rightarrow B^{\prime}$ the pullback to the given fibration $\pi: X \rightarrow B$.

Since $X^{\prime}=\left\{(x, d): x \in X, d \in B^{\prime}\right.$ such that $\left.\pi(x)=f(d)\right\}$, we see that the map $h: X^{\prime} \rightarrow X$, given by $(x, d) \rightarrow x$, gives a homeomorphism between the fiber of each $d \in B^{\prime}$ and $F_{f(d)}$, the fiber of $f(d)$ through $\pi$. Thus, the homotopy exact sequences associated to the fibrations $\pi^{\prime}$ and $\pi$ are related by the maps $f_{\sharp}$ and $h_{\sharp}$ in the following way:

$$
\begin{aligned}
& \cdots \longrightarrow \pi_{n+1}\left(B^{\prime}\right) \longrightarrow \pi_{n}\left(F^{\prime}\right) \longrightarrow \pi_{n}\left(X^{\prime}\right) \longrightarrow \pi_{n}\left(B^{\prime}\right) \longrightarrow \pi_{n-1}\left(F^{\prime}\right) \longrightarrow \cdots \\
& f_{\sharp} \downarrow \approx \quad h_{\sharp} \downarrow \approx \quad h_{\sharp} \downarrow \quad f_{\sharp} \downarrow \approx \quad h_{\sharp} \downarrow \approx \\
& \cdots \longrightarrow \pi_{n+1}(B) \longrightarrow \pi_{n}(F) \longrightarrow \pi_{n}(X) \longrightarrow \pi_{n}(B) \longrightarrow \pi_{n-1}(F) \longrightarrow \cdots
\end{aligned}
$$

By the five lemma, we see that $h$ induces isomorphisms in all homotopy groups of $X$. By Proposition 4.21 on [Hat02], we see that such maps also induce isomorphisms between the homology groups of such spaces with coefficients in an arbitrary group $G$.

Moreover, since the action of $\pi_{1}\left(B^{\prime}\right)$ on $H_{*}(F ; G)$ is the pullback of the action of $\pi_{1}(B)$, we know it is also trivial. Therefore, $\pi^{\prime}: X^{\prime} \rightarrow B^{\prime}$ satisfies the hypothesis of the proven Theorem 1.2.3, and the spectral sequence of $\pi^{\prime}$ gives a spectral sequence for $\pi: X \rightarrow B$. 


\subsection{Eilenberg-MacLane Spaces}

Eilenberg-MacLane Spaces can be thought of as the building blocks of Homotopy Theory.

If we consider Homology Theory of CW-Complexes, we can think that the basic objects are spheres. That is for the simplicity of their homology groups, which are only non-trivial in two dimensions, but also because they are at the centre of the construction of CW-complexes. Unfortunately for Homotopy Theory, spheres are not so well behaved: far from having only one non-trivial homotopy group, to determine all of them remains an unsolved problem.

It is as parallel to the role of spheres in homology that we think of the definition of EilebergMacLane spaces, trying to build objects which play an analogous role in Homotopy Theory.

Precisely, we have

Definition 1.3.1: $\quad$ Let $n$ be a positive integer and $\pi$ a group, abelian if $n>1$. A $C W$-complex, $Y$, is said to be a $K(\pi, n)$ space if

$$
\pi_{k}(Y)= \begin{cases}0 & \text { if } k \neq n \\ \pi & \text { if } k=n\end{cases}
$$

A $K(\pi, n)$ space is called an Eilenberg-MacLane space.

Some of the spaces defined above are natural to Topology, for instance $S^{1}$ is a $K(\mathbb{Z}, 1)$ space, the torus $\mathbb{T}^{2}$ is a $K(\mathbb{Z} \oplus \mathbb{Z}, 1)$ and $\mathbb{C} P^{\infty}$ is a $K(\mathbb{Z}, 2)$ space. However, many such spaces are constructed with sophisticated techniques.

In this section, we address some questions which immediately arise from this definition, related to the existence, uniqueness and generality of such spaces. We end by showing how these spaces relate to computing homotopy groups with the use of spectral sequences.

\subsubsection{Properties and Examples}

A first natural question which arises from the definition of Eilenberg-MacLane spaces is: do those spaces always exist? Precisely, given any $n>0$ and group $\pi$, abelian if $n>1$, is there always a $K(\pi, n)$ space? Fortunately, the answer is positive and this result is proven in a constructive manner using the following well-known results of algebraic topology:

Theorem 1.3.1: (Cellular Approximation Theorem) Any map $f: X \rightarrow Y$ between $C W$ complexes is homotopic to a cellular map. Also, if $f$ is already a cellular map at a subcomplex $A \subset X$, we can choose a homotopy which is stationary at $A$.

Corollary 1.3.2: For all $n$, the inclusion $X^{n} \hookrightarrow X$ induces an isomorphism at $\pi_{i}$, for $i<n$ and a surjection if $i=n$.

For proofs of such results, see Theorem 4.8 and Corollary 4.12 on Hat02]. 
Theorem 1.3.3: Given an integer $n>0$ and a group $\pi$, abelian if $n>1$, then there exists a $C W$-complex which is a $K(\pi, n)$ space.

Proof: We define such $K(\pi, n)$ space by constructing, recursively, its skeleton.

We define the $n$-skeleton of such space in a way to trivialise the homotopy groups in dimensions smaller than $n$, and thinking of the generators of the group $\pi$. We then add $(n+1)$-cells to create the relations of the group $\pi$, and add cells of arbitrarily high dimension to guarantee the trivialisation of the homotopy groups with dimension greater than $n$.

Let $\left\langle g^{i}, i \in I \mid r_{j}, j \in J\right\rangle$ be a representation of the group $\pi$ by generators and relations.

We begin the construction of the space with a wedge sum of $S^{n}$ 's, one for each generator of $\pi$, which will be the $n$-skeleton of the space. Precisely, $Y^{n}=\bigvee_{i \in I} S_{i}^{n}$. By the Cellular Approximation Theorem, 1.3.1), $\pi_{k}\left(Y^{n}\right)=0$, for all $k<n$. If $n=1$, by the Van Kampen Theorem (Theorem 1.20, [Hat02]), $\pi_{1}\left(Y^{1}\right)$ is the free group over the generators of $\pi$; and if $n>1$, by the Hurewicz Theorem (Theorem 1.2.4), $\pi_{n}\left(Y^{n}\right)$ is the free abelian group over the generators of $\pi$.

For each relation $r_{j}$ of $\pi$, we add a $(n+1)$-cell, $e_{j}^{n+1}$ with attaching map defined by $r_{j}$. We then define $Y^{n+1}=Y^{n} \cup\left\{e_{j}^{n+1}\right\}_{J}$. Now

$$
\pi_{k}\left(Y^{n+1}\right)= \begin{cases}0 & \text { if } k<n \\ \pi & \text { if } k=n\end{cases}
$$

The case $n=1$ follows directly from Van Kampen Theorem. If $n>1$, the result also follows from the Cellular Approximation Theorem and the Hurewicz Theorem.

We now add $(n+2)$-cells in such a way as to trivialise the homotopy group in dimension $n+1$. Let $\left\{\left[g_{l}\right]\right\}_{L}$ be a set of generators of $\pi_{n+1}\left(Y^{n+1}\right)$, where $g_{l}: S^{n+1} \rightarrow Y^{n+1}$, for all $l \in L$. For each of the generators of $\pi_{n+1}\left(Y^{n+1}\right)$ we add a $(n+2)$-cell $e_{l}^{n+2}$ with attaching map defined by $g_{l}$, and define $Y^{n+2}=Y^{n+1} \cup\left\{e_{l}^{n+2}\right\}_{L}$. By the Corollary 1.3.2, we know that adding such cells does not affect the homotopy groups in dimensions less or equal to $n$, and also that all maps $S^{n+1} \rightarrow Y^{n+2}$ are null-homotopic in $Y^{n+2}$. Then, we have

$$
\pi_{k}\left(Y^{n+2}\right)= \begin{cases}0, & \text { if } k<n, k=n+1 \\ \pi, & \text { if } k=n\end{cases}
$$

Similar to above, we now define the $(n+3)$-skeleton of $Y$, in such a way as to trivialise the $(n+2)$-homotopý group, adding for each generator $\left[g_{l}\right]$ of $\pi_{n+2}\left(Y^{n+2}\right)$ a $(n+3)$-cell with the attaching map defined by $g_{l}$.

Recursively, we build the $(k+1)$-skeleton of $Y$ in a way as to trivialise the $k$-th homotopy group. The union $Y=\bigcup Y^{k}$ is a $K(\pi, n)$ space, as required.

Corollary 1.3.4: For any sequence of groups $\pi_{0}, \pi_{1}, \pi_{2}, \ldots, \pi_{i}, \ldots$, abelian for $i>1$, there exists a $C W$-complex $X$ such that $\pi_{i}(X)=\pi_{i}$, for all $i \geq 0$.

Proof: It suffices to take 


$$
X=K\left(\pi_{0}, 0\right) \times K\left(\pi_{1}, 1\right) \times K\left(\pi_{2}, 2\right) \times \cdots \times K\left(\pi_{i}, i\right) \times \cdots
$$

We have shown above the existence of arbitrary Eilenberg-MacLane spaces and a way they can be constructed. As a very clear example of such construction, we have $\mathbb{R} P^{\infty}$, which we will study next, and through which we will also introduce another well known manner of building Eilenberg-MacLane space, called telescoping construction.

Example 1.3.1: $\quad$ (The infinite real projective space) The real projective space $\mathbb{R} P^{n}$ can be seen as the quotient of $S^{n}$ under the equivalence relation which identifies antipodal points. We then have a fibration $S^{0} \rightarrow S^{n} \rightarrow \mathbb{R} P^{n}$, which gives rise to a long exact sequence in homotopy:

$$
\cdots \longrightarrow \pi_{q+1}\left(S^{0}\right) \longrightarrow \pi_{q}\left(S^{n}\right) \longrightarrow \pi_{q}\left(\mathbb{R} P^{n}\right) \longrightarrow \pi_{q}\left(S^{0}\right) \longrightarrow \cdots
$$

From the sequence above, we see that, for each $n$, all the $\pi_{i}\left(\mathbb{R} P^{n}\right)$ are trivial if $i<n$ except in dimension one when the group is $\mathbb{Z}_{2}$.

Notice that we can also think of the projective space as the quotient of an $n$ disk with the antipodal points of its boundary $S^{n-1}$ identified. This way, we see that $\mathbb{R} P^{n}$ can be obtained from $\mathbb{R} P^{n-1}$ by attaching an $n$-cell and actually, when it does so, it trivialises the $(n-1)$-th homotopy group. We then get a series of inclusions:

$$
\{\text { point }\} \hookrightarrow \ldots \hookrightarrow \mathbb{R} P^{n} \hookrightarrow \mathbb{R} P^{n+1} \hookrightarrow \ldots
$$

We can think of the infinite real projective space $\mathbb{R} P^{\infty}$ as the limit of such spaces, that is, a space in which each $n$-skeleton would be an $\mathbb{R} P^{n}$ and the $(n+1)$-skeleton is obtained by the previous one by attaching an $(n+1)$-cell. Now, we have seen that, as $n$ increases, the all the groups $\pi_{i}\left(\mathbb{R} P^{n}\right)$ are trivialised for $i<n$ except in dimension one. Therefore, we can suspect that, for $\mathbb{R} P^{\infty}$, all homotopy groups will be zero except in dimension one. Indeed, for $n>1$, the image of any map $f: S^{n} \rightarrow \mathbb{R} P^{\infty}$ is compact in $\mathbb{R} P^{\infty}$, and therefore, is contained in the $p$-th skeleton, for some positive integer $p$. Without loss of generality, we can assume that $p>n$, then since $\pi_{n}\left(X^{p}\right)=\pi_{n}\left(\mathbb{R} P^{p}\right)=0$, we know that $f$ is nullhomotopic in $X^{p}$. Therefore $\pi_{n}\left(\mathbb{R} P^{\infty}\right)=0$, for all $n \neq 1$.

We have another way to see that such property holds using what is called telescoping construction: we glue together, via the natural inclusions, all the finite real projective spaces

$$
\frac{\coprod_{n} \mathbb{R} P^{n} \times I}{(x, 1) \sim(i(x), 0)}
$$

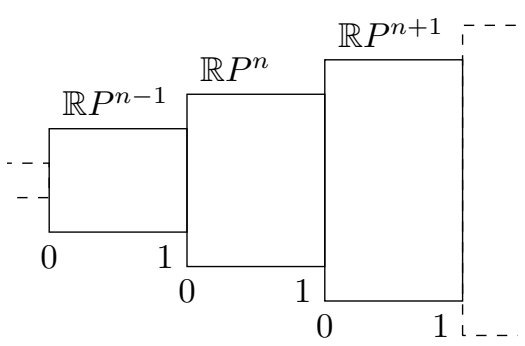

This space is actually homotopy equivalent to $\mathbb{R} P^{\infty}$ as we defined above, and pictorially it can be seen as an infinite telescope, as depicted on the figure above.

Applying the same telescopic construction now to the sequence of spheres (each seen as the equator 
of the next)

$$
\{\text { point }\} \hookrightarrow \ldots \hookrightarrow S^{n} \hookrightarrow S^{n+1} \hookrightarrow \ldots
$$

we obtain the infinite sphere

$$
S^{\infty}=\frac{\coprod_{n} S^{n} \times I}{(x, 1) \sim(i(x), 0)}
$$

which will be a double cover of $\mathbb{R} P^{\infty}$. By the same reasoning on the compacity of images we used above, we can see that $S^{\infty}$ has no homotopy in any dimension. Therefore, from the homotopy exact sequence of the fibration $S^{0} \rightarrow S^{\infty} \rightarrow \mathbb{R} P^{\infty}$, we get that $\mathbb{R} P^{\infty}$ is a $K\left(\mathbb{Z}_{2}, 1\right)$.

Example 1.3.2: $\quad$ (The infinite complex projective space) We can define $\mathbb{C} P^{\infty}$ by gluing together all the $\mathbb{C} P^{n}$ 's, as in the previous example. Then, applying the telescoping construction to the sequences

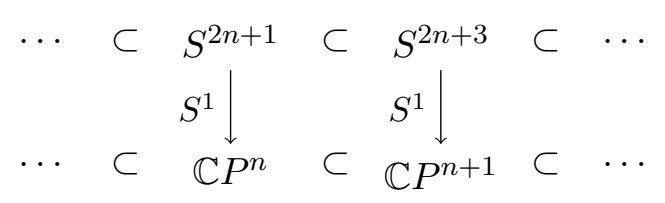

We obtain the fibering $S^{1} \rightarrow S^{\infty} \rightarrow \mathbb{C} P^{\infty}$ and from its homotopy exact sequence, we see that $\mathbb{C} P^{\infty}$ is a $K(\mathbb{Z}, 2)$ space.

Example 1.3.3: $\quad$ (Infinite Lens Spaces) For every positive integers $m$ and $n$, we define the Lens space $L(n, m)$ as the quotient of $S^{2 n+1} \subset \mathbb{C}^{n+1}$ by a free action of $\mathbb{Z}_{m}$ given by

$$
i\left(z_{0}, \ldots, z_{n}\right) \doteq\left(e^{\frac{2 \pi i}{m}} z_{0}, \ldots, e^{\frac{2 \pi i}{m}} z_{n}\right)
$$

We define the $m$-th infinite Lens space $L(\infty, m)$ as the gluing of such $L(n, m)$, applying the telescoping construction to the sequences

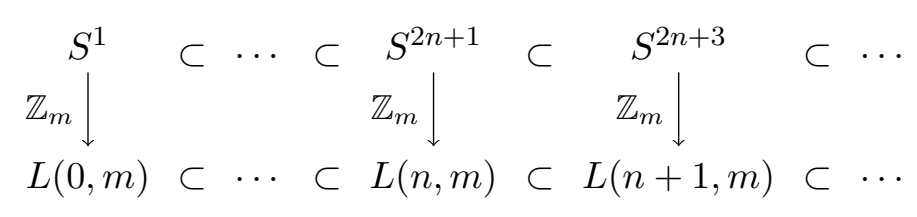

We then obtain the $m$-sheeted covering $\mathbb{Z}_{m} \rightarrow S^{\infty} \rightarrow L(\infty, m)$ and from its homotopy exact sequence, we see that $L(\infty, m)$ is a $K\left(\mathbb{Z}_{m}, 1\right)$ space.

Example 1.3.4: $\quad(K(\mathbb{Q}, 1))$ We will construct this space using an argument similar to the telescoping construction, called mapping telescope. Consider the sphere $S^{1}$ and, for each $n, a$ map $f_{n}: S^{1} \rightarrow S^{1}$ of degree $n$. We define the space

$$
\operatorname{Tel}\left(S^{1}\right)=\frac{\coprod_{n} S^{1} \times[n, n+1]}{\sim}
$$

where $\sim$ identifies $(x, n+1) \in S^{1} \times[n, n+1]$ with $f_{n}(x) \in S^{1} \times[n+1, n+2]$.

Through the same argument of compact images used on the previous examples, we can see that $\pi_{i}\left(\operatorname{Tel}\left(S^{1}\right)\right)=0$, for all $i \geq 2$ and $i=0$. 
We now move to understand the first homotopy group. On one hand, Van-Kampen Theorem can be easily modified to show that the fundamental group preserves colimits (this can be seen in section 2.7 of [May99]). On the other hand, we have the algebraic result that $\mathbb{Q}$ is the filtered colimit $\mathbb{Z} \rightarrow \mathbb{Z} \rightarrow \ldots$ where the successive maps are precisely the multiplications by $1,2, \ldots$ Combining these results, we can see that $\pi_{1}\left(\operatorname{Tel}\left(S^{1}\right)\right)$ is precisely $\mathbb{Q}$.

Although we proved the existence of Eilenberg-MacLane spaces by a constructive method, we can see that such construction involves adding cells of arbitrarily high dimensions. Even the telescoping construction we considered above, depends on such infinite attachments. Therefore, we can see that, although there are natural examples of Eilenberg-MacLane spaces, such as $S^{1}$, there is no guarantee that such spaces are, in some way, simple or natural in Algebraic Topology.

It is natural then to ask whether we could have other, simpler, Eilenberg-MacLane spaces. For instance, if there are $K(\mathbb{Z}, 2)$ 's of finite dimension. It is easy to see that two Eilenberg-MacLane spaces need not be homeomorphic, for instance looking at $S^{1}$ and $R^{2} \backslash\{(0,0)\}$, which are both $K(\mathbb{Z}, 1)$. But actually, such spaces verify a uniqueness property inside Homotopy Theory:

Theorem 1.3.5: Given an integer $n>0$ and a group $\pi$, abelian if $n>1$, any $K(\pi, n)$ spaces are homotopy equivalent.

We will prove this result by showing that all Eilenberg-MacLane spaces will be homotopy equivalent to the one constructed in the proof of Theorem 1.3.3. And this answers the question of whether there could be simpler spaces of such form: not for Homotopy Theory.

We will look at this matter using a very important result on CW-complex, whose proof can be seen in [Hat02], Theorem 4.5:

Theorem 1.3.6: (Whitehead's Theorem) Let $f: X \rightarrow Y$ be a map between connected $C W$ complexes which induces isomorphisms $f_{*}: \pi_{n}(X) \rightarrow \pi_{n}(Y)$, for all $n$. Then $f$ is a homotopy equivalence. Moreover, if $f$ is the inclusion of a subcomplex $X \hookrightarrow Y$, we have that $X$ is a deformation retract of $Y$.

By Whitehead's Theorem (Theorem 1.3.6), if there is a map between two CW-complexes which induces isomorphisms in all homotopy groups, then such application is a homotopy equivalence. When we think of two $K(\pi, n)$ spaces, we know that all homotopy groups are trivial, except in dimension $n$. Then, to verify that a map between such two spaces is a homotopy equivalence is the same as verifying if the homomorphism induced on the $n$th homotopy group is an isomorphism.

We will show that, for each automorphism $\phi$ of $\pi$, there is a map between any two $K(\pi, n)$ spaces, in which the homomorphism induced in the $n$-th homotopy group is $\phi$. With this result, we can obtain a homotopy equivalence between such spaces by choosing a map in which the induced homomorphism is the identity.

We will first construct such map on the $n$-cells of $K(\pi, n)$, guaranteeing that the induced homomorphism will be precisely $\phi$, and then we will extend such function to the entire space $K(\pi, n)$. In order to do this, we will use some elementary results of Obstruction Theory, which deals with the matter of when a map $g: A \rightarrow Y$, defined on a subspace $A \subset X$, can be continually extended 
to $X \rightarrow Y$. One of the ways to approach such problem is to consider the case in which $(X, A)$ is a CW-pair and try to extend the map to the entire space, by extending recursively to each cell from the $C W$-complex.

If we want to extend a map to the $(n+1)$-cells of a given space, a simplifying hypothesis which will be enough to grasp the Eilenberg-MacLane spaces, is to assume the space $Y$ is $n$-simple. We can think of such property as one who frees us from the basepoint, when looking at homotopy groups. We already know that each path $\gamma$, in $Y$, will give us an isomorphism $\beta_{\gamma}$ between the homotopy groups based on its endpoints. However, this isomorphism may not be canonical, since two distinct paths may produce different maps between the $\pi_{n}$ 's. We say a space is $n$-simple if the isomorphism induced by the paths $\gamma$ on the $n$-th homotopy groups depend only on the endpoints of $\gamma$. Therefore, we have canonical isomorphisms between the $\pi_{n}$ 's on distinct basepoints and this can be used to free the $n$-th homotopy group from the basepoint matter: $\pi_{n}\left(Y, y_{0}\right)=\pi_{n}(Y)$ is in one-to-one correspondence with the homotopy classes of unbased maps $S^{n} \rightarrow Y$, denoted $\left[S^{n} ; Y\right]$. A space which is $n$-simple for all $n$ will be called abelian.

We will decide whether the a map $g$ can be extended to an $(n+1)$-cell, by looking at how it behaves on the boundary of such cell. We know that each $(n+1)$-cell has a characteristic map

$$
\phi_{i}:\left(D^{n+1}, S^{n}\right) \rightarrow\left(e_{i}^{n+1}, \partial e_{i}^{n+1}\right) \subset\left(X^{n+1}, X^{n}\right)
$$

with the restriction to $S^{n}$, called the attaching map $, f_{i}=\left.\phi_{i}\right|_{S^{n}}: S^{n} \rightarrow X^{n}$. If we assume the space $Y$ is $n$-simple, we know that any map $S^{n} \rightarrow Y$ can be seen as representing an element of $\pi_{n}(Y)$, and then we can make the following:

Definition 1.3.2: Given $g: X^{n} \rightarrow Y$, we define the obstruction cochain $\theta^{n+1}(g) \in C^{n+1}\left(X, A ; \pi_{n} Y\right)$ by setting

$$
\theta^{n+1}(g)\left(e_{i}^{n+1}\right)=\left[g \circ f_{i}\right]
$$

on a basis element $e_{i}^{n+1}$ and extend it linearly to the homology group.

It is from such $\theta^{n+1}$ that we can decide for each $(n+1)$-cells if it is possible to extend the map $g$.

Lemma 1.3.7: $\quad \theta^{n+1}(g)\left(e^{n+1}\right)=0$ if and only if $g$ can be extended to a map from $X^{n} \cup\left\{e^{n+1}\right\}$ to $Y$.

Proof of Lemma 1.3.7: This result follows directly from the well known fact that a map $h: S^{n} \rightarrow Y$ is homotopically trivial if and only if $h$ extends to a map $D^{n+1} \rightarrow Y$.

Corollary 1.3.8: $\quad \theta^{n+1}(g)=0$ if and only if $g$ can be extended to a map from $X^{n+1}$ to $Y$.

In this dissertation, Lemma 1.3 .8 will be constantly used and it is enough to give us the results we want. However, it is worth knowing that a much more general result holds regarding when a map can or not be extended:

Theorem 1.3.9: $\quad$ The obstruction cochain $\theta^{n+1}(g)$ is a cocycle. If $\theta^{n+1}(g)$ is cohomologous to 
zero, then the restriction of $g$ to the $(n-1)$-skeleton, $X^{n-1}$, can be extended to the $(n+1)$-skeleton, $X^{n+1}$.

The proof of such result and a deeper approach to such theory can be seen in Chapter 7 of [DK01].

So much for this excursion into Obstruction Theory we now return to the matter of proving "unicity" of Eilenberg-MacLane spaces $K(\pi, n)$ by showing that for each automorphism $\phi$ of $\pi$, there is a map between any two $K(\pi, n)$ spaces, in which the homomorphism induced in the $n$-th homotopy group is $\phi$. To prove the Theorem 1.3 .5 we will use the following result:

Lemma 1.3.10: $\quad$ Let $X$ be a $C W$-complex of the form $\left(\bigvee_{\alpha} S_{\alpha}^{n}\right) \bigcup_{\beta} e_{\beta}^{n+1}$, for some $n \geq 1$. Then, for all homomorphism $\phi: \pi_{n}(X) \rightarrow \pi_{n}(Y)$ with $Y$ arc-connected, there is a map $f: X \rightarrow Y$ with $f_{*}=\phi$.

Proof of Lemma 1.3.10: We wish to buid a map $f: X \rightarrow Y$ such that $f_{*}=\phi: \pi_{n}(X) \rightarrow$ $\pi_{n}(Y)$. Firstly, we define $f$ on the 0 -cell, $x_{0}$ (the point in which all the $S_{\alpha}^{n}$ are connected), choosing an arbitrary basepoint $y_{0} \in Y$.

Now we need to define $f$ on the $n$-cells of $X$ in such a way that the desired result holds. For each $\alpha$, let $i_{\alpha}: S^{n} \rightarrow X$ be the inclusion of $S_{\alpha}^{n}$ in $X$, and let $g_{\alpha}: S^{n} \rightarrow Y$ be a representative of the class $\phi\left(\left[i_{\alpha}\right]\right)$. We define then $f \circ i_{\alpha}(x)=g_{\alpha}(x)$.

Hence, for the map $f: X^{n} \rightarrow Y$ constructed so far, we have $f_{*}\left(\left[i_{\alpha}\right]\right)=\phi\left(\left[i_{\alpha}\right]\right)$, for all $\alpha$. Therefore, since such $i_{\alpha}$ 's are generators of $\pi_{n}\left(X^{n}\right)$, we have that $f_{*}([g])=\phi([g])$, for all $g$ : $\left(S^{n}, s_{0}\right) \rightarrow\left(X, x_{0}\right)$.

It remains now to verify whether such $f$ can be extended to the $(n+1)$-skeleton of $X$. Note that, given any $(n+1)$-cell, $e_{\beta}^{n+1}$ with attaching map $\psi_{\beta}$, we know that $\theta^{n+1}(f)\left(e_{\beta}^{n+1}\right)=f_{*}\left(\left[\psi_{\beta}\right]\right)=$ $\phi\left(\left[\psi_{\beta}\right]\right)=0$ since $\psi_{\beta}$ is nullhomotopic in $X$ and $\phi$ is a homomorphism.

Therefore, by Corollary 1.3.8, we know that $f$ can be extended to all $(n+1)$-cells, and therefore, to all of $X$. Also, we know that $f_{*}=\phi$, since the classes $\left[i_{\alpha}\right]$ generate $\pi_{n}\left(X^{n}\right)$ and then, by the Cellular Approximation Theorem, also generate $\pi_{n}(X)$.

With the result above, the Theorem 1.3 .5 follows naturally. We recall the result below:

Theorem 1.3.5: Given an integer $n>0$ and a group $\pi$, abelian if $n>1$, any $K(\pi, n)$ spaces are homotopy equivalent.

Proof of Theorem 1.3.5: To show that any two $K(\pi, n)$ spaces are homotopy equivalent, we will show that any such spaces have the same homotopy type of a specific $K(\pi, n)$ space, $X$. By the transitivity of homotopy equivalence, we will have the desired result.

The space $X$ we will use is, precisely, the one built through the proof of Theorem 1.3.3, that is, a space formed by a bouquet of $S^{n}$ 's in which we add cells of dimension greater than $n$. Note that $X^{n+1}$, the $(n+1)$-skeleton of $X$, is exactly on the hypotheses of Lemma 1.3.10.

Then, given an arbitrary $K(\pi, n)$ space, $Y$, we know by Lemma 1.3 .10 there exists a map $F: X^{n+1} \rightarrow Y$ such that $F_{*}: \pi_{n}\left(X^{n+1}\right) \rightarrow \pi_{n}(Y)$ is precisely the identity map $I d: \pi \rightarrow \pi$. 
To build the homotopy equivalence we need to extend the map $F$ to the entire space $X$.

Since $\pi_{n+1}(Y)=0$, we know that $\theta^{n+2}(F)$ is null, and therefore, by Corollary 1.3.8, the map $F$ can be extended to $X^{n+2}$. Indeed, since $\pi_{i}(Y)=0$, for all $i>n$, this same argument guarantees that the map $F$ can be extended to all the cells, and therefore, to a function $f: X \rightarrow Y$.

By the Cellular Approximation Theorem we also know that $f_{*}=I d$, and therefore, $f$ induces isomorphisms in all homotopy groups. Hence, by Whitehead's Theorem, $f$ is a homotopy equivalence.

Since there's a uniqueness on Eilenberg-MacLane spaces, some of its properties were also characterised. For instance, the homology groups of $K(\pi, n)$ are entirely determined by $\pi$ and $n$. These, which are denoted $H_{i}(\pi, n ; G)$ are called Eilenberg-MacLane groups. From the Hurewicz Theorem, we already know that $H_{i}(K(\pi, n) ; \mathbb{Z})=0$, for $0<i<n$, and $H_{n}(K(\pi, n) ; \mathbb{Z})=\pi$. However, we wish to understand their behaviour in higher dimensions. We could ask if there is an inductive way to compute such groups, or if $K(\pi, n)$ is somehow related to $K(\pi, m)$ in a way which can be used to understand their homology groups.

In fact, one of such relations is obtained from pathspace fibration of $K(\pi, n)$ :

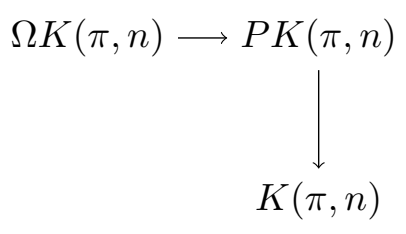

From the homotopy exact sequence of such fibration, we get that, since $P K(\pi, n)$ is contractible,

$$
\pi_{i}(\Omega K(\pi, n)) \simeq \pi_{i+1}(K(\pi, n)) \begin{cases}0 & \text { if } i \neq n-1 \\ \pi & \text { if } i=n-1\end{cases}
$$

Therefore, the loopspace of a $K(\pi, n)$ is a $K(\pi, n-1)$. Notice that, for $n>1$, the base space of the fibration above has trivial fundamental group and, therefore, satisfies the hypothesis of Theorem 1.2.3. Then, using the Spectral Sequence of the Fibration, we get the following:

Theorem 1.3.11: $\quad$ There exists a homology spectral sequence such that its second term $E_{p, q}^{2}$ is isomorphic to $H_{p}\left(K(\pi, n+1) ; H_{q}(\pi, n ; G)\right)$, and its term $E^{\infty}$ is trivial in all dimensions.

The result above shows us it is possible to study Eilenberg-MacLane groups by induction on $n$, starting with the groups $H_{i}(\pi, 1)$, which can be calculated by other methods.

Example 1.3.5: We will show how this method of computation can be used to determine the homology groups of $K(\mathbb{Z}, 2)$, from $K(\mathbb{Z}, 1)=S^{1}$ (since we have already shown that $K(\mathbb{Z}, 2)=$ $\mathbb{C} P^{\infty}$, we could compute the cellular homology directly, however, to do this from the Spectral Sequence of the Fibration gives us an idea of how to discover such groups even when we do not know an explicit example of such a space).

To facilitate notation, we will denote $H_{i}(K(\mathbb{Z}, 2) ; \mathbb{Z})$ by $H_{i}(K)$. By Theorem 1.3 .11 there exists a Spectral Sequence with trivial $E^{\infty}$ in all dimensions and second page of the form 


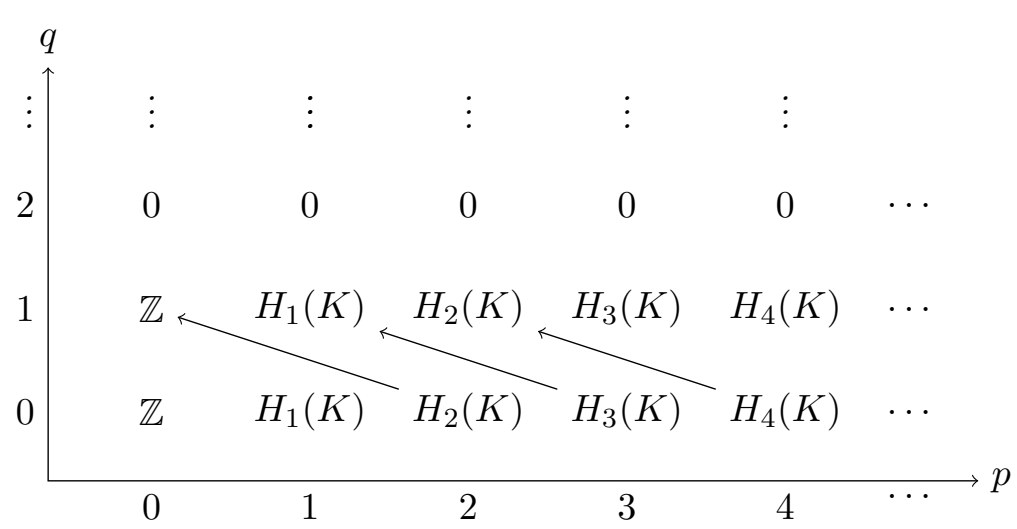

Since the rows with $q>1$ are all trivial and all groups must vanish, we know that all the arrows on page two must be isomorphisms. Therefore, for all positive integers $i$,

$$
H_{2 i}(K(\mathbb{Z}, 2)) \simeq H_{0}(K(\mathbb{Z}, 2))=\mathbb{Z} \quad \text { and } \quad H_{2 i+1}(K(\mathbb{Z}, 2)) \simeq H_{1}(K(\mathbb{Z}, 2))=0
$$

Although the method above is not able to give us all information on Eilenberg-MacLane groups (because, in most cases, we do not have such a well-behaved second page), it is still a very powerful tool to understand such groups.

In the next section, we will show a way to relate arbitrary connected CW-complexes with Eilenberg-MacLane spaces via fibrations.

\subsubsection{Postnikov Towers}

We now aim to establish a parallel among the role of such spaces in Homotopy Theory and the role of spheres in Homology Theory, posing the following question: can any CW-complex be somehow built from a collection of Eilenberg-MacLane spaces?

Indeed, we can think of such spaces as building blocks of CW-complexes, but such construction is not that simple. It isn't true, for instance, that any such space is a product of EilenbergMacLane spaces, but it is true that any connected CW-complex can be thought of as a "twisted product" of those building blocks, from a sequence of fibrations called Postnikov Tower.

Formally, we have the following:

Definition 1.3.3: $\quad$ A Postnikov Tower of a connected space $X$ is a commutative diagram as the one on the right, in which:

1. The inclusion $X \hookrightarrow X_{n}$ induces isomorphisms on the groups $\pi_{i}$, for $i \leq n$;

2. $\pi_{i}\left(X_{n}\right)=0$, for $i>n$.

3. Each map $X_{n} \rightarrow X_{n-1}$ is a fibration, with fiber an Eilenberg-MacLane space.

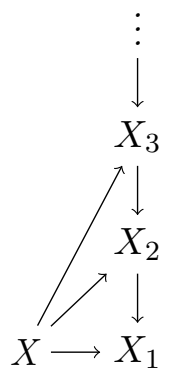

Theorem 1.3.12: $\quad$ Every connected $C W$-complex admits a Postnikov Tower. 
We can build spaces, $Y_{n}$, satisfying the properties 1 and 2 above with the same strategy we used in the proof of Theorem 1.3.3, adding cells of high dimension to trivialise homotopy groups.

Also, with Lemma 1.3.8, it is possible to extend the inclusion $X \hookrightarrow Y_{n-1}$ to a map $Y_{n} \rightarrow Y_{n-1}$, since $\pi_{i}\left(Y_{n-1}\right)=0$, for all $i>n$. However, such maps are not necessarily fibrations.

To guarantee that the property 3 above is satisfied, we will use the results below to transform the maps $Y_{n} \rightarrow Y_{n-1}$ recursively into fibrations. Also, we will see that their fibers are precisely Eilenberg-MaLane spaces.

The next two results show us that any map can be seen as the composition of a homotopy equivalence and a fibration. Therefore, regarding Homotopy Theory, any map can be "transformed" in a fibration.

Preciselly, we have: given any map $f: A \rightarrow B$, we define the space $E_{f}$ as the set of pairs $(a, \gamma)$, such that $a \in A$ and $\gamma: I \rightarrow B$ is a path in $B$ with $\gamma(0)=f(a)$ (such space $E_{f}$ is seen as a subspace of $A \times B^{I}$ with the compact-open topology).

Lemma 1.3.13: $\quad$ The map $p: E_{f} \rightarrow B$ defined by $p(a, \gamma)=\gamma(1)$ is a fibration.

With the result above, we can, given an arbitrary map, get a fibration related to such map. Besides, we get a nice relation between the spaces $E_{f}$ and $A$. Formally:

Lemma 1.3.14: $\quad$ The spaces $A$ and $E_{f}$ are homotopy equivalent.

Indeed, we can think of $A$ as the subspace of $E_{f}$ formed by the pairs $(a, \gamma)$, where $\gamma$ is the constant path $f(a)$. We can then define a deformation retract $H$ from $E_{f}$ to $A \subset E_{f}$, defining $H_{t}(a, \gamma)=\left(a, \gamma_{[0,1-t]}\right)$, where $\gamma_{[0, t]}$ is the path defined by $\gamma(s)$, with $0 \leq s \leq 1-t$.

Therefore, as mentioned above, we can split any map $f: A \rightarrow B$ as the composition of a homotopy equivalence and a fibration.

$$
A \hookrightarrow E_{f} \stackrel{p}{\rightarrow} B
$$

Proof of Theorem 1.3.12: Let $Y_{n}$ be a space constructed the following way: define the $n+1$ skeleton of the space $Y_{n}$ as $X$ and, for each generator $g_{i}$ of $\pi_{n+1}(X)$, we add a $(n+2)$-cell, $e_{i}^{n+2}$ with attaching map $g_{i}$. In order to define the $(n+3)$-skeleton of $Y_{n}$, we add $(n+3)$-cells in such a way to trivialise the generators of $\pi_{n+2}\left(X \cup\left\{e_{i}^{n+2}\right\}\right)$ and so on, as in the proof of Theorem 1.3 .3 .

We already know that such spaces satisfy conditions 1 and 2 required of spaces at the Postnikov Tower of $X$. We wish now to create maps $Y_{n} \rightarrow Y_{n-1}$, which we will later transform in fibrations.

We know there are inclusions $i_{n}: X \hookrightarrow Y_{n}$ and $i_{n-1}: X \hookrightarrow Y_{n-1}$. Then, if we think of $X$ as a subspace of $Y_{n}$, we wish to extend the map $i_{n-1}: X \hookrightarrow Y_{n-1}$ to all $Y_{n}$. However, note that the image of the obstruction cocycle $\theta_{n+2}\left(i_{n-1}\right)$ is contained in $\pi_{n+1}\left(X_{n-1}\right)=0$, and therefore, the obstruction cocycle is null. Therefore, by Lemma 1.3.8, we can extend $i_{n}$ to all the $(n+2)$-cells of $Y_{n}$. And, since $\pi_{i}\left(Y_{n-1}\right)=0$, for all $i>n$, this same argument holds for all other cells of $Y_{n}$, and therefore, the function can be extended to a map $h_{n}: Y_{n} \rightarrow Y_{n-1}$.

Also, note that such map makes the following diagram commute: 


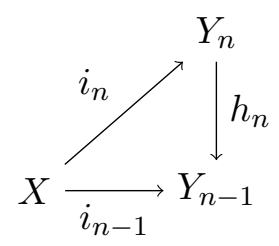

Now, using Lemma 1.3.13, we will build the fibrations recursively. First, let $X_{1}=Y_{1}^{\prime}$ and define $X_{2}=E_{h_{1}}$. By Lemma 1.3.14, we know that $X_{2}$ still satisfies the conditions 1 and 2 of the spaces on the Postnikov Tower and, by Lemma 1.3.13, we know there exists a fibration $p_{1}$ between such spaces (note that this also make the diagram between such spaces commute).

Recursively, having defined $X_{n-1}$, we can think of $Y_{n-1}$ as a subspace of $X_{n-1}$, and then consider $h_{n}: Y_{n} \rightarrow X_{n-1}$ and define $X_{n}=E_{h_{n}}$. And so on.

Then, we get spaces satisfying conditions 1 and 2 and also the fibrations between them. It remains to verify that the fibers $F_{n}$ are Eilenberg-Maclane spaces. From the Long Exact Sequence of the Fibrations, we have

$$
\cdots \rightarrow \pi_{k+1}\left(X_{n-1}\right) \rightarrow \pi_{k}\left(F_{n}\right) \rightarrow \pi_{k}\left(X_{n}\right) \rightarrow \pi_{k}\left(X_{n-1}\right) \rightarrow \cdots
$$

From the relations above, we get the following results:

If $k>n$ we have:

$$
\cdots \rightarrow \underbrace{\pi_{k+1}\left(X_{n-1}\right)}_{0} \rightarrow \pi_{k}\left(F_{n}\right) \rightarrow \underbrace{\pi_{k}\left(X_{n}\right)}_{0} \rightarrow \underbrace{\pi_{k}\left(X_{n-1}\right)}_{0} \rightarrow \cdots
$$

and therefore, $\pi_{k}\left(F_{n}\right)=0$.

If $k=n$ we have:

$$
\cdots \rightarrow \underbrace{\pi_{n+1}\left(X_{n-1}\right)}_{0} \rightarrow \pi_{n}\left(F_{n}\right) \rightarrow \underbrace{\pi_{n}\left(X_{n}\right)}_{\pi_{n}(X)} \rightarrow \underbrace{\pi_{n}\left(X_{n-1}\right)}_{0} \rightarrow \cdots
$$

and therefore, $\pi_{n}\left(F_{n}\right)=\pi_{n}(X)$.

If $k<n$ we have:

$$
\cdots \rightarrow \pi_{k+1}\left(X_{n}\right) \rightarrow \pi_{k+1}\left(X_{n-1}\right) \rightarrow \pi_{k}\left(F_{n}\right) \rightarrow \pi_{k}\left(X_{n}\right) \stackrel{\cong}{\rightarrow} \pi_{k}\left(X_{n-1}\right) \rightarrow \cdots
$$

and therefore, $\pi_{k}\left(F_{n}\right)=0$.

Hence, $F_{n}$ is a $K\left(\pi_{n}(X), n\right)$ space. As required.

With the above result, we have shown that, indeed, Eilenberg-MacLane spaces can be regarded as analogues in Homotopy Theory, to spheres in Homology of CW-complexes. In summary, we have built, for any connected CW-complex, a tower 


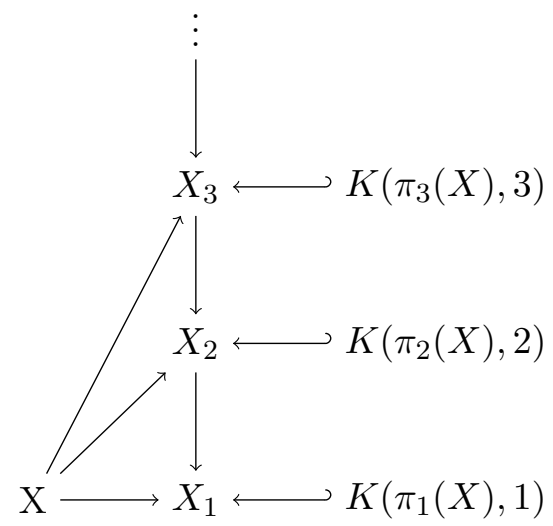

Also, we have the following result:

Lemma 1.3.15: If $\left\{X_{n}^{\prime}\right\}$ satisfies conditions (1) - (3) from the Definition 1.3.3, then $X_{n}^{\prime}$ is homotopy equivalent to the space $Y_{n}$ built in the proof above, for all $n$.

Proof: For all $n$, we know there are inclusions $i_{n}: X \rightarrow Y_{n}$ and $h_{n}: X \rightarrow X_{n}^{\prime}$ which induce isomorphisms in all homotopy groups of dimension less or equal to $n$. We can then think of $X$ as a subspace of $Y_{n}$ and extend the map $h_{n}$ to all $Y_{n}$.

Since $\pi_{n+1}\left(X_{n}^{\prime}\right)=0$, we know that $\theta^{n+2}\left(h_{n}\right)$ is null, and then, by Corollary 1.3.8, $h_{n}$ can be extended to the $(n+2)$-skeleton of $Y_{n}$. Since all homotopy groups of $X_{n}^{\prime}$ of dimension greater than $n$ are trivial, we know that the previous argument guarantees the map $h_{n}$ can be extended to a function $f: Y_{n} \rightarrow X_{n}^{\prime}$. Moreover, we know that $f$ also induces isomorphisms between $\pi_{i}\left(Y_{n}\right)$ and $\pi_{i}\left(X_{n}^{\prime}\right)$, for all $i \leq n$.

Since all other homotopy groups of $Y_{n}$ and $X_{n}^{\prime}$ are trivial, we have that $f$ induces isomorphisms in all homotopy groups. Hence, by Whitehead's Theorem, (Theorem 1.3.6), $f$ is a homotopy equivalence.

With the previous result, we can see that, besides the existence of a Postnikov Tower for any connected $\mathrm{CW}$-complex, it is also true that such construction in unique regarding homotopy.

Now that we have seen how arbitrary connected CW-complexes are related to Eilenberg-MacLane spaces via fibrations, we will see an example of how, using spectral sequences, we can use such tools to compute homotopy groups.

Example 1.3.6: We will deduce $\pi_{4}=\pi_{4}\left(S^{3}\right)$ from the Postnikov Tower of $S^{3}$. In fact, to do so, we will only need to look to a part of such tower, more precisely to the fibration

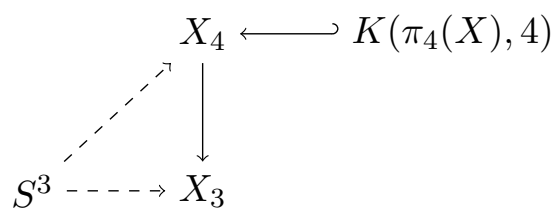

From the definition, we know that $\pi_{i}\left(X_{3}\right)=\pi_{i}\left(S^{3}\right)$, for all $i \leq 3$, and $\pi_{i}\left(X_{3}\right)=0$, otherwise. Then $X_{3}$ is a $K(\mathbb{Z}, 3)$, whose first homology groups, computed in Chapter 3 of [BT82], are given by: 
$\pi_{0}\left(X_{3}\right)=\mathbb{Z} \quad \pi_{1}\left(X_{3}\right)=\pi_{2}\left(X_{3}\right)=0 \quad \pi_{3}\left(X_{3}\right)=\mathbb{Z} \quad \pi_{4}\left(X_{3}\right)=0 \quad \pi_{5}\left(X_{3}\right)=\mathbb{Z}_{2}$

Also, since $\pi_{i}\left(X_{4}\right)=\pi_{i}\left(S^{3}\right)$, for all $i \leq 4$, we know, from the Hurewicz Theorem, that $H_{i}\left(X_{4}\right)=$ 0 , for all $0<i<3$, and $H_{3}\left(X_{4}\right)=\mathbb{Z}$.

Then, we know there is a spectral sequence converging to the homology groups of $X_{4}$, with second page of the form $E_{p, q}^{2}=H_{p}\left(X_{3} ; H_{q}\left(K\left(\pi_{4}, 4\right)\right)\right)$ :

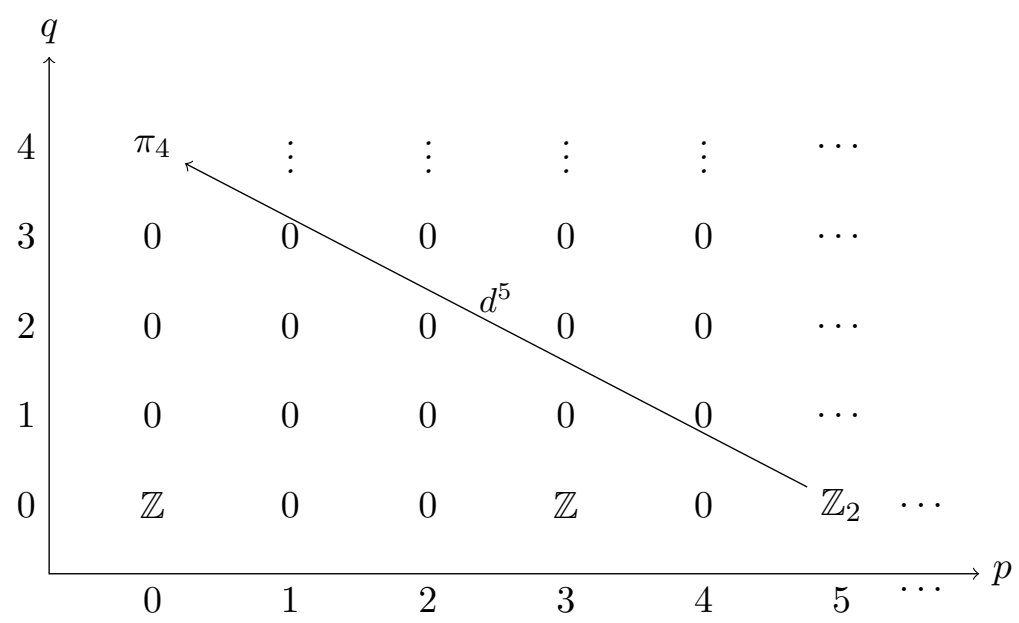

But recall that the space $X_{4}$ is obtained from $S^{3}$ by attaching cells of dimensions greater than 6 , and therefore, since $X_{4}$ has no cells in dimensions 4 and 5 , we already know that $H_{4}\left(X_{4}\right)=$ $H_{5}\left(X_{4}\right)=0$. Hence, the groups $\pi_{4}$ and $\mathbb{Z}_{2}$, on the spectral sequence, must vanish.

It is simple to see that the only nonzero map from/to such groups is $d_{5}: \mathbb{Z}_{2} \rightarrow \pi_{4}$, which must be an isomorphism. Therefore, $\pi_{4}\left(S^{3}\right) \simeq \mathbb{Z}_{2}$.

From the answers given above, other questions arise, regarding Eilenberg-MacLane spaces and also Postnikov Towers.

For instance, there are Whitehead Towers, which are very similar to Postnikov Towers, but in which the spaces $X_{n}$, are such that $\pi_{i}\left(X_{n}\right)=0$, for all $i \leq n$, and the inclusion $X \hookrightarrow X_{n}$ induces isomorphisms in all homotopy groups of dimension greater than $n$. Such tower, which can be thought of as dual to Postnikov's, is also an example of how we can think of Eilenberg-MacLane spaces as base spaces of Homotopy Theory.

Another question is: since CW-complexes determine Postnikov Towers in a unique way, is it possible to make the reverse process? That is, given a Tower satisfying properties 2 and 3 from the Definition 1.3.3, is it always possible to determine a space $X$ which has such diagram as its Postnikov Tower? If so, is such space unique regarding homotopy? The answers to those questions are affirmative and can be seen in the study of the so called Postnikov Systems.

These are some immediate questions that arise, but let us conclude by mentioning one of the most significant applications of Eilenberg-MacLane spaces in algebraic topology: the connection that they make between Homotopy and Homology theories. Indeed, with such spaces we define a new homology sequence of CW-complexes, based simply in the maps between these and EilenbergMacLane spaces. Such homology is equivalent to the singular homology, and thus links these 
two theories. We will explore such topics in Chapter 3, using some of the ideas of such relation in the construction of new Homology and Cohomology Theories, including K-Theory. 


\section{Chapter 2}

\section{Bott's Approach to the Periodicity Theorem}

The Periodicity Theorem for the Classical Groups was first proven by Bott in 1959 [Bot59], using very different tools than the ones which were used to compute the homotopy groups before and were briefly discussed in Chapter 1. Instead, Bott uses the structure of Riemannian symmetric spaces of the classical groups and relies strongly on Morse Theory to obtain information about them. Or, more precisely, on extensions of such a theory.

Morse Theory is a classical branch of Differential Topology which uses well-behaved real valued functions on a finite-dimensional Riemannian manifold to produce a cellular decomposition to such a space. In Section 2.1, we develop some of the main results of the classical Morse Theory, focusing on the ones which will be used in the generalisations developed afterwards. The main references used for this section were [Mil63] and BH13].

In Section 2.2, we present Bott's extension of such a theory, which is now called Morse-Bott Theory. This tool, which was a main instrument in the original proof of the Periodicity Theorems, gives us analogous results to the ones presented in Section 2.1, but asks for less hypothesis on the real-valued function. Afterwards, it was seen that Bott's Periodicity Theorems can be obtained using solely Classical Morse Theory, without this extension. However, we saw this as an opportunity to explore the tool involved in the original proof. The main references for this section were [Bot59], Bot54] and [BH13.

In Section 2.3. we discuss an extension of the theories above to the loop space of a manifold, which was first carried out by Morse himself. The original Morse Theory cannot be directly applied to such a space because it is not a finite dimensional manifold. However, there was an important real-valued function on the loop space which was a great candidate for making the bridge between such theories. The main result of this section is that we can indeed use this function to apply the same procedure used on the Classical Morse Theory to produce a well-behaved cellular decomposition to the loop space.

Finally, in Section 2.4, we show how the information obtained from the algebraic and differential structure of the Classical Groups can be combined with the results developed in the previous 
sections to yield Bott's Periodicity Theorems. The main references in these last two sections were [Mil63] and [Bot59].

\section{$2.1 \quad$ Morse Theory}

Morse Theory can be seen as a tool for understanding the topology of a manifold from its local data. The procedure is intuitively described as one obtained by slicing the manifold and from those slices, and most importantly, from the changes between those slices, understanding the global structure of this space.

This theory has many beautiful and meaningful applications besides the Periodicity Theorem, which include basic results such as "all manifolds admit a CW-structure", and also deep results such as the h-cobordism theorem.

In this section, we assume all manifolds $M$ to be finite dimensional, Hausdorff, second countable, and without boundary. We develop the theory on such spaces, which are the simplest to work with Morse's approach, and the theory developed with them is enough to motivate our next sections.

The idea of "slicing" a manifold comes from looking at a reasonable function from the manifold to $\mathbb{R}$ and observing the pre-images of intervals. We start by understanding what such "reasonable" means, with the definition of Morse functions. Let $f$ be a smooth real-valued function on a manifold $M$.

Definition 2.1.1: A point $p \in M$ is called a critical point of $f$ if the induced map $f_{*}$ : $T_{p} M \rightarrow T_{f(p)} \mathbb{R}$ is zero. Looking at a local coordinate system $\left(x_{1}, \ldots, x_{n}\right)$ in a neighbourhood $U$ of $p$, this means

$$
\frac{\partial f}{\partial x_{1}}(p)=\cdots=\frac{\partial f}{\partial x_{n}}(p)=0
$$

A point $y \in \mathbb{R}$ is called a critical value of $f$ if it contains a critical point in its pre-image.

The critical points of a Morse function are the ones which define the sections that are essentially different from the previous ones. However, we do this analysis looking at specific critical points.

Recall that, if $p$ is a critical point of $f$, the Hessian of $f$ at $p, H_{p} f$ is a symmetric bilinear function on the tangent space $T_{p} M$, which, in terms of local coordinates, is given by

$$
\left(H_{p} f\right)_{i j}=\left(\frac{\partial^{2} f}{\partial x_{i} \partial x_{j}}(p)\right)
$$

Definition 2.1.2: A critical point $p \in M$ of $f$ is called non-degenerate if its Hessian is non-degenerate. That is, the matrix $\left(H_{p} f\right)_{i j}$ is non-singular.

The nullity of a bilinear form is the dimension of its null-space; and the index of a bilinear form is defined to be the maximal dimension of a subspace of $T_{p} M$ in which $H$ is negative definite. 
We also define the index of a non-degenerate critical point as the index of its Hessian.

Since $H$ is symmetric, we can think of the nullity as the multiplicity of zero as an eigenvalue, and the index as the number (counted with multiplicity) of strictly negative eigenvalues. It is clear from the definition that a critical point is non-degenerate if and only if its Hessian has nullity equals to zero. Intuitively, such definition allows us to interpret the index of a non-degenerate critical point as the amount of directions from it, in which the function decreases. The next result shows us that the behaviour of $f$ at such a $p$ can indeed be seen in such a way, and, moreover, that from the index of $p$ we can completely describe $f$ on such neighbourhood.

Lemma 2.1.1: (Morse Lemma) Let $p \in M$ be a non-degenerate critical point of a smooth function $f: M \rightarrow \mathbb{R}$ of index $k$. Then there exists a smooth chart $\phi: U \rightarrow \mathbb{R}^{m}$, where $U$ is an open neighbourhood of $p$, with $\phi(p)=0$ such that if $\phi(x)=\left(x_{1}, \ldots, x_{m}\right)$ for $x \in U$, then

$$
\left(f \circ \phi^{-1}\right)\left(x_{1}, \ldots, x_{m}\right)=f(p)-\left(x_{1}\right)^{2}-\cdots-\left(x_{\lambda}\right)^{2}+\left(x_{\lambda+1}\right)^{2}+\cdots+\left(x_{m}\right)^{2}
$$

A coordinate system $\left(x_{1}, \ldots, x_{n}\right)$ satisfying the relation above is called a Morse coordinate system.

This result allows us to understand the form of the manifold around a non-degenerate critical point, and indeed to interpret the index of such a point as the number of directions from which the function $f$ is decreasing.

Example 2.1.1: Any non-degenerate maximum point of a function on a n-dimensional manifold has index $n$, since the eigenvalues of the Hessian at such a point are strictly negative. Analogously, any non-degenerate minimum point has index zero.

Example 2.1.2: An inflection point on a function $f: \mathbb{R} \rightarrow \mathbb{R}$ is a degenerate critical point. We can see that, in both cases depicted below, the origin is a degenerate critical point.
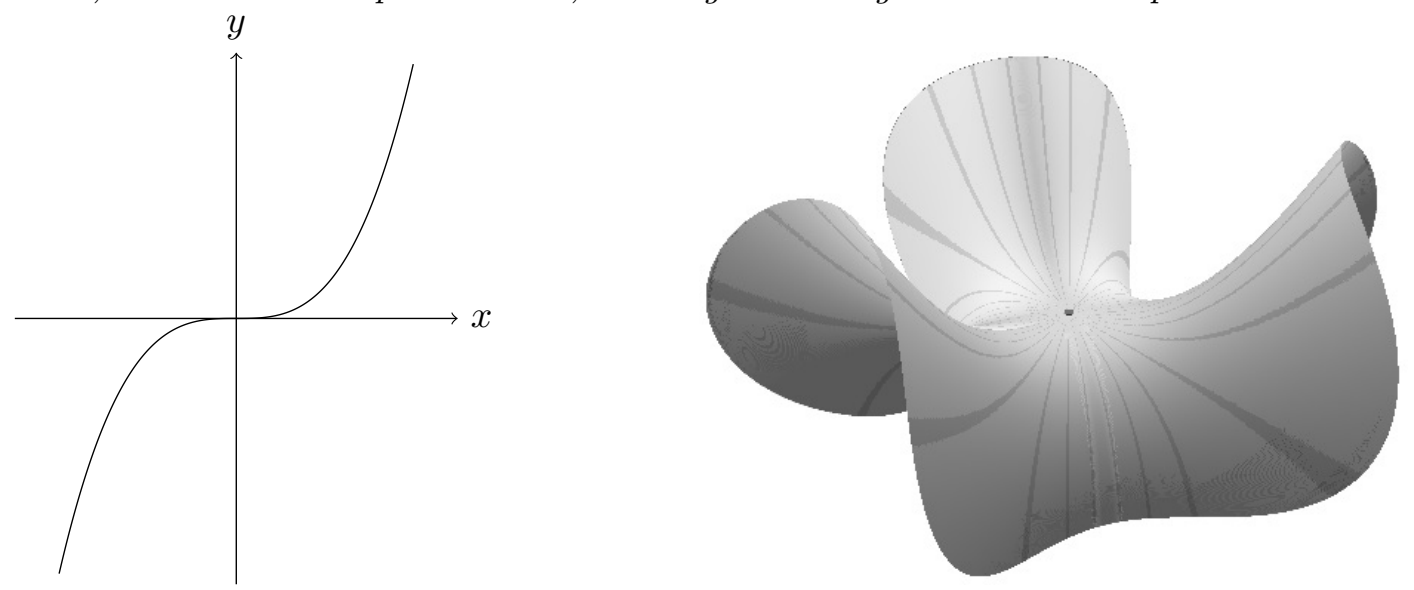

Figure 2.1: Functions $y=x^{3}$ and $z=x^{3}-3 x y^{2}$

To prove Morse Lemma, we will need the following result:

Lemma 2.1.2: $\quad$ Let $f$ be a $C^{\infty}$ function in a convex neighbourhood $V$ of 0 in $\mathbb{R}^{n}$, with $f(0)=0$. 
Then

$$
f\left(x_{1}, \ldots, x_{n}\right)=\sum_{i=1}^{n} x_{i} g_{i}\left(x_{1}, \ldots, x_{n}\right)
$$

for some suitable $C^{\infty}$ functions $g_{i}$ defined in $V$, with $g_{i}(0)=\frac{\partial f}{\partial x_{i}}(0)$.

Proof of Lemma 2.1.2: The result follows from the equalities:

$$
f\left(x_{1}, \ldots, x_{n}\right)=\int_{0}^{1} \frac{d f\left(t x_{1}, \ldots, t x_{n}\right)}{d t} d t=\int_{0}^{1} \sum_{i=1}^{n} \frac{\partial f}{\partial x_{i}}\left(t x_{1}, \ldots, t x n\right) \cdot x_{i} d t
$$

Therefore, we let $g_{i}\left(x_{1}, \ldots, x_{n}\right)=\int_{0}^{1} \frac{\partial f}{\partial x_{i}}\left(t x_{1}, \ldots, t x n\right) d t$

Remark 2.1.2.1: $\quad$ If $g_{i}(0)=0$, for all $i$ in the preceding proposition, then again we can apply the result above to $g_{i}$, and we have

$$
g_{i}\left(x_{1}, \ldots, x_{m}\right)=\sum_{j=1}^{m} x_{j} h_{i j}\left(x_{1}, \ldots, x_{m}\right)
$$

with $h_{i j}(0)=\frac{\partial g_{i}}{\partial x_{j}}(0)=\frac{\partial^{2} f}{\partial x_{i} \partial x_{j}}(0)$. Then $f$ can be written as a quadratic form:

$$
\begin{aligned}
f\left(x_{1}, \ldots, x_{m}\right) & =\sum_{i=1}^{m} x_{i}\left(\sum_{j_{1}}^{m} x_{j} h_{i j}\left(x_{1}, \ldots, x_{m}\right)\right) \\
& =\sum_{i, j} x_{i} x_{j} h_{i j}\left(x_{1}, \ldots, x_{m}\right) \\
& =x^{t} S_{x} x
\end{aligned}
$$

where $x=\left(\begin{array}{c}x_{1} \\ \vdots \\ x_{m}\end{array}\right)$ and $S_{x}=\left(s_{i j}(x)\right)$ is a symmetric matrix with entries

$$
s_{i j}(x)=\frac{1}{2}\left(h_{i j}(x)+h_{j i}(x)\right)
$$

Moving to the proof of Morse Lemma:

Proof of Lemma 2.1.1: The following proof is due to Palais [Pal69] and uses a very famous technique known as Moser's path method.

First, some simplifying assumptions: by replacing $f$ by $f-f(p)$ and choosing a suitable coordinate chart on $M$, we may assume, without loss of generality, that $f$ is defined on a convex neighbourhood $U_{0}$ of $0 \in \mathbb{R}^{m}, f(0)=0$ and $d f(0)=0$. 
Also, we can inductively obtain coordinates such that $A=\left(\frac{\partial^{2} f}{\partial x_{i} \partial x_{j}}(0)\right)$, the hessian at $0 \in \mathbb{R}^{m}$, is a diagonal matrix with the first $k$ entries equal to -1 and the rest equal to 1 . The process of this construction is similar to the usual proof of diagonalization proof for quadratic forms and it is detailed in Mil63 (Lemma 2.2). It is also simple to see from the definition that, if such hessian has only 1 and -1 entries, then the number of negative eigenvalues is precisely the index of the critical point. So we may assume that the hessian of $f$ is of the form described above.

This matrix $A$ defines a quadratic form $\tilde{A}: \mathbb{R}^{m} \rightarrow \mathbb{R}$ which is precisely

$$
\begin{aligned}
\tilde{A}(x) & =x^{t} A x \\
& =-x_{1}^{2}-\cdots-x_{k}^{2}+x_{k+1}^{2}+\cdots+x_{m}^{2}
\end{aligned}
$$

What we will prove is that there are neighbourhoods $U$ and $U^{\prime}$ of 0 contained in $U_{0}$, and a diffeomorphism $\varphi: U \rightarrow U^{\prime}$, such that

$$
f \circ \varphi=\tilde{A}
$$

The path method begins by connecting $f$ and $\tilde{A}$ by a continuous family of functions $F: I \times U_{0} \rightarrow$ $\mathbb{R}$, where we denote $F(t, x)=f_{t}(x)$ with $f_{0}=\tilde{A}$ and $f_{1}=f$. This can be constructed by using paths between the images of $f$ and $\tilde{A}$. For instance, we set

$$
f_{t}=\tilde{A}+t(f-\tilde{A})
$$

The idea of the path method is then to look for a continuous family of diffeomorphisms $\phi$ : $I \times U^{\prime} \rightarrow U$, denoting $\phi(t, x)=\varphi_{t}(x)$, such that

$$
f_{t} \circ \varphi_{t}=f_{0}=\tilde{A}
$$

Then $\varphi=\varphi_{1}$ will be a map satisfying $f \circ \varphi=\tilde{A}$, as we wish.

Rewriting the conditions above in terms of $F$ and $\phi$, we get:

$$
\begin{array}{r}
F(t, x)=\tilde{A}(x)+t(f(x)-\tilde{A}(x)) \\
F(t, \phi(t, x))=\tilde{A}(x)
\end{array}
$$

What we want now is to construct such $\phi$. We can see each $\varphi_{t}$ as the solution of the differential equations

$$
\frac{\partial \phi}{\partial t}(x)=\xi_{t}(\phi(t, x)) \quad \phi(0, x)=x
$$

where the smooth family $\xi_{t}$ is the tangent along the curves $t \rightarrow \varphi_{t}(x)=\phi(t, x)$.

We determine such family $\xi_{t}$ using the relations 2.4 and 2.5, and show that the equation defined above admits a solution. To do that we take the partial derivative with respect to $t$ on both 
sides of 2.5. We can see that, for a $t_{0} \in I$, the derivative in $t_{0}$ is

$$
\begin{aligned}
\left.\frac{d}{d t}\right|_{t=t_{0}} F\left(t, \phi\left(t_{0}, x\right)\right)+\left.\frac{d}{d t}\right|_{t=t_{0}} F\left(t_{0}, \phi(t, x)\right) & =0 \\
\frac{\partial F}{\partial t}\left(t_{0}, \phi\left(t_{0}, x\right)\right)+d f_{t}\left(\varphi_{t}(x)\right)\left(\xi_{t}\left(\varphi_{t}(x)\right)\right) & =0
\end{aligned}
$$

for all $x \in U$. Using 2.4. we see that $\frac{\partial F}{\partial t}(t, x)=f(x)-\tilde{A}(x)$. Simplifying notations and replacing $\varphi(x)$ by $y$ on the relation above, we have

$$
\left(f-\tilde{A}+d f_{t}\left(\xi_{t}\right)\right)(y)=0
$$

Then, setting $g=\tilde{A}-f$, we get

$$
d f_{t}\left(\xi_{t}\right)=g
$$

We show that the equation above admits a solution, by writing both sides of it in a better form.

First, notice that $g(0)=\tilde{A}(0)-f(0)=0$ and $d g(0)=d \tilde{A}(0)-d f(0)=0$. Then $g$ satisfies the hypothesis of Lemma 2.1.2 and even more, of Remark 2.1.2.1. Applying such constructions, we get

$$
g(x)=x^{t} G_{x} x
$$

where $G_{x}$ is a symmetric matrix depending smoothly on $x$.

We now proceed by a process analogous to the one applied in Proposition 2.1 .2 to write $d f_{t}(x)\left(\xi_{t}\right)$ in a better way. By 2.6, we see that $d f_{t}(0)\left(\xi_{t}(0)\right)=g(0)=0$ and therefore

$$
\begin{aligned}
d f_{t}(x)\left(\xi_{t}\right) & =d f_{t}(x)\left(\xi_{t}\right)-d f_{t}(0)\left(\xi_{t}\right) \\
& =\int_{0}^{1} \frac{d}{d s} d f_{t}(s x)\left(\xi_{t}\right) d s \\
& =\int_{0}^{1} \frac{d}{d s}\left(\sum_{j} \xi_{t}^{j} \frac{\partial f_{t}}{\partial x_{j}}(s x)\right) d s \\
& =\int_{0}^{1} \sum_{i j}\left[\xi_{t}^{j} \frac{\partial}{\partial x_{i}}\left(\frac{\partial f_{t}}{\partial x_{j}}(s x)\right) \cdot \frac{d}{d s} s x_{i}\right] d s \\
& =\int_{0}^{1}\left(\sum_{i j} x_{i} \xi_{t}^{j} \frac{\partial^{2} f_{t}}{\partial x_{i} \partial x_{j}}(s x)\right) d s \\
& =\sum_{i j} x_{i} \xi_{t}^{j} \int_{0}^{1} \frac{\partial^{2} f_{t}}{\partial x_{i} \partial x_{j}}(s x) d s \\
& =x^{t} B_{x}^{t} \xi_{t}
\end{aligned}
$$


where $B_{x}^{t}$ is the $m \times m$ matrix with entries

$$
\left(B_{x}^{t}\right)_{i j}=\int_{0}^{1} \frac{\partial^{2} f_{t}}{\partial x_{i} \partial x_{j}}(s x) d s
$$

Notice that, by equation 2.2

$$
B_{0}^{t}=\left(\frac{\partial^{2} f_{t}}{\partial x_{i} \partial x_{j}}(0)\right)
$$

is a diagonal matrix whose $(j, j)$-th entry is

$$
\begin{aligned}
-2+t\left(\left.\frac{\partial^{2} f}{\partial x_{j}^{2}}\right|_{0}+2\right) & =-(2-t) & \text { if } j=1 \ldots, k \\
2+t\left(\left.\frac{\partial^{2} f}{\partial x_{j}^{2}}\right|_{0}-2\right) & =2-t & \text { if } j=k, \ldots, m
\end{aligned}
$$

Therefore, $B_{0}^{t}$ is non-degenerate for all $t \in I$, and there exists a neighbourhood $\tilde{U}$ of $0 \in \mathbb{R}^{m}$, such that $B_{x}^{t}$ is also non-degenerate for all $t$. Then for $x \in \tilde{U}$, we have a unique solution $\xi_{t}$ of

$$
x^{t} B_{x}^{t} \xi_{t}=x^{t} G_{x} x
$$

and this solution depends smoothly on $x$ and $t$. Hence, we have produced a smooth solution to 2.6 defined on $\tilde{U}$. Since $B_{0}^{t}$ is non-degenerate, we know that $\xi_{t}(0)=0$. Hence, by shrinking $\tilde{U}$ we can integrate $\xi_{t}$ and get a smooth family of diffeomorphisms $\varphi_{t}$ from a smaller neighbourhood $U$ of 0 to another neighbourhood $U^{\prime}$ of 0 which satisfies $f_{t} \circ \varphi_{t}=f_{0}=\tilde{A}$ as required.

Using the Morse Lemma, we immediately obtain the following result:

Corollary 2.1.3: $\quad$ Non-degenerate critical points are isolated critical points.

It is simple to verify that such result indeed works, since every critical point has a neighbourhood with Morse coordinates in which the function has no other critical points.

Example 2.1.3: $\quad$ We consider two distinct height functions on the Torus, $\mathbb{T}^{2}$ :

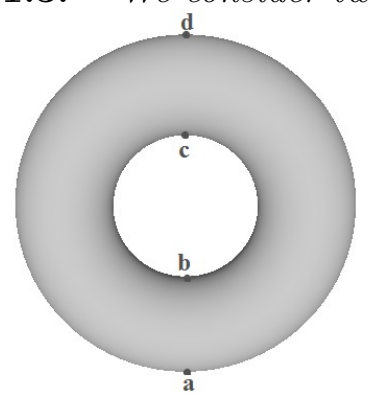

Figure 2.2: Torus layed vertically

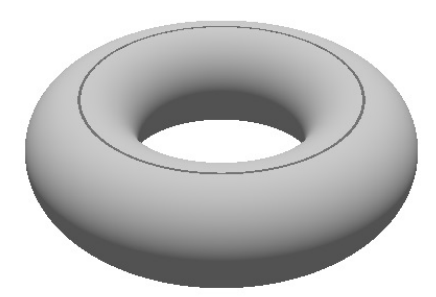

Figure 2.3: Torus layed horizontally

If we consider the height function on the torus positioned as in Figure 2.2 we obtain a Morse function with four non-degenerate critical points: the minimum $a$, two saddle points $b$ and $c$, and the maximum $d$. 
On the other hand, if we think of the height function of the torus lying "horizontally", as in Figure 2.3, then we see that the critical points are the ones on the top and bottom circles, which are maximum and minimum points. Since they are not isolated, we know that the critical points in such a function are degenerate.

Example 2.1.4: We consider two real-valued functions on the sphere $S^{n}$ : the height function $h$, which assigns to each $\left(x_{0}, \ldots, x_{n}\right)$ the number $x_{n}$, and $f$ the map which assigns each such $(n+1)$-tuples to $x_{n}^{2}$.

The height function has two critical points, the north and south poles, which are non-degenerate with indices $n$ and 0 , respectively. On the other hand, the set of critical points of $f$ contains the hole equator of the $n$-sphere and therefore which are clearly non-degenerate critical points.

We now make precise the idea of "slices" we mentioned before:

Definition 2.1.3: If $f$ is a real valued function on $M$, we define the half-plane of $f$ at $a \in \mathbb{R}$ as

$$
M^{a}=f^{-1}(-\infty, a]=\{p \in M: f(p) \leq a\}
$$

Theorem 2.1.4: $\quad$ Let $f$ be a smooth real valued function on $M$ and $a<b$. If the set $f^{-1}[a, b]$ is compact and contains no critical points of $f$, then $M^{a}$ is diffeomorphic to $M^{b}$, and $M^{a}$ is a deformation retract of $M^{b}$. Moreover, there is a smooth diffeomorphism $F: f^{-1}(a) \times[a, b] \rightarrow$ $f^{-1}([a, b])$ such that the diagram

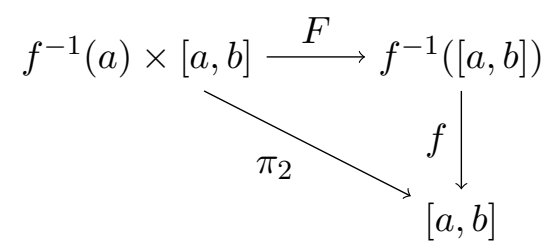

commutes. In particular, all the hal-planes between $M^{a}$ and $M^{b}$ are diffeomorphic.
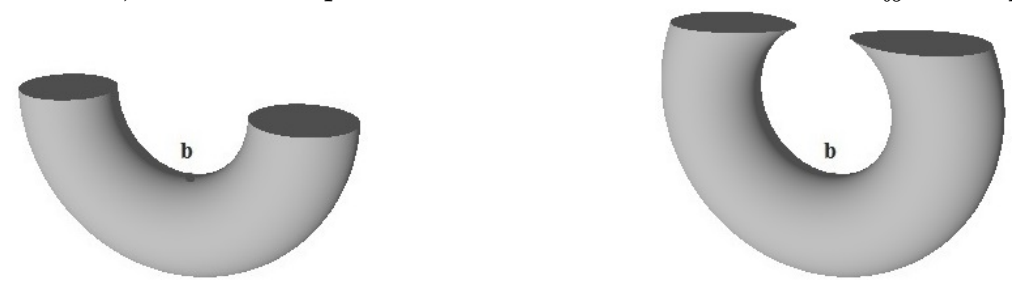

Figure 2.4: $M^{x}, b \leq x<c$

The idea of the proof is to push $M^{b}$ to $M^{a}$ via the trajectories orthogonal to the hypersurfaces $f=$ constant. More precisely, what we do is to deform the manifold along the integral curves of minus the gradient of $f$. Recall that:

Definition 2.1.4: $\quad$ Let $M$ be a Riemmanian manifold with inner product $\langle\cdot, \cdot\rangle$. The gradient of a real valued function $f$ is the vector field, $\nabla f$, on $M$ characterized by the identity

$$
\langle X, \nabla f\rangle=X(f)
$$


for any vector field $X$.

We prove the Morse Lemma using the gradient of the function and the following well-known result:

Lemma 2.1.5: $\quad$ A smooth vector field on $M$ which vanishes outside of a compact set $K \subset M$ generates a unique 1-parameter group of diffeomorphisms of $M$.

The proof of this result is seen in Chapter 1 of [Mil63]. We now move to the proof of the Morse Lemma:

Proof of Theorem 2.1.4: We can see that $\nabla f$ vanishes precisely at the critical points of $f$. What we do here is construct a one-parameter group of diffeomorphisms $\varphi_{t}: M \rightarrow M$ which are closely related to $\nabla f$.

Let $\rho: M \rightarrow \mathbb{R}$ be a smooth function which is equal to $\frac{1}{\|\nabla f\|^{2}}$ throughout the compact set $f^{-1}[a, b]$ and which vanishes outside a compact neighbourhood of this set. Then the vector field

$$
X_{q}=\rho(q) \nabla f_{q}
$$

satisfies the conditions of Lemma 2.1.5. Hence, $X$ generates a 1-parameter group of diffeomorphisms $\varphi_{t}: M \rightarrow M$.

For a fixed $q \in M$, consider the function $t \rightarrow f\left(\varphi_{t}(q)\right)$. If $\varphi_{t}(q)$ lies in the set $f^{-1}[a, b]$, then

$$
\frac{d f\left(\varphi_{t}(q)\right)}{d t}=\left\langle\frac{d \varphi_{t}(q)}{d t}, \nabla f\right\rangle=<X, \nabla f>=1
$$

Thus the correspondence $t \rightarrow f\left(\varphi_{t}(q)\right)$ is linear with derivative 1 , as long as $f\left(\varphi_{t}(q)\right)$ lies between $a$ and $b$. Also, we get

$$
f\left(\varphi_{t}\right)-f\left(\varphi_{0}\right)=\int_{0}^{t} \frac{d}{d s} f\left(\varphi_{s t}\right) d s=t
$$

which implies that $f\left(\varphi_{t}(q)\right)=f(q)+t$.

Then, $\varphi_{b-a}: M \rightarrow M$ is such that, if $f(q)=a, f\left(\varphi_{b-a}(q)\right)=f(q)+b-a=b$, and if $f(q)<a$, then $f\left(\varphi_{b-a}(q)\right)<b$. Therefore, $\varphi_{b-a}$ maps $M^{a}$ into $M^{b}$ and its inverse $\varphi_{a-b}$ maps $M^{b}$ into $M^{a}$. This proves that $M^{a}$ and $M^{b}$ are diffeomorphic.

Also, we use $\varphi_{t}$ to define a proper deformation retraction by considering $r: M^{b} \rightarrow M^{b}$ such that

$$
r(p)=\left\{\begin{array}{cc}
p, & \text { if } p \in M^{a} \\
\varphi_{a-f(q)}(q), & \text { if } p \in M^{b} \backslash M^{a}
\end{array}\right.
$$

This $r$ is a retraction from $M^{b}$ to $M^{a}$ and we see that it is homotopic to the identity via the following diffeomorphism $H: I \times M^{b} \rightarrow M^{b}$

$$
H(t, p)=\left\{\begin{array}{cc}
p, & \text { if } p \in M^{a} \\
\varphi_{t(a-f(q))}(q), & \text { if } p \in M^{b} \backslash M^{a}
\end{array}\right.
$$


Hence, $M^{a}$ is a deformation retract of $M^{b}$.

Finally, we finish the proof of the theorem by defining $F: f^{-1}(a) \times[a, b] \rightarrow f^{-1}([a, b])$ by $F(q, t)=\varphi_{t-a}(q)$. We see from the comments above that, for all $q \in f^{-1}([a, b])$, the point $\varphi_{a-f(q)}(q) \in f^{-1}(a)$. Then $F\left(\varphi_{a-f(q)}(q), f(q)\right)=q$, which shows that $F$ is surjective. From the definition of $\varphi_{t}$, we know that $F\left(x_{1}, t_{1}\right)=F\left(x_{2}, t_{2}\right)$ implies $x_{1}=x_{2}$. Also, if $t_{1} \neq t_{2}$, then

$$
f\left(F\left(x, t_{1}\right)\right)-f\left(F\left(x, t_{2}\right)\right)=f\left(\varphi_{t_{1}}(q)\right)-f\left(\varphi_{t-2}(q)\right)=\left(f(q)+t_{1}\right)-\left(f(q)+t_{2}\right)=t_{1}-t_{2} \neq 0
$$

Hence, $F$ is also injective. Finally, we see that $F$ is an immersion because the gradient lines are transverse to level sets. Therefore, $F$ is a diffeomorphism.

Remark 2.1.5.1: $\quad$ The result above is also valid when $M$ is a manifold with boundary and the proof is basically the same. The only care one has to take is when $f^{-1}([a, b])$ is not contained in the interior of $M$. In this case, to define the vector field which generates the group of diffeomorphisms, one adds an external collar (see [Hir12], section 4.6) to $M$ and finds an open neighbourhood of $f^{-1}([a, b])$ in $M$ union the external collar, such that $\bar{U}$ is compact. Using this, we continue analogously as above.

From the remark above, we get:

Corollary 2.1.6: $\quad$ Let $M$ be a compact smooth manifold with boundary $\partial M=A \amalg B$. If there is a smooth function $f: M \rightarrow[0,1]$ with no critical points such that $f(A)=0$ and $f(B)=1$, then $M$ is diffeomorphic to $A \times[0,1] \approx B \times[0,1]$.

One interesting application of the previous results is:

Proposition 2.1.7: If $M$ is a compact manifold without boundary and $f$ is a smooth function on $M$ with only two critical points, both of which are non-degenerate, then $M$ is homeomorphic to a sphere.

Proof of Proposition 2.1.7: Since $M$ is compact, we know that the two critical points must be the minimum and maximum points. Say that $f\left(p_{-}\right)=z_{-}$is the minimum and $f\left(p_{+}\right)=z_{+}$is the maximum. From Morse Lemma, we know there is a neighbourhood $U_{+}$of $p_{+}$and coordinates $\left(u_{1}, \ldots, u_{m}\right)$, such that $f(x)=z_{+}-\left(u_{1}^{2}+\cdots+u_{m}^{2}\right)$. Hence, for some $b<z_{+}$, the set $D_{+}=$ $f^{-1}\left(\left[b, z_{+}\right]\right)$is diffeomorphic to the closed $m$-disk $D^{m}$. Analogously, there is $a>z_{-}$, such that $D_{-}=f^{-1}\left(\left[z_{-}, a\right]\right)$ is also diffeomorphic to $D^{m}$. By the remark above, $f^{-1}([a, b])$ is diffeomorphic to $S^{m-1} \times[0,1]$.

We then construct a homeomorphism to $S^{n}$, also by decomposing it in three parts: disjoint neighbours $B_{+}$and $B_{-}$of the north and south poles, respectively, and $C=S^{n}-\operatorname{int}\left(B_{+} \cup B_{-}\right) \approx$ $S^{m-1} \times[0,1]$. The Morse Lemma gives us a diffeomorphism $h_{+}: D_{+} \rightarrow B_{+} \approx D^{m}$ and we can extend $\left.h_{+}\right|_{\partial D_{+}}$to a diffeomorphism from $\partial D_{+} \times[0,1] \rightarrow \partial B_{+} \times[0,1]$. Since $f^{-1}([a, b])$ is diffeomorphic to $\partial D_{+} \times[0,1]$, this gives an extension of $h_{+}$to a homeomorphism

$$
f: D_{+} \cup f^{-1}([a, b]) \rightarrow B_{+} \cup C
$$


Finally, if we let $g_{0}=\left.h\right|_{\partial D_{-}}$we can extend $g_{0}$ radially to a homeomorphism $g: D_{-} \approx D^{m} \rightarrow$ $B_{-} \approx D^{m}$, by letting

$$
g(x)= \begin{cases}0 & \text { if } x=0 \\ \|x\| g_{0}\left(\frac{x}{\|x\|}\right) & \text { if } x \neq 0\end{cases}
$$

Putting together $h$ and $g$ we get a homeomorphism from $M$ to $S^{m}$.

It is also true, although the proof is more complicated, that the result above holds even when the critical points are degenerate (see [MW64]). It is not true, however, that a manifold $M$ with such properties is diffeomorphic to $S^{m}$. For instance, Milnor (see [Mil56]) produced spaces which are homeomorphic to $S^{7}$ but do not have its usual differentiable structure.

Theorem 2.1.4 gives a topological description of how a manifold "evolves" between two "heights" when there are no critical points between them. However, Morse Theory gives us even more: how the manifold changes when passing through a non-degenerate critical point:

Theorem 2.1.8: $\quad$ Let $f: M \rightarrow \mathbb{R}$ be a smooth function, and let $p$ be a non-degenerate critical point of $f$ with index $k$. Setting $f(p)=c$, suppose that $f^{-1}[c-\epsilon, c+\epsilon]$ is compact and contain no critical point of $f$ other than $p$, for some $\epsilon>0$. Then, for all sufficiently small $\epsilon>0$, the set $M^{c+\epsilon}$ has the homotopy type of $M^{c-\epsilon}$ with a $k$-cell attached.
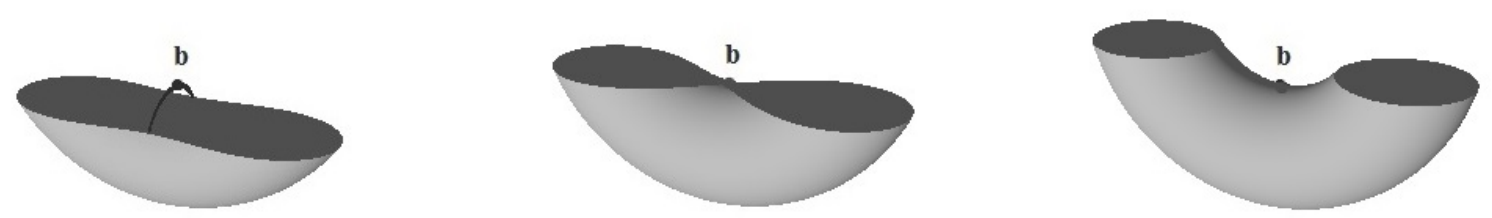

The idea of this proof is to introduce a new function $F: M \rightarrow \mathbb{R}$ which coincides with $f$, except that $F<f$ in a small neighbourhood of the critical point $p$. Thus, the region $F^{-1}(-\infty, c-\varepsilon]$ will consist of $M^{c-\varepsilon}$ together with a region $H$ near $p$.

Figure 2.5 is a diagram which expresses the intuitive idea of the function $F$ on the torus. In this figure, $H$ is represented by the horizontally shaded region.

Choosing a suitable cell $e^{\lambda} \subset H$, a direct argument (i.e., pushing along the horizontal lines) shows that $M^{c-\varepsilon} \cup e^{\lambda}$ is a deformation retract of $M^{c-\varepsilon} \cup H$. Applying Theorem 2.1.4, we see that $M^{c-\varepsilon} \cup H$ is a deformation retract of $M^{c+\varepsilon}$.

Proof of Theorem 2.1.8: Let $\phi: U \rightarrow \mathbb{R}^{m}$ be the smooth chart given by the Morse Lemma with $\phi(x)=\left(x_{1}, \ldots, x_{m}\right)$. To facilitate notation, we set

$$
\begin{aligned}
& \xi^{2}=x_{1}^{2}+\cdots+x_{k}^{2} \\
& \eta^{2}=x_{k+1}^{2}+\cdots+x_{m}^{2}
\end{aligned}
$$

From Morse Lemma, we see that $f \circ \phi^{-1}\left(x_{1}, \ldots, x_{m}\right)=c-\xi^{2}+\eta^{2}$, which we abbreviate by $f=c-\xi^{2}+\eta^{2}$.

We choose $0<\varepsilon<1$ small enough to guarantee that $f^{-1}([c-\varepsilon, c+\varepsilon])$ is compact and contains 


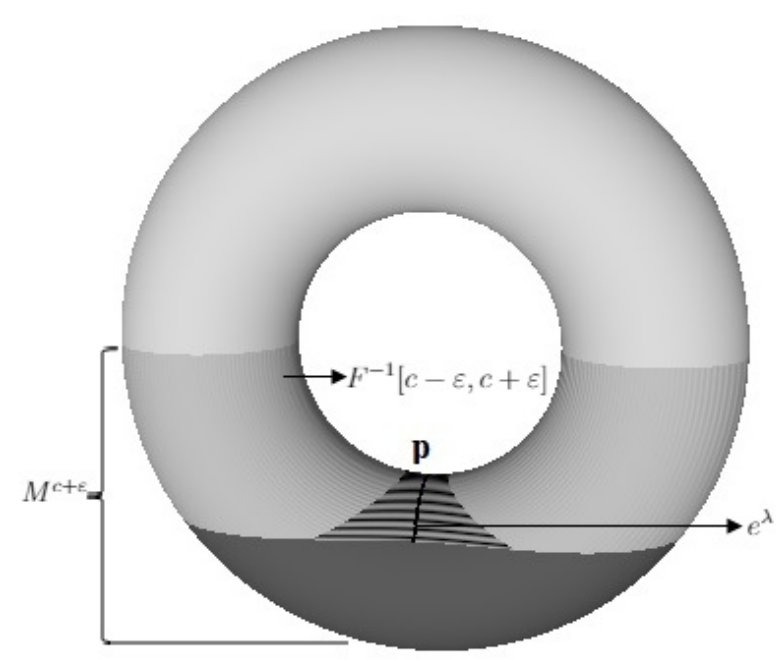

Figure 2.5: Diagram of $F$ on the torus

no critical points other than $p$, but also making sure that $\phi(U)$ contains the disk of radius $2 \varepsilon$.

With this terminology,

$$
\begin{aligned}
& M^{c-\varepsilon} \cap U=\left\{x \in U: c-\varepsilon \geq f(x)=c-\xi^{2}+\eta^{2}\right\}=\left\{x \in U: \xi^{2}-\eta^{2} \geq \varepsilon\right\} \\
& M^{c+\varepsilon} \cap U=\left\{x \in U: c+\varepsilon \geq f(x)=c-\xi^{2}+\eta^{2}\right\}=\left\{x \in U: \xi^{2}-\eta^{2} \leq \varepsilon\right\}
\end{aligned}
$$

The cell to which we refer on the theorem is

$$
e^{k}=\left\{x \in U: \xi^{2} \leq \varepsilon \text { and } \eta^{2}=0\right\}
$$

and we see that it is attached to $M^{c-\varepsilon}$ by the remarks above.

To show that $M^{c+\varepsilon}$ is homotopy equivalent to $M^{c-\varepsilon} \cup e^{k}$, we construct another function $F$, whose half-planes $\left.\left.F^{-1}(]-\infty, c-\varepsilon\right]\right)$ and $\left.\left.F^{-1}(]-\infty, c+\varepsilon\right]\right)$ are easier to understand. Intuitively, we create $F$ by decreasing the value of a specific neighbourhood of $p$ which is contained in $U$. To do that, we consider a function $\mu: \mathbb{R} \rightarrow \mathbb{R}$ such that $\mu(0) \geq \varepsilon, \mu(r)=0$, if $r \geq 2 \varepsilon$ and $-1 \leq$

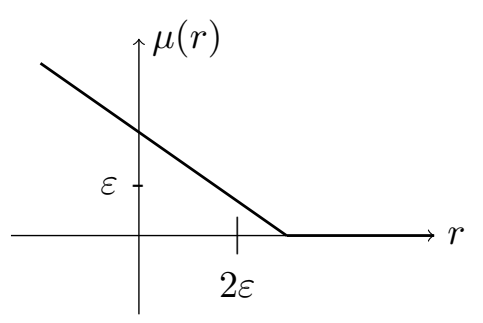
$\mu^{\prime}(r) \leq 0$. Defining

$$
F= \begin{cases}f & \text { outside of } U \\ f-\mu\left(\xi^{2}+2 \eta^{2}\right) & \text { inside of } U\end{cases}
$$

we obtain the desired function. Notice that $F(p)=f(p)-\mu(0)<c-\varepsilon$ and $F(x) \leq f(x)$, for all $x$. Moreover, $F$ has the following properties:

1. $\left.\left.F^{-1}(]-\infty, c+\varepsilon\right]\right)$ is precisely $M^{c+\varepsilon}$ : since $F \leq f$, we know that $\left.\left.M^{c+\varepsilon} \subset F^{-1}(]-\infty, c+\varepsilon\right]\right)$. Let's look at a point $x$ with $F(x) \leq c+\varepsilon$. If $x$ is on the set of points where $F=f$, then $x \in M^{c+\varepsilon}$ as well. If that's not the case, then $x \in U$ and $\xi^{2}-2 \eta^{2}<2 \varepsilon$. But

$$
f(x)=c-\xi^{2}+\eta^{2} \leq c+\frac{1}{2} \xi^{2}+\eta^{2} \leq c-\varepsilon
$$


and therefore $x \in M^{c+\varepsilon}$. From this we conclude the equivalence.

2. $F$ and $f$ have the same critical points: the only set where $F$ and $f$ may not coincide is $U$. For $x \in U, F(x)=f(x)-\mu\left(\xi^{2}-2 \eta^{2}\right)=c-\xi^{2}+\eta^{2}-\mu\left(\xi^{2}-2 \eta^{2}\right)$. From calculus, we see

$$
D F=\frac{\partial F}{\partial \xi^{2}} d \xi^{2}+\frac{\partial F}{\partial \eta^{2}} d \eta^{2}
$$

From the relation $F(x)=c-\xi^{2}+\eta^{2}-\mu\left(\xi^{2}-2 \eta^{2}\right)$, we see

$$
\begin{aligned}
& \frac{\partial F}{\partial \xi^{2}}=-1-\mu^{\prime}\left(\xi^{2}-2 \eta^{2}\right) \cdot 1 \\
& \frac{\partial F}{\partial \eta^{2}}=1-\mu^{\prime}\left(\xi^{2}-2 \eta^{2}\right) \cdot(-2)
\end{aligned}
$$

Since $-1<\mu^{\prime} \leq 0$, we see that $\frac{\partial F}{\partial \xi^{2}}<0$ and $\frac{\partial F}{\partial \eta^{2}} \geq 1$, and therefore $D F$ is only be zero if $d \xi^{2}=d \eta^{2}=0$, which happens if and only if $x$ is a critical point of $f$ in $U$, ie. $x=p$.

3. $F^{-1}([c-\varepsilon, c+\varepsilon]) \subset f^{-1}([c-\varepsilon, c+\varepsilon])$ : since $F \leq f$, we know that $f^{-1}([-\infty, c-\varepsilon]) \subset$ $F^{-1}([-\infty, c-\varepsilon])$. Then

$$
\begin{aligned}
F^{-1}([c-\varepsilon, c+\varepsilon]) & =F^{-1}([-\infty, c+\varepsilon])-F^{-1}([-\infty, c-\varepsilon]) \\
& =M^{c+\varepsilon}-F^{-1}([-\infty, c-\varepsilon]) \\
& \subset M^{c+\varepsilon}-f^{-1}([-\infty, c-\varepsilon]) \\
& =f^{-1}([c-\varepsilon, c+\varepsilon])
\end{aligned}
$$

Using the properties above, we see that $F^{-1}([c-\varepsilon, c+\varepsilon])$ is a deformation retract of $M^{c+\varepsilon}$, since $F^{-1}([c-\varepsilon, c+\varepsilon])$ satisfies the hypothesis of Theorem 2.1.4. We now show that $M^{c-\varepsilon} \cup e^{k}$ is a deformation retract of $F^{-1}([-\infty, c-\varepsilon])$.

Let $H$ denote the closure of $\left.F^{-1}(]-\infty, c-\varepsilon\right)-M^{c-\varepsilon}$, then $H$ can be seen as a handle attached to $M^{c-\varepsilon}$ which contains $e^{k}$. We now construct a deformation retraction $r_{t}: M^{c-\varepsilon} \cup H \rightarrow M^{c-\varepsilon} \cup H$ as the identity outside $U$ and, in $U$, it will be defined as:

Case 1: Within the region $\xi^{2} \leq \varepsilon$ let $r_{t}$ correspond to the transformation

$$
\left(x_{1}, \ldots, x_{m}\right) \rightarrow\left(x_{1}, \ldots, x_{k}, t x_{k+1}, \ldots, t x_{m}\right)
$$

Then $r_{1}$ is the identity and $r_{0}$ maps the entire region into $e^{k}$. The fact that each $r_{t}$ maps $F^{-1}(-\infty, c-\varepsilon]$ into itself follows from the inequality $\frac{\partial F}{\partial \eta^{2}}>0$

Case 2: Within the region $\varepsilon<\xi^{2} \leq \eta^{2}+\varepsilon$ let $r_{t}$ correspond to the transformation

$$
\left(x_{1}, \ldots, x_{m}\right) \rightarrow\left(x_{1}, \ldots, x_{\lambda}, s_{t} x_{\lambda+1}, \ldots, s_{t} x_{m}\right)
$$


where the number $s_{t} \in[0,1]$ is defined by

$$
s_{t}=t+(1-t)\left(\frac{\xi^{2}-\varepsilon}{\eta^{2}}\right)^{\frac{1}{2}}
$$

Thus, $r_{1}$ is again the identity and $r_{0}$ maps the entire region into the hypersurface $f^{-1}(c-\varepsilon)$. We still need to verify that the functions $s_{t} u_{i}$ remain continuous as $\xi^{2} \rightarrow \varepsilon, \eta^{2} \rightarrow 0$. This definition coincides with that of Case 1 when $\xi^{2}=\varepsilon$.

Case 3: Within the region $\eta^{2}+\varepsilon \leq \xi^{2}$ (i.e., within $M^{c-\varepsilon}$ ) let $r_{t}$ be the identity. This coincides with our preceding definition when $\xi=\eta+\varepsilon$.

This completes the proof that $M^{c-\varepsilon} \cup e^{k}$ is a deformation retract of $F^{-1}(-\infty, c-\varepsilon)$, which we proved to be a deformation retract of $M^{c+\varepsilon}$.

The proof above can be easily modified to also show:

Lemma 2.1.9: If there are $j$ critical points $p_{1}, \ldots, p_{j}$ in the level set $f^{-1}(c)$ with indices $k_{1}, \ldots, k_{j}$, respectively, then

$$
M^{c+\varepsilon}=M^{c-\varepsilon} \cup_{f_{1}} D^{k_{1}} \cup_{f_{2}} \cdots \cup_{f_{j}} D^{k_{j}}
$$

for some collection of attaching maps $f_{1}, \ldots, f_{j}$.

This is seen by using the same strategy used to construct $F$ on the proof of Theorem 2.1.8, to define a map with the same critical points of $f$, such that no two of them are in the same level set. Then we combine Theorems 2.1.4 and 2.1.8 to obtain the result.

Lemma 2.1.10: $\quad M^{c}$ is a deformation retract of $M^{c+\varepsilon}$.

Using the function $F$ defined on the proof of Theorem 2.1.8, we know that $\left.\left.F^{-1}(]-\infty, c\right]\right)$ is a deformation retract of $\left.\left.F^{-1}(]-\infty, c+\varepsilon\right]\right)=M^{c+\varepsilon}$, by Theorem 2.1.4 and we adapt the argument above to exhibit a deformation retract of $\left.\left.F^{-1}(]-\infty, c\right]\right)$ to $M^{c}$, which gives us the desired result.

Combining the results above, we get:

Corollary 2.1.11: $\quad$ Let $f: M \rightarrow \mathbb{R}$ be a smooth function and let $a, b \in \mathbb{R}$ be such that $f^{-1}([a, b])$ is compact and has no degenerate critical points. Then $M^{b}$ is homotopy equivalent to $M^{a}$ with a finite number of cells attached:

$$
M^{b}=M^{a} \cup_{f_{1}} D^{k_{1}} \cup_{f_{2}} \cdots \cup_{f_{l}} D^{k_{l}}
$$

where $k_{1}, \ldots, k_{l}$ are the indices of the critical points and $f_{1}, \ldots, f_{l}$, some collection of attaching maps.

Indeed, since $f^{-1}([a, b])$ is compact and non-degenerate critical points are isolated, there are only finitely many such points. Then we can recursively apply Theorem 2.1.8 and the lemmas above to obtain the desired relation.

One could ask if this result can be extended in a way that we do not need to hold to the "finite 
amount of critical points" condition. The result below is one such extension:

Theorem 2.1.12: If $f$ is a differentiable function on a manifold $M$ with no degenerate critical points, and if each $M^{a}$ is compact, then $M$ has the homotopy type of a $C W$-complex, with one cell of index $\lambda$ for each critical point of index $\lambda$.

Figure 2.6 is a scheme representing the CW structure we obtain on the torus using this theorem on the height function.
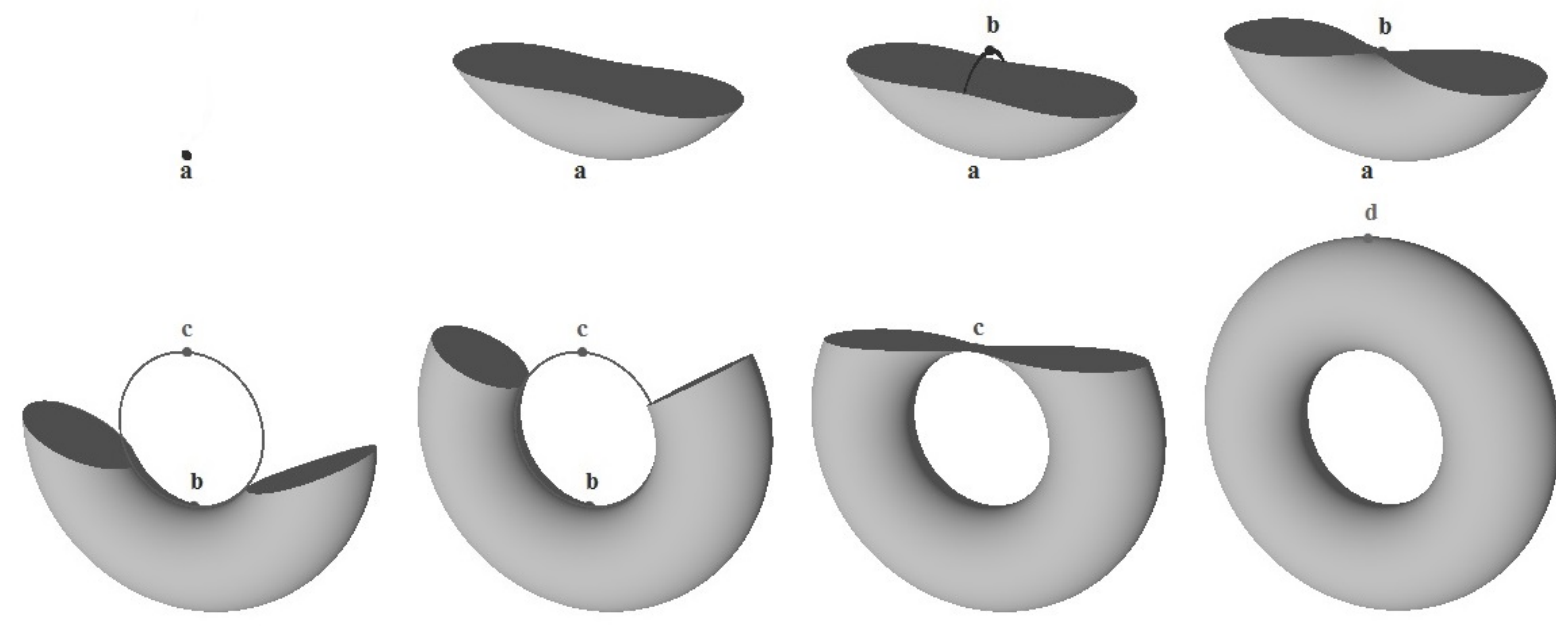

d

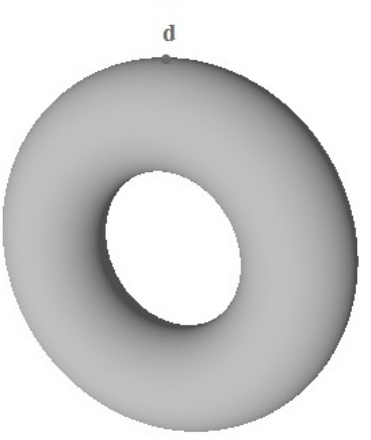

Figure 2.6: CW complex structure on the torus given by the height function

The proof of the Theorem above is based on the following two lemmas regarding a topological space $X$ with a cell attached.

Lemma 2.1.13: Let $\varphi_{0}$ and $\varphi_{1}$ be homotopic maps from the sphere $\partial e^{\lambda}$ to $X$. Then the identity map of $X$ extends to a homotopy equivalence

$$
k: X \cup_{\varphi_{0}} e^{\lambda} \rightarrow X \cup_{\varphi_{1}} e^{\lambda}
$$

Proof of Lemma 2.1.13; We define $k$ by the formulas

$$
\begin{array}{ll}
k(x)=x & \text { for } x \in X \\
k(t u)=2 t u & \text { for } 0 \leq t \leq \frac{1}{2} \text { and } u \in \partial e^{\lambda} \\
k(t u)=\varphi_{2-2 t}(u) & \text { for } \frac{1}{2} \leq t \leq 1 \text { and } u \in \partial e^{\lambda}
\end{array}
$$

where $\varphi_{t}$ denotes the homotopy between $\varphi_{0}$ and $\varphi_{1}$.

A corresponding map $l: X \cup_{\varphi_{1}} e^{\lambda} \rightarrow X \cup_{\varphi_{0}} e^{\lambda}$ is defined by similar formulas and it is not difficult to verify that the compositions $k \circ l$ and $l \circ k$ are homotopic to the respective identity maps. Thus $k$ is a homotopy equivalence. 
Lemma 2.1.14: $\quad$ Let $\varphi: \partial e^{\lambda} \rightarrow X$ be an attaching map. Any homotopy equivalence $f: X \rightarrow Y$ extends to a homotopy equivalence

$$
F: X \cup_{\varphi} e^{\lambda} \rightarrow Y \cup_{f \circ \varphi} e^{\lambda}
$$

Proof of Lemma 2.1.14: Define $F$ by the conditions

$$
\left.F\right|_{X}=\left.f \quad F\right|_{e^{\lambda}}=\text { identity }
$$

Let $g: Y \rightarrow X$ be a homotopy inverse to $f$ and define

$$
G: Y \cup_{f \circ \varphi} e^{\lambda} \rightarrow X \cup_{\varphi} e^{\lambda}
$$

by the analogous conditions: $\left.G\right|_{Y}=g$ and $\left.G\right|_{e^{\lambda}}=$ identity.

Since $g \circ f \circ \varphi$ is homotopic to $\varphi$, it follows from Lemma2.1.13 that there is a homotopy equivalence

$$
k: X \cup_{g f \varphi} e^{\lambda} \rightarrow X \cup_{\varphi} e^{\lambda}
$$

We will first prove that the composition $k G F: X \cup_{g f \varphi} e^{\lambda} \rightarrow X \cup_{\varphi} e^{\lambda}$ is homotopic to the identity map, using the homotopy $h_{t}$ between $g f$ and the identity. From the definitions of $k, G$ and $F$, we can see that

$$
\begin{array}{ll}
k F G(x)=g f(x) & \text { for } x \in X \\
k F G(t u)=2 t u & \text { for } 0 \leq t \leq \frac{1}{2} \text { and } u \in \partial e^{\lambda} \\
k F G(t u)=h_{2-2 t} \varphi(u) & \text { for } \frac{1}{2} \leq t \leq 1 \text { and } u \in \partial e^{\lambda}
\end{array}
$$

We then construct the required homotopy $q_{\tau}: X \cup_{\varphi} e^{\lambda} \rightarrow X \cup_{\varphi} e^{\lambda}$ by the formulas

$$
\begin{array}{ll}
q_{\tau}(x)=h_{\tau}(x) & \text { for } x \in X \\
q_{\tau}(t u)=\frac{2}{1+\tau} t u & \text { for } 0 \leq t \leq \frac{1+\tau}{2} \text { and } u \in \partial e^{\lambda} \\
q_{\tau}(t u)=h_{2-2 t+\tau} \varphi(u) & \text { for } \frac{1+\tau}{2} \leq t \leq 1 \text { and } u \in \partial e^{\lambda}
\end{array}
$$

Therefore $F$ has a left homotopy inverse.

Now we prove that $F$ is a homotopy equivalence using the following claim about homotopy inverses which is purely formal:

Claim: If a map $F$ has a left homotopy inverse $L$ and a right homotopy inverse $R$, then $F$ is a homotopy equivalence and $R$ (or $L$ ) is a homotopy inverse. 
Proof of claim: The relations

$$
L F \simeq \text { identity, } \quad F R \simeq \text { identity }
$$

imply that $L \simeq L(F R)=(L F) R \simeq R$. Consequently, $R F \simeq L F \simeq$ identity, which proves that $R$ is a two-sided inverse.

We now complete the proof as follows: the relation $k F G \simeq$ identity asserts that $F$ has a left homotopy inverse; and a similar proof shows that $G$ has a left homotopy inverse.

1: Since $k$ is a homotopy equivalence, we know it has a left inverse. Also, we have shown that $k(G F) \simeq$ identity. Thus, by the claim above, it follows that $(G F) k \simeq$ identity.

2: Since $G(F k) \simeq$ identity, and we construct a left inverse to $G$ by the same method we used on $F$, it follows that $(F k) G \simeq$ identity.

3: Finally, since $(F k) G \simeq$ identity, and $F$ has $k G$ as left inverse also, it follows that $F$ is a homotopy equivalence.

Using such results, we prove Theorem 2.1 .12 the following way:

Proof of Theorem 2.1.12: Since each $M^{a}$ is compact, we know that $f\left(M^{a}\right)$ is compact, and therefore, assumes a minimum value, $c_{1}$. Then $M^{a}=\emptyset$, for all $a<c_{1}$, and the critical values of $f: M \rightarrow \mathbb{R}$ are organised in a sequence $c_{1}<c_{2}<c_{3}<\ldots$ with no cluster points, since each $M^{a}$ is compact.

We define the desired CW-complex "inductively", looking at the CW-complex which is related to each half-space by Theorems 2.1.4, 2.1.8 and Lemma 2.1.10. The base case is simple since $M^{c_{1}}$ is a disjoint union of a finite set of points, which is clearly a CW-complex. Now suppose that for each $i=1, \ldots, n-1$, we have constructed a homotopy equivalence $\phi_{i}$ between $M^{c_{i}}$ and a CW-complex $K_{i}$ satisfying

$$
\left.K_{i} \subset K_{i+1} \quad \phi_{i+1}\right|_{M^{c_{i}}}=\phi_{i}
$$

We now construct $\phi_{n}$ : by Theorem 2.1.8 and Lemma 2.1.10, we know that $M^{c_{n}}$ has the same homotopy type of $M^{c_{n}-\varepsilon} \cup_{\varphi_{1}} e^{\lambda_{1}} \cup \cdots \cup_{\varphi_{j}(n)} e^{\lambda_{j(n)}}$ for certain maps $\varphi_{1}, \ldots, \varphi_{j(n)}$ when $\varepsilon$ is small enough, and there is a homotopy equivalence $h: M^{c-\varepsilon} \rightarrow M^{c_{n-1}}$, by Theorem 2.1.4 and Lemma 2.1.10.

Then each $\phi_{n-1} \circ h \circ \varphi_{j}$ is homotopic, by a cellular approximation, to a map

$$
\psi_{j}: \partial e^{\lambda_{j}} \rightarrow\left(\lambda_{j}-1\right)-\text { skeleton of } K_{n-1}
$$

Then $K_{n}=K_{n-1} \cup_{\psi_{1}} e^{\lambda_{1}} \cup \cdots \cup_{\psi_{j(n)}} e^{\lambda_{j(n)}}$ is a CW-complex and has the same homotopy type as $M^{c_{n}}$, by Lemmas 2.1 .13 and 2.1 .14 and the homotopy equivalence $\phi_{n}$ induced by the relations above extends $\phi_{n-1}$.

If $M$ is compact, there will be a last critical value. Doing the process above at such maximum of $f$, completes our proof. If $M$ is not compact, but all critical points lie in one of the compact 
sets $M^{a}$, then a proof similar to Theorem 2.1.4 shows that the set $M^{a}$ is a deformation retract of $M$ so the proof is again complete.

If there are infinitely many critical points then the above construction gives us an infinite sequence of homotopy equivalences

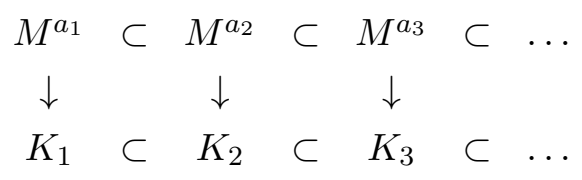

each extending the previous one. Let $K$ denote the union of the $K_{i}$ in the direct limit topology, i.e., the finest possible compatible topology, and let $g: M \rightarrow K$ be the limit map. Then, since all spheres $S^{n}$ are compact, their image under any map is also compact, and therefore, it is contained in some $M^{a_{i}}$ which is homotopy equivalent to $K_{i}$. This implies that $g$ induces isomorphisms of homotopy groups in all dimensions, and therefore, by Whitehead's Theorem, 1.3.6, $g$ is a homotopy equivalence.

The results above are very important and impressive, since they show how to understand the local structure of a manifold looking at non-degenerate critical points of smooth functions. The best case in which we can apply the Theorems above, then generates the following:

Definition 2.1.5: A Morse function on a smooth manifold is a smooth function whose critical points are all non-degenerate.

The tools and results developed above are enough to motivate and understand the generalisations which are necessary for Bott's proof of the Periodicity Theorem. However, I will close this section by answering a question that came to me while studying Morse Theory: now that we have all this apparatus developed for Morse functions, how can we know when such a function exists? The next results show us that, in fact, there are infinitely many Morse functions on any manifold and they are actually generic.

The Whitney embedding theorem states that any smooth manifold $M$, say of dimension $m$, can be embedded in an euclidean space $\mathbb{R}^{r}$, for some $r>m$. We use any such maps to construct Morse functions. Denoting a point $x \in M$ by its coordinates $\left(x_{1}, \ldots, x_{r}\right) \in \mathbb{R}^{r}$ when seen inside $\mathbb{R}^{r}$, we have:

Theorem 2.1.15: For almost all $a=\left(a_{1}, \ldots, a_{r}\right) \in \mathbb{R}^{r}$ (all but a set of measure zero) the function $f: M \rightarrow \mathbb{R}$ given by

$$
f(x)=a_{1} x_{1}+a_{2} x_{2}+\cdots+a_{r} x_{r}
$$

is a Morse function.

We prove the result above from the following:

Lemma 2.1.16: $\quad$ Let $f: U \rightarrow \mathbb{R}$ be a smooth function on an open set $U \subset \mathbb{R}^{m}$. For almost all 
$a=\left(a_{1}, \ldots, a_{m}\right)$, the function

$$
f_{a}(x)=f(x)-\sum_{j=1}^{m} x_{j} a_{j}
$$

is a Morse function.

Proof of Lemma 2.1.16: If $p \in \mathbb{R}^{m}$ is a critical point of $f_{a}$, then

$$
0=\left(d f_{a}\right)_{p}=\left(\frac{\partial f}{\partial x_{1}}(p), \ldots, \frac{\partial f}{\partial x_{m}}(p)\right)-a
$$

which implies that $p$ is a critical point of $f_{a}$, if, and only if, $g(p)=\left(\frac{\partial f}{\partial x_{1}}(p), \ldots, \frac{\partial f}{\partial x_{m}}(p)\right)=a$.

From the definition of $f_{a}$, we can see that $\frac{\partial^{2} f_{a}}{\partial x_{i} \partial x_{j}}=\frac{\partial^{2} f}{\partial x_{i} \partial x_{j}}$, which implies that the Hessian of $f_{a}$ in a point $p$ is precisely $d g_{p}$. Therefore, to guarantee that no critical points are degenerate, it's enough to choose $a$ in the set of regular values of $g$, which, by Sard's Theorem, is $\mathbb{R}^{m}$ minus a set of Lebesgue measure zero.

Proof of Theorem 2.1.15: Let $\left(x_{1}, \ldots, x_{r}\right)$ be the coordinates of a point $x \in M \subset \mathbb{R}^{r}$. Since we are considering an embedding of $M$, we know that the induced map on the tangent spaces $T_{x} M \hookrightarrow T_{x} \mathbb{R}^{r}$ is injective, which implies that its dual $T_{x}^{*} \mathbb{R}^{r} \rightarrow T_{x}^{*} M$ is surjective. This implies that for some neighbourhood $U$ of $x,\left.T^{*} M\right|_{U}$ is spanned by some linearly independent $d x_{j_{1}}, \ldots, d x_{j_{m}}$. Through this, we can see that $\left(x_{j_{1}}, \ldots, x_{j_{m}}\right)$ are also linearly independent and hence form a coordinate system on $U$.

To facilitate notation, let's assume that $\left(x_{1}, \ldots, x_{m}\right)$ forms a coordinate system in $U$. By Lemma 2.1.16, we know that for almost all $\left(a_{1}, \ldots, a_{r}\right) \in \mathbb{R}^{r}$ we get a Morse function

$$
f_{a}(x)=0-\sum_{j=1}^{r} x_{j} a_{j}
$$

which implies that $-f_{a}(x)$ is a Morse function in $U$.

We now find a countable cover $\left\{U_{j}\right\}_{j}^{\infty}$ of $M$ by neighbourhoods of the form above. Each $A_{j}=$ $\left\{a \in \mathbb{R}^{r}: f_{a}\right.$ is not a Morse function on $\left.U_{j}\right\}$ has measure zero and therefore $A=\bigcup_{j} A_{j}$ has also measure zero. Hence, we see that $f_{a}$ is a Morse function on $M$ for all $a \in \mathbb{R}^{r}-A$.

Looking at Lemma 2.1.16, we see that the function $f_{a}$ can be made to be arbitrarily close to $f$ by choosing $a \in \mathbb{R}^{m}$ close to the origin. This result, which implies that all smooth functions on an open set of $\mathbb{R}^{m}$ can be arbitrarily approximated by Morse functions, can be generalised on an arbitrary manifold:

Proposition 2.1.17: Any bounded smooth function $f: M \rightarrow \mathbb{R}$ can be uniformly approximated by a Morse function $g$. Furthermore, $g$ can be chosen so that the $i$-th derivatives of $g$ on the compact set $K$ uniformly approximate the corresponding derivatives of $f$, for $i \leq k$.

This shows that the set of Morse functions is actually dense on the set of smooth real-functions 
with the compact-open topology. To see a proof of the result above, see BH13] Theorem 5.27.

We close this section stating two very important results on manifolds which are immediate if we combine the theory developed above with Whitney's Theorem, which states that an ndimensional manifold $M$ can be embedded differentiably as a closed submanifold of $\mathbb{R}^{2 n+1}$ (see [Whi12], pg. 113):

Corollary 2.1.18: $\quad$ On any manifold $M$ there exists a differentiable function with no degenerate critical points such that each $M^{a}$ is compact.

Corollary 2.1.19: $\quad$ Any differential manifold has the homotopy type of a $C W$-complex.

\subsection{Morse-Bott Theory}

Bott's extension of Morse Theory, which was first started in [Bot54], instead of analysing maps with no degenerate critical points, focuses on the study of the so-called non-degenerate critical manifolds and how theorems of Morse theory can be extended to functions with such collections of critical points. In this section, $M$ is a compact, Hausdorff, second countable finite dimensional manifold. More precisely, we have:

Definition 2.2.1: $\quad$ Let $f: M \rightarrow \mathbb{R}$ be a smooth function on $M$ and let $C$ be a smooth connected submanifold of the regular half space $\left.\left.M^{a}=f^{-1}(]-\infty, a\right]\right)$. Such a manifold is called a nondegenerate critical manifold of $f$ on $M^{a}$ if:

1. Each point of $C$ is a critical point of $f$;

2. For any $p \in C$, the nullspace of $H_{p} f$ is precisely the tangent space of $C$ at $p$.

In other words, on a non-degenerate critical manifold $C$, the matrix of the Hessian of $f$ in the direction of the normal to $C$ at a point $p \in C$, which will be denoted by $M_{p}^{\eta}$, is non-degenerate.

Notice that, if $C$ is simply a point $p$, then the conditions above imply that $p$ is a critical point where $H_{p} f$ is non-singular. Therefore the above concept generalises the definition of a nondegenerate critical point. We also consider a generalisation of Morse functions by

Definition 2.2.2: $\quad$ A smooth function $f: M \rightarrow \mathbb{R}$ on a smooth manifold $M$ is called a MorseBott function if the set of its critical points is the disjoint union of non-degenerate critical manifolds.

As an example of how Morse-Bott functions are much more general than Morse functions, we can see that now, the set of critical points of the height function on the horizontal torus, although with many non-degenerate critical points, is a union of non-degenerate critical manifolds. Also, the map $\left(x_{0}, \ldots, x_{n}\right) \rightarrow x_{n}^{2}$ on the $n$-sphere, which contains the equator of $S^{n}$ on the set of critical points, is a Morse-Bott function.

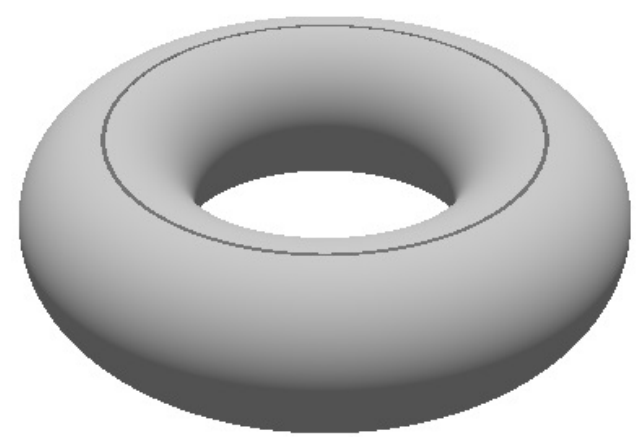


An immediate result which arises from the definition of non-degenerate critical manifold is:

Lemma 2.2.1: $\quad$ Let $C$ be a non-degenerate critical manifold of $f$. Then $f$ is constant in $C$.

This is immediate from the definition, since the restriction of $f$ to $C$ is a function with a derivative which constantly equals zero. Since $C$ is connected, the result follows.

We ask whether there is still the notion of index on a non-degenerate critical manifold, and we see that this is the case:

Lemma 2.2.2: $\quad$ Let $C$ be a non-degenerate critical manifold of $f$. Then the index of the Hessian of $f$ is constant on $C$.

To show the result above, we will use the following result which was proven by Hirsch and used in his own proof of the Morse Lemma:

Lemma 2.2.3: (Hirsch's Lemma) Let $A=\operatorname{diag}\left(a_{1}, \ldots, a_{m}\right)$ be a diagonal matrix with each $a_{i}= \pm 1$. Then there is a neighbourhood $U$ of $A$ in the space of $m \times m$ symmetric matrices (identified with $\mathbb{R}^{\frac{m(m+1)}{2}}$ ) and a smooth map $P: U \rightarrow G L_{m}(\mathbb{R})$ such that $P(A)=I_{m}$ and, for all $S \in U$, we have $P(S)^{t} \cdot S \cdot P(S)=A$.

Proof of Lemma 2.2.2: Let $f: M \rightarrow \mathbb{R}$ be a smooth function on a manifold $M$ of dimension $m$, and $C$ a non-degenerate critical manifold of $f$ of dimension $n$.

Replacing $f$ by $f-f(C)$, we assume, without loss of generality, that $f(C)=0$. Also, for each $p \in C$, we choose a neighbourhood $U$ of $p$ and a coordinate chart $\phi: U \rightarrow \mathbb{R}^{n} \times \mathbb{R}^{m}$ such that $\phi(p)=0$ and $\phi(U \cap C)=\mathbb{R}^{n} \times\{0\}$. Besides, let $h(x, y)=\left(f \circ \phi^{-1}\right)(x, y)$ and let $M_{x}^{\eta}$ be the matrix of the Hessian of $h$ in the direction normal to $\mathbb{R}^{n} \times\{0\}$ at $(x, 0)$, i.e:

$$
M_{x}^{\eta}=\left(\left.\frac{\partial^{2} h}{\partial y_{i} \partial y_{j}}\right|_{(x, 0)}\right)
$$

We see that $M_{x}^{\eta}$ depends smoothly of $x$ and, since $C$ is a non-degenerate critical manifold, then $M_{x}^{\eta}$ is non-degenerate, for all $x$. By composing the chart $\phi$ with a diffeomorphism of $\mathbb{R}^{n} \times \mathbb{R}^{m-n}$, which is constant in the first component and "well-behaved" in the second component, we assume that matrix $M_{0}^{\eta}$, which is the matrix with respect to the point $p$, is diagonal with the first $\lambda$ entries equal to -1 and the rest equal to 1 .

Using Hirsch's Lemma, we see there is a small neighbourhood $V$ of $0 \in \mathbb{R}^{n}$ and matrices $Q_{x}$ depending smoothly on $x \in V$, such that

$$
Q_{x}^{t} \cdot M_{x}^{\eta} \cdot Q_{x}=M_{0}^{\eta}
$$


Therefore, for any $i=n+1, \ldots, m-n$, we have

$$
\begin{aligned}
\left(Q_{x} y_{i}\right)^{t} M_{x}^{\eta}\left(Q_{x} y_{i}\right) & =y_{i}^{t} Q_{x}^{t} M_{x}^{\eta} Q_{x} y_{i} \\
& =y_{i}^{t} M_{0}^{\eta} y_{i} \\
& =\left.\frac{\partial^{2} h}{\partial y_{i}^{2}}\right|_{(0,0)}
\end{aligned}
$$

which is, by construction, -1 if $i=1, \ldots, \lambda$ and 1 if $i=\lambda+1, \ldots, m-n$. Using these ideas, we see that, writing the matrix $M_{x}^{\eta}$ on the basis $Q_{x}\left(y_{1}\right), \ldots, Q_{x}\left(y_{m-n}\right)$ we obtain precisely a diagonal matrix with $\lambda$ entries equal to -1 and the rest equals to 1 . Hence, $M_{x}^{\eta}$ also has index $k$, for all $x \in U$.

Thus the index of the hessian is locally constant in $C$ and therefore, since $C$ is connected, the index is constant in all of $C$.

Definition 2.2.3: $\quad$ Let $C$ be a non-degenerate critical manifold of a smooth function $f$. Then the common index of the Hessian of $f$ at $C$ is called the index of $C$ and denoted $\lambda_{f}(C)$.

By fixing a Riemannian structure on $M$ we see that, at each point $p \in C$, the bilinear form $H_{p} f$ uniquely determines a linear self-adjoint transformation $T_{p}$ on the tangent space of $M$ at $p$, by the formula

$$
<T_{p} X, Y>=H_{p} f(X, Y)
$$

These linear transformations combine to define a linear endomorphism, $T$, of the tangent space to $M$ along $C$. Since $C$ is a non-degenerate manifold, we know that the kernel of $T$ must be precisely the tangent space to $C$, and therefore, we think of $T$ as an automorphism of the normal bundle of $C$ in $M$.

Definition 2.2.4: $\quad$ The negative bundle, $\eta^{-}$, of $C$ is the subbundle of the normal bundle of $C$ in $M$ which is spanned by the negative eigenvectores of $T$. Thus the fiber of $\eta^{-}$at $p \in C$ is spanned by the normal vectors to $C$ at $p$, for which $T_{p} \cdot Y=\alpha Y, \alpha<0$. The fiber of $\eta^{-}$therefore has dimension $\lambda_{f}(C)$. If $\lambda_{f}(C)=0$, we set $\xi^{-}$equal to $C$.

Also, we define $\eta^{+}$to be the negative bundle of the function $-f$ along $C$. Clearly, the normal bundle $\eta$ on $C$ in $M$ is the direct sum $\eta=\eta^{+} \oplus \eta^{-}$.

Thus both bundles $\eta^{+}$and $\eta^{-}$are independent of the Riemannian structure used.

If, using classical Morse Theory, one constructs the halfspaces of a manifold by attaching cells, we can now construct such halfspaces by attaching a bundle. We define precisely what we mean by this.

Recall that if $\alpha: A \rightarrow Y$ is a map of a subset $A \subset X$ one forms the space $Y \cup_{\alpha} X$ by identifying $a \in A \subset X$ with $\alpha(a) \in Y$ in the disjoint union of $X$ and $Y$. Such attaching construction has the following properties:

1. The homotopy type of $Y \cup_{\alpha} X$ depends only on the homotopy type of $\alpha$. 

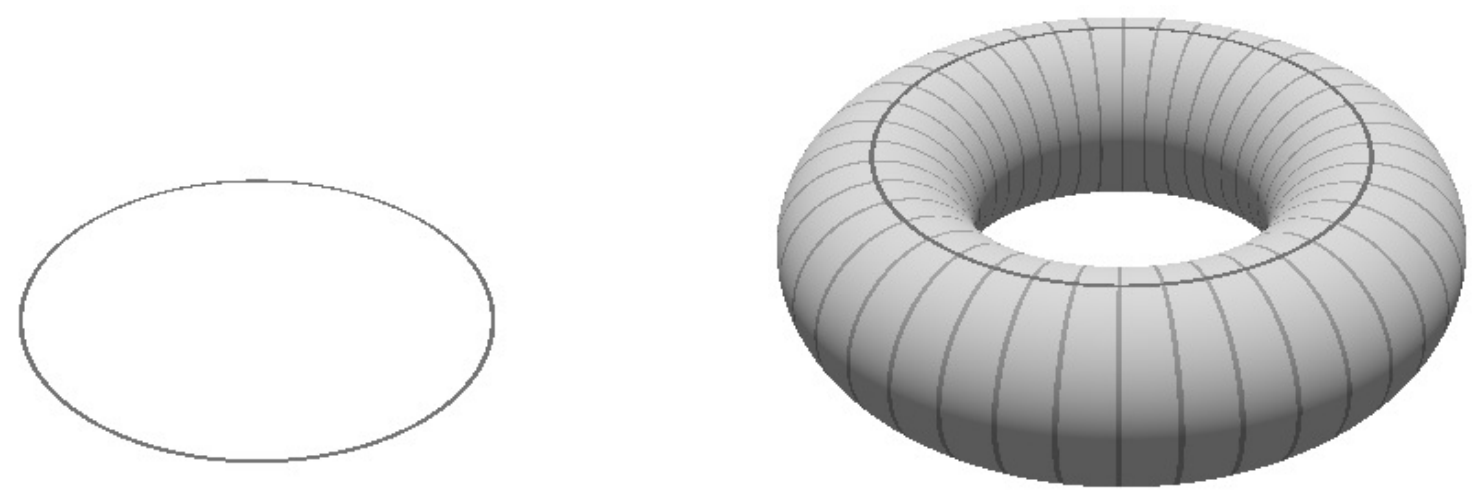

2. If $\left(X_{1}, A_{1}\right)$ is a deformation retract of $(X, A)$ and if $\alpha_{1}=\left.\alpha\right|_{A_{1}}$, then $Y \cup_{\alpha_{1}} X_{1}$ is of the same homotopy type as $Y \cup_{\alpha} X$.

We will use the above construction to define what it is to attach a bundle.

Definition 2.2.5: $\quad$ Let $\xi$ be an orthogonal n-vector bundle, $D_{\xi}$ be the sub-bundle formed by vectors of length less or equal to 1 and $S_{\xi}=\partial D_{\xi}$. Given a map $\alpha: S_{\xi} \rightarrow Y$, we form a space $Y \cup_{\alpha} \xi$ by identifying $x \in S_{\xi}$ with $\alpha(x) \in Y$ in the disjoint union of $Y$ and $D_{\xi}$. In this case we speak of $Y$ with $\xi$ attached, and if the function $\alpha$ is not specified, we denote it by $Y \cup \xi$. If $\xi$ is a 0-dimensional vector-bundle, $Y \cup \xi$ stands for the disjoint union of $Y$ with the base space of $\xi$.

Making precise the idea of generalisation of the results from Morse Theory, we get:

Theorem 2.2.4: $\quad$ Suppose that $M^{a} \subset M^{b}$ are two regular half-spaces of the function $f$ on the compact manifold $M$. Then

1. If $f$ has no critical points in the range $a \leq f \leq b$ then $M^{a}$ is a deformation retract of $M^{b}$.

2. If $f$ has a single non-degenerate manifold $C$ in the range $a \leq f \leq b$, then $M^{b}$ is of the same homotopy type as $M^{a}$ with the negative bundle of $f$ along $C$ attached:

$$
M^{b}=M^{a} \cup \eta^{-}
$$

The case when $C$ is a point $p$ is treated in section 2.1. This extension of the previous result is based on what was done in a neighbourhood of $p$ in the proof of Theorem 2.1.12, but in a normal neighbourhood of the non-degenerate manifold $C$. Now, on each fiber of such a neighbourhood, we have a non-degenerate critical point case.

To show the result above we use some notation and results from Riemannian Geometry. Precisely, we use the map exp.

Definition 2.2.6: Let $v \in T_{p} M$ be a tangent vector to $p \in M$, and suppose there exists a geodesic $\gamma:[0,1] \rightarrow M$ satisfying the conditions

$$
\gamma(0)=p, \quad \frac{d \gamma}{d t}(0)=v
$$

Then the point $\gamma(1) \in M$ is called the exponential of the tangent vector $v$, and it is denoted by $\exp _{p}(v)$. 
Lemma 2.2.5: If $M$ is a compact manifold, then the map exp is defined for all $p \in M$ and $v \in T_{p} M$.

The proof of the result above is included in Section 10 of [Mil63], and it guarantees that the map $\exp _{p}(v)$ is defined for all $p$, provided that $\|v\|$ is small enough. We have even more:

Lemma 2.2.6: For each $p \in M$, there exists a neighbourhood $W$ and a number $\varepsilon>0$, such that, for each $q \in W$, the map $\exp _{q}$ maps the open $\varepsilon$-ball in $T_{q} M$ diffeomorphically onto an open set $U_{q}$ which contains $W$.

Equipped with such tools, we move to the proof of the theorem:

Proof of Theorem 2.2.4: Item 1 of Theorem 2.2.4 was proved on Theorem 2.1.12. We now show that part 2 holds.

Without loss of generality, by replacing $f$ by $f-f(C)$, we may assume that $f(C)=0$ and that there is an $\varepsilon_{0}>0$, such that $f$ has no critical points in the range $\left(-\varepsilon_{0}, 0\right)$ and $\left(0,-\varepsilon_{0}\right)$. Under these conditions, it is sufficient to prove that $M^{\varepsilon}=M^{-\varepsilon} \cup \eta^{-}$, for some $0<\varepsilon<\varepsilon_{0}$.

Let us start with some notations. Recall that $\eta=\eta^{-} \oplus \eta^{+}$, and let $\pi: \eta \rightarrow \eta^{-}$be the natural projection. Let $\varphi: \eta \rightarrow \mathbb{R}$ be the map which associates each $X \in \eta$ to the value $\|X\|^{2}$, and let $\exp : \eta \rightarrow M$ be the exponential map. Also, let $f_{*}$ be function $f \circ \exp : \eta \rightarrow \mathbb{R}$.

If we look at $C$ included in $\eta$ as the zero section, we see that $\exp$ is a homeomorphism on a neighbourhood $V$ of $C$ and so it can be used to induce a Riemannian structure $(\cdot, \cdot)$ on this neighbourhood, i.e., for all $(p, U) \in V$

$$
\left.(X, Y)=<\exp _{*}(X), \exp _{*}(Y)\right)>
$$

Since $C$ is a non-degenerate critical manifold of $f$ we know that the function $f_{*}$ restricted to any fiber of $\eta$ has a non-degenerate critical point on 0 , which is of index $\lambda_{f}(C)$. More precisely, from the definitions of $\eta^{-}$, we see that the restriction of $f_{*}$ to any fiber of $\eta^{-}$has a non-degenerate maximum at 0 and that $f_{*}$ restricted to $\eta^{+}$has a non-degenerate minimum at 0 .

This implies that the function $\left(\nabla f_{*}, \nabla \varphi\right)$, which relates the direction in which $f_{*}$ increases to the size of the vectors in $\eta$, has 0 as a non-degenerate minimum when restricted to any fiber of $\eta^{+}$, and a non-degenerate maximum at 0 , when restricted to any fiber of $\eta^{-}$.

From the remarks above, we see that, if $\varepsilon>0$ is small enough, the set $f_{*} \leq \varepsilon$ on a fiber of $\eta^{+}$is star-shaped with respect to 0 , and therefore, linearly contractible.

Also, since $\left(\nabla f_{*}, \nabla \varphi\right)$ has a non-degenerate maximum at 0 , when restricted to any fiber of $\eta^{-}$, if we choose $\mu>0$ small enough, the gradient of $-f_{*}$ at points with $\varphi(X)=\mu$ points out the set $\varphi(X) \leq \mu$, on any fiber of $\eta^{-}$.

Now, define the set

$$
X_{\mu}^{\varepsilon}=\left\{X \in \eta: f_{*}(X) \leq \varepsilon ; \varphi \circ \pi(X) \leq \mu\right\}
$$

As a consequence of the remarks above, we can choose positive numbers $\varepsilon$ and $\mu$ such that: 
1. We have $\varepsilon<\varepsilon_{0}$;

2. The map exp is a homeomorphism on $X_{\mu}^{\varepsilon}$;

3. If $A_{\mu}^{\varepsilon} \subset X_{\mu}^{\varepsilon}$ is the subset of $X_{\mu}^{\varepsilon}$ on which $\varphi \circ \pi(X)=\mu$, then the pair $\left(X_{\mu}^{\varepsilon} \cap \eta^{-}, A_{\mu}^{\varepsilon} \cap \eta^{-}\right)$ is a deformation retract of $\left(X_{\mu}^{\varepsilon}, A_{\mu}^{\varepsilon}\right)$;

4. The gradient of $-f$ points out of the set $\exp \left(X_{\mu}^{\varepsilon}\right)$ at the points of $\exp \left(A_{\mu}^{\varepsilon}\right)$.

Let $\varepsilon$ and $\mu$ be chosen as described above and define $Y_{\mu}^{\varepsilon}=\overline{M^{\varepsilon} \backslash \exp \left(X_{\mu}^{\varepsilon}\right)}$.

From item (2), it is immediate that $M^{\varepsilon}=Y_{\mu}^{\varepsilon} \cup_{\alpha} X_{\mu}^{\varepsilon}$ with attaching map $\alpha=\left.\exp \right|_{A_{\mu}^{\varepsilon}}$. We will finish this proof by showing that $Y_{\mu}^{\varepsilon} \cup_{\alpha} X_{\mu}^{\varepsilon}$ is actually equivalent to $Y_{\mu}^{\varepsilon} \cup \eta^{-}$, and that $M^{-\varepsilon}$ is a deformation retract of $Y_{\mu}^{\varepsilon}$.

There is a clear homotopy equivalence (which is constructed through line paths) between the pairs $\left(D_{\eta^{-}}, S_{\eta^{-}}\right)$and $\left(X_{\mu}^{\varepsilon} \cap \eta^{-}, A_{\mu}^{\varepsilon} \cap \eta^{-}\right)$. Therefore, it follows directly from item (3) that $M^{\varepsilon}=Y_{\mu}^{\varepsilon} \cup \eta^{-}$.

Finally, from item (4), we can see that at the boundary points of $Y_{\mu}^{\varepsilon}$, the gradient $-\nabla f$ points inward to $Y_{\mu}^{\varepsilon}$. Furthermore, from item (1), we know there are no points critical points in the range $-\varepsilon \leq f<0$, and therefore, there are no points with $\nabla f=0$ on $Y_{\mu}^{\varepsilon}$. Using the same strategy we developed in Theorem 2.2.4 to show that there is a deformation retract between two half-spaces with no critical points between them, we can show that $M^{-\varepsilon}$ is a deformation retract of $Y_{\mu}^{\varepsilon}$. Precisely, we create a one parameter group of diffeomorphisms related to the gradient of $-f$ and, with this, construct the desired deformation retract.

Thus, we have shown that $M^{\varepsilon}$ is of the same homotopy type as $M^{-\varepsilon} \cup \eta^{-}$.

An immediate consequence of this theorem in homotopy is:

Corollary 2.2.7: $\quad$ Under the assumptions of item 2 of Theorem 2.2.4:

$$
M^{b}=M^{a} \cup e_{1} \cup e_{2} \cup \ldots
$$

where the cells $e_{i}, i=1,2, \ldots$, have dimension $\geq \lambda_{f}(C)$. In particular,

$$
\pi_{r}\left(M^{b}, M^{a}\right)=0 \quad \text { for } 0 \leq r<\lambda_{f}(C)
$$

Proof of Corollary 2.2.7: From item (2) of Theorem 2.2.4, we know that $M^{b}=M^{a} \cup_{\alpha} \eta^{-}$, for some map $\alpha$. Since $C$ is a closed submanifold of the compact manifold $M$, we know from the results of section 2.1 that $C$ is homotopy equivalent to a $C W$-complex. Without loss of generality, assume $C$ is a $\mathrm{CW}$-complex.

For each cell $e_{\alpha}^{k}$ in $C$, let $\phi_{\alpha}: D_{\alpha}^{k} \rightarrow C$ be its characteristic map and $\tilde{D}_{\alpha}$ the pre-image of $e_{\alpha}^{k}$ on the fibration $D_{\eta^{-}} \rightarrow C$. We can see there is a map

$$
\begin{aligned}
\tilde{\phi}_{\alpha}: D^{k} \times D^{\lambda_{f}(C)} & \rightarrow D_{\eta^{-}} \\
(x, v) & \rightarrow\left(\phi_{\alpha}(x), v\right)
\end{aligned}
$$


whose image is precisely $\tilde{D}_{\alpha}$ and which is a homeomorphism in $\operatorname{int}\left(D^{k}\right) \times D^{\lambda_{f}(C)}$, since $\phi_{\alpha}$ is a homeomorphism in the interior of $D^{k}$. It is simple to see there is a homeomorphism from $D^{k} \times D^{\lambda_{f}(C)}$ to $D^{k+\lambda_{f}(C)}$ which takes $\partial\left(D^{k}\right) \times \partial\left(D^{\lambda_{f}(C)}\right)$ to $\partial\left(D^{k+\lambda_{f}(C)}\right)$. Therefore, we think of the map $\tilde{\phi}_{\alpha}$ as the characteristic map of the $\left(k+\lambda_{f}(C)\right)$-cell $\tilde{D}_{\alpha}$.

This gives a CW-structure to $D_{\eta^{-}}$and, from the definition of attaching a bundle, we see $M^{a} \cup \eta^{-}$ as attaching the cells $\tilde{D}_{\alpha}$ to $M^{a}$ through the maps $\alpha \circ \tilde{\phi}_{\alpha}$.

Therefore the relation $M^{b}=M^{a} \cup \eta^{-}$yields that $M^{b}$ is obtained by $M^{a}$ by attaching cells of dimension $\geq \lambda_{f}(C)$, and therefore, $\pi_{r}\left(M^{b}, M^{a}\right)=0$, for all $0 \leq r<\lambda_{f}(C)$.

The restriction that $C$ is the only critical set of $f$ in the range from $a$ to $b$ is not essential. If all the critical sets are non-degenerate, they are necessarily finite in number because $M$ is compact, so if we denote them by $C^{i}, i=1, \ldots, s$, then Theorem 2.2 .4 is easily modified to yield the formula

$$
M^{b}=M^{a} \cup \eta_{1}^{-} \cup \cdots \cup \eta_{s}^{-}
$$

Corollary 2.2.8: Let $f$ be a smooth function on a compact manifold $M$. Assume that the critical set of $f$ consists entirely of non-degenerate critical manifolds. Let $M_{*}$ be the set on which $f$ takes its absolute minimum, and let $|f|$ denote the smallest index of the critical points of $f$ on $M \backslash M_{*}$. Then $M$ is obtained from $M_{*}$ by successively attaching cells of dimension no less than $|f|$. Thus

$$
\begin{array}{lr}
M=M_{*} \cup e_{1} \cup \cdots \cup e_{k} ; & \text { dim } e_{i} \geq|f| \\
\pi_{i}(M)=\pi_{i}\left(M_{*}\right) ; & \text { for all } i<|f|
\end{array}
$$

\subsection{Morse Theory on the Loop Space}

Morse had already started working with a generalisation of this theory to study the loop space of a manifold. The thing in question is that such a space is not a finite dimensional manifold, and therefore, some adaptations needed to be made. Throughout this section, let $M$ denote a connected finite dimensional Riemannian manifold of class $C^{\infty}$.

We begin with the extension of Morse Theory to the loop space of a manifold using the approach made by Bott in his paper [Bot59]. The motivation to study the loop space $\Omega M$ of a manifold comes mainly from the relation $\pi_{i}(\Omega M)=\pi_{i+1}(M)$ which gives us information about the homotopy groups of our manifold. Such space of maps is usually endowed of the compact-open topology, which is described by the metric

$$
d^{*}\left(c, c^{\prime}\right)=\max _{t \in[0,1]} \rho\left(c(t), c^{\prime}(t)\right)
$$

where $\rho$ is the metric on the manifold $M$.

However, instead of looking directly at $\Omega M$, it will suit best to study spaces which are homotopy equivalent to it, but have better properties for us to work with. Instead of studying the loops 
on a point of $M$ we look at the paths connecting two points of $M$. This choice of points on the manifold will be essential on the proof of Bott's Periodicity Theorem. Precisely, we have:

Definition 2.3.1: $\quad$ Let $\mu=(p, q)$, where $p$ and $q$ are arbitrary points of $M$. We define $\Omega_{\mu} M$ as the space of piecewise differentiable maps $c:[0,1] \rightarrow M$ on $M$, which are parametrized proportionally to arc length, take 0 to $p$ and 1 to $q$. We define the distance d between two points $c$ and $c^{\prime}$ in $\Omega_{\mu} M$ by:

$$
d\left(c, c^{\prime}\right)=\max _{t \in[0,1]}\left\{\rho\left(c(t), c^{\prime}(t)\right)\right\}+\left|J(c)-J\left(c^{\prime}\right)\right|
$$

where $\rho$ is the metric on the manifold $M$, and $J$ is the length function on $\Omega_{\mu} M$.

Recall that, since $M$ is a connected manifold, the fibers of the path fibration over $M$ are all homotopy equivalent. This implies that looking at loops on the basepoint (which would be the canonical fiber of the path fibration) is, from the homotopic point of view, the same as looking at paths from the basepoint to another point $p \in M$ (which would be the fiber over $p$ ). Therefore, the original loop space $\Omega M$ with the metric $d^{*}$ is homotopy equivalent to the space $\Omega_{\mu}^{*} M$ of paths between two given points, with the metric $d^{*}$. We use the notation $\Omega_{\mu}^{*} M$ to differentiate such space from the $\Omega_{\mu} M$ defined above, which is composed only of piece-wise differentiable maps.

There are many advantages in the definition above and of choosing the metric $d$, for instance, the length function becomes a continuous function on $\Omega_{\mu} M$ (which does not happen with the original topology). However, we have to see that we can work on such a space with this new metric without errors, that is, that $\left(\Omega_{\mu} M, d\right)$ is homotopy equivalent to $\left(\Omega_{\mu}^{*} M, d^{*}\right)$, which is the one we used to work with in homotopy theory.

Since $d \geq d^{*}$ we have a natural continuous map $i:\left(\Omega_{\mu} M, d\right) \rightarrow\left(\Omega_{\mu}^{*} M, d^{*}\right)$.

Theorem 2.3.1: $\quad$ This natural map $i$ is a homotopy equivalence.

Proof of Theorem 2.3.1: To facilitate notation, we denote by $\Omega$ the space $\Omega_{\mu} M$ endowed with the metric $d$ and by $\Omega^{*}$ the space $\Omega_{\mu}^{*} M$ with $d^{*}$.

The strategy we use to show that such spaces are equivalent is to decompose both of them in a countable amount of subspaces $\Omega_{k}$ and $\Omega_{k}^{*}$ in which we can explicitly show that the restriction of $i$ is homotopy equivalences. We then use this information we obtained in the smaller parts, to show that $i$ is indeed a homotopy equivalence between $\Omega$ and $\Omega^{*}$.

We use the well-known fact that every point on $M$, a Riemannian manifold, has an open neighbourhood $N$ which is "geodesically convex" in the sense that any two points of $N$ are joined by a unique minimal geodesic which lies completely within $N$ and depends differentiably on the endpoints.

Let $\left\{N_{\alpha}\right\}$ be a covering of $M$ by such geodesically convex open sets. For every continuous path $\gamma$, we know that $[0,1]$ can be covered by a finitely many open sets of the form $\gamma^{-1}\left(N_{\alpha}\right)$, since $[0,1]$ is compact. Then, we can define an integer $k>0$ such that $\left[\frac{j}{2^{k}}, \frac{j+1}{2^{k}}\right]$ is contained in some $\gamma^{-1}\left(N_{\alpha}\right)$, for all $i$. 
This motivates us to define, for all positive integer $k$, the set $\Omega_{k}^{*}$ of all continuous paths $\gamma$ from $p$ to $q$ such that the image of any subinterval $\left[\frac{j}{2^{k}}, \frac{j+1}{2^{k}}\right]$ by $\gamma$ is contained in one of the $N_{\alpha}$ of the covering.

We see from the remarks above that $\Omega^{*}$ is the union of the sequence

$$
\Omega_{1}^{*} \subset \Omega_{2}^{*} \subset \Omega_{3}^{*} \subset \ldots
$$

and it is simple to see that each $\Omega_{k}^{*}$ is open in $\Omega^{*}$.

Using the continuity of $i$, we can then produce corresponding open sets $\Omega_{k}=i^{-1}\left(\Omega_{k}^{*}\right)$ in $\Omega$ whose union is precisely $\Omega$.

What we do now is show that, for each $k$, the restriction $\left.i\right|_{\Omega_{k}}: \Omega_{k} \rightarrow \Omega_{k}^{*}$ is a homotopy equivalence, by constructing a homotopy inverse $h_{k}$ : for each $\gamma \in \Omega_{k}^{*}$, let $h_{k}(\gamma) \in \Omega_{k}$ be the broken geodesic which coincides with $\gamma$ when $t=\frac{j}{2^{k}}, j=0,1,2, \ldots, 2^{k}$, and in each subinterval $\left[\frac{j}{2^{k}}, \frac{j+1}{2^{k}}\right]$ is the minimal geodesic joining $\gamma\left(\frac{j}{2^{k}}\right)$ to $\gamma\left(\frac{j+1}{2^{k}}\right)$. This construction defines a function

$$
h_{k}: \Omega_{k}^{*} \rightarrow \Omega_{k}
$$

and it is not difficult to verify that $h$ is continuous.

Now we verify that $\left.i\right|_{\Omega_{k}} \circ h_{k}$ is homotopic to the identity map of $\Omega_{k}^{*}$, by the explicit construction of a homotopy $H_{u}: \Omega_{k}^{*} \rightarrow \Omega_{k}^{*}, u \in[0,1]$ setting for $\frac{j}{2^{k}} \leq u \leq \frac{j+1}{2^{k}}$ :

$$
\begin{aligned}
& \left.H_{u}(\gamma)\right|_{\left[0, \frac{j}{2^{k}}\right]}=\left.\left(\left.i\right|_{\Omega_{k}} \circ h_{k}\right)(\gamma)\right|_{\left[0, \frac{j}{2^{k}}\right]} \\
& \left.H_{u}(\gamma)\right|_{\left[\frac{j}{2^{k}}, u\right]}=\text { minimal geodesic from }\left(\left.i\right|_{\Omega_{k}} \circ h_{k}\right)(\gamma)\left(\frac{j}{2^{k}}\right) \text { to } \gamma(u) \\
& \left.H_{u}(\gamma)\right|_{[u, 1]}=\left.\gamma\right|_{[u, 1]}
\end{aligned}
$$

Then $H_{0}$ is the identity map of $\Omega_{k}^{*}$ and $H_{1}=\left.i\right|_{\Omega_{k}} \circ h$. It is easily verified that $H_{u}(\omega)$ is continuous as a function of both variables. Then $\left.i\right|_{\Omega_{k}} \circ h$ is homotopic to the identity map of $\Omega_{k}^{*}$. Similarly, we show that $\left.h \circ i\right|_{\Omega_{k}}$ is homotopic to the identity map of $\Omega_{k}$. This proves that $\left.i\right|_{\Omega_{k}}$ is a homotopy equivalence.

We also see that, since $\left.i\right|_{\Omega_{k}}$ and $h_{k}$ are homotopy inverses, for all $k$, in particular, both $h_{k}$ and $\left.h_{k+1}\right|_{\Omega_{k}^{*}}$ are homotopy inverses of $i_{\Omega_{k}}$, which implies, using Lemma 2.1.14, that $h_{k+1}$ can actually be seen to extend $h_{k+1}$. So we define $h: \Omega^{*} \rightarrow \Omega$, by setting $\left.h\right|_{\Omega_{k}^{*}}=h_{k}$, and this will be the homotopy inverse to $i$.

We conclude the proof using the following result, which can be seen as a generalisation of Whitehead's Theorem:

Lemma 2.3.2: $\quad$ Suppose that $X$ is the homotopy direct limit of $\left\{X_{i}\right\}$ and $Y$ is the homotopy direct limit of $\left\{Y_{i}\right\}$. Let $f: X \rightarrow Y$ be a map which carries each $X_{i}$ into $Y_{i}$ by a homotopy equivalence. Then $f$ itself is a homotopy equivalence. 
A proof of this result is seen in the Appendix of [Mil63].

Recall that a space $X$ is the homotopy direct limit of a sequence of subspaces $X_{1} \subset X_{2} \subset X_{3} \subset$ $\ldots$ if it is closely related to the space obtained by the telescoping construction on $X_{1}, X_{2}, \ldots$. Precisely, if

$$
X_{\Sigma}=\frac{\coprod_{n=1}^{\infty} X_{n} \times[n, n+1]}{\sim}
$$

where $\sim$ identifies $\left(x_{n}, n+1\right) \in X_{n} \times[n, n+1]$ with $\left(x_{n}, n+1\right) \in X_{n+1} \times[n+1, n+2]$, then $X$ is the direct limit of $X_{1} \subset X_{2} \subset X_{3} \subset \ldots$ if the projection map $p: X_{\Sigma} \rightarrow X$, which takes $(x, \tau) \rightarrow x$, is a homotopy equivalence.

We now give an idea of how to prove that both $\Omega$ and $\Omega^{*}$ are direct limits of the respective $\Omega_{k}$ and $\Omega_{k}^{*}$, which completes our proof.

Notice that each $\gamma \in \Omega$ lies in the interior of some $\Omega_{k}$. Since $\Omega$ is a metric space, it is paracompact and therefore, we can construct a partition of unity to construct a map $f: \Omega \rightarrow \mathbb{R}$ so that $f(\gamma) \geq k+1$ for all $\gamma \notin \Omega_{k}$, and $f(\gamma) \geq 1$ for all $\gamma$. The correspondence $\gamma \rightarrow(\gamma, f(\gamma))$ maps $\Omega$ homeomorphically onto a subset of

$$
\Omega_{\sum}=\frac{\coprod_{n=1}^{\infty} \Omega_{k} \times[k, k+1]}{\sim}
$$

which is clearly a deformation retract. Therefore $p$ is a homotopy equivalence and $\Omega$ is the homotopy direct limit of the $\Omega_{k}$ 's.

Using the same strategy as above, we can show that $\Omega^{*}$ is the homotopy direct limit of the $\Omega_{k}^{*}$ 's. Then, using Lemma 2.3.2, this completes the proof.

The theorem above guarantees us that we can study the homotopy groups of $\Omega_{\mu}(M)$ to understand the homotopy groups of the loop space of a manifold. The idea of applying Morse theory in such a space is basically to slice $\Omega_{\mu} M$ into the half-spaces of an interesting function (the energy map) and then show that each of those slices is homotopy equivalent to a half-space of a well-behaved function on a finite dimensional manifold. Therefore, we can apply Morse-Bott Theory to this new manifold, and through this homotopy equivalence, obtain results on the loop space. To do this procedure, we use some definitions and results from Calculus of Variations, which allows us to develop a sort of calculus on $\Omega_{\mu} M$. We now state some of the definitions and results which will be needed later. A more detailed account on this theory and the tools we use are found in Part III of [Mil63].

Definition 2.3.2: $\quad$ The tangent space of $\Omega_{\mu} M$ at a path $\omega$ is the vector space consisting of all piecewise smooth vector fields $W$ along $\omega$ for which $W(0)=W(1)=0$. This space is denoted $T_{\omega} \Omega_{\mu}$.

Definition 2.3.3: $\quad$ A variation of $\omega$ (keeping endpoints fixed) is a function

$$
\bar{\alpha}:(-\varepsilon, \varepsilon) \rightarrow \Omega_{\mu} M
$$


for some $\varepsilon>0$, such that $\bar{\alpha}(0)=\omega$ and there is a subdivision $0=t_{0}<t_{1}<\cdots<t_{k}=1$ so that the map

$$
\alpha:(-\varepsilon, \varepsilon) \times[0,1] \rightarrow M
$$

defined by $\alpha(u, t)=\bar{\alpha}(u)(t)$ is $C^{\infty}$ on each strip $(-\varepsilon, \varepsilon) \times\left[t_{i-1}, t_{i}\right], i=1, \ldots, k$.

More generally, if $(-\varepsilon, \varepsilon)$ is replaced by a neighbourhood $U$ of 0 in $\mathbb{R}$, then $\bar{\alpha}$ is called a $n$ parameter variation of $\omega$.

We also define the variation vector field associated to $\bar{\alpha}$ as the vector field $W$ along $\omega$ by

$$
W_{t}=\frac{d \bar{\alpha}}{d u}(0)_{t}=\frac{\partial \alpha}{\partial u}(0, t) \in T_{\omega} \Omega_{\mu} M
$$

It is natural to ask whether given a vector field $W$ along $\omega$ we can find a variation $\bar{\alpha}$ satisfying $\bar{\alpha}(0)=\omega$ and $\frac{d \bar{\alpha}}{d u}(0)=W$. This is true and such a variation is in fact very intuitive. We take for instance

$$
\bar{\alpha}(u)(t)=\exp _{\omega(t)}\left(u W_{t}\right)
$$

Definition 2.3.4: A path $\omega$ is a critical path for a function $F: \Omega_{\mu} M \rightarrow \mathbb{R}$ if and only if $\left.\frac{d F(\bar{\alpha}(u))}{d u}\right|_{u=0}$ is zero for every variation $\bar{\alpha}$ of $\omega$.

Now that we have the tools to think about "critical points" on $\Omega_{\mu} M$, we introduce the function central to how we look at the loop space:

Definition 2.3.5: Given a curve $\omega \in \Omega_{\mu} M$, and numbers $0 \leq a \leq b \leq 1$, we define the energy of $\omega$ from $a$ to $b$ as

$$
E_{a}^{b}(\omega)=\int_{a}^{b}\left\|\frac{d \omega}{d t}\right\|^{2} d t
$$

If $\mu=(p, q)$, we define the energy function on $\Omega_{\mu} M$ as the map

$$
\begin{aligned}
E: \Omega_{\mu} M & \rightarrow \mathbb{R} \\
\omega & \rightarrow E_{0}^{1}(\omega)
\end{aligned}
$$

We see that the energy map is actually closely related to the arc-length function on the loop space. We will show next that they even have the same critical paths. The main difference we would like to point out, and which makes it much more useful to us, is that the energy map is smooth and this is why we choose to work with it.

We now try to understand precisely what are the critical paths of $E$. First, let's set some notation: let $\bar{\alpha}:(-\varepsilon, \varepsilon) \rightarrow \Omega_{\mu} M$ be a variation of $\omega$; set $W_{t}=\frac{\partial \alpha}{\partial u}(0, t)$ the associated variation vector field; $V_{t}=\frac{d \omega}{d t}$ the velocity vector of $\omega ; A_{t}=\frac{D}{d t} \frac{d \omega}{d t}$ the acceleration vector of $\omega ;$ and $\Delta_{t} V=V_{t_{+}}-V_{t_{-}}$ 
the discontinuity in the velocity vector at $t$, where $0<t<1$ (which is non-zero only in a finite number of values of $t$ ).

Theorem 2.3.3: $\quad$ (First variation formula)

$$
\left.\frac{1}{2} \frac{d E(\bar{\alpha}(u))}{d u}\right|_{u=0}=-\sum_{t}<W_{t}, \Delta_{t} V>-\int_{0}^{1}<W_{t}, A_{t}>d t
$$

The proof of this result consists of computations using the nice properties of Riemannian manifolds and calculus of variations, and is found in Mil63] (Theorem 12.2).

Corollary 2.3.4: $\quad$ The critical paths of the energy map on $\Omega_{\mu} M$ are precisely the geodesics and its minimum points are the minimal geodesics from $p$ to $q$.

Proof of Corollary 2.3.4: Any geodesic is a critical path since, by the definition, $A_{t}=0$ and $\Delta_{t} V=0$ for all $t$.

On the other hand, let $\omega$ be a critical path of $E$. We choose a variation of $\omega$ such that $W_{t}=$ $f(t) A(t)$, where the function $f(t)$ is positive except that it vanishes on $t_{i}$. Since $\omega$ is a critical path then

$$
0=\frac{1}{2} \frac{d E}{d u}(0)=-\int_{0}^{1} f(t)<A_{t}, A_{t}>d t=-\int_{0}^{1} f(t)\left\|A_{t}\right\|^{2} d t
$$

Since we chose $f$ to be positive $] t_{i}, t_{i+1}\left[\right.$, the relation above implies that $A_{t}=0$ for all $\left.t \in\right] t_{i}, t_{i+1}[$ which, by continuity, implies that $A_{t}=0$, for all $t$. Then each $\left.\omega\right|_{t_{i}, t_{i+1}[}$ is a geodesic.

We can now choose another variation of $\omega$ which satisfies $W\left(t_{i}\right)=\Delta_{t_{i}} V$. Since $\omega$ is a critical path, we have

$$
0=\frac{1}{2} \frac{d E}{d u}(0)=-\sum_{t}<\Delta_{t} V, \Delta_{t} V>=-\sum_{t}\left\|\Delta_{t} V\right\|^{2}
$$

which only happens if all $\Delta V_{t_{i}}=0$. This guarantees us that $\omega$ is differentiable at all points $t$, and therefore, from the uniqueness theorem for differential equations, $\omega$ is an unbroken geodesic.

For the second statement, we establish a relation between the energy of a path $E(\omega)$ and its length $L(\omega)$, using Schwarz's inequality

$$
\left(\int_{0}^{1} f g d t\right)^{2} \leq\left(\int_{0}^{1} f^{2} d t\right)\left(\int_{0}^{1} g^{2} d t\right)
$$

When applied to $f=1$ and $g=\left\|\frac{d \omega}{d t}\right\|$, it yields

$$
L(\omega)^{2} \leq E(\omega)
$$

where equality holds if and only if $g$ is constant; that is if and only if $\left\|\frac{d \omega}{d t}\right\|=0$. 
When $\gamma$ is a minimal geodesic from $p$ to $q$, we have

$$
E(\gamma)=L(\gamma)^{2} \leq L(\omega)^{2} \leq E(\omega)
$$

We know that the equality $L(\gamma)^{2}=L(\omega)^{2}$ will only hold if $\omega$ is also a minimal geodesic from $p$ to $q$ and that $L(\omega)^{2}=E(\omega)$ if and only if $g$ is constant, i.e. if $\omega$ is parametrized proportionally to arc-length. Therefore, $E(\gamma)<E(\omega)$, unless $\omega$ is also a minimal geodesic.

Since we want to undertake a procedure similar to what was developed on finite dimensional manifolds with Morse Theory on the loop space, it will be useful to discuss what would be the hessian of the energy function, so we can think of indices of the critical paths. So what we want to do now is to define, for each geodesic $\gamma$, a symmetric bilinear map $E_{* *}: T_{\gamma} \Omega_{\mu} \times T_{\gamma} \Omega_{\mu} \rightarrow \mathbb{R}$ with nice properties, similar to the usual hessian of a map. It will come in hand to use the following result on 2-parameter variations of a geodesic:

Theorem 2.3.5: (Second Variation Formula) Let $\bar{\alpha}: U \rightarrow \Omega_{\mu} M$ be a 2-parameter variation of the geodesic $\gamma$ with variation vector fields $W_{i}=\frac{\partial \bar{\alpha}}{\partial u_{i}}(0,0) \in T_{\gamma} \Omega_{\mu} M$, for $i=1,2$, then

$$
\frac{1}{2} \frac{\partial^{2} E}{\partial u_{1} \partial u_{2}}(0,0)=-\sum_{t}\left\langle W_{2}(t), \Delta_{t} \frac{D W_{1}}{d t}\right\rangle-\int_{0}^{1}\left\langle W_{2}, \frac{D^{2} W_{1}}{d t^{2}}+R\left(V, W_{1}\right) V\right\rangle d t
$$

where $R$ denotes the curvature tensor on $M, V$ denotes the velocity vector field of $\gamma$ and $\Delta_{t} \frac{D W_{1}}{d t}=$ $\frac{D W_{1}}{d t}\left(t^{+}\right)-\frac{D W_{1}}{d t}\left(t^{-}\right)$, which is only non-zero in finitely many points in $] 0,1[$.

The proof of this result consists of computations using the nice properties of a Riemannian manifold and calculus of variations, and can be found in [Mil63] (Theorem 13.1).

Now we define $E_{* *}: T_{\omega} \Omega_{\gamma} M \times T_{\omega} \Omega_{\gamma} M \rightarrow \mathbb{R}$ as follows: given two vector fields $W_{1}, W_{2} \in T_{\omega} \Omega_{\gamma} M$ we choose a 2-parameter variation $\bar{\alpha}: U \rightarrow \Omega_{\mu} M$ where $U$ is a neighbourhood of $(0,0)$ in $\mathbb{R}^{2}$, so that

$$
\bar{\alpha}(0,0)=\gamma, \quad \frac{\partial \alpha}{\partial u_{1}}(0,0)=W_{1}, \quad \frac{\partial \alpha}{\partial u_{2}}(0,0)=W_{2}
$$

We define the Hessian $E_{* *}\left(W_{1}, W_{2}\right)$ as the second partial derivative

$$
\left.\frac{\partial^{2} E\left(\bar{\alpha}\left(u_{1}, u_{2}\right)\right)}{\partial u_{1} \partial u_{2}}\right|_{(0,0)}
$$

which we denote briefly as $\frac{\partial^{2} E}{\partial u_{1} \partial u_{2}}(0,0)$.

From the Second Variation Formula, we see that $E_{* *}\left(W_{1}, W_{2}\right)=\frac{\partial^{2} E}{\partial u_{1} \partial u_{2}}(0,0)$ depends only on the variation vector fields $W_{1}$ and $W_{2}$, and also that it is bilinear. Using the fact that $\frac{\partial^{2} E}{\partial u_{1} \partial u_{2}}=\frac{\partial^{2} E}{\partial u_{2} \partial u_{1}}$, we see that $E_{* *}$ is indeed a symmetric bilinear function. From this hessian, we define the index of a geodesic: 
Definition 2.3.6: $\quad$ The index $\lambda$ of a geodesic $\gamma \in \Omega_{\mu} M$ is defined as the maximum dimension of a subspace in which the hessian $E_{* *}$ in $\gamma$ is negative definite.

Lemma 2.3.6: If $\gamma$ is a minimal geodesic from $p$ to $q$ then the bilinear pairing $E_{* *}$ is positive semi-definite. Hence, the index $\lambda$ of $E_{* *}$ is zero.

Proof of Lemma 2.3.6: Fix an arbitrary vector field $W \in T_{\gamma} \Omega_{\mu} M$ and let $\bar{\alpha}:(-\varepsilon, \varepsilon) \rightarrow \Omega_{\mu} M$ be a variation of $\gamma$ with variation vector field equals to $W$.

We will show that for any such $W$, we have $E_{* *}(W) \geq 0$. To do that, consider the following 2-parameter variation:

$$
\bar{\beta}\left(u_{1}, u_{2}\right)=\bar{\alpha}\left(u_{1}+u_{2}\right)
$$

Then $\frac{\partial \bar{\beta}}{\partial u_{i}}=\frac{d \bar{\alpha}}{d u}(0)$ and

$$
E_{* *}(W, W)=\frac{\partial^{2} E \circ \bar{\beta}}{\partial u_{1} \partial u_{2}}(0,0)=\frac{d^{2} E \circ \bar{\alpha}}{d u^{2}}(0)
$$

Since we have proven that the minimal geodesics are the minimal points of the energy function, we know that $E(\bar{\alpha}(u)) \geq E(\bar{\alpha}(0))$, which implies that $\frac{d^{2} E \circ \bar{\alpha}}{d u^{2}}(0) \geq 0$ as required.

This is compatible with the results we had in the original Morse theory, when we showed that minimum points always had index zero. The last point we now study on our analogies is to determine conditions of when a critical path of $E$ is non-degenerate, i.e, when the hessian $E_{* *}$ is non-degenerate.

Definition 2.3.7: $\quad$ A vector field $J$ along a geodesic $\gamma$ is called a Jacobi field if there exists a variation $\bar{\alpha}$ (not necessarily keeping endpoints fixed) such that each $\bar{\alpha}(u)$ is a geodesic and

$$
\left.\frac{d \bar{\alpha}}{d u}\right|_{0}=J
$$

The totality of such vector fields along $\gamma$ forms a vector space $J_{\gamma}$ over the real numbers.

Two points $p, q$ in $\gamma$ are called conjugate if there exists a nontrivial Jacobi field along $\gamma$ which vanishes both at $p$ and $q$. The multiplicity of $p$ and $q$ as conjugate points is equal to the dimension of the vector space consisting of all such Jacobi fields.

Simple computations yield the following:

Lemma 2.3.7: $\quad$ A vector field $J$ along a geodesic $\gamma$ is a Jacobi field if and only if it satisfies the Jacobi differential equation

$$
\frac{D^{2} J}{d t^{2}}+R(V, J) V=0
$$

where $V=\frac{d \gamma}{d t}$.

Since the Jacobi equation is a linear, second order differential equation, by writing it in terms of a basis of $n$ orthonormal vector fields in $T_{\gamma} \Omega_{\mu} M$, we see that it has $2 n$ linearly independent 
solutions which are $C^{\infty}$ and which are completely determined by its initial conditions $J(0)$ and $\frac{D J}{d t}(0)$. The fact that the vector space $J_{\gamma}$ has finite dimension turns out to be an interesting result considering:

Theorem 2.3.8: $\quad$ A vector field $W_{1} \in T_{\gamma} \Omega_{\mu} M$ belongs to the null space of $E_{* *}$ if and only if $W_{1}$ is a Jacobi field. Hence $E_{* *}$ is degenerate if and only if the end points $p$ and $q$ are conjugate along $\gamma$. The nullity of $E_{* *}$ is equal to the multiplicity of $p$ and $q$ as conjugate points.

With this result, which is proved in Chapter 14 of [Mil63], we see that the nullity of $E_{* *}$ is always finite, for any geodesic on $\Omega_{\mu} M$.

Theorem 2.3.9: $\quad$ (Morse Index Theorem) The index $\lambda$ of $E_{* *}$ is equal to the number of points $\gamma(t)$, with $0<t<1$, such that $\gamma(t)$ is conjugate to $\gamma(0)$ along $\gamma$; each such conjugate point being counted with its multiplicity. This index $\lambda$ is always finite.

We prove this result by showing that $T_{\gamma} \Omega_{\mu} M$ can be split as a direct sum of two spaces, and that in one of those the restriction of $E_{* *}$ is positive definite. Therefore, to prove the result, it is enough to study the other subspace, which turns out to be much easier to work with.

We know that each point $\gamma(t)$ is contained in an open set $U$, such that any two points in $U$ are joined by a unique minimal geodesic which depends smoothly on the endpoints. Using the same compactity argument used in the proof of Theorem 2.3.1. we can find a partition $0=t_{0}<t_{1}<\cdots<t_{k}=1$, so that each segment $\left.\gamma\right|_{\left[t_{i}, t_{i+1}\right]}$ is contained in one such neighbourhood $U$ and so that each $\left.\gamma\right|_{\left[t_{i}, t_{i+1}\right]}$ is minimal.

We define the subspace of $T_{\gamma} \Omega_{\mu} M$ which is central to the proof: let $T_{\gamma} \Omega_{\mu}\left(t_{0}, \ldots, t_{k}\right)$ be the vector space consisting of all vector fields $W$ along $\gamma$ such that $W$ vanishes at the endpoints $t=0$ and $t=1$, and such that $\left.W\right|_{\left[t_{i}, t_{i+1}\right]}$ is a Jacobi field along $\left.\gamma\right|_{\left[t_{i}, t_{i+1}\right]}$, for each $i$. This makes $T_{\gamma} \Omega_{\mu}\left(t_{0}, \ldots, t_{k}\right)$ a finite dimensional vector space consisting of broken Jacobi fields along $\gamma$.

Lemma 2.3.10: $\quad$ The vector space $T_{\gamma} \Omega_{\mu} M$ splits as a direct sum $T_{\gamma} \Omega_{\mu}\left(t_{0}, \ldots, t_{k}\right) \oplus T$, in which these spaces are perpendicular with respect to the inner product $E_{* *}$ and such that the restriction of $E_{* *}$ to $T$ is positive definite.

Proof of Lemma 2.3.10: Let $T \subset T_{\gamma} \Omega_{\mu}$ be the space of all vector fields $W$ along $\gamma$ satisfying $W\left(t_{0}\right)=W\left(t_{1}\right)=\cdots=W\left(t_{k}\right)=0$.

By choosing a partition $0=t_{0}<\cdots<t_{k}=1$ in the way described above, we can see there is a unique Jacobi field along each $\left.\gamma\right|_{\left[t_{i}, t_{i+1}\right]}$, which equals $W$ at the endpoints $t_{i}$ and $t_{i+1}$. Using this tool, we can uniquely construct a broken Jacobi field $J$ along $\gamma$, such that $J\left(t_{i}\right)=W\left(t_{i}\right)$ for each $i$. Clearly $W-J$ belongs to $T$. Therefore, the subspaces $T_{\gamma} \Omega_{\mu}\left(t_{0}, \ldots, t_{k}\right)$ and $T$ generate $T_{\gamma} \Omega_{\mu} M$ and the only vector field they have in common is the zero.

To see that these spaces are orthogonal regarding $E_{* *}$, we compute $E_{* *}\left(W_{1}, W_{2}\right)$ using the Second Variation Formula (Theorem 2.3.5), with $W_{1} \in T_{\gamma} \Omega_{\mu}\left(t_{0}, \ldots, t_{k}\right)$ and $W_{2} \in T$ :

$$
\frac{1}{2} E_{* *}\left(W_{1}, W_{2}\right)=-\sum_{t}\left\langle W_{2}(t), \Delta_{t} \frac{D W_{1}}{d t}\right\rangle-\int_{0}^{1}\left\langle W_{2}, 0\right\rangle d t=0
$$


Since $W_{2}$ is zero precisely on the points where $\Delta_{t} \frac{D W_{1}}{d t}$ could be not null, we see that the above expression is zero, as required.

We will finish by showing that $E_{* *}$ is positive definite on $T$ : using a procedure analogous to what we did in 2.3.6. We compute $E_{* *}(W, W)$ from the second derivative $\frac{d^{2} E \circ \bar{\alpha}}{d u^{2}}(0)$, where $\bar{\alpha}$ is a variation of $\gamma$ with variation vector field $\frac{d \bar{\alpha}}{d t}(0)=W$. To do this procedure on a $W \in T$, we choose $\bar{\alpha}$ in a way as to leave the points $\gamma\left(t_{0}\right), \ldots, \gamma\left(t_{k}\right)$ fixed. Since the restriction $\left.\gamma\right|_{\left[t_{i}, t_{i+1}\right]}$ is a minimal geodesic, for all $i$, then the energy $E(\bar{\alpha}(0)) \leq E(\bar{\alpha})(u)$, for all $u$. This implies that the second derivative evaluated at $u=0$ must be non-negative.

Finally, we see that the only $W \in T$ with $E_{* *}(W, W)=0$ is the zero vector field. Let $W \in T$ under such conditions, we will show that it belongs to the null space of $E_{* *}$. We know that $E_{* *}\left(W, W_{2}\right)=0$ for all $W_{2} \in T_{\gamma} \Omega_{\mu}\left(t_{0}, \ldots, t_{k}\right)$. Now let $W_{2} \in T$, then for all $c>0$, the vector field $W-c W_{2} \in T$ and then, since $E_{* *}$ is bilinear and symmetric,

$$
0 \leq E_{* *}\left(W-c W_{2}, W-c W_{2}\right)=-2 c E_{* *}\left(W, W_{2}\right)+c^{2} E_{* *}\left(W_{2}, W_{2}\right)=-2 c E_{* *}\left(W, W_{2}\right)
$$

which implies that $E_{* *}\left(W, W_{2}\right)=0$. Hence, $W$ must be a Jacobi field, and therefore is zero.

Corollary 2.3.11: The index (or the nullity) of $E_{* *}$ is equal to the index (or nullity) of $E_{* *}$ restricted to the space $T_{\gamma} \Omega\left(t_{0}, t_{1}, \ldots, t_{k}\right)$ of broken Jacobi fields. In particular, since $T_{\gamma} \Omega\left(t_{0}, t_{1}, \ldots, t_{k}\right)$ is a finite dimensional vector space, the index $\lambda$ is always finite.

With this in hand, we prove the Morse Lemma, knowing that we only need to look for the restriction of $E_{* *}$ :

Proof of Theorem 2.3.9: The idea of this proof is to look at the restrictions $\gamma_{\tau}=\left.\gamma\right|_{[0, \tau]}$, with $\tau$ varying in $[0,1]$ and to study what happens to the index $\lambda(\tau)$ of the hessian $E_{* *}^{\tau}$ associated to the geodesic $\gamma_{\tau}$. Precisely, we show that the index $\lambda(\tau)$ changes only when $\gamma(\tau)$ passes a conjugate point to $\gamma(0)$.

First, notice that for small values of $\tau$, we know that $\gamma_{\tau}$ is a minimal geodesic, and therefore $\lambda(\tau)=0$. Also if $\tau<\tau^{\prime}$, then $\lambda(\tau) \leq \lambda\left(\tau^{\prime}\right)$, since vector fields along $\gamma_{\tau}$ which vanish in $\gamma(0)$ and $\gamma(\tau)$ can be extended to one along $\gamma_{\tau^{\prime}}$ by setting it to be identically zero between $\gamma(\tau)$ and $\gamma\left(\tau^{\prime}\right)$. Then we create a $\lambda(\tau)$ dimensional subspace along $\gamma_{\tau^{\prime}}$ in which $E_{* *}^{\tau^{\prime}}$ is negative definite, which implies $\lambda(\tau) \leq \lambda\left(\tau^{\prime}\right)$.

We now show that $\lambda(\tau)$ is a staircase function, i.e. a map which is constant but in a finite amount of points in which it is discontinuous. Moreover, we see that such discontinuity points of $\lambda$ are precisely the ones in which $\gamma(\tau)$ is a conjugate point to $\gamma(0)$.

We showed in Lemma 2.3.10 that the index of the geodesic $\gamma=\gamma_{1}$ is the index of the quadratic form induced by $E_{* *}$ on the finite dimensional vector space $T_{\gamma} \Omega\left(t_{0}, \ldots, t_{k}\right)$. If we want to reproduce this argument on an arbitrary $\gamma_{\tau}$, we can use the partition we had before to form one in $[0, \tau]$ : if $t_{i}<\tau \leq t_{i+1}$, we can take $0=t_{0}<t_{1}<\cdots<t_{i}<\tau$. Then, from Lemma 2.3.10, we know the index $\lambda(\tau)$ is the index of a quadratic form $H_{\tau}$ induced on $T_{\gamma_{\tau}} \Omega\left(t_{0}, \ldots, t_{i}, \tau\right)$. Since any such Jacobi fields vanishes on $\gamma(0)$ and $\gamma(\tau)$ and is uniquely determined by its values on the 
break points $\gamma\left(t_{k}\right)$, we see that this space is isomorphic to the direct sum

$$
\sum=T_{\gamma\left(t_{1}\right)} M \oplus T_{\gamma\left(t_{2}\right)} M \oplus \cdots \oplus T_{\gamma\left(t_{i}\right)} M
$$

Notice that $\sum$ is independent of $\tau$, for $t_{i}<\tau \leq t_{i+1}$.

By definition, we know there is a subspace $\mathscr{V} \subset \sum$ of dimension $\lambda(\tau)$, in which the restriction of $H_{\tau}$ is negative definite. Since clearly $H_{\tau}$ on $\sum$ varies continuously with $\tau$, we know that, for any $\tau^{\prime}$ sufficiently close to $\tau$, the quadratic form $H_{\tau^{\prime}}$ will also be negative definite on $\mathscr{V}$ and therefore $\lambda\left(\tau^{\prime}\right) \geq \lambda(\tau)$. On the other hand, since $\lambda$ is monotone, for small $\varepsilon>0$, we have $\lambda(\tau-\varepsilon)=\lambda(\tau)$. This shows us that $\lambda$ is continuous and locally constant from the left.

What is left to see is that the discontinuity points of $\lambda$ are precisely the points which are conjugate to $\gamma(0)$ and show that they increase the value of the index by their multiplicity. Explicitly, we show that for all $\tau$ and sufficiently small $\varepsilon>0$ we have

$$
\lambda(\tau+\varepsilon)=\lambda(\tau)+\nu
$$

where $\nu$ is the nullity of the hessian $E_{* *}^{\tau}$. Therefore, $\lambda$ jumps on the conjugate points to $\gamma(0)$.

Looking at the form $H_{\tau}$ on the space $\sum$ described above, we see that $H_{\tau}$ is positive definite on a space $\mathscr{V}^{\prime}$ of dimension $\operatorname{dim} \sum-\lambda(\tau)-\nu=i \cdot n-\lambda(\tau)-\nu$. Since $H_{\tau}$ varies continuously with $\tau$, for sufficiently small $\varepsilon>0$, the quadratic form $H_{\tau+\varepsilon}$ will also be positive definite on $\mathscr{V}^{\prime}$ and therefore

$$
\lambda(\tau+\varepsilon) \leq \operatorname{dim} \sum-\operatorname{dim} \mathscr{V}^{\prime}=\lambda(\tau)+\nu
$$

To show the other inequality, we create $\lambda(\tau)+\nu$ linearly independent vector fields along $\gamma_{\tau+\varepsilon}$, defining a subspace on which $E_{* *}^{\tau+\varepsilon}$ is negative definite: let $W_{1}, \ldots, W_{\lambda(\tau)}$ be $\lambda(\tau)$ linearly independent vector fields in $T_{\gamma_{\tau}} \Omega\left(t_{0}, \ldots, t_{i}, \tau\right)$, on which the matrix $\left(E_{* *}^{\tau}\left(W_{i}, W_{j}\right)\right)_{i j}$ is negative definite. Also, let $J_{1}, \ldots, J_{\nu}$ be $\nu$ linearly independent Jacobi fields along $\gamma_{\tau}$ vanishing at the endpoints. Notice that the $\nu$ vectors

$$
\frac{D J_{h}}{d t}(\tau) \in T_{\gamma(\tau)} M
$$

are linearly independent. Hence, it is possible to choose $\nu$ vector fields along $\gamma_{\tau+\varepsilon}$, vanishing at the endpoints of $\gamma_{\tau+\varepsilon}$ satisfying:

$$
\left.\left\langle\frac{D J_{h}}{d t}(\tau), X_{k}(\tau)\right)\right\rangle=\delta_{h k}
$$

We extend the vector fields $W_{i}$ and $J_{h}$ over $\gamma_{\tau+\varepsilon}$ by setting these fields equal to 0 for $\tau \leq t \leq \tau+\varepsilon$. Using the Second Variation Formula, we see that

$$
\begin{aligned}
& E_{* *}^{\tau+\varepsilon}\left(J_{h}, W_{i}\right)=0 \\
& E_{* *}^{\tau+\varepsilon}\left(J_{h}, X_{k}\right)=2 \delta_{h k}
\end{aligned}
$$


Now, for a small $c>0$, consider the $\lambda(\tau)+\nu$ vector fields along $\gamma_{\tau+\varepsilon}$

$$
W_{1}, \ldots, W_{\lambda(\tau)}, c^{-1} J_{1}-c X_{1}, \ldots, c^{-1} J_{\nu}-c X_{\nu}
$$

Notice that the matrix of the restriction of $E_{* *}^{\tau+\varepsilon}$ in this base is

$$
\left(\begin{array}{c:c}
\left(E_{* *}^{\tau}\left(W_{i}, W_{j}\right)\right)_{i j} & c A \\
\hdashline c A^{t} & -4 I+c^{2} B
\end{array}\right)
$$

for some matrices $A$ and $B$. Then, for a sufficiently small $c$, this matrix will be negative definite which implies that $\lambda(\tau+\varepsilon) \geq \lambda(\tau)+\nu$. Finally, we conclude that $\lambda(\tau+\varepsilon)=\lambda(\tau)+\nu$. This completes the proof.

So much for this incursion into calculus of variations, we now turn our attention to our original motivation: to "slice" the loop space $\Omega_{\mu} M$ into pieces which are homotopy equivalent to a finite dimensional CW-complex.

If $c$ is a real number, the subset of $\Omega_{\mu} M$, on which the energy $E \leq c$, will be denoted by $\Omega_{\mu}^{c}$, and referred to as a half space of $\Omega_{\mu} M$. What we will show is that every such a half space has the same homotopy type of a finite dimensional manifold.

First, let $i n t \Omega_{\mu}^{c}=E^{-1}[0, c)$ and, for each partition $0=t_{0}<t_{1}<\cdots<t_{k}=1$, let $\Omega\left(t_{0}, t_{1}, \ldots, t_{k}\right)$ be the space of broken geodesics (i.e. paths $\omega$ where $\left.\omega\right|_{\left[t_{i}, t_{i+1}\right]}$ is a geodesic for all $i$ ) from $p$ to $q$. We also define

$$
\begin{aligned}
& \Omega\left(t_{0}, t_{1}, \ldots, t_{k}\right)^{c}=\Omega_{\mu}^{c} \cap \Omega\left(t_{0}, t_{1}, \ldots, t_{k}\right) \\
& \operatorname{int} \Omega\left(t_{0}, t_{1}, \ldots, t_{k}\right)^{c}=\operatorname{int} \Omega_{\mu}^{c} \cap \Omega\left(t_{0}, t_{1}, \ldots, t_{k}\right)
\end{aligned}
$$

Lemma 2.3.12: $\quad$ Let $M$ be a complete Riemannian manifold (not necessarily compact). For each $c>0$ such that $\Omega_{\mu}^{c} \neq \emptyset$, there is a partition $0=t_{0}<t_{1}<\cdots<t_{k}=1$ such that int $\Omega\left(t_{0}, \ldots, t_{k}\right)^{c}$ can be given a structure of a smooth manifold of finite dimension in a natural manner.

Proof of Lemma 2.3.12: Fix $c>0$ with $\Omega_{\mu}^{c} \neq \emptyset$. We start by constructing a convenient partition on $[0,1]$.

Let $S \subset M$ be the ball $\{x \in M: \rho(p, x) \leq \sqrt{c}\}$. We know that all paths $\omega \in \Omega_{\mu}^{c}$ are contained in $S$, since for all $t \in I$

$$
\rho(p, \omega(t))^{2} \leq L(\omega)^{2} \leq E(\omega)<c
$$

Since $M$ is complete, we know $S$ is a compact set. Then there is an $0<\varepsilon<1$ such that, for all points $x, y \in S$, if $\rho(x, y)<\varepsilon$, then there is a unique geodesic from $x$ to $y$ of length $\varepsilon$, and such geodesic depend smoothly on the endpoints $x, y$.

We choose a partition $0=t_{0}<t_{1}<\cdots<t_{k}=1$ such that $t_{i+1}-t_{i}<\frac{\varepsilon^{2}}{c}$. 
For every element $\omega \in \Omega\left(t_{0}, \ldots, t_{k}\right)^{c}$, we know, by the Cauchy inequality, that

$$
L_{t_{i}}^{t_{i+1}}(\omega)=\left(t_{i=1}-t_{i}\right) E_{t_{i}}^{t_{i+1}}(\omega) \leq\left(t_{i+1}-t_{i}\right) E(\omega) \leq \varepsilon^{2}
$$

This implies that the restriction of $\omega$ to each interval $\left[t_{i}, t_{i+1}\right]$ is uniquely and smoothly determined by its endpoints.

Therefore, each $\omega$ is uniquely determined by the $(k-1)$-tuple

$$
\omega\left(t_{1}\right), \omega\left(t_{2}\right), \ldots, \omega\left(t_{k-1}\right) \in M \times \cdots \times M
$$

and this association defines an homeomorphism between $\operatorname{int} \Omega\left(t_{0}, \ldots, t_{k}\right)^{c}$ and an open set of the $(k-1)$-product $M \times \cdots \times M$. Using the differential structure which is induced by this product, we obtain the result.

To facilitate notation, we denote this manifold $\operatorname{int} \Omega\left(t_{0}, \ldots, t_{k}\right)^{c}$ by $B_{c}$ and $E^{\prime}: B_{c} \rightarrow \mathbb{R}$ is the restriction of the energy map to this space.

Theorem 2.3.13: For all $c>0$ with $\Omega_{\mu}^{c} \neq \emptyset$, the map $E^{\prime}: B_{c} \rightarrow \mathbb{R}$ is smooth. Also, for all $a<c$, the set $B_{c}^{a}=\left(E^{\prime}\right)^{-1}[0, a]$ is compact and is a deformation retract of $\Omega_{\mu}^{a}$. The critical points of $E^{\prime}$ are precisely the same of $E$ in $\Omega_{\mu}^{c}$ : the geodesics from $p$ to $q$ of length less then $\sqrt{c}$. The index (resp. the nullity) of the Hessian of $E_{* *}^{\prime}$ in each critical point $\gamma$ is equal to the index (resp. nullity) of $E_{* *}$ in $\gamma$.

Proof of Theorem 2.3.13: Since broken geodesics $\omega \in B_{c}$ depend smoothly on the $(k-1)$ tuple $\omega\left(t_{1}\right), \ldots, \omega\left(t_{k-1}\right) \in M \times \cdots \times M$, the map $E^{\prime}$ will also be smooth. We know from the Cauchy inequality that

$$
E^{\prime}(\omega)=\sum_{i=0}^{k-1} E_{t_{i}}^{t_{i+1}}(\omega)=\sum_{i=0}^{k-1} \frac{\rho\left(\omega\left(t_{i}\right), \omega\left(t_{i+1}\right)\right)}{t_{i+1}-t_{i}}
$$

Then we can see that, for each $a<c$, the space $B^{a}$ is homeomorphic to the space of the $(k-1)$ tuples $\left(y_{1}, \ldots, y_{k_{1}}\right) \in S \times \cdots \times S$, satisfying

$$
\sum_{i=0}^{k-1} \frac{\rho\left(y_{i}, y_{y+1}\right)}{t_{i+1}-t_{i}} \leq a
$$

Since this is a closed set on a compact space, $B^{a}$ is compact.

Using the same strategy we applied in the proof of Theorem 2.3.1, we construct a deformation retraction from $i n t \Omega_{\mu}^{c}$ to $B_{c}$ such that, for all $a<c$, its restriction is a deformation retraction from $\Omega_{\mu}^{a}$ to $B_{c}^{a}$. Each path $\omega \in \operatorname{int} \Omega_{\mu}^{c}$ will be taken to the only broken geodesic $r(\omega)$ of length $<\varepsilon$ such that $r(\omega)\left(t_{i}\right)=\omega\left(t_{i}\right)$. Since, for all $t \in I$

$$
\rho(p, \omega(t))^{2} \leq L(\omega)^{2} \leq E(\omega)<c
$$


we know that all such paths $\omega$ are contained in $S$ and therefore, this map $r$ is well-defined. Also,

$$
\begin{aligned}
E(r(\omega)) & =\sum_{i=0}^{k-1} E_{t_{i}}^{t_{i+1}}(r(\omega)) \\
& \leq \sum_{i=0}^{k-1} E_{t_{i}}^{t_{i+1}}(\omega) \\
& =E(\omega)<c
\end{aligned}
$$

We now construct a homotopy from the identity map to $r$ with the same strategy used in Theorem 2.3.1. That is, for all $u \in I$, we define $r_{u}: i n t \Omega_{\mu}^{c} \rightarrow i n t \Omega_{\mu}^{c}$ as follows. If $t_{i}<u \leq t_{i+1}$,

$$
\begin{aligned}
& \left.r_{u}(\omega)\right|_{\left[0, t_{i}\right]}=\left.r(\omega)\right|_{\left[0, t_{i}\right]} \\
& \left.r_{u}(\omega)\right|_{\left[t_{i}, u\right]}=\text { minimal geodesic from } \omega\left(t_{i}\right) \text { to } \omega(u) \\
& \left.r_{u}(\omega)\right|_{[u, 1]}=\left.\omega\right|_{[u, 1]}
\end{aligned}
$$

then $r_{0}=I d$ and $r_{1}=r$. Also, it is easily seen that $r_{u}(\omega)$ is continuous and that $r_{u}(\omega) \in$ int $\Omega_{\mu}^{c}$, for all $u$. This proves that $B_{c}$ is a deformation retract of $i n t \Omega_{\mu}^{c}$.

Moreover, since $E\left(r_{u}(\omega)\right) \leq E(\omega)$ for all $u$, we can use the same map to see that $B_{c}^{a}$ is a deformation retract of $\Omega_{\mu}^{a}$.

We move to the last part of the theorem which identifies the critical points, its indices and nullities.

First, we see that all geodesics are also broken geodesics and therefore all critical points of $E$ in int $\Omega_{\mu}^{c}$ are contained in $B_{c}$. Also, using the First Variation Formula, it is clear that the critical points $E^{\prime}$ are precisely the unbroken geodesics.

Finally, the tangent space $T_{\gamma} B_{c}$ of a geodesic $\gamma$ is naturally identified with the space of the broken Jacobi fields $T_{\gamma} \Omega\left(t_{0}, \ldots, t_{k}\right)$ defined above. This identification is due to the fact that, for any variation of $\gamma \bar{\alpha}:(-\delta, \delta) \rightarrow B_{c}$ through broken geodesics, its associated vector field will clearly be a Jacobi field along $\gamma$.

With these spaces identified, the statements regarding the index and nullity of the critical points are a direct consequence of Corollary 2.3.11.

An immediate consequence of the Theorem above and the theory developed in Section 2.1 is:

Theorem 2.3.14: If $M$ is a complete Riemmanian manifold and $p, q \in M$ are two points which are not conjugate along any geodesic of length $\leq \sqrt{a}$, then $\Omega_{\mu}^{a}$ has the homotopy type of a $C W$-complex, with one cell in dimension $\lambda$ for each geodesic in $\Omega_{\mu}^{a}$ at which $E_{* *}$ has index $\lambda$.

A more general approach was given by Bott in his paper [Bot59] and we close this section with some of its results.

Definition 2.3.8: We call the space $B_{c}$, constructed on Lemma 2.3.12, a model for $\Omega_{\mu}^{c}$. Let $\nu=(p, q ; h)$ be a triple consisting of two points $p, q \in M$ and a homotopy class $h$ of curves 
joining $p$ to $q$. We refer to such triples as base points on $M$. Given that $\nu$ and $\mu=(p, q)$, we define $\Omega_{\nu} M$ as the subset of all the elements of $\Omega_{\mu} M$ contained in $h$.

From the definition of homotopy groups, we know that $\pi_{n}\left(\Omega_{\mu}(M), \gamma\right)$ is the set of homotopy classes of maps $\left(S^{n}, s_{0}\right) \rightarrow\left(\Omega_{\mu}(M), \gamma\right)$. Since all $S^{n}$ 's are connected, we know that all such maps are contained in the connected component of $\gamma$ in $\Omega_{\mu}(M)$, which is precisely the set of all paths which are homotopic to $\gamma$. Therefore we see that studying homotopy groups of $\Omega_{\nu} M$ is the same as studying $\Omega_{\mu} M$ with basepoint $h=[\gamma]$.

Definition 2.3.9: $\quad$ Let $\nu$ be a base point on $M$. The space $\Omega_{\nu} M$ is called non-degenerate if the set of geodesics in $\Omega_{\nu} M$ is the union of non-degenerate critical manifolds. Precisely: $\Omega_{\nu} M$ is non-degenerate if, given any regular half-space $\Omega_{\nu}^{a} M$ with model $\phi_{n}^{b}\left(M^{n}\right)$, the critical set of $\phi_{n}$ on $\phi_{n}^{b}\left(M^{n}\right)$ is the disjoint union of non-degenerate critical manifolds.

Theorem 2.3.15: (Suspension Theorem) Let $\Omega_{\nu} M$ be non-degenerate. Let $\mathcal{C}$ be the collection of critical manifolds in $\Omega_{\nu} M$. Let it be well-ordered, $\mathcal{C}=\left\{C_{1}, C_{2}, \ldots\right\}$, compatible with the partial order defined on $\mathcal{C}$ by the length of geodesics, and let $\eta_{i}^{-}$be the negative bundle of $C_{i}$. Then $\Omega_{\nu} M$ has the same homotopy groups as the $C W$-complex

$$
K=\eta_{1}^{-} \cup \eta_{2}^{-} \cup \eta_{3}^{-} \cup \ldots
$$

The result above follows from the same "direct-limit" argument used in the proof of Theorem 2.3.1, combined with Theorem 2.3.13 and the results of Section 2.2.

The statement above can be easily sharpened by the Fundamental Theorem of Morse Theory:

Theorem 2.3.16: $\quad$ Let $M$ be a complete Riemannian manifold, and let $p, q \in M$ be two points which are not conjugate along any geodesic. Then $\Omega_{\mu}(M)$ has the homotopy type of a countable $C W$-complex which contains one cell of dimension $\lambda$ for each geodesic from $p$ to $q$ of index $\lambda$.

Corollary 2.3.17: $\quad \Omega_{\mu}(M)$ has the homotopy type of a $C W$-complex.

Example 2.3.1: $\quad$ We apply the above procedure to spheres $S^{n}$. Two points $p, q \in S^{n}$ are conjugate if and only if $q$ is the antipodal point of $p$ and that its multiplicity as conjugate points is $n-1$.

Suppose that $p$ and $q$ are two non-conjugate points on the sphere $S^{n}$, i.e. if $p^{\prime}$ and $q^{\prime}$ are the antipodal points of $p$ and $q$, respectively, then $p \neq q^{\prime}$. Then we know there are enumerably many geodesics $\gamma_{0}, \gamma_{1}, \gamma_{2}, \ldots$ from $p$ to $q$. Precisely, let $\gamma_{0}$ be the short great circle arc from $p$ to $q$; let $\gamma_{1}$ denote the long great circle arc, which we represent as $p q^{\prime} p^{\prime} q$; let $\gamma_{2}$ denote the arc $p q p^{\prime} q^{\prime} p q$; and so on (Figure 2.7). Then we see that the index $k$ denotes precisely the amount of times the geodesic $\gamma_{k}$ passes the point $p^{\prime}$.

From Morse' Index Theorem, we know that the index of $\gamma_{k}$ is given by

$$
\lambda\left(\gamma_{k}\right)=k(n-1)
$$



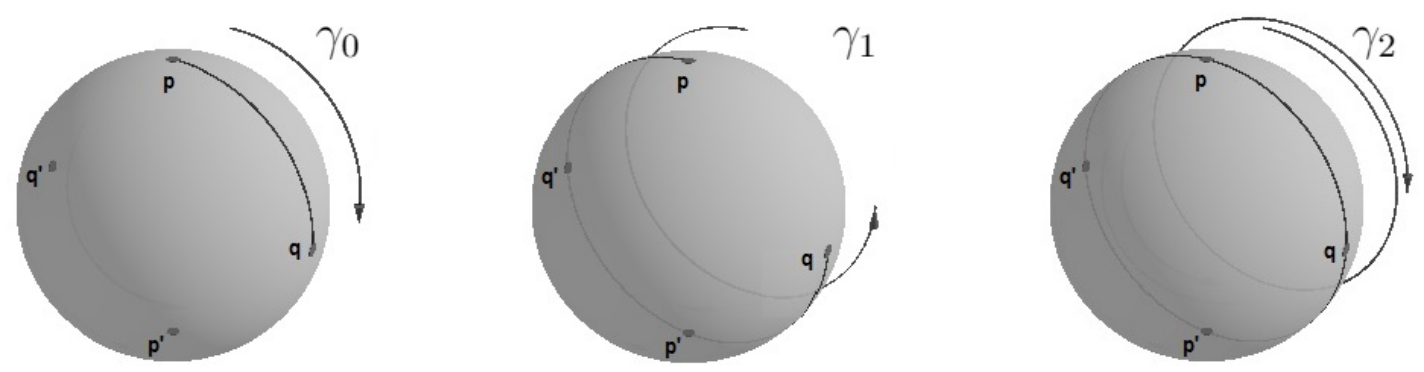

Figure 2.7: Curves $\gamma$ on the sphere

Therefore, we have:

Corollary 2.3.18: $\quad$ The loop space $\Omega S^{n}$ has the homotopy type of a $C W$-complex with one cell in dimension $k(n-1)$, for each positive integer $k$. In particular, this means that $\Omega S^{n}$ is homotopy equivalent to $S^{n-1} \cup e^{2(n-1)} \cup e^{3(n-1)} \cup \ldots$, and as a consequence

$$
\pi_{k}\left(S^{n-1}\right)=\pi_{k+1}\left(S^{n}\right)
$$

The above procedure can be generalised:

Definition 2.3.10: $\quad$ Let $M^{\nu}$ be the set of all geodesics of minimal length which join $p$ to $q$ and are contained in $h$.

We define a very natural map of the suspension of $M^{\nu}$ into $M$, which assigns to a pair $(\gamma, t)$, $\gamma \in M^{\nu}$ and $t \in[0,1]$, the point $\gamma(t)$ (for a fixed small $t>0$, this map is 1-to- 1 on $M^{\nu}$ and serves to define a topology on $\left.M^{\nu}\right)$. The induced homomorphism of $\pi_{k}\left(M^{\nu}\right)$ to $\pi_{k+1}(M)$ will be denoted by $\nu_{*}$.

Also, we write $|\nu|$ for the first strictly positive integer which occurs as the index of some geodesic in $\Omega_{\nu} M$

Corollary 2.3.19: $\quad$ Let $\Omega_{\nu} M$ be non-degenerate, then

$$
\nu_{*}: \pi_{r}\left(M^{\nu}\right) \rightarrow \pi_{r+1}(M) \quad 0<r<|\nu|-1
$$

is an isomorphism onto.

This section will finish with a comment on the implications of the results above and how it was later related to the other theories developed in this chapter.

Although Morse-Bott theory yields very interesting results and was strongly used on Bott's proof of the Periodicity Theorems, it is actually not needed when studying the loop space of a manifold. In fact, in his book [Mil63] of 1963, Milnor shows that the classical Morse Theory, combined with the results developed above, provide enough tools to prove the Periodicity Theorems. The main reason is that, if $M$ is a complete manifold and $p \in M$, then almost all points $q \in M$ are non-conjugate to $p$.

Theorem 2.3.20: $\quad$ Let $M$ be a complete manifold. Then a point $\exp _{p} v$ is a conjugate point to $p$ 
along the geodesic $\gamma_{v}(t)=\exp _{p}(t v)$ if and only if $v$ is a critical point of the map $\exp _{p}: T_{p} M \rightarrow M$.

Proof of Theorem 2.3.20: Let $v$ be a critical point of $\exp _{p}$ in $T_{p} M$. Then there is a non-zero $X \in T_{v}\left(T_{p} M\right)$, such that $\exp _{*}(X)=0$. Let $u \rightarrow v(u)$ be a map in $T_{p} M$ such that $v(0)=0$ and $\frac{d v}{d u}(0)=X$. We construct a map $\alpha$, defined by $\alpha(u, t)=\exp _{p} t v(u)$, which is a variation through geodesics of $\gamma_{v}$. This gives us a Jacobi field $W$ along $\gamma_{v}$, which is given by $W(t)=\left.\frac{\partial\left(\exp _{p} t v(u)\right)}{\partial u}\right|_{u=0}$. Since $\alpha(u, 0)=p$, for all $u$, we know $W(0)=0$. Also

$$
W(1)=\left.\frac{\partial\left(\exp _{p} v(u)\right)}{\partial u}\right|_{u=0}=\exp _{*} \frac{d v(u)}{d u}(0)=\exp _{*} X=0
$$

Hence, $W$ is a Jacobi field along $\gamma_{v}$ which vanishes at both $p$ and $q$ and is not identically zero because

$$
\frac{D W}{d t}(0)=\left.\frac{D}{\partial u} \frac{\partial\left(\exp _{p} t v(u)\right)}{\partial t}\right|_{(0,0)}=\left.\frac{d v(u)}{\partial u}\right|_{u=0}=X \neq 0
$$

Therefore, $p$ and $\exp _{p} v$ are conjugate points along $\gamma_{v}$.

On the other hand, if $v$ is not a critical point of $\exp _{p}$, we know there are $X_{1}, \ldots, X_{n}$ linearly independent in $T_{v}\left(T_{p} M\right)$, such that $\exp _{*} X_{1}, \ldots, \exp _{*} X_{n}$ are linearly independent in $T_{p} M$. Repeating the procedure above, we obtain paths $v_{1}, \ldots, v_{n}$ in $T_{p} M$ with $v_{i}(0)=0$ and $\frac{d v_{i}}{d u}=X_{i}$, and, through these paths, we construct variations of $\gamma_{v}$ through geodesics $\alpha_{i}(u, t)=\exp _{p} t v_{i}(u)$. Again, we get Jacobi fields along $\gamma_{v}$, given by $W_{i}(t)=\left.\frac{\partial\left(\exp _{p} t v_{i}(u)\right)}{\partial u}\right|_{u=0}$. However, now we have

$$
W_{i}(1)=\left.\frac{\partial\left(\exp _{p} v_{i}(u)\right)}{\partial u}\right|_{u=0}=\exp _{*} \frac{d v_{i}(u)}{\partial u}(0)=\exp _{*} X_{i} \neq 0
$$

Even more, since $\exp _{*} X_{1}, \ldots, \exp _{*} X_{n}$ are linearly independent in $T_{p} M$, we see that no non-trivial combination of the Jacobi fields $W_{i}$ will vanish at $\exp _{p} v$. Since $n$ is precisely the dimension of the space of Jacobi fields along $\gamma_{v}$ which vanish at $p$, we conclude there is no non-trivial Jacobi field along $\gamma_{v}$, which vanishes at both $p$ and $\exp _{p} v$.

With the theorem above and Sard's Theorem, we get:

Corollary 2.3.21: Let $p \in M$. For almost all $q \in M, p$ is not conjugate to $q$ along any geodesic.

This result assures us that we can always choose $\mu$ to be a pair of non-conjugate points. This makes $\Omega_{\mu} M$ satisfy the hypothesis of Theorem 2.3.16 and we see that, by making this choice, we can apply the Classical Morse Theory.

Now that we have constructed the extension of the previous theories to the loop space and derived results on its cellular structure, we will apply them to derive Bott's Periodicity Theorems. 


\subsection{The Periodicity Theorem for the Classical Groups}

Equipped with the results above, Bott applied them to yield relations between the homotopy groups of manifolds $M$ and the space of it's minimal geodesics. It then made sense to study manifolds in which geodesics had a nice description, for instance, symmetric spaces. These special kind of manifolds were already studied by Bott and he had produced many results regarding its geodesics (see [BS58]). Amongst the famous symmetric spaces, we have all compact Lie Groups and, in particular, the Classical Groups.

Definition 2.4.1: A symmetric space is a connected Riemmanian manifold $M$ such that, for each $p \in M$, there is an isometry $I_{p}: M \rightarrow M$ which leaves $p$ fixed and reverses geodesics through $p$, i.e., if $\gamma$ is a geodesic and $\gamma(0)=p$ then $I_{p}(\gamma(t))=\gamma(-t)$.

One of the main results on Bott's article [Bot59] is:

Proposition 2.4.1: If $M$ is a symmetric space, then $\Omega_{\nu} M$ is non-degenerate for every base point $\nu=(p, q, h)$ in $M$.

The result above guarantees that, for all symmetric spaces $M$, the space $\Omega_{\nu} M$ is on the hypothesis of Corollary 2.3.19 and yields:

Theorem 2.4.2: $\quad$ For every symmetric space $M$,

$$
\pi_{k}\left(M^{\nu}\right)=\pi_{k+1}(M) \quad 0<k<|\nu|-1
$$

Although this is quite useful, Bott showed even more:

Proposition 2.4.3: $\quad$ Let $M$ be a symmetric space and $\nu=(p, q, h)$ a base point in $M$. If $M^{\nu}$ is the set of all geodesics of minimal length joining $p$ to $q$ contained in $h$, then $M^{\nu}$ is again a symmetric space.

This means that we could iterate the process of passing from $M$ to $M^{\nu}$. To facilitate notation, we call a sequence of symmetric spaces ... $M_{1} \rightarrow M_{2} \rightarrow M_{3} \ldots$ a $\nu$-sequence if, at each step, $M_{i}=M_{i+1}^{\nu}$ for some appropriate base point $\nu$ in $M_{i+1}$. For example, since $S^{n-1}$ is identified to the space of minimal geodesics between antipodal points in $S^{n}$, we see that $\ldots S^{n-1} \rightarrow S^{n} \rightarrow$ $S^{n+1} \ldots$ is a $\nu$-sequence.

The main result in Bott's paper regarding the Classical Groups is:

Theorem 2.4.4: $\quad$ The following are three $\nu$-sequences with the value of $|\nu|$ indicated above the arrows at each step:

$$
\begin{aligned}
& \frac{U(2 n)}{U(n) \times U(n)} \stackrel{2 n+2}{\longrightarrow} U(2 n) \\
& \frac{O(2 n)}{O(n) \times O(n)} \stackrel{n+1}{\longrightarrow} \frac{U(2 n)}{O(2 n)} \stackrel{2 n+1}{\longrightarrow} \frac{S p(2 n)}{U(2 n)} \stackrel{4 n+2}{\longrightarrow} S p(2 n) \\
& \frac{S p(2 n)}{S p(n) \times S p(n)} \stackrel{4 n+1}{\longrightarrow} \frac{U(4 n)}{S p(2 n)} \stackrel{8 n-2}{\longrightarrow} \frac{S O(8 n)}{U(4 n)} \stackrel{8 n-2}{\longrightarrow} S O(8 n)
\end{aligned}
$$


Bott proved that those were $\nu$-sequences by choosing nice base points and exhibiting the spaces of minimal geodesics, based on results about symmetric spaces and the classical groups, which are not in the scope of this dissertation. However, we show how the result above immediately leads to the periodicity theorems:

Lemma 2.4.5: For all the spaces in Theorem 2.4.4, the homotopy groups $\pi_{k}$ become independent of $n$ if $n \gg k$. Also, we have the following equivalences between stable homotopy groups:

$$
\begin{aligned}
\lim _{n \rightarrow \infty} \pi_{k+1}\left(\frac{U(2 n)}{U(n) \times U(n)}\right) & =\pi_{k}(U) \\
\lim _{n \rightarrow \infty} \pi_{k+1}\left(\frac{O(2 n)}{O(n) \times O(n)}\right) & =\pi_{k}(O) \\
\lim _{n \rightarrow \infty} \pi_{k+1}\left(\frac{S p(2 n)}{S p(n) \times S p(n)}\right) & =\pi_{k}(S p)
\end{aligned}
$$

Proof of Lemma 2.4.5: We show the stability of the homotopy groups of $\frac{U(2 n)}{U(n) \times U(n)}$ and equivalence 2.11. The result for the other spaces follow analogously.

We know there is a fibration $U(n) \hookrightarrow U(2 n) \rightarrow \frac{U(2 n)}{U(n)}$, which gives us a long exact sequence of homotopy groups

$$
\cdots \longrightarrow \pi_{k}(U(n)) \longrightarrow \pi_{k}(U(2 n)) \longrightarrow \pi_{k}\left(\frac{U(2 n)}{U(n)}\right) \longrightarrow \pi_{k-1}(U(n)) \longrightarrow \pi_{k-1}(U(2 n)) \longrightarrow \cdots
$$

If $n \gg k$, then the maps $\pi_{k}(U(n)) \rightarrow \pi_{k}(U(2 n))$ will be isomorphisms, and therefore, $\pi_{k}\left(\frac{U(2 n)}{U(n)}\right)=$ 0 .

Applying the same argument to the fibration $U(n) \hookrightarrow \frac{U(2 n)}{U(n)} \rightarrow \frac{U(2 n)}{U(n) \times U(n)}$, we get the stability of the homotopy groups and equivalence 2.11 .

Combining the result above with Corollary 2.3 .19 and the fact that the values $|\nu|$ tend to $\infty$ with $n$ at each step of the sequences in Theorem 2.4.4, we finally get:

\section{Theorem 2.4.6: (The Periodicity Theorem for the Classical Groups)}

The stable homotopy of the classical groups is periodic:

$$
\begin{aligned}
\pi_{k}(U) & =\pi_{k+2}(U) \\
\pi_{k}(O) & =\pi_{k+4}(S p) \\
\pi_{k}(S p) & =\pi_{k+4}(O)
\end{aligned}
$$

We will finish this section defining two spaces which are central to K-Theory and whose nice properties are inherited from the periodicity theorem:

Definition 2.4.2: $\quad$ Let BO be the space defined as the direct limit of the sequence of spaces

$$
\cdots \rightarrow \frac{O(2 n)}{O(n) \times O(n)} \rightarrow \frac{O(2(n+1))}{O(n+1) \times O(n+1)} \rightarrow \ldots
$$


If we think of this space through the telescoping construction, we see that any map $S^{k} \rightarrow B O$ is homotopic to a map $S^{k} \rightarrow \frac{O(2 n)}{O(n) \times O(n)}$ for some $n \gg k$. By Lemma 2.4.5. we see that $\pi_{k+1}(B O) \simeq \pi_{k}(O)$ and therefore

$$
\begin{array}{c|cccccccc}
i(\bmod 8) & 0 & 1 & 2 & 3 & 4 & 5 & 6 & 7 \\
\hline \pi_{i}(B O) & \mathbb{Z} & \mathbb{Z}_{2} & \mathbb{Z}_{2} & 0 & \mathbb{Z} & 0 & 0 & 0
\end{array}
$$

Keeping track of the components, the maps implicit in 2.9 and 2.10 can be interpreted as maps

$$
\begin{gathered}
\lambda_{\mathbb{R}}^{\prime}: B O \rightarrow \Omega^{8}(B O) \\
\lambda_{\mathbb{R}}: B O \times \mathbb{Z} \rightarrow \Omega^{8}(B O \times \mathbb{Z})
\end{gathered}
$$

which induces isomorphisms in all homotopy groups and therefore, by Whitehead's Theorem, a homotopy equivalence.

An analogous construction can be done to the unitary group:

Definition 2.4.3: $\quad$ Let $B U$ be the space defined as the direct limit of the sequence of spaces

$$
\cdots \rightarrow \frac{U(2 n)}{U(n) \times U(n)} \rightarrow \frac{U(2(n+1))}{U(n+1) \times U(n+1)} \rightarrow \cdots
$$

From the arguments used for $B O$, we see that $\pi_{k+1}(B U)=\pi_{k}(U)$, and therefore $\pi_{2 n}(B U)=\mathbb{Z}$ and $\pi_{2 n+1}(B U)=0$. Also, we construct a homotopy equivalence

$$
\begin{gathered}
\lambda_{\mathbb{C}}^{\prime}: B U \rightarrow \Omega^{2}(B U) \\
\lambda_{\mathbb{C}}: B U \times \mathbb{Z} \rightarrow \Omega^{2}(B U \times \mathbb{Z})
\end{gathered}
$$

Therefore, using the Periodicity Theorem, we have exhibited spaces $B O$ and $B U$ with periodic homotopy groups. 


\section{Chapter 3}

\section{An Introduction to K-Theory}

In this chapter we give a brief introduction to Topological K-Theory focusing on how it relates to Bott's Periodicity Theorem.

We start by giving an introductory approach to representable functors and generalised cohomology theories in Section 3.1. highlighting how the Periodicity Theorem naturally leads one to the production of the functors $K O$ and $K$. The main references for this section are [Bot70], [Swi75] and [May99].

In section 3.2 , we give a brief presentation to the ideas behind the geometrical interpretation of K-Theory. We highlight its relation to the spaces $B O$ and $B U$ and emphasise how the geometrical and representable approaches combine to yield many important aspects of this theory. The main references to this section are [Bot70], [AA67], [Bot69], [Swi75] and [Hat03].

\subsection{Cohomology Theories}

Historically, cohomology theory was first defined on simplicial complexes and was constructed using methods which would make it explicitly computable from the combinatorial structure of the complex. From such initial definitions, the question of whether such theory was homotopy invariant was a difficult matter which took quite some time to be solved.

The search for proofs of the homotopy invariance of $H_{\text {simp }}^{n}(X, \pi)$ led to the definition of many cohomology theories such as singular, cellular and Cech cohomologies. Each of them started from a different geometrical point of view and produced a theory with different properties on different categories of spaces.

One very interesting approach on the creations of cohomology theories, which turned out to be incredibly efficient in CW-complexes, is to construct it by using contravariant functors which we already know respect homotopy equivalences. One such functor $F$, which is defined on the category of pointed topological spaces, can be easily described: fix a pointed space $\left(K, k_{0}\right)$ and 
set, for all $\left(X, x_{0}\right)$,

$$
F\left(X, x_{0}\right)=\left[\left(X, x_{0}\right) ;\left(K, k_{0}\right)\right]
$$

the based homotopy classes of maps from $\left(X, x_{0}\right)$ to $\left(K, k_{0}\right)$. Also, given a map $g:\left(X, x_{0}\right) \rightarrow$ $\left(Y, y_{0}\right)$ we define $g^{*}: F\left(Y, y_{0}\right) \rightarrow F\left(X, x_{0}\right)$, by $g^{*}[h]=[h \circ g]$. It is simple to check that this is indeed a functor since the identities $I d^{*}=I d$ and $(g \circ h)^{*}=g^{*} \circ h^{*}$ follow immediately from the definition.

Analogously, one can construct covariant functors $G$ by fixing a pointed space $\left(K, k_{0}\right)$ and defining

$$
G\left(X, x_{0}\right)=\left[\left(K, k_{0}\right) ;\left(X, x_{0}\right)\right]
$$

and, from a map $g: X \rightarrow X^{\prime}$, setting $g_{*}: G(X) \rightarrow G\left(X^{\prime}\right)$ by $g_{*}[h]=[g \circ h]$.

It is simple to see that such functors $F$ and $G$ are clearly homotopy invariant, since, from the definition $f \sim g$ implies $f^{*}=g^{*}$.

Functors of this type are called representable, and we call the space $\left(K, k_{0}\right)$ a classifying space for $F$, or equivalently, that $F$ is represented by $\left(K, k_{0}\right)$.

Since we wish to create a cohomology theory, it is natural to ask under what circumstances a representable functor takes its values in groups. This leads us to:

Definition 3.1.1: An $H$-group is a pointed space $\left(K, k_{0}\right)$ with a multiplication map $\mu: K \times$ $K \rightarrow K$ satisfying the following properties:

- $k_{0}$ is a homotopy identity: there exists a homotopy between the maps $\mu \circ\left(I d, k_{0}\right), \mu \circ\left(k_{0}, I d\right)$ and Id, i.e. if the following diagram commutes:

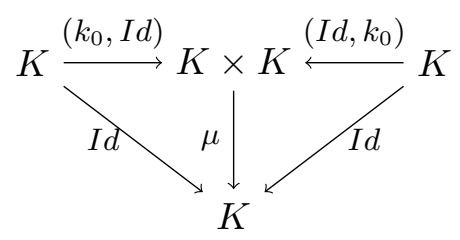

- $\mu$ is homotopy associative: there exists a homotopy between the maps $\mu \circ(\mu \times I d)$ and $\mu(I d \times \mu)$, i.e. if the following diagram commutes:

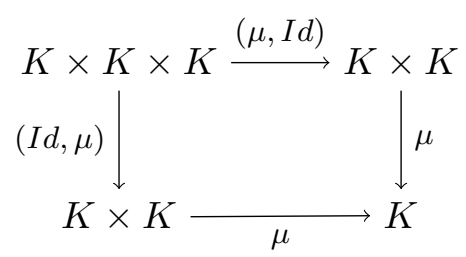

- All elements in $K$ have a homotopy inverse $\nu$ : there is a map $\nu:\left(K, k_{0}\right) \rightarrow\left(K, k_{0}\right)$ such that the maps $\mu \circ(I d \times \nu)$ and $\mu \circ(\nu \times I d)$ are both homotopic to the constant map $k_{0}$, i.e. if the following diagram commutes: 


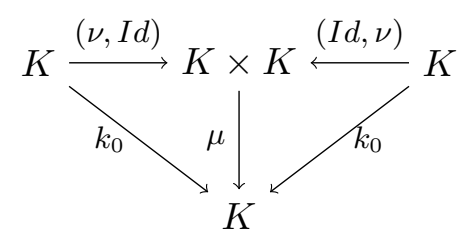

Also, an H-group is called abelian if $\mu$ is homotopy commutative. This means that, if $T$ : $K \times K \rightarrow K \times K$ takes $\left(k_{1}, k_{2}\right) \rightarrow\left(k_{2}, k_{1}\right)$, there is a homotopy between $\mu$ and $\mu \circ T$, i.e, the following diagram commutes:

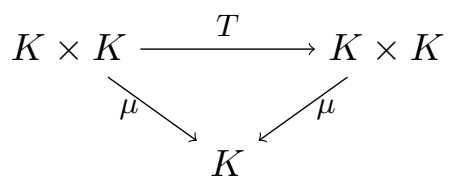

To facilitate notation, we omitted the base points. However, we expect all homotopies to be relative to base points.

So we think of an $H$-group as a group where all the equivalences are "up-to-homotopy".

Example 3.1.1: $\quad$ Every topological group is evidently an $H$-group .

Example 3.1.2: $\quad$ The loopspace $\Omega\left(X, x_{0}\right)$ is an $H$-group when endowed with the operation of concatenating curves. Also, iterations $\Omega^{n}\left(X, x_{0}\right)$ are $H$-groups and are known to be abelian.

Proposition 3.1.1: If $\left(K, k_{0}\right)$ is an $H$-group with multiplication $\mu$ and homotopy inverse $\nu$, then, for every $\left(X, x_{0}\right)$, the set

$$
\left[\left(X, x_{0}\right) ;\left(K, k_{0}\right)\right]
$$

can be given a structure of a group if we define the product $[f] \cdot[g]$ to be the homotopy class of the composition

$$
X \stackrel{\Delta}{\rightarrow} X \times X \stackrel{f \times g}{\rightarrow} K \times K \stackrel{\mu}{\rightarrow} K
$$

where $\Delta$ is the diagonal map given by $\Delta(x)=(x, x)$. The identity of the group is the class of the constant map $k_{0}$ and the inverse is given by $[f]^{-1}=[\nu \circ f]$. Also, if $\mu$ is homotopy commutative, then $\left[\left(X, x_{0}\right) ;\left(K, k_{0}\right)\right]$ is an abelian group. Every map $f:\left(X, x_{0}\right) \rightarrow\left(Y, y_{0}\right)$ induces a homomorphism $f^{*}:\left[\left(Y, y_{0}\right) ;\left(K, k_{0}\right)\right] \rightarrow\left[\left(X, x_{0}\right) ;\left(K, k_{0}\right)\right]$.

This result implies that good candidates to be used as classifying spaces when trying to produce a cohomology theory would be loop spaces. The proof of the result is straightforward and is seen in details in [Swi75], Proposition 2.14.

We also look at the definition of what would be the dual space of an $H$-group, which will play an important role hereon:

Definition 3.1.2: $\quad$ An $H$-cogroup is a pointed space $\left(K, k_{0}\right)$ with a continuous multiplication map $\mu^{\prime}: K \rightarrow K \vee K$ satisfying the following properties:

- $k_{0}$ is a homotopy identity: there exists a homotopy between the maps $\left(I d, k_{0}\right) \circ \mu^{\prime},\left(k_{0}, I d\right) \circ \mu^{\prime}$ 
and Id (where $\left(k_{0}, I d\right)$ is the map such that $\left(k_{0}, I d\right)\left(k, k_{0}\right)=k_{0}$ and $\left(k_{0}, I d\right)\left(k_{0}, k\right)=k$, for all $k \in K)$, i.e. if the following diagram commutes:

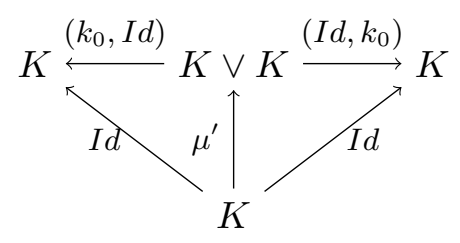

- $\mu$ is homotopy associative: there exists a homotopy between the maps $\left(\mu^{\prime} \vee I d\right) \circ \mu^{\prime}$ and $\left(I d \vee \mu^{\prime}\right) \circ \mu^{\prime}$, i.e. if the following diagram commutes:

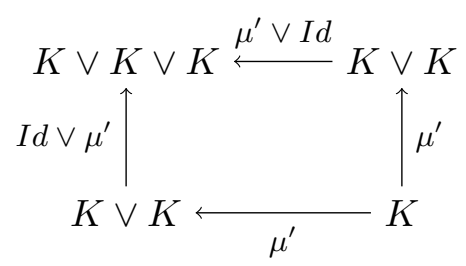

- All elements in $K$ have a homotopy inverse $\nu^{\prime}$ : there is a map $\nu^{\prime}:\left(K, k_{0}\right) \rightarrow\left(K, k_{0}\right)$ such that the maps $\left(I d \times \nu^{\prime}\right) \circ \mu^{\prime}$ and $\left(\nu^{\prime} \times I d\right) \circ \mu^{\prime}$ are both homotopic to the constant map $k_{0}$, i.e. if the following diagram commutes:

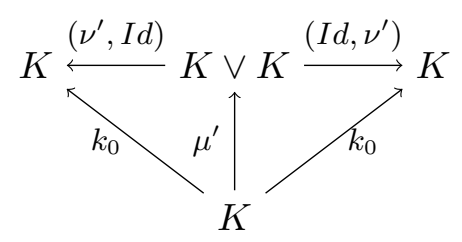

Also, an H-group is called abelian if $\mu^{\prime}$ is homotopy commutative. This means that, if $T$ : $K \vee K \rightarrow K \vee K$ takes $\left(k_{1}, k_{2}\right) \rightarrow\left(k_{2}, k_{1}\right)$, there is a homotopy between $\mu^{\prime}$ and $T \circ \mu^{\prime}$, i.e, the following diagram commutes:

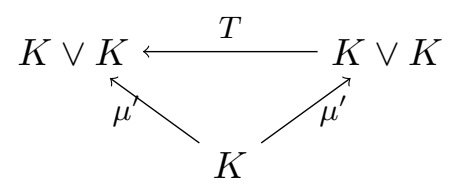

To facilitate notation, we omitted the base points. However, we expect all homotopies to be relative to base points.

Proposition 3.1.2: $\quad$ If $\left(K, k_{0}\right)$ is an $H$-cogroup with multiplication $\mu^{\prime}$ and homotopy inverse $\nu^{\prime}$, then, for every $\left(X, x_{0}\right)$, the set

$$
\left[\left(K, k_{0}\right) ;\left(X, x_{0}\right)\right]
$$

can be given a structure of a group if we define the product $[f] \cdot[g]$ to be the homotopy class of the composition

$$
K \stackrel{\mu^{\prime}}{\rightarrow} K \vee K \stackrel{f \vee g}{\longrightarrow} X \vee X \stackrel{\Delta^{\prime}}{\longrightarrow} X
$$

where $\Delta^{\prime}$ is the folding map given by $\Delta^{\prime}\left(x, x_{0}\right)=x=\Delta^{\prime}\left(x_{0}, x\right)$. The identity of the group will 
be the class of the constant map $x_{0}$ and the inverse will be given by $[f]^{-1}=\left[f \circ \nu^{\prime}\right]$. Also, if $\mu^{\prime}$ is homotopy commutative, then $\left[\left(K, k_{0}\right) ;\left(X, x_{0}\right)\right]$ is an abelian group. Every map $f:\left(X, x_{0}\right) \rightarrow$ $\left(Y, y_{0}\right)$ induces a homomorphism $f^{*}:\left[\left(K, k_{0}\right) ;\left(X, x_{0}\right)\right] \rightarrow\left[\left(K, k_{0}\right) ;\left(Y, y_{0}\right)\right]$

Again, the proof of the result above is straightforward and is seen in details in Swi75, Proposition 2.21 .

We now construct one of the most important examples of $H$-cogroups. We start by recalling the definitions of smash product and reduced suspension, which are thought of as somehow analogues on the category of pointed spaces to the product and the usual suspension:

Definition 3.1.3: Given two pointed spaces $\left(X, x_{0}\right),\left(Y, y_{0}\right)$, we define the smash product $(X \wedge Y, *)$ to be the space

$$
X \wedge Y=\frac{X \times Y}{X \vee Y}
$$

with base point $*=p(X \vee Y)$, where $p: X \times Y \rightarrow X \wedge Y$ is the projection.

Given a pointed space $\left(X, x_{0}\right)$, we define the reduced suspension $(S X, *)$ (which will sometimes be called simply by suspension when it is clear from the context) to be the smash product $\left(S^{1} \wedge X, *\right)$.

Example 3.1.3: $\quad$ The suspension $(S X, *)$ can be endowed with an $H$-cogroup structure.

This structure is constructed by defining $\mu^{\prime}:(S X, *) \rightarrow(S X \vee S X, *)$ to be the map obtained by collapsing the "equator" of $S X$ into a point. The description of this map and the proof that it can be used to produce an $H$-cogroup structure in $S X$ is seen in Example 2.22 of [Swi75].

Lemma 3.1.3: Let $\left(X, x_{0}\right),\left(Y, y_{0}\right)$ and $\left(Z, z_{0}\right)$ be Hausdorff and locally compact, then

- $X \wedge(Y \wedge Z) \simeq(X \wedge Y) \wedge Z$;

- $S^{1} \wedge S^{n} \simeq S^{n+1}$;

- There is a natural equivalence

$$
A:\left[(X \wedge Y, *) ;\left(Z, z_{0}\right)\right] \rightarrow\left[\left(Y, y_{0}\right) ;\left(\left(Z, z_{0}\right)^{\left(X, x_{0}\right)}, f_{0}\right)\right]
$$

defined by $A[f]=[\hat{f}]$ where $\hat{f}: Y \rightarrow Z^{X}$ is given by $(\hat{f}(y))(x)=f[x, y]$.

Proof of Lemma 3.1.3: We now prove the third property described above. We start by showing that $A$ is surjective. Indeed, given a map $g:\left(Y, y_{0}\right) \rightarrow\left(\left(Z, z_{0}\right)^{\left(X, x_{0}\right)}\right)$, we define a continuous $f^{\prime}:\left(X \times Y,\left(x_{0}, y_{0}\right)\right) \rightarrow\left(Z, z_{0}\right)$ by setting $f^{\prime}(x, y)=(g(y))(x)$. We see that $f^{\prime}(X \vee Y)=z_{0}$ and therefore, passing to the quotient, we get a continuous map $f=p \circ f^{\prime}:(X \wedge Y, *) \rightarrow\left(Z, z_{0}\right)$ given by $f([x, y])=f^{\prime}(x, y)=(g(y))(x)$. Then $A[f]=[g]$.

Regarding injectivity, if $f, g:(X \wedge Y, *) \rightarrow\left(Z, z_{0}\right)$ are maps which have the same image under $A$, then there exists a homotopy $F: I \times Y \rightarrow Z^{X}$ relative to $x_{0}$, such that $F_{0}=\hat{f}$ and $H_{1}=\hat{g}$. From this we define $H^{\prime}: I \times(X \times Y) \rightarrow Z$ given by $H^{\prime}(t,(x, y))=F(t, y)(x)$, which we know to be a continuous homotopy relative to $\left(x_{0}, y_{0}\right)$. Also, for all $t \in I$, we have $H^{\prime}(t, X \vee Y)=z_{0}$ 
and therefore, passing to the quotient, we get a continuous map $H: I \times(X \wedge Y) \rightarrow Z$ given by $H(t,[x, y])=H^{\prime}(t,(x, y))=F(t, y)(x)$ which is a homotopy relative to the basepoint of $X \wedge Y$. Also $H_{0}[x, y]=H_{0}^{\prime}(x, y)=F(0, y)(x)=\hat{f}(x)=f[x, y]$, and similarly, we see that $H_{1}[x, y]=g$. Hence $f \simeq g$.

An important application of the results above which is used later is:

Corollary 3.1.4: For every Hausdorff space $X$, there is a natural equivalence:

$$
A:\left[(S X, *) ;\left(Y, y_{0}\right)\right] \rightarrow\left[\left(X, x_{0}\right) ;\left(\Omega(Y), f_{0}\right)\right]
$$

It is natural to enquire whether studying representable functors gives a certain generality on the scope of all the contravariant functors which could be used to construct a cohomology theory. This is the case: Brown's Representability Theorem assures us that any contravariant functors defined on the category of based $\mathrm{CW}$-complexes, $\mathscr{P} \mathscr{W}$, satisfying reasonable properties is representable, even more, it states that it can be represented by a CW-complex.

We prove such a theorem by assuming some results on CW-complexes which are found in Chapter 6 of Swi75.

We know that for any CW-complex $\left(Y, y_{0}\right)$, and any element $u \in F\left(Y, y_{0}\right)$, we have a natural transformation

$$
\begin{aligned}
T_{u}:\left[\left(X, x_{0}\right) ;\left(Y, y_{0}\right)\right] & \rightarrow F\left(X, x_{0}\right) \\
f & \rightarrow f^{*}(u)
\end{aligned}
$$

What we show on the proof of Brown's Representability Theorem is that, for a wide class of such functors $F$, we can find a CW-complex $\left(Y, y_{0}\right)$ and an element $u \in F\left(Y, y_{0}\right)$ for which the map above is an equivalence.

Since we are working on pointed spaces, it is convenient to use, instead of $I \times\left(X, x_{0}\right)$, the homotopy equivalent space $I^{+} \wedge X=\frac{I \times X}{I \times\left\{x_{0}\right\}}$, with basepoint [0, $\left.x_{0}\right]$. Also, to facilitate notation, if $i: A \rightarrow X$ is an inclusion and $u \in F\left(X, x_{0}\right)$, we will denote $i^{*}(u) \in F\left(A, x_{0}\right)$ by $\left.u\right|_{A}$.

Precisely, we consider contravariant functors $F$ on $\mathscr{P} \mathscr{W}$, the category of pointed $\mathrm{CW}$-complexes, satisfying the following two properties:

Wedge axiom: given a wedge sum $\bigvee_{\alpha} X_{\alpha}$, the inclusions $i_{\beta}: X_{\beta} \rightarrow \bigvee_{\alpha} X_{\alpha}$ induce an isomorphism

$$
\left\{i_{\alpha}^{*}\right\}: F\left(\vee_{\alpha} X_{\alpha}\right) \rightarrow \prod_{\alpha} F\left(X_{\alpha}\right)
$$

Meyer-Vietoris Axiom: if $A_{1}, A_{2}$ are subcomplexes of $X$ with $X=\operatorname{int}\left(A_{1}\right) \cup \operatorname{int}\left(A_{2}\right)$, then for any $x_{1} \in F\left(A_{1}\right)$ and $x_{2} \in F\left(A_{2}\right)$ with $\left.x_{1}\right|_{A_{1} \cap A_{2}}=\left.x_{2}\right|_{A_{1} \cap A_{2}}$ there exists $y \in F(X)$ with $\left.y\right|_{A_{1}}=x_{1}$ and $\left.y\right|_{A_{2}}=x_{2}$. 
Lemma 3.1.5: If $F$ is a contravariant functor satisfying both the wedge and Meyer-Vietoris axioms, then $F\left(\left\{y_{0}\right\}\right)$ contains only one element for any one-point space $\left\{y_{0}\right\}$.

Proof of Lemma 3.1.5: Using the wedge axiom, we see that

$$
F\left(\left\{y_{0}\right\}\right)=F\left(\left\{y_{0}\right\} \vee\left\{y_{0}\right\}\right) \simeq F\left(\left\{y_{0}\right\}\right) \times F\left(\left\{y_{0}\right\}\right)
$$

where the map takes $v \in F\left(\left\{y_{0}\right\}\right)$ to the pair $(v, v)$. The only way this map is an isomorphism is if $F\left(\left\{y_{0}\right\}\right)$ is unitary, as required.

It is simple to check that any representable functors satisfy the properties above.

Proposition 3.1.6: For any $C W$ space $\left(Y, y_{0}\right)$, the functor $\left[-;\left(Y, y_{0}\right)\right]$ satisfies both wedge and Meyer-Vietoris axioms.

A proof of this result is seen in [Swi75, Proposition 9.1.

We now try to prove the "converse" of the result above. That is, from an arbitrary contravariant functor satisfying the properties above, define a suitable CW-complex $Y$ and $u \in F(Y)$, such that the map $T_{u}$ is an equivalence. We do this using the following strategy: first we try to satisfy the desired property on spheres, and then we try to extend it for all CW-complexes.

Definition 3.1.4: $\quad$ An element $u \in F(Y)$ is called $n$-universal if

$$
T_{u}:\left[\left(S^{q}, s_{0}\right) ;\left(Y, y_{0}\right)\right] \rightarrow F\left(S^{q}\right)
$$

is an isomorphism for all $q<n$ and an epimorphism if $q=n$. Such an $u$ is said to be universal if it is n-universal for all $n$.

For notational reasons, we say every element of $F\left(Y, y_{0}\right)$ is $(-1)$-universal.

Lemma 3.1.7: Let $f:\left(Y, y_{0}\right) \rightarrow\left(Y^{\prime}, y_{0}^{\prime}\right)$ with $u \in F(Y)$ and $u^{\prime} \in F\left(Y^{\prime}\right)$ n-universal elements such that $f^{*}\left(u^{\prime}\right)=u$. Then $f$ induces isomorphisms $f_{*}: \pi_{q}\left(Y, y_{0}\right) \rightarrow \pi_{q}\left(Y^{\prime}, y_{0}^{\prime}\right)$, for all $q<n$. In particular, if $u$ and $u^{\prime}$ are universal, then $f$ induces isomorphisms between all homotopy groups.

Proof of Lemma 3.1.7: For any $q<n$, we know there are isomorphisms $T_{u}: \pi_{q}\left(Y, y_{0}\right)=$ $\left[\left(S^{q}, s_{0}\right) ;\left(Y, y_{0}\right)\right] \simeq F\left(S^{q}\right)$ and $T_{u^{\prime}}: \pi_{q}\left(Y^{\prime}, y_{0}^{\prime}\right) \simeq F\left(S^{q}\right)$. Since $f^{*}\left(u^{\prime}\right)=u$, we have, for any $[\alpha] \in \pi_{q}\left(Y, y_{0}\right)$

$$
T_{u^{\prime}} \circ f_{*}[\alpha]=T_{u^{\prime}}[f \circ \alpha]=\alpha^{*} \circ f^{*}\left(u^{\prime}\right)=\alpha^{*}(u)=T_{u}[\alpha]
$$

Since both $T_{u}$ and $T_{u^{\prime}}$ are isomorphisms, the result follows.

Lemma 3.1.8: Given $\left(Y, y_{0}\right)$ and $u_{n} \in F(Y)$ an n-universal element, there is a $C W$-complex $Y^{\prime}$ obtained from $Y$ by attaching $(n+1)$-cells and an $(n+1)$-universal element $u_{n+1} \in F\left(Y^{\prime}\right)$ such that $\left.u_{n+1}\right|_{Y}=u_{n}$.

Since we are looking at attaching cells, the following result is very useful to prove the Lemma above: 
Proposition 3.1.9: $\quad$ For any map $f:\left(X, x_{0}\right) \rightarrow\left(Y, y_{0}\right)$ the sequence

$$
F(X) \stackrel{f^{*}}{\longleftarrow} F(Y) \stackrel{j^{*}}{\longleftarrow} F\left(Y \cup_{f} C X\right)
$$

is exact.

Proof of Proposition 3.1.9: First, since $f^{*} \circ j^{*}=(j \circ f)^{*}=0$, we know that $\operatorname{Im} j^{*} \subset \operatorname{ker} f^{*}$. On the other hand, let $y \in F(Y)$ such that $f^{*}(y)=0$. We think of $Y \cup_{f} C X$ as the union of the spaces $A_{1}=\left[0, \frac{1}{2}\right] \wedge X$ and $A_{2}=Y \cup_{f}\left[\frac{1}{2}, 1\right]^{+} \wedge X$. Then $A_{1} \cap A_{2}=\left\{\frac{1}{2}\right\} \times X \simeq X$ and $A_{2} \simeq Y$. Also, we see that the inclusion $X \simeq A_{1} \cap A_{2} \simeq A_{2}$ is essentially the map $f$.

Since $F\left(A_{2}\right) \simeq F(Y)$, we choose $y_{2} \in F\left(A_{2}\right)$ corresponding to $y$, and we choose $y_{1} \in F\left(A_{1}\right)$ to be the zero element. In $A_{1} \cap A_{2}$, we have

$$
\left.y_{2}\right|_{A_{1} \cap A_{2}}=f^{*}(y)=0=\left.y_{1}\right|_{A_{1} \cap A_{2}}
$$

Therefore, the Meyer-Vietoris principle assures us that there is an element $h \in F\left(Y \cup_{f} C X\right)$, such that $\left.h\right|_{Y}=y$, as required.

Let's move to the proof of Lemma 3.1.8.

Proof of Lemma 3.1.8: Since $u_{n} \in F(Y)$ is an $n$-universal element, we know that $T_{u_{n}}$ : $\left[\left(S^{q}, s^{0}\right),\left(Y, y_{0}\right)\right] \rightarrow F\left(S^{q}\right)$ is an isomorphism for $q<n$ and an epimorphism in $q=n$.

We construct $Y^{\prime}$ by attaching $(n+1)$-cells to $Y$ and guaranteeing that it has a $(n+1)$-universal element, such that $\left.u_{n+1}\right|_{Y}=u_{n}$. Since we only attach cells of a high dimension, we see that, for $q<n$, all maps $\left(S^{q}, s_{0}\right) \rightarrow\left(Y^{\prime}, y_{0}\right)$ are, by the Cellular Approximation Theorem, thought of as a map to $Y$ and therefore $T_{u_{n+1}}$ is an isomorphism on these dimensions. So we only worry about dimensions $q=n$ and $q=n+1$.

First, we will make sure that $T_{u_{n+1}}$ is an epimorphism on dimension $n+1$. To do that, we consider, for each element $\lambda \in F\left(S^{n+1}\right)$, an $(n+1)$-sphere, $S_{\lambda}^{n+1}$. Now we take the wedge sum $Y \vee \bigvee_{\lambda} S_{\lambda}^{n+1}$. From the wedge axiom, we know there is an element $v \in F\left(Y \vee \bigvee_{\lambda} S_{\lambda}^{n+1}\right)$ such that $\left.v\right|_{Y}=u_{n}$ and $\left.v\right|_{S_{\lambda}^{n+1}}=\lambda$. So this $v$ is enough to satisfy our surjection condition.

Now we look on how to make $T_{u_{n+1}}$ injective in dimension $n$ (since surjectivity is already guaranteed from the fact that $\left.\left.u_{n+1}\right|_{Y}=u_{n}\right)$. For each element $[\alpha] \in \pi_{n}\left(Y, y_{0}\right)$ such that $T_{u_{n}}[\alpha]=0$, we take $\xi_{\alpha}:\left(S^{n}, s_{0}\right) \rightarrow\left(Y, y_{0}\right)$ a representative of $[\alpha]$ and attach an $(n+1)$-cell $e_{\alpha}^{n+1}$ to $Y \vee \underset{\lambda}{\vee} S_{\lambda}^{n+1}$, with attaching map $\xi_{\alpha}$. This space will be our $Y^{\prime}$. Now all we need to do is obtain an $(n+1)$ universal element.

From an analogous argument to the one in Proposition 3.1.9, we see that, if $g: \bigvee_{\alpha} S_{\alpha}^{n} \rightarrow Y \vee$ $\bigvee_{\lambda} S_{\lambda}^{n+1}$ is the combination of all the attaching maps, then the following sequence

$$
F\left(\bigvee_{\alpha} S_{\alpha}^{n}\right) \stackrel{g^{*}}{\longleftarrow} F\left(Y \vee \bigvee_{\lambda} S_{\lambda}^{n+1}\right) \stackrel{j^{*}}{\longleftarrow} F(Y)
$$


is exact. Since $\left.v\right|_{S_{\alpha}^{n}}=\left.u_{n}\right|_{S_{\alpha}^{n}}=T_{u_{n}}(\alpha)=0$, for all $\alpha$, and the map $\left\{i_{\alpha}^{*}\right\}: \prod F\left(S_{\alpha}^{n}\right) \rightarrow F\left(\bigvee_{\alpha} S_{\alpha}^{n}\right)$ is an isomorphism, we see that $g^{*}(v)=0$. By exactness, there is an element $u_{n+1} \in F(Y)$ such that, when restricted to $Y \vee \bigvee_{\lambda} S_{\lambda}^{n+1}$, is precisely $v$. We show that such $u_{n+1}$ is the desired element.

If $T_{u_{n+1}}(\beta)=0$, for some $\beta \in \pi_{n}\left(Y^{\prime}, s_{0}\right)$, we know it is homotopic to an element $\alpha \in \pi_{n}\left(Y, y_{0}\right)$. Then, if $i: Y \rightarrow Y^{\prime}$ is the inclusion, $0=T_{u_{n+1}}(\beta)=T_{u_{n+1}}\left(i^{*}(\alpha)\right)=T_{u}(\alpha)$. But then there is a cell $e_{\alpha}^{n+1}$ in $Y^{\prime}$, making $\beta=i^{*}(\alpha)=0$. Hence, $T_{u_{n+1}}$ is injective in dimension $n$, and therefore, an isomorphism.

Finally, any element $\lambda \in F\left(S^{n+1}\right)$ is given by $\left.u_{n+1}\right|_{S_{\lambda}^{n+1}}=T_{u_{n+1}}\left(i_{\lambda}^{*}\right)$, which implies that $T_{u_{n+1}}$ is surjective in dimension $n+1$, as required.

Corollary 3.1.10: Given a $C W$-complex $\left(Y, y_{0}\right)$ and $v \in F(Y)$, there is a $C W$-complex $Y^{\prime}$ with $Y$ as a subcomplex and an universal element $u \in F\left(Y^{\prime}\right)$ with $\left.u\right|_{Y}=v$.

Proof of Lemma 3.1.10: What we do is apply the previous lemma recursively. First, we apply it on the CW-complex $\left(Y, y_{0}\right)$ and the $(-1)$-universal element $v \in F(Y)$. Then we get a CW-complex $Y_{0}$, obtained from $Y$ by attaching 1-cells and a 0-universal element $u_{0} \in F\left(Y_{0}\right)$. Recursively, we obtain a sequence $Y \subset Y_{0} \subset Y_{1} \subset \ldots$ in which each $Y_{n+1}$ is obtained from $Y_{n}$ by attaching $(n+1)$-cells, and $u_{n} \in F\left(Y_{n}\right)$ is an $n$-universal element, for all $n$.

Let $Y^{\prime}=\cup Y_{n}$ with the weak topology. Using the properties of the inverse limit and both the wedge and Meyer-Vietoris arguments, it is simple to show that

$$
\left\{i_{n}^{*}\right\}: F\left(Y^{\prime}\right) \rightarrow \operatorname{inv} \lim F\left(Y_{n}\right)
$$

is surjective, and therefore, there must be an element $u \in F(Y)$ such that $\left.u\right|_{Y_{n}}=u_{n}$, for all $n$. Then, for each $n$ and $q$, a commutative diagram

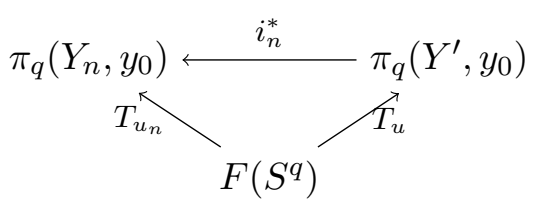

Since $Y^{\prime}$ is obtained from $Y_{n}$ by attaching cells of dimension higher then $n+1$, we know that the $i_{n}^{*}$ induces an isomorphism in the diagram above, for all $q<n$. This implies that $T_{u}$ is an isomorphism for all $q<n$. Since this argument is valid for every $n$, we get that $u$ is universal.

Corollary 3.1.11: $\quad$ There exists a $C W$-complex $\left(Y, y_{0}\right)$ and a universal element $u \in F(Y)$.

Proof of Lemma 3.1.11: This follows directly from Lemma 3.1.10. For instance, we can apply the previous result to $Y=\left\{y_{0}\right\}$ and $v \in F\left(y_{0}\right)$, the only element of this set (see Lemma 3.1.5).

What we now show is that, if $u$ is an universal element, then $T_{u}$ induces an isomorphism not only in spheres but in all CW-complexes. The strategy to do that is very similar to the idea used 
to proof Whitehead's Theorem (see [Swi75], Theorem 6.32):

Lemma 3.1.12: $\quad$ Let $\left(Y, y_{0}\right)$ be a $C W$-complex with universal element $u \in F(Y)$. If $\left(X, A, x_{0}\right)$ is a CW-pair, a cellular map $g:\left(A, x_{0}\right) \rightarrow\left(Y, y_{0}\right)$ and $v \in F(X)$ such that $\left.v\right|_{A}=g^{*}(u)$, then there is a cellular map $h:\left(X, x_{0}\right) \rightarrow\left(Y, y_{0}\right)$ such that $\left.h\right|_{A}=g$ and $v=h^{*}(u)$.

Proof of Lemma 3.1.12: Let $T$ be the space obtained from $\left(I^{+} \wedge A\right) \vee X \vee Y$ by identifying $[0, a] \in I^{+} \wedge A$ with $a \in X$ and $[1, a] \in I^{+} \wedge A$ to $g(a) \in Y$.

We consider in $T$ the two following subcomplexes

$$
\begin{aligned}
& A_{1}=\left(\left[0, \frac{1}{2}\right]^{+} \wedge A\right) \cup X \\
& A_{2}=\left(\left[\frac{1}{2}, 1\right]^{+} \wedge A\right) \cup Y
\end{aligned}
$$

We see that $T=A_{1} \cup A_{2}$ and that $A_{1} \cap A_{2} \simeq A$. Also, there is an obvious strong deformation retract $r: A_{1} \rightarrow X$, which implies there is an element $x_{1} \in F\left(A_{1}\right) \simeq F(X)$, such that $\left.x_{1}\right|_{X}=v$. Analogously, we see that $Y$ is a strong deformation retract of $A_{2}$ and therefore there is $x_{2} \in$ $F\left(A_{2}\right)$, such that $\left.x_{2}\right|_{Y}=u$.

Using Lemma 3.1.10, we construct a CW complex $Y^{\prime}$ containing $T$ as a subcomplex, with a universal element $u^{\prime} \in F(T)$, such that $\left.u^{\prime}\right|_{T}=w$.

If $j: Y \rightarrow Y^{\prime}$ is the inclusion, we see that $j^{*}\left(u^{\prime}\right)=\left.u^{\prime}\right|_{Y}=u$ and therefore, by Lemma 3.1.7. $j^{*}: \pi_{*}\left(Y, y_{0}\right) \rightarrow \pi_{*}\left(Y^{\prime}, y_{0}\right)$ is an isomorphism. In particular, by Whitehead's Theorem 1.3.6, $Y$ and $Y^{\prime}$ are homotopy equivalent.

On the other hand, if $i: X \rightarrow Y^{\prime}$ is the inclusion, we know that $\left.i\right|_{A}$ is homotopic to $j \circ g$, since the composition $H: I^{+} \wedge A \hookrightarrow T \hookrightarrow Y^{\prime}$ is a basepoint preserving homotopy from $\left.i\right|_{A}$ to $j \circ g$.

We now use the fact that all inclusion of a subcomplex $A \hookrightarrow X$ is a cofibering (see [Swi75], Proposition 6.5), to find a homotopy $I^{+} \wedge X \rightarrow Y^{\prime}$ extending $H$, satisfying: the restriction of this homotopy to $\{1\}^{+} \wedge X$ gives us a map $\tilde{g}: X \rightarrow Y^{\prime}$ with $\left.\tilde{g}\right|_{A}=j \circ g$ and $\tilde{g} \simeq i$.

Then we have produced maps which can be arranged in the following commutative diagram:

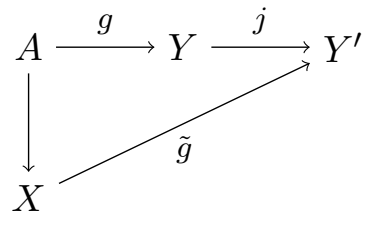

Since $Y$ and $Y^{\prime}$ are homotopy equivalent and $(X, A)$ is a CW-pair, we construct a map $h$ : $X \rightarrow Y$ with $\left.h\right|_{A}=g$ and $j \circ h \simeq \tilde{g} \simeq i$ (see [Swi75], Theorems 6.29 and 6.30). Moreover, $h^{*}(u)=h^{*} \circ j^{*}\left(u^{\prime}\right)=(j \circ h)^{*}\left(u^{\prime}\right)=i^{*}\left(u^{\prime}\right)=v$, as required.

Theorem 3.1.13: (Brown's Representability Theorem) If F is a contravariant functor from the category of pointed $C W$-complexes to the category of pointed topological spaces satisfying the wedge and Mayer-Vietoris axioms, then there exists a classifying space and a universal 
element $u \in F\left(Y, y_{0}\right)$, such that

$$
T_{u}:\left[-;\left(Y, y_{0}\right)\right] \rightarrow F(-)
$$

is a natural equivalence.

Proof of Theorem 3.1.13: From Corollary 3.1.11, we know there is a CW-complex $\left(Y, y_{0}\right)$ and an universal element $u \in F(Y)$. What we show is that, for an arbitrary $\left(X, x_{0}\right)$, the map $T_{u}:\left[\left(X, x_{0}\right) ;\left(Y, y_{0}\right)\right] \rightarrow F(X)$ is bijective.

For surjectivity, fix an arbitrary $v \in F(X)$. We apply Lemma 3.1.12 with $A=\left\{x_{0}\right\}, g$ the map constant equals $y_{0}$ and $v$ the fixed element on $F(X)$. Then we have proven there is a map $h:\left(X, x_{0}\right) \rightarrow\left(Y, y_{0}\right)$ with $v=h^{*}(u)=T_{u}(h)$. Therefore, $T_{u}$ is surjective.

On the other hand, let $g_{0}, g_{1}:\left(X, x_{0}\right) \rightarrow\left(Y, y_{0}\right)$ be such that $T_{u}\left[g_{0}\right]=T_{u}\left[g_{1}\right]$. By the Cellular Approximation Theorem, we assume $g_{0}$ and $g_{1}$ to be cellular maps and thus apply the Lemma 3.1 .12 on the spaces $X^{\prime}=I^{+} \wedge X, A^{\prime}=\{0,1\}^{+} \wedge X$ with $g: A \rightarrow Y$ defined as $g[0, x]=g_{0}(x)$ and $g[1, x]=g_{1}(x)$. Then, if $p: X^{\prime} \rightarrow X$ is the projection, we take $v=p^{*} \circ g_{0}^{*}(u) \in F\left(X^{\prime}\right)$. This makes

$$
\begin{aligned}
& \left.v\right|_{\{0\}^{+} \wedge X}=g_{0}^{*}(u)=\left.g^{*}(u)\right|_{\{0\}^{+} \wedge X} \\
& \left.v\right|_{\{1\}^{+} \wedge X}=g_{0}^{*}(u)=T_{u}\left[g_{0}\right]=T_{u}\left[g_{1}\right]=g_{1}^{*}(u)=\left.g^{*}(u)\right|_{\{1\}^{+} \wedge X}
\end{aligned}
$$

Then $g^{*} u=\left.v\right|_{A}$ and therefore, applying Lemma 3.1.12, we see there is a map $h: X^{\prime} \rightarrow Y$ with $\left.h\right|_{A}=g$. This means that $h$ is a homotopy between $g_{0}$ and $g_{1}$, as required.

Theorem 3.1.14: $\quad$ Let $F$ and $F^{\prime}$ be two contravariant functors from the category of pointed $C W$-complexes to the category of pointed topological spaces with classifying spaces $\left(Y, y_{0}\right)$ and $\left(Y^{\prime}, y_{0}^{\prime}\right)$ respectively. If $T: F \rightarrow F^{\prime}$ is a natural transformation, then there is a map $f:\left(Y, y_{0}\right) \rightarrow$ $\left(Y^{\prime}, y_{0}^{\prime}\right)$ unique up to homotopy, such that the diagram

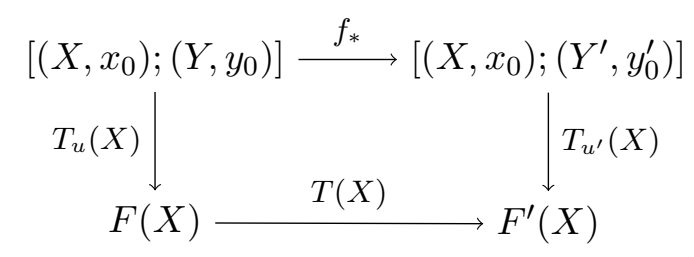

commutes for all $\left(X, x_{0}\right)$.

Proof of Theorem 3.1.14: Let $f:\left(Y, y_{0}\right) \rightarrow\left(Y^{\prime}, y_{0}^{\prime}\right)$ be such that

$$
\left(T(Y) \circ T_{u}(Y)\right)\left[I d_{y}\right]=T_{u^{\prime}}(Y)[f]
$$

which exists because $T_{u^{\prime}}$ is an isomorphism. Then, for all $\left(X, x_{0}\right)$ and $\alpha \in\left[\left(X, x_{0}\right) ;\left(Y, y_{0}\right)\right]$, by 
the naturality of $T$,

$$
\begin{aligned}
T_{u^{\prime}} \circ f_{*}[\alpha] & =T_{u^{\prime}}[f \circ \alpha]=(f \circ \alpha)^{*}\left(u^{\prime}\right)=\alpha^{*} T_{u^{\prime}}(Y)(f) \\
& \left.\left.=\alpha^{*} \circ(T(Y)) \circ T_{u}(Y)\right)\left[I d_{y}\right]\right)=T(X) \circ \alpha^{*} T_{u}(Y)\left[I d_{y}\right]=T(X) \circ T_{u}[\alpha]
\end{aligned}
$$

In particular, it follows from the previous result that the classifying space is determined up to homotopy equivalence.

Therefore, we have proven that every covariant functor satisfying the wedge and Mayer-Vietoris axioms is representable. Which, in particular, indicates that constructing a cohomology theory is somehow equivalent to choosing a well-behaved sequence of based spaces $\left\{\left(K_{n}, k_{n}\right)\right\}$ and defining the cohomology groups as the functors represented by such spaces.

With this in hand, we can study the values of a cohomology theory on a space $\left(X, x_{0}\right)$ by understanding the homotopy classes of maps $\left(X, x_{0}\right) \rightarrow\left(K_{n}, k_{n}\right)$. For instance, if we look at the values of one such functor on spheres, we get:

$$
\left[\left(S^{n}, s_{0}\right),\left(K_{n}, k_{n}\right)\right]=\pi_{n}\left(K_{n}, k_{n}\right)
$$

Therefore, if we want this functor to be well-behaved on spheres, it makes sense to choose as the classifying space a $K$ for which we know the homotopy groups.

It immediately comes to mind the idea of studying functors which are represented by EilenbergMacLane spaces $K(\pi, n)$ with $\pi$ abelian. We know that these are extremely well-behaved when evaluated on spheres:

$$
H^{n}\left(S^{m}\right)=\pi_{m}(K(\pi, n))= \begin{cases}\pi & \text { if } n=m \\ 0 & \text { otherwise }\end{cases}
$$

Also, if $\pi$ is abelian, we know that $K(\pi, n)=\Omega K(\pi, n+1)=\Omega^{2} K(\pi, n+2)$, from which we conclude that functors which are represented by Eilenberg-MacLane spaces take values on abelian groups.

From all such aspects and for the simplicity of $K(\pi, n)$ 's, it is natural to enquire whether the functors represented by them will be fairly computable on CW-complexes. This is indeed the case, which is expressed in the following surprising result:

Theorem 3.1.15: $\quad$ For $C W$-complexes $\left(X, x_{0}\right)$, abelian groups $\pi$, and integers $n \geq 0$, there are natural isomorphisms

$$
F: \tilde{H}_{C W}^{n}(X ; \pi) \simeq\left[\left(X, x_{0}\right),(K(\pi, n), *)\right]
$$

where $\tilde{H}_{C W}^{n}$ is the ordinary reduced cellular cohomology of $X$.

Proof of Theorem 3.1.15: From the results developed in section 1.3 , we assume that $K(\pi, n)$ is a space built from a wedge of $n$-cells by attaching cells of higher dimensions. Then, to each 
cocycle $c \in H_{C W}^{n}(X, \pi)$, we associate a map $f_{c}^{\prime}: X^{n} \rightarrow K(\pi, n)$ with the following properties:

- $\left.f_{c}^{\prime}\right|_{X^{n-1}}$ is the map constant to the 0 -cell of $K(\pi, n)$;

- for each cell $e_{\alpha}^{n}$ of $X, f_{c}^{\prime}$ maps $e_{\alpha}^{n}$ to a representative $S^{n} \rightarrow K(\pi, n)$ of $c\left(e_{\alpha}^{n}\right) \in \pi_{n}(K(\pi, n)) \simeq$ $\pi$.

Since $c$ is a cocycle, we know that the boundaries of all $(n+1)$-cells of $X$ are mapped to the 0 element and, therefore, using the results from Obstruction Theory, we extend $f_{c}^{\prime}$ to $X^{n+1}$. Moreover, since $\pi_{k}(K(\pi, n))=0$, for $k>n$, we know that we can extend $f_{c}^{\prime}$ to all of $X$, obtaining a map $f_{c}: X \rightarrow K(\pi, n)$.

The map $F$ between the two functors is the association $c \rightarrow\left[f_{c}\right]$.

It is simple to see that $F$ is injective, since distinct elements of $H^{n}(X, \pi)$ produce maps which are not homotopic on $X^{n}$, by construction.

Moreover, since any class in $[X, K(\pi, n)]$ is represented by a cellular map $f: X \rightarrow K(\pi, n)$, the map which assigns to each $n$-cell $e_{\alpha}^{n}$ of $X$ the class $\left[f\left(e_{\alpha}^{n}\right)\right] \in \pi_{n}(K(\pi, n)) \simeq \pi$ will be a representative of an element $c \in H^{n}(X, \pi)$, such that $F(c)=[f]$.

Using the group structure in $[X, K(\pi, n)]$ given by the $H$-group structure of $K(\pi, n)$, we see that such a bijection is indeed a homomorphism and thus an isomorphism.

The theorem above then guarantees that the usual classical $n$-th cohomology group is represented by an Eilenberg-MacLane space and, in particular, proves homotopy equivalence of the original theory.

With so many cohomology theories being created and proved to be equivalent, Eilenberg and Steenrod decided to make a list of properties that all those theories satisfied and think of it as a set of axioms for a cohomology theory. In the article Axiomatic approach to homology theory ([ES45]), they started the first axiomatic approach to homology and cohomology.

Definition 3.1.5: $\quad$ A reduced cohomology theory satisfying the Eilenberg-Steenrod axioms is a sequence $\tilde{F}^{*}=\left\{\tilde{F}^{n}\right\}$ of contravariant functors from the category of pointed $C W$-complexes to the category of abelian groups, satisfying the following axioms:

1R - Homotopy Invariance: if $f, g:\left(X, X_{0}\right) \rightarrow\left(Y, y_{0}\right)$ are homotopic via a basepoint preserving homotopy, then the induced morphisms $f^{*}, g^{*}: \tilde{F}^{*}\left(Y, y_{0}\right) \rightarrow \tilde{F}^{*}\left(X, x_{0}\right)$ are equal.

2R - Exactness: If $x_{0} \in A \subset X$ then we have an exact sequence of graded abelian groups

$$
\tilde{F}^{n}(X \cup C A, *) \longrightarrow \tilde{F}^{n}\left(X, x_{0}\right) \longrightarrow \tilde{F}^{n}\left(A, x_{0}\right)
$$

where the arrows are induced by the inclusions $A \rightarrow X$ and $X \rightarrow X \cup C A$.

3R - Suspension: For each integer n, there is a natural isomorphism

$$
\tilde{F}^{n}\left(X, x_{0}\right) \rightarrow \tilde{F}^{n}(S X, *)
$$


4R - Additivity: For any set of pointed spaces $\left(X_{i}, x_{i}\right)$, the inclusions $\left(X_{i}, x_{i}\right) \rightarrow\left(\vee_{i} X_{i}, *\right)$ induce an isomorphism

$$
\tilde{F}^{n}\left(\vee_{i} X_{i}, *\right) \rightarrow \prod_{i} \tilde{F}^{n}\left(X_{i}, x_{i}\right)
$$

5R - Dimension: $\tilde{F}^{n}\left(S^{0}\right)=0$, for all $n \geq 1$. The group $\tilde{F}^{0}\left(S^{0}\right)$ is called the coefficient group of $\tilde{F}$.

This gives us the definition of what is today called ordinary reduced cohomology. Analogously, Eilenberg and Steenrod also defined the following:

Definition 3.1.6: $\quad$ An unreduced cohomology theory on $C W$-complexes satisfying the EilenbergSteenrod axioms is a sequence $F^{*}=\left\{F^{n}\right\}$ of contravariant functors from the homotopy category of $C W$-pairs to the category of abelian groups, together with natural transformation $\delta: F^{n}(A, \emptyset) \rightarrow$ $F^{n+1}(X, A)$, satisfying the following axioms:

1U - Homotopy Invariance: if $f:(X, A) \rightarrow(Y, B)$ is a homotopy equivalence of pairs then

$$
f^{*}: F^{*}(Y, B) \rightarrow F^{*}(X, A)
$$

is an isomorphism.

2U - Exactness: For all pairs $(X, A)$, the maps induced by the inclusions $A \rightarrow X$ and $(X, \emptyset) \rightarrow$ $(X, A)$ give rise to an exact sequence:

$$
\cdots \longrightarrow F^{n}(X, A) \longrightarrow F^{n}(X, \emptyset) \longrightarrow F^{n}(A, \emptyset) \stackrel{\delta}{\longrightarrow} F^{n+1}(X, A) \longrightarrow \cdots
$$

3U - Excision: If $A, B \subset X$ are such that $X=\operatorname{int}(A) \cup \operatorname{int}(B)$, then the inclusion $(A, A \cap B) \rightarrow$ $(X, B)$ induces an isomorphism

$$
F^{n}(X, B) \rightarrow F^{n}(A, A \cap B)
$$

4U - Additivity: If $(X, A)$ is the disjoint union of a set of pairs $\left(X_{i}, A_{i}\right)$, then the inclusions $\left(X_{i}, A_{i}\right) \rightarrow(X, A)$ induce an isomorphism

$$
F^{n}(X, A) \rightarrow \prod_{i} F^{n}\left(X_{i}, A_{i}\right)
$$

5U -Dimension: $F^{n}$ (point) $=0$, for all $n \geq 1$. The group $F^{0}$ (point) is called the coefficient group of $F$.

Using such definitions, we see that, by fixing some abelian group $\pi$ and setting $\tilde{F}^{n}\left(X, x_{0}\right)=$ $\left[\left(X, x_{0}\right) ;(K(\pi, n), *)\right]$, we get a generalised reduced cohomology theory which is equal to the original cellular cohomology. Some of the properties which make $\tilde{F}^{n}$ into a cohomology theory 
are extremely simple to see, for instance, the dimension axiom:

$$
H^{n}(\text { point })= \begin{cases}\pi & \text { if } n=0 \\ 0 & \text { otherwise }\end{cases}
$$

and the suspension axiom, which follows directly from the relation $K(\pi, n) \simeq \Omega K(\pi, n+1)$ and Corollary 3.1.4.

The properties listed on the above definition were shown to be satisfied by all the cohomology theories to that date and these were all eventually shown to equal the cellular cohomology on CW-complexes. In fact, in the article [ES45], Eilenberg and Steenrod show that this will happen for any theory satisfying such axioms:

Theorem 3.1.16: (Uniqueness Theorem) Any unreduced (resp. reduced) cohomology theory satisfying the Eileinberg-Steenrod axioms and coefficient group $G$ agrees with the cellular cohomology (resp. reduced cohomology) with coefficients in $G$ on $C W$-complexes.

The proof of this theorem relies strongly on the dimension axiom, from which we have a relation between the functors. It is from this relation that we construct the equivalence between the theories.

Therefore, it made sense to broaden the definition of cohomology by dropping the dimension axiom.

Definition 3.1.7: A generalised reduced cohomology theory is a sequence $\tilde{F}^{*}=\left\{\tilde{F}^{n}\right\}$, $n \in \mathbb{Z}$, of contravariant functors from the category of pointed $C W$-complexes to the category of abelian groups satisfying homotopy invariance (1R), exactness (2R), suspension (3R) and additivity (4R) axioms.

Definition 3.1.8: A generalised unreduced cohomology theory is a sequence $F^{*}=\left\{F^{n}\right\}$, $n \in \mathbb{Z}$, of covariant functors from the category of $C W$-pairs to the category of abelian groups, together with a natural transformation $\delta: F^{n}(A, \emptyset) \rightarrow F^{n+1}(X, A)$, satisfying homotopy invariance $(1 U)$, exactness (2U), excision (3U) and additivity (4U) axioms.

When studying ordinary homology and cohomology, one encounters the terms "reduced" and "unreduced", however it might not have been clear that they actually defined functors from different categories. When studying generalised cohomology, it is important to make such definitions clear and we see that, parallel to what we had for ordinary cohomology, we have relations between the two concepts:

Theorem 3.1.17: $\quad$ A reduced generalised cohomology theory $\tilde{F}^{*}$ on based $C W$ complexes determines and is determined by an unreduced generalised cohomology theory $F^{*}$ on $C W$ pairs.

The detailed proof of this result is seen in Chapter 7 of [Swi75]. However, we give an idea of the relation between such categories: if $\tilde{F}^{*}$ be a reduced cohomology theory, we define $F^{*}$ on a pair $(X, A)$, by setting $F^{*}(X, A)=\tilde{F}^{*}(X \cup C A, *)$. It is simple to see that such $F^{*}$ yields an unreduced generalised cohomology theory. On the other hand, from an unreduced cohomology theory $F^{*}$, we defined $\tilde{F}^{*}$ by setting $\tilde{F}^{*}\left(X, x_{0}\right)=F\left(X,\left\{x_{0}\right\}\right)$ and it is simple to see that this $\tilde{F}^{*}$ 
is indeed a cohomology theory. This identification gives us an equivalence between the categories of reduced and unreduced generalised cohomology theories. Moreover, we have the following:

Theorem 3.1.18: $\quad$ Let $F^{*}$ be an unreduced cohomology theory and $\tilde{F}^{*}$ the corresponding reduced cohomology theory. For every pointed space $\left(X, x_{0}\right)$, there is an identification

$$
F^{n}(X) \simeq \tilde{F}^{n}\left(X, x_{0}\right) \oplus F^{n}\left(x_{0}\right)
$$

where $F^{n}(X)=F^{n}(X, \emptyset)$ and $F^{n}\left(x_{0}\right)=F^{n}\left(\left\{x_{0}\right\}, x_{0}\right)$.

Proof of Theorem 3.1.18: From the relation $\tilde{F}\left(X, x_{0}\right)=F\left(X,\left\{x_{0}\right\}\right)$ and the exactness axiom $(2 \mathrm{U})$, we obtain an exact sequence

$$
\cdots \rightarrow F^{n-1}\left(x_{0}\right) \rightarrow \tilde{F}^{n}\left(X, x_{0}\right) \rightarrow F^{n}(X) \rightarrow F^{n}\left(x_{0}\right) \rightarrow \tilde{F}^{n+1}\left(X, x_{0}\right) \rightarrow \ldots
$$

Since the composite $x_{0} \hookrightarrow X \rightarrow x_{0}$ is the identity, the morphism $F^{n}(X) \rightarrow F^{n}\left(x_{0}\right)$ has a section and so, in particular, is an epimorphism. By exactness of the sequence above, we see that the connecting homomorphism is trivial and therefore, for each $n$, we have a short exact sequence

$$
0 \rightarrow \tilde{F}^{n}\left(X, x_{0}\right) \rightarrow F^{n}(X) \rightarrow F^{n}\left(x_{0}\right) \rightarrow 0
$$

in which the right map admits a section. Therefore, this sequence splits and we get the desired result.

These new definitions lead mathematicians to look for cohomology theories which do not satisfy the dimension axiom using similar methods to the ones developed above. One important result on this matter comes from the fact that a reduced generalised cohomology theory $\tilde{F}^{*}=\left\{\tilde{F}^{n}\right\}$ is, by definition, composed by contravariant functors $\tilde{F}^{n}$, which satisfy both wedge and Meyer-Vietoris axioms. Then, by Brown's Representability Theorem, we obtain:

Theorem 3.1.19: For any reduced generalised cohomology theory $\tilde{F}^{*}=\left\{\tilde{F}^{n}\right\}$, there is a sequence of pointed $C W$-complexes $\left(E_{n}, e_{n}\right)$, such that each functor $F^{n}$ is represented by $\left(E_{n}, e_{n}\right)$.

We have provided an example of this when we proved that the ordinary reduced cohomology theory is represented by Eilenberg-MacLane spaces. When we mentioned this equivalence, we commented that some of the main reasons we could construct a cohomology theory from the $K(\pi, n)$ 's was the $H$-group structure of the loop space and the relation $K(\pi, n) \simeq \Omega K(\pi, n+1)$. What we now see is that, for any reduced generalised cohomology theory, the corresponding classifying spaces must have a similar relation.

If $\tilde{F}^{*}=\left\{\tilde{F}^{n}\right\}$ is a reduced generalised cohomology theory with corresponding classifying spaces 
$\left(E_{n}, e_{n}\right)$, then by the suspension axiom $(4 \mathrm{R})$ we have

$$
\begin{aligned}
{\left[\left(X, x_{0}\right),\left(E_{n}, e_{n}\right)\right] } & =\tilde{F}^{n}\left(X, x_{0}\right) \\
& \simeq F^{n+1}(S X, *) \\
& \simeq\left[\left(S X, x_{0}\right),\left(E_{n+1}, e_{n+1}\right)\right] \\
& \simeq\left[\left(X, x_{0}\right),\left(\Omega\left(E_{n+1}, e_{n+1}\right), \omega\right)\right]
\end{aligned}
$$

From what we discussed on Theorem 3.1.14 this natural equivalence must be induced by a homotopy equivalence

$$
\varepsilon_{n}:\left(E_{n}, e_{n}\right) \rightarrow\left(\Omega\left(E_{n+1}, e_{n+1}\right), \omega\right)
$$

This gives rise to the notion of Spectra:

Definition 3.1.9: A spectrum $E$ is a sequence of $C W$-complexes $\left(E_{n}, e_{n}\right), n \in \mathbb{Z}$, with base point preserving weak homotopy equivalences $\varepsilon_{n}: E_{n} \rightarrow \Omega E_{n+1}$.

What we showed is that, for any generalised reduced cohomology theory $\tilde{F}^{*}=\left\{\tilde{F}^{n}\right\}$, each $\tilde{F}^{n}$ is represented by a CW complex $\left(E_{n}, e_{n}\right)$, such that the collection of the $E_{n}$ 's forms a spectrum.

Conversely, we show that any collection of functors $K^{n}(-)=\left[-;\left(E_{n}, e_{n}\right)\right]$, where the collection of the $E_{n}$ 's forms a spectrum, gives rise to a generalised reduced cohomology theory (see [Swi75], Chapter 8).

A first immediate idea is to construct a spectrum from a CW-complex $\left(X, x_{0}\right)$ by taking successive iterations of the loop space:

$$
\ldots, \Omega^{2} X, \Omega X, X
$$

Indeed, in some cases, this is enough to produce a spectrum:

Example 3.1.4: $\quad$ The Eilenberg-MacLane Spectrum: for a fixed abelian group $\pi$, the sequence of spaces $K(\pi, n)$ forms a spectrum, which generates the ordinary cohomology theory.

At this point, we see how our spaces $B O$ and $B U$ fit perfectly to produce a well-behaved cohomology theory. First of all because representable functors evaluated on spheres yield the homotopy groups of the classifying spaces, then since Bott's Periodicity Theorem gives us a precise account of the homotopy groups of $B O$ and $B U$, these are very good options to define representable functors. However, the most important aspect of these spaces is that Bott's Periodicity Theorem also showed us that the sequences of their successive loop spaces are periodic, and, therefore, they can be used to construct a spectrum and a reduced cohomology theory.

This motivates the definition of the functors

$$
\widetilde{K O}\left(X, x_{0}\right)=\left[\left(X, x_{0}\right) ;(B O, *)\right] \quad \widetilde{K}\left(X, x_{0}\right)=\left[\left(X, x_{0}\right) ;(B U, *)\right]
$$

and the construction of the reduced generalised cohomology theories $\widetilde{K O}^{*}$ and $\widetilde{K}^{*}$ by setting, 
for every $n \in \mathbb{Z}$,

$$
\begin{aligned}
\widetilde{K O}^{8 n+i}\left(X, x_{0}\right) & =\left[\left(X, x_{0}\right) ;\left(\Omega^{i}(B O), \omega\right)\right]=\widetilde{K O}\left(S^{i} X, *\right) & \text { for } i=0,1, \ldots, 7 \\
\widetilde{K}^{2 n+i}\left(X, x_{0}\right) & =\left[\left(X, x_{0}\right) ;\left(\Omega^{i}(B U), \omega\right)\right]=\widetilde{K}\left(S^{i} X, *\right) & \text { for } i=0,1
\end{aligned}
$$

which are periodic and when evaluated in $S^{0}$ yield:

$$
\begin{array}{rlr}
\widetilde{K O}^{8 n+i}\left(S^{0}, s_{0}\right) & =\left\{\mathbb{Z}, \mathbb{Z}_{2}, \mathbb{Z}_{2}, 0, \mathbb{Z}, 0,0,0\right\} & \text { for } i=0,1, \ldots, 7 \\
\widetilde{K}^{2 n+i}\left(S^{0}, s_{0}\right) & =\{\mathbb{Z}, 0\} & \text { for } i=0,1
\end{array}
$$

All these properties of the functors $\tilde{K} O$ and $\tilde{K}$ make them much easier to work with, for instance:

Example 3.1.5: We show that $\tilde{K}^{0}\left(\mathbb{C} P^{n}\right) \simeq \bigoplus_{i=0}^{n} \mathbb{Z}$ and $\tilde{K}^{1}\left(\mathbb{C} P^{n}\right)=0$, for all $n$.

We proceed by induction: Since $\mathbb{C} P^{0}$ is a point, we know that $\tilde{K}^{0}\left(\mathbb{C} P^{0}\right) \simeq \mathbb{Z}$ and

$\tilde{K}^{1}\left(\mathbb{C} P^{n}\right)=0$. Applying the exactness axiom (2R) to the inclusions $\mathbb{C} P^{n-1} \subset \mathbb{C} P^{n}$ and recalling that $\mathbb{C} P^{n} / \mathbb{C} P^{n-1}=S^{2 n}$, we have

$$
\cdots \rightarrow \underbrace{\tilde{K}^{-3}\left(\mathbb{C} P^{n-1}\right)}_{0} \rightarrow \underbrace{\tilde{K}^{-2}\left(S^{2 n}\right)}_{\mathbb{Z}} \rightarrow \tilde{K}^{-2}\left(\mathbb{C} P^{n}\right) \rightarrow \tilde{K}^{-2}\left(\mathbb{C} P^{n-1}\right) \rightarrow \underbrace{\tilde{K}^{-1}\left(S^{2 n}\right)}_{0} \rightarrow \cdots
$$

and

$$
\cdots \rightarrow \underbrace{\tilde{K}^{-3}\left(S^{2 n}\right)}_{0} \rightarrow \tilde{K}^{-3}\left(\mathbb{C} P^{n}\right) \rightarrow \tilde{K}^{-3}\left(\mathbb{C} P^{n-1}\right) \rightarrow \rightarrow \ldots
$$

Using the induction hypothesis and the relation $\tilde{K}^{i}\left(S^{2} n\right)=\tilde{K}^{2 n+i}($ point $)$, we get that $\tilde{K}^{0}\left(\mathbb{C} P^{n}\right) \simeq$ $\bigoplus_{i=0}^{n} \mathbb{Z}$ and $\tilde{K}^{1}\left(\mathbb{C} P^{n}\right)=0$

Some simple computations also give us the unreduced analogues to the functors $\widetilde{K O}$ and $\tilde{K}$, which are given by

$$
K O(X)=[X, B O \times \mathbb{Z}] \quad K(X)=[X, B U \times \mathbb{Z}]
$$

and which generate the associated unreduced generalised cohomologies $K O *$ and $K *$, defined by

$$
\begin{aligned}
K O^{8 n+i}(X) & =\left[X ;\left(\Omega^{i}(B O \times \mathbb{Z}), \omega\right)\right]=K O\left(S^{i} X, *\right) & \text { for } i=0,1, \ldots, 7 \\
K^{2 n+i}(X) & =\left[X ;\left(\Omega^{i}(B U \times \mathbb{Z}), \omega\right)\right]=K\left(S^{i} X, *\right) & \text { for } i=0,1
\end{aligned}
$$

These cohomology theories compose what is called K-Theory and its periodicity is one of the main assets of its computability. However, the greatness of K-Theory is only seen by understanding its geometrical interpretation, which we discuss in the next section. 
As a final remark, we would like to comment on the question of whether there is an extension of the uniqueness theorem in this context, or more generally, how those abundantly many cohomology theories are related. This matter led Atiyah and Hirzebruch to produce a spectral sequence which was first written for K-Theory, but which is clearly valid for all cohomology theories.

Theorem 3.1.20: Let $F^{*}$ be a cohomology theory. Then there is a spectral sequence with $E_{2}$-term:

$$
E_{2}^{p, q}=H^{p}\left(P ; F^{q}(\text { point })\right)
$$

which converges to a graded group associated to $F^{*}(P)$.

Where $H^{p}$ denotes the classical cohomology.

Example 3.1.6: Let $F^{*}$ be a cohomology theory which satisfies the dimension axiom, i.e. $F^{0}$ (point $)=\pi$ for some abelian group $\pi$ and $F^{n}$ (point $)=0$ for all non-zero $n$. Then, 3.1.20 give us a spectral sequence converging to $F^{*}(P)$, whose second page has only one non-trivial row:

$$
E_{2}^{p, 0}=H^{p}(P ; \pi)
$$

which implies that this second page is equal to page $E^{\infty}$, and therefore, yields that $F^{p}(P)=$ $H^{p}(P ; \pi)$, which is the uniqueness theorem.

Therefore, we think of 3.1 .20 as a generalization of the uniqueness theorem.

\subsection{The Geometric Approach to K-Theory}

The geometrical idea behind K-Theory came from the mission of classifying vector bundles on a space, or in other words, understanding when two vector bundles on a topological space are essentially the same.

We start this exploration by looking at real vector bundles.

We have the definition of when two vector-bundles $E$ and $E^{\prime}$ over $X$ are isomorphic: when there exists a map $\varphi: E \rightarrow E^{\prime}$ which preserves fibers and induces a linear isomorphism $\varphi_{x}: E_{x} \rightarrow E_{x}^{\prime}$ on the fibers over $x \in X$. Then a first interesting idea to the matter of classifying vector bundles would be to look at the isomorphism classes of $n$-dimensional vector bundles over $X$ with the trivial bundle as the base point. This space is denoted $\operatorname{Vect}_{n}(X)$ and we can easily turn this association into a functor with the following definition: given an $n$-bundle $E$ over $X$ and a map $f: X^{\prime} \rightarrow X$, we define $f^{*}(E) \in \operatorname{Vect}_{n} X^{\prime}$ as the pullback bundle of $E$ over $f$.

This method gives us, for each non-negative integer $n$, a covariant functor $V e c t_{n}$ which inherits many operations from vector spaces. For instance, the direct sum and tensor product of fiber spaces are some of the tools from linear algebra which are naturally adapted to yield operations on vector bundles. In fact, it is proven that any representation $\rho: G L(n, \mathbb{R}) \rightarrow G L(m, \mathbb{R})$ extends to an operation $\rho(X)$ from $V e c t_{n}(X)$ to $\operatorname{Vect}_{m}(X)$, which is natural in the sense that, for any 
map $f: X \rightarrow Y$, we have $\rho(X) \circ f^{*}=f^{*} \circ \rho(X)$.

It is a natural question to ask whether these functors are homotopy invariant:

Theorem 3.2.1: $\quad$ Given a vector bundle $p: E \rightarrow B$ and homotopic maps $f_{0}, f_{1}: A \rightarrow B$, then the induced bundles $f_{0}^{*}(E)$ and $f_{1}^{*}(E)$ are isomorphic if $A$ is compact Hausdorff.

This result is a consequence of the following:

Lemma 3.2.2: $\quad A$ vector bundle $E \rightarrow X \times[a, b]$ is trivial if its restrictions over $X \times[a, c]$ and $X \times[c, b]$ are both trivial for some $c \in(a, b)$.

Proof of Lemma 3.2.2: First, assume $X$ is connected.

Let these restrictions be $E_{1}=p^{-1}(X \times[a, c])$ and $E_{2}=p^{-1}(X \times[c, b])$ and let $h_{1}: E_{1} \rightarrow X \times$ $[a, c] \times \mathbb{R}^{n}$ and $h_{2}: E_{2} \rightarrow X \times[c, b] \times \mathbb{R}^{n}$. These isomorhisms may not agree on $p^{-1}(X \times\{c\})$, but we can make them agree if we replace $h_{2}$ by its composition with the isomorphism $X \times[c, b] \times \mathbb{R}^{n} \rightarrow$ $X \times[c, b] \times \mathbb{R}^{n}$ which on each slice $X \times\{t\} \times \mathbb{R}^{n}$ is given by $h_{1} h_{2}^{-1}: X \times\{c\} \times \mathbb{R}^{n} \rightarrow X \times\{c\} \times \mathbb{R}^{n}$. Since, from such replacement we have defined two trivializations which agree on $E_{1} \cap E_{2}$, they define a trivialization on $E$.

If $E$ is not connected, we apply the procedure above to each connected component.

Lemma 3.2.3: $\quad$ Let $X$ be a compact Hausdorff space. For a vector bundle $p: E \rightarrow X \times I$, there exists an open cover $\left\{U_{\alpha}\right\}$ of $X$ so that each restriction $p^{-1}\left(U_{\alpha} \times I\right) \rightarrow U_{\alpha} \times I$ is trivial.

Proof of Lemma 3.2.3: For each $(x, t) \in X \times I$ we find a collection of open neighbourhoods $U_{x, 1}, \ldots, U_{x, k}$ in $X$ and a partition $0=t_{0}<t_{1}<\cdots<t_{k}=1$, such that the bundle $E$ is trivial over $U_{x, i} \times\left[t_{i-1}, t_{i}\right]$, using compactness of $I$. Using Lemma 3.2 .2 , the bundle is trivial over $U_{\alpha} \times I$ where $U_{\alpha}=U_{x, 1} \cap \cdots \cap U_{x, k}$.

Proposition 3.2.4: $\quad$ The restrictions of a vector bundle $E \rightarrow X \times I$ over $X \times\{0\}$ and $X \times\{1\}$ are isomorphic if $X$ is compact Hausdorff.

Proof of Proposition 3.2.4: From Lemma 3.2.3, we find an open cover $\left\{U_{\alpha}\right\}$ of $X$ so that $E$ is trivial over each $U_{\alpha} \times I$. Since $X$ is compact, we assume that such collection is finite $U_{1}, \ldots, U_{m}$, and there is a partition of unity by functions $\varphi_{i}$ whose support is contained in $U_{i}$. For each $i$, let $\psi_{i}=\varphi_{0}+\cdots+\varphi_{i}$ and let $X_{i}$ be the graph of $\psi_{i}$, that is, the subspace of $X \times I$ formed by the points $\left(x, \phi_{i}(x)\right)$, and let $E_{i} \rightarrow X_{i}$ be the restriction of the bundle $E$. Since $E$ is trivial over $U_{i} \times I$, the natural projection homomorphism $X_{i} \rightarrow X_{i-1}$ lifts to a homomorphism $h_{i}: E_{i} \rightarrow E_{i-1}$ which is the identity outside $p^{-1}\left(U_{i} \times I\right)$ and which takes each fiber of $E_{i}$ isomorphically onto the corresponding fiber of $E_{i-1}$. Explicitly, on points in $p^{-1}\left(U_{i} \times I\right)=U_{i} \times I \times \mathbb{R}^{n}$, we let $h_{i}\left(x, \psi_{i}(x), v\right)=\left(x, \psi_{i-1}(x), v\right)$. The composition $h=h_{1} h_{2} \ldots h_{m}$ is then an isomorphism from the restriction of $E$ over $X \times\{1\}$ to the restriction of $E$ over $X \times\{0\}$.

We now move to the proof of the theorem:

Proof of Theorem 3.2.1: Let $F: A \times I \rightarrow B$ be a homotopy from $f_{0}$ to $f_{1}$. The restriction of $F^{*}(E)$ over $A \times\{0\}$ and $A \times\{1\}$ are then $f_{0}^{*}(E)$ and $f_{1}^{*}(E)$ respectively. So, from proposition 
3.2.4, the result follows.

Corollary 3.2.5: $\quad$ A homotopy equivalence $f: A \rightarrow B$ of compact Hausdorff spaces induces a bijection $f^{*}: \operatorname{Vect}_{n}(B) \rightarrow \operatorname{Vect}_{n}(A)$. In particular, every vector bundle over a contractible compact Hausdorff base is trivial.

A natural question which arises from the previous section is if such homotopy-invariant functors are representable. Indeed, we have:

Theorem 3.2.6: $\quad$ The functors $V$ ect ${ }_{n}$ from compact hausdorff spaces to pointed sets are represented by the infinite Grassmanian $G_{n}$. That is, $\operatorname{Vect}_{n}(X)=\left[X, G_{n}\right]$, where $G_{n}$ is the direct limit of the spaces

$$
\frac{O(n+k)}{O(n) \times O(k)} \subset \frac{O(n+k+1)}{O(n) \times O(k+1)} \subset \ldots \quad k=1,2, \ldots
$$

In particular, on CW-complexes of dimension less then $N$, the functor $V e c t_{n}$ is represented by the finite Grassmanians $G_{n, m}=\frac{O(n+m)}{O(n) \times O(m)}$, for $m \gg N$, which is thought of as the space of all $m$-dimensional subspaces in an euclidean $(n+m)$-euclidean space $V$.

The idea behind the relation between such functors is the following: consider the trivial bundle $\tilde{V}=V \times G_{n, m}$ over $G_{n, m}$ and let $S$ be the subset of $\tilde{V}$ consisting of all pairs $v \in V, A \in G_{n, m}$ with $v \in A$. The projection $(v, A) \rightarrow A$ defines $S$ as a vector bundle over $G_{n, m}$, which is called the universal sub-bundle of $\tilde{V}$. Similarly, the subset $Q=\{(v, A): v$ belongs to the orthogonal complement of $A\}$ defines the universal quotient bundle over $G_{n, m}$. Clearly, $\tilde{V}=S \oplus Q$.

Now, if $E$ is an $n$-dimensional fiber bundle over $X$, we claim that, for $m$ large enough (with respect to the dimension of $X$ ), there is a map $f: X \rightarrow G_{n, m}$, such that $f^{*} Q=E$. Once this fact is established, the assignment $\left[X, G_{n, m}\right] \rightarrow \operatorname{Vect}_{n}(X)$ which sends $f: X \rightarrow G_{n, m}$ into $f^{*} Q$ is clearly one-to-one and onto when $m$ is large enough.

This result is proved in detail on Chapter 1 of [Hat03], using the geometrical idea of clutching functions.

We see that these Grassmanian spaces are closely related to the spaces $B U$ and $B O$ : from the exact sequence of the fibering $O(n) \rightarrow \frac{O(n+m)}{O(m)} \rightarrow \frac{O(n+m)}{O(m) \times O(n)}$, we see that $\pi_{k}\left(O_{n}\right) \simeq$ $\pi_{k+1}\left(G_{n, m}\right)$, if $m \gg n$. Then if $n \gg k$, we have

$$
\begin{array}{rlrl}
\operatorname{Vect}_{n}\left(S^{k+1}\right) & =\left[S^{k+1}, G_{n, m}\right]=\left[p t, \Omega^{k} G_{n, m}\right] & \\
& =\pi_{k+1}\left(G_{n, m}\right)=\pi_{k}(O(n)) & & \text { if } n \gg k
\end{array}
$$

Thus we may interpret Bott's periodicity theorem as evaluating the functor $V e c t_{n}\left(S^{k+1}\right)$ for $n$ large enough compared to $k$.

Although these functors $V e c t_{n}$ have interesting properties which come from operations on vector spaces, we would like to produce functors on $\oplus$ defined a group operation. What is missing on $V e c t_{n}$ is the existence of inverse elements. 
In the category of compact spaces, we have numerous canonical ways of completing such objects to groups and we next define two of them:

To facilitate notation, we denote by $\varepsilon^{n}$ the $n$-trivial bundle over $X$. We define two equivalence relations on the vector bundles over $X$ : we define the relation $\approx_{S}$, by setting $E_{1} \approx_{S} E_{2}$ if $E_{1} \oplus \varepsilon^{n} \simeq E_{2} \oplus \varepsilon^{n}$ for some $n$. We also define the relation $\sim$, by setting $E_{1} \sim E_{2}$, if $E_{1} \oplus \varepsilon^{m} \simeq$ $E_{2} \oplus \varepsilon^{n}$ for some $m, n$.

Theorem 3.2.7: $\quad$ The set of $\sim$-equivalence classes of vector bundles over $X$ is represented by the space BO. In other words, the space of $\sim$-equivalence classes of vector bundles over $X$ is given by $\widetilde{K O}(X)$.

This is a beautiful result which gives us a connection between the functor classified by $B O$, which we defined on the last section, and this functor created from equivalence classes on vector bundles. A proof of this result is seen in [Hat03]. Also, a first "virtue" of this geometric construction of $\widetilde{K O}$ is that it can be easily seen to carry a natural abelian group-structure over compact Hausdorff spaces. This is due to:

Proposition 3.2.8: If $X$ is compact Hausdorff, then the set of $\sim$-equivalence classes of vector bundles over $X$ forms an abelian group with respect to $\oplus$.

The problem here is to assure that every element has an inverse, which is a consequence of:

Proposition 3.2.9: For each vector bundle $E \rightarrow B$ with $B$ connected compact Hausdorff, there exists a vector bundle $E^{\prime} \rightarrow B$ such that $E \oplus E^{\prime}$ is a trivial bundle.

To motivate our proof of this result, we recall that, if the result holds, then $E$ is a sub-bundle of a trivial bundle $B \times \mathbb{R}^{N}$. Composing the inclusion of $E$ into this product with the projection onto $\mathbb{R}^{N}$ yields a map $E \rightarrow \mathbb{R}^{N}$ that is a linear injection on each fiber. Our logic is to make the inverse path: to construct a map $E \rightarrow \mathbb{R}^{N}$ that is a linear injection on each fiber and then show that this gives an embedding of $E$ in $B \times \mathbb{R}^{N}$ as a direct summand.

Proof of Proposition 3.2.9: Each point $x \in B$ has a neighbourhood $U_{x}$ over which $E$ is trivial. By Usysohn's Lemma, there is a map $\varphi_{x}: B \rightarrow[0,1]$ that is 0 outside $U_{x}$ and nonzero at $x$. Letting $x$ vary, the sets $\varphi_{x}^{-1}(0,1]$ form an open cover of $B$. By compactedness, this has a finite subcover. Let the corresponding $U_{x}$ 's and $\varphi_{x}$ 's be relabeled $U_{i}$ and $\varphi_{i}$. Define $g_{i}: E \rightarrow \mathbb{R}^{n}$ by $g_{i}(v)=\varphi_{i}(p(v))\left(\pi_{i} h_{i}(v)\right)$, where $p$ is the projection $E \rightarrow B$ and $\pi_{i} h_{i}$ is the composition of a local trivialization $h_{i}: p^{-1}\left(U_{i}\right) \rightarrow U_{i} \times \mathbb{R}^{n}$, with the projection $\pi_{i}$ to $\mathbb{R}^{n}$. Then $g_{i}$ is a linear injection on each fiber over $\varphi_{i}^{-1}(0,1]$, so if we make the various $g_{i}$ 's the coordinates of a map $g: E \rightarrow \mathbb{R}^{N}$, with $\mathbb{R}^{N}$ a product of copies of $\mathbb{R}^{n}$, then $g$ is a linear injection on each fiber.

The map $g$ is the second coordinate of a map $f: E \rightarrow B \times \mathbb{R}^{n}$ with first coordinate $p$. The image of $f$ is a subbundle of the product $B \times \mathbb{R}^{N}$, since the projection of $\mathbb{R}^{N}$ into the $i$-th $\mathbb{R}^{n}$ factor gives the second coordinate of a local trivialization over $\varphi_{i}^{-1}(0,1]$. Thus we have $E$ isomorphic to a subbundle $E_{*}$ of $B \times \mathbb{R}^{N}$.

Let $E_{*}^{\perp}$ be the subspace of $B \times \mathbb{R}^{N}$, which in each fiber consists of all vectors orthogonal to vectors in $E_{*}$. Then it is simple to show that the projection $E_{*}^{\perp} \rightarrow B$ is a vector bundle and 
clearly $E \oplus E_{*}^{\perp}$ is isomorphic to $B \times \mathbb{R}^{N}$ via the map $(v, w) \rightarrow v+w$.

Proof of Proposition 3.2.8: If all fibers of $E$ have the same dimension, we simply apply Proposition 3.2.9. In the general case, let $X_{i}=\left\{x \in X \mid \operatorname{dim} \pi^{-1}(x)=i\right\}$. These $X_{i}$ are disjoint open sets in $X$, and therefore are in finite number by compactness. By adding to $E$ a bundle which over to each $X_{i}$ is a trivial bundle of suitable dimension, we produce a bundle whose fibers all have the same dimension and apply Proposition 3.2 .9 .

The connection between the geometrical and representable aspects of this functor yields many interesting results. For instance, from the relation $\widetilde{K O}\left(S^{1}\right)=\mathbb{Z}_{2}$, we think of the vector bundles on $S^{1}$ as having only one non-trivial element of order 2 (regarding the relation $\sim$ ). We see that the Möbius bundle is a representative of this class, and it can be seen to have order two by embedding two Möbius bands in a solid torus so that they intersect orthogonally along the common core circle of both bands. This provides a visualisation to the fact that the sum of two Möbius bundles is the product $S^{1} \times \mathbb{R}^{2}$.
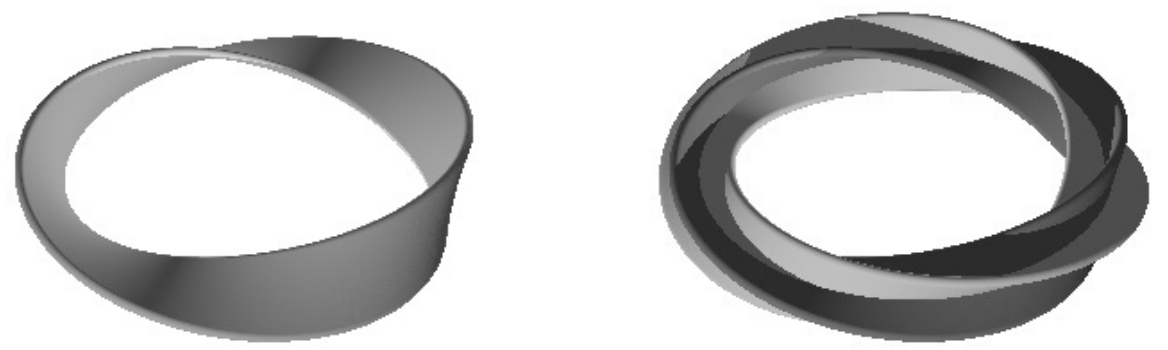

Following a different path, we look at the set of $\approx_{S^{-}}$equivalence classes of vector bundles. This is not a group yet because if $E$ is an invertible vector bundle over $\oplus$ on this classes, then there is a bundle $E^{\prime}$ such that $E \oplus E^{\prime} \approx_{S} \varepsilon^{0}$, which means that $E \oplus E^{\prime} \oplus \varepsilon^{n} \simeq \varepsilon^{n}$, for some $n$. Since this only happens if the dimension of $E$ is zero, the only invertible element is $\varepsilon^{0}$. However, we have a cancellation property: If $E_{1} \oplus E_{2} \approx_{S} E_{1} \oplus E_{3}$, we add to both sides a bundle $E_{1}^{\prime}$, such that $E_{1}^{\prime} \oplus E_{1} \simeq \varepsilon^{n}$, and therefore, we get that $E_{2} \approx_{S} E_{3}$.

Then we construct a group from this $\approx_{S}$-classes using the same strategy one uses to build the integers from the natural numbers: we form the group of the formal differences $E-E^{\prime}$ of vector bundles $E$ and $E^{\prime}$ over $X$, with the equivalence relation $E_{1}-E_{1}^{\prime}=E_{2}-E_{2}^{\prime}$ if and only if $E_{1} \oplus E_{2}^{\prime} \approx_{S} E_{2} \oplus E_{1}^{\prime}$. We see that this is a group and that each element can be represented as a difference $E-\varepsilon^{n}$. This group will be momentarily denoted $\underline{K O}(X)$ and we show that it coincides with the functor $K O$.

We see there is a natural homomorphism $\underline{K O}(X) \rightarrow \widetilde{K O}(X)$ sending $E-\varepsilon^{n}$ to the $\sim$-equivalence class of $E$. This is clearly surjective and its kernel is the subgroup $\left\{\varepsilon^{m}-\varepsilon^{n}\right\}$, which is isomorphic to $\mathbb{Z}$.

In fact, the restriction of vector bundles to a basepoint $x_{0} \in X$ defines a homomorphism $\underline{K O}(X) \rightarrow K O\left(x_{0}\right) \simeq \mathbb{Z}$ which restricts to an isomorphism on the subgroup $\left\{\varepsilon^{m}-\varepsilon^{n}\right\}$. This, which can be thought as a map associating each bundle to its dimension on the connected component of $x_{0}$, gives us a splitting $\underline{K O}(X) \simeq \widetilde{K O}(X) \oplus \mathbb{Z}$ depending on the choice of $x_{0}$. From 
this relation, we get that $\underline{K O}$ is represented by $B O \times \mathbb{Z}$ as was defined in the previous section, and is the functor $K O$.

Besides the additive structure on $K O(X)$, the operations on vector bundles can be used to define even more structure on this space. For instance, we use a tensor product to define a multiplication: we define the product of classes represented by $E_{1}$ and $E_{2}$ as the class represented by $E_{1} \otimes E_{2}$. We see that, for arbitrary elements of $K(X)$, we have the multiplication defined by the following relation

$$
\left(E_{1}-E_{1}^{\prime}\right)\left(E_{2}-E_{2}^{\prime}\right)=\left(E_{1} \otimes E_{2}+E_{1}^{\prime} \otimes E_{2}^{\prime}\right)-\left(E_{1}^{\prime} \otimes E_{2}+E_{1} \otimes E_{2}^{\prime}\right)
$$

It is simple to verify that this operation is well-defined and makes $K O(X)$ into a commutative ring with identity $\varepsilon^{1}$, the trivial line bundle.

Also, if we choose a basepoint $x_{0} \in X$, the map $K O(X) \rightarrow K O\left(x_{0}\right)$ obtained by restricting vector bundles to their fibers over $x_{0}$ is a ring homomorphism. The identification of $\widetilde{K O}(X)$ with the kernel of this map also provides a ring structure on it, although not necessarily with identity.

Then we have two interpretations to a theory, which yield extremely interesting results: from the geometric approach, we see that these functors have values in rings carrying various operations deduced from linear algebra; while from the representable viewpoint, we get a functor which leads us to an 8-fold periodic cohomology theory whose values on the spheres are given by Bott's Periodicity Theorem, and which yields the relation

$$
K O\left(S^{n+8} X\right) \simeq K O\left(S^{n} X\right)
$$

Bott's Periodicity Theorem can also be given a geometric interpretation on such a context, which yields the Functorial Form of the Periodicity Theorem. We now discuss it briefly.

From the properties discussed in the last section and the relation $S^{n} X=\frac{S^{n} \times X}{S^{n} \vee X}$, we get

$$
\widetilde{K O}\left(S^{n} \vee X\right) \oplus K O\left(S^{n} X\right)=K O\left(S^{n} \times X\right)
$$

On the other hand, from the geometric $K O$, we have a natural map $K O(X) \otimes K O(Y) \rightarrow$ $K O(X \times Y)$, sending $E \otimes F$ to $\left[p_{1}^{*} E p_{2}^{*} F\right]$ where $p_{1}, p_{2}$ are the natural projections.

Then Bott's result can be reformulated in:

Theorem 3.2.10: $\quad$ (The Real Periodicity Theorem) The tensor product of bundles induces an isomorphism

$$
K O(x) \otimes K O\left(S^{8}\right) \simeq K O\left(X \times S^{8}\right)
$$

Applying the constructions above to complex vector bundles yields the geometrical interpretation behind the functors $\tilde{K}$ and $K$, which endows them with natural ring structures and operations derived from linear algebra. Also, Bott's Periodicity Theorem for the group $U$ can be reformulated 
as follows:

Theorem 3.2.11: (The Complex Periodicity Theorem) The tensor product of bundles induces an isomorphism

$$
K(x) \otimes K\left(S^{2}\right) \simeq K\left(X \times S^{2}\right)
$$

Sometime after Bott's article [Bot59], many other proofs of the Periodicity Theorem for the Classical Groups were formulated. One such proof was developed by Atiyah and Bott, using the above interpretation of the result and based on properties of vector bundles. This proof is seen in Chapter II of AA67.

We close this chapter by stating two deep and important results which were obtained using K-Theory (the proofs can be found in Section 2.3 of [Hat03]):

Theorem 3.2.12: $\quad \mathbb{R}^{n}$ is a division algebra if only if $n=1,2,4$ and 8 .

Theorem 3.2.13: $\quad$ A sphere $S^{n}$ is said to be parallelizable if there exists $n$ tangent vector fields to $S^{n}$ which are linearly independent at each point, or in other words, if the tangent bundle to $S^{n}$ is trivial. The only parallelizable spheres are $S^{0}, S^{1}, S^{3}$ and $S^{7}$.

These are only a few examples of the applications of this beautiful theory which combines geometrical and representable aspects and which is fairly computable due to Bott's Periodicity Theorem. 


\section{Bibliography}

[AA67] M.F. Atiyah and D.W. Anderson. K-theory, volume 216. WA Benjamin New York, 1967.

[AB95] D. M Austin and P.J. Braam. Morse-bott theory and equivariant cohomology. The Floer memorial volume, 133:123-183, 1995.

[Ada95] J.F. Adams. Stable homotopy and generalised homology. University of Chicago press, 1995.

[Bas] S. Basu. What is an eilenberg-maclane space? Disponível em http://www.math. binghamton.edu/somnath/Notes/GSS4.pdf. Last visited in 20/06/2016.

[BH13] A. Banyaga and D. Hurtubise. Lectures on Morse homology, volume 29. Springer Science \& Business Media, 2013.

[Bot54] R. Bott. Nondegenerate critical manifolds. Annals of Mathematics, pages 248-261, 1954.

[Bot59] R. Bott. The stable homotopy of the classical groups. Annals of Mathematics, pages 313-337, 1959.

[Bot69] R. Bott. Lectures on k(x). 1969.

[Bot70] R. Bott. The periodicity theorem for the classical groups and some of its applications. Advances in Mathematics, 4(3):353-411, 1970.

[Bro62] E.H. Brown. Cohomology theories. Annals of Mathematics, pages 467-484, 1962.

[Bro06] D. Brown. Serre Spectral Sequences and Applications, Lecture Notes: Algebraic Topology, 2006. URL: http://www . mathcs . emory . edu/ dzb/math/papers/sss.pdf. Last visited on 2017/07/14.

[BS58] R. Bott and H. Samelson. Applications of the theory of morse to symmetric spaces. American Journal of Mathematics, 80(4):964-1029, 1958.

[BT82] R. Bott and L. Tu. Differential Forms in Algebraic Topology. Graduate Texts in Mathematics No. 82, Springer-Verlag, 1982.

[Cho06] T.Y. Chow. You could have invented spectral sequences. Notices of the AMS, 53:1519, 2006. 
[Die09] J. Dieudonné. A history of algebraic and differential topology, 1900-1960. Springer Science \& Business Media, 2009.

[DK01] J.F. Davis and P. Kirk. Lecture Notes in Algebraic Topology. Graduate Studies in Mathematics, American Mathematical Society, 2001.

[Eil49] S. Eilenberg. On the problems of topology. Annals of Mathematics, pages 247-260, 1949.

[ES45] S. Eilenberg and N.E. Steenrod. Axiomatic approach to homology theory. Proceedings of the National Academy of Sciences, 31(4):117-120, 1945.

[GM81] P.A. Griffiths and J.W. Morgan. Rational Homotopy Theory and Differential Forms. Progress in Mathematics, 16. Birkhäuser, 1981.

[Gro] M. Groth. Section 11: Killing homotopy groups: Postnikov and whitehead towers. Disponível em http://www.math.ru.nl/〜mgroth/teaching/htpy13/ Section11.pdf. Last visited in 20/06/2016.

[Hat02] A. Hatcher. Algebraic Topology. Cambridge University Press, 2002.

[Hat03] A. Hatcher. Vector bundles and k-theory. Im Internet unter http://www. math. cornell. edu/ hatcher, 2003. Last visited on 2017/07/10".

[Hat04] A. Hatcher. Spectral sequences in algebraic topology. Unpublished book project, available on http://www. math. cornell. edu/hatcher/SSAT/SSATpage. html, 22, 2004.

[Hir12] M.W. Hirsch. Differential topology, volume 33. Springer Science \& Business Media, 2012 .

[Hur52] W. Hurewicz. Homology and homotopy theory. In Proceedings of the International Mathematical Congress Held..., page 344. University of Toronto Press, 1952.

[Jam99] I.M. James. History of topology. Elsevier, Amsterdam, 1999.

[Mat02] Y. Matsumoto. An introduction to Morse theory, volume 208. American Mathematical Soc., 2002.

[Mau96] C.R.F. Maunder. Algebraic topology. Courier Corporation, 1996.

[May99] J.P. May. A concise course in algebraic topology. University of Chicago press, 1999.

[McC99] J. McCleary. A history of spectral sequences: Origins to 1953. In I.M. James, editor, History of Topology, chapter 23, pages 631-664. Elsevier, Amsterdam, 1999.

[McC01] J. McCleary. A user's guide to spectral sequences. Number 58. Cambridge University Press, 2001.

[Mil56] J. Milnor. On manifolds homeomorphic to the 7-sphere. Annals of Mathematics, pages 399-405, 1956.

[Mil63] J. Milnor. Morse Theory. Princeton University Press, 1963.

[Mil15] J. Milnor. Lectures on the h-cobordism theorem. Princeton University Press, 2015. 
[MT68] R. E Mosher and M. C. Tangora. Cohomology operations and applications in homotopy theory. Courier Corporation, 1968.

[MW64] J. Milnor and A. Wallace. Differential topology. Lectures on modern mathematics, 2:165-183, 1964.

[Nic11] L. Nicolaescu. An invitation to Morse theory. Springer Science \& Business Media, 2011.

[Nov12] S.P. Novikov. Topological Library: Spectral sequences in topology. World Scientific, 2012.

[Pal69] R.S. Palais. The morse lemma for banach spaces. Bulletin of the American Mathematical Society, 75(5):968-971, 1969.

$\left[\mathrm{SBM}^{+} 12\right]$ J.P. Serre, A. Borel, J. Milnor, J.F. Adams, M.F. Atiyah, F. Hirzebruch, S.P. Novikov, and I.A. Taimanov. Homotopy groups and classes of abelian groups. Topological Library: Part 3: Spectral Sequences in Topology, 50:105, 2012.

[Ser53] J.P. Serre. Groupes d'homotopie et classes de groupes abéliens. Annals of Mathematics, pages 258-294, 1953.

[Ser12] J.P. Serre. Singular homology of fiber spaces'. Topological Library: Part 3: Spectral Sequences in Topology, 50:1, 2012.

[Swi75] R.M. Switzer. Algebraic topology: homotopy and homology, volume 212. Springer, 1975.

[Whi78] G. Whitehead. Elements of Homotopy Theory. Graduate Texts in Mathematics No. 61, Springer-Verlag, 1978.

[Whi12] H. Whitney. Geometric integration theory. Courier Corporation, 2012. 